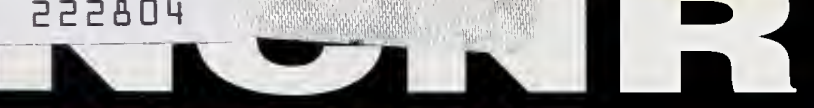

NIST GENTER FOR NEUTRON RESEARCH ACCOMPLISHMENTS AND OPPORTUNITIES

\title{
NGT
}

National Institute of Standards and Technology Technology Administration U.S. Department of Commerce February 2001 NIST SP 962
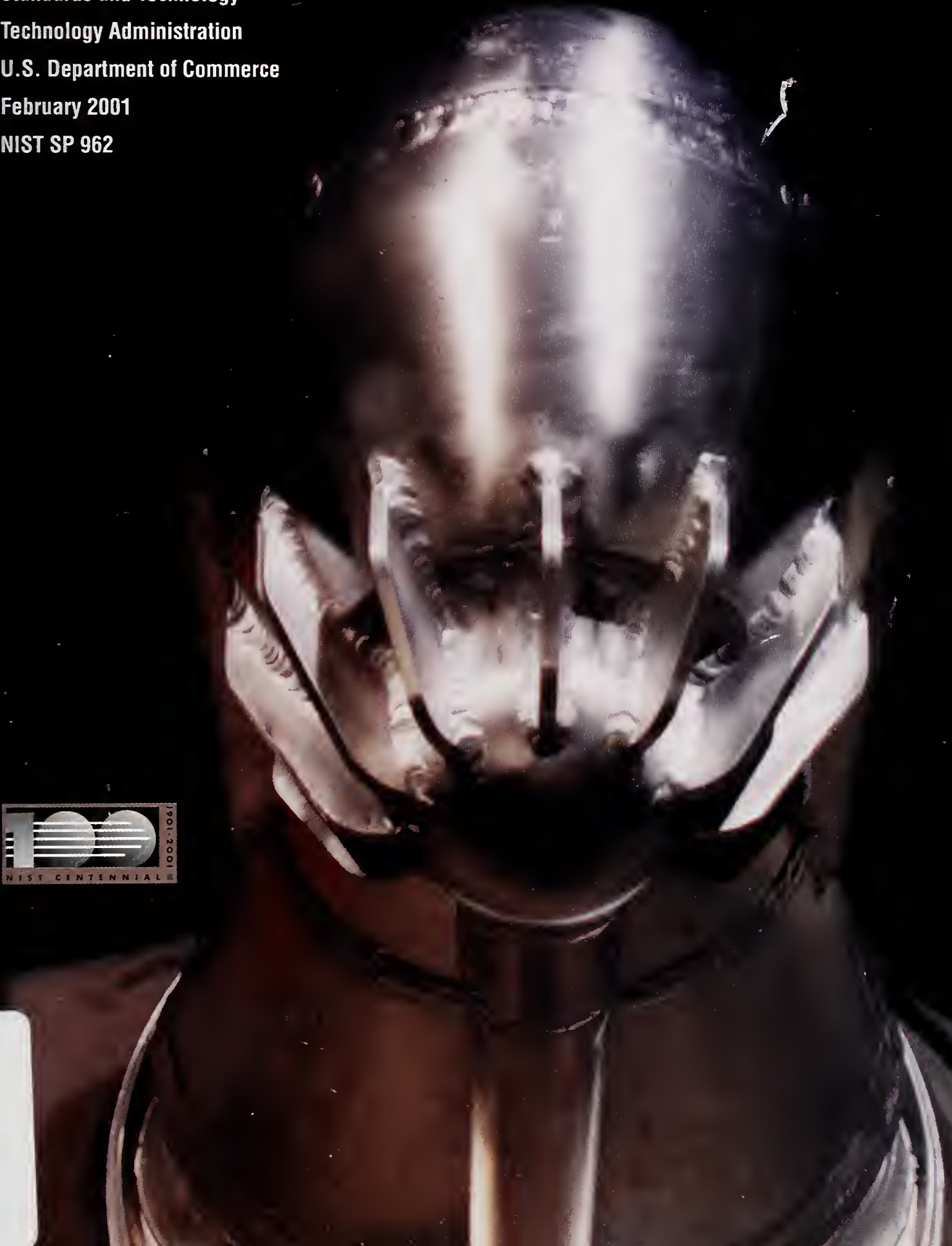


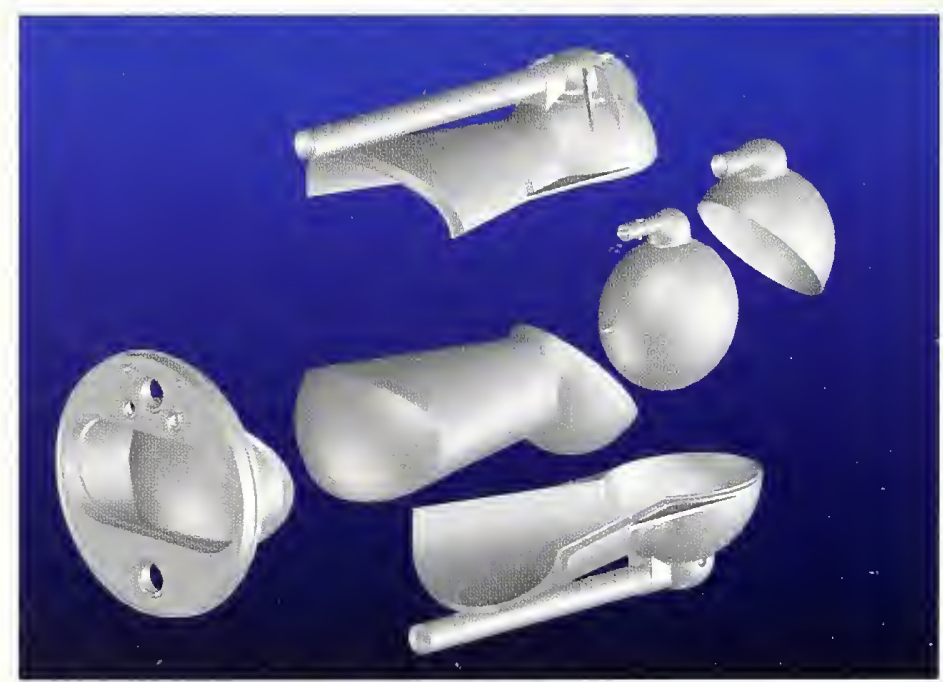

\section{ON THE COVER}

The inset shows an exploded view of welded aluminum components of the second-generation liquid hydrogen cold source to be installed during 2001. The cold source, which slows down thermal neutrons, is the round object located right-middle. Surrounding it is a vacuum jacket located just to the right and left of the cold source. The outer layer, exploded top and bottom and to the left, is the vacuum shroud that is also shown standing vertically on the front cover. Cold neutrons emerge from the cap shown at the left into 8 guides carrying them to beam instruments. (See layout on p. 2.) 


\section{NCNR 2000}

\section{NIST}

CENTER FOR

NEUTRON

RESEARCH

ACCOMPLISHMENTS

\section{AND}

\section{OPPORTUNITIES}

\section{NIST Special Publication 962}

J. Michael Rowe, Director

Ronald L. Cappelletti, Editor

Linda K. Clutter, Assistant Editor

February 2001

U.S. DEPARTMENT OF COMMERCE

Donald L. Evans, Secretary

Technology Administration

Karen H. Brown, Acting Under Secretary of Commerce for Technology

National Institute of Standards and Technology

Karen H. Brown, Acting Director

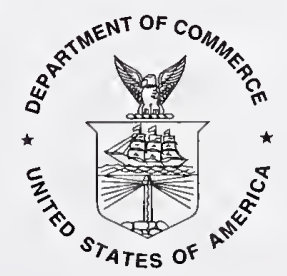




\section{DISCLAIMER}

Certain commercial equipment, instruments, or materials are identified in this report to foster understanding. Such identification does not imply recommendation or endorsement by the National Institute of Standards and Technology, nor does it imply that the materials or equipment identified are necessarily the best available for the purpose.

National Institute of Standards and Technology Special Publication 962

Natl. Inst. Stand. Technol. Spec. Publ. 962, 76 pages (February 2001)

CODEN: NSPUE2

U.S. GOVERNMENT PRINTING OFFICE - WASHINGTON: 2001

For Sale by the Superintendent of Documents, U.S. Government Printing Office

Washington, DC 20402-9325 


\section{CONTENTS}

FOREWORD

INTRODUCTION

NCNR LAYOUT

NCNR IMAGES 2000

RESEARCH HIGHLIGHTS

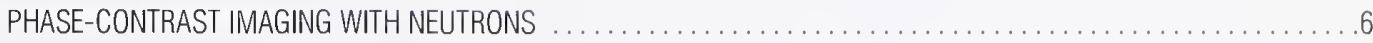

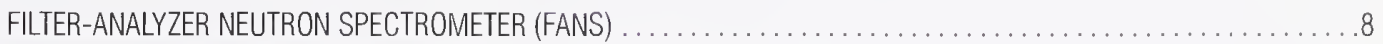

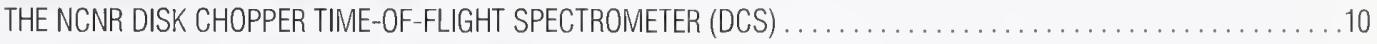

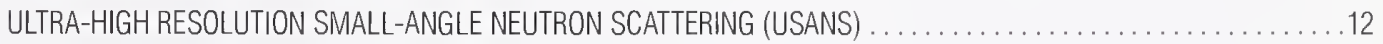

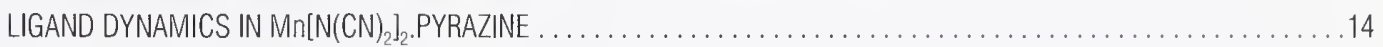

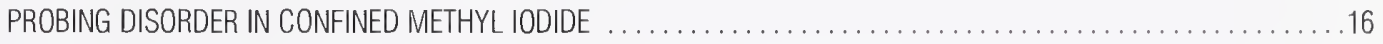

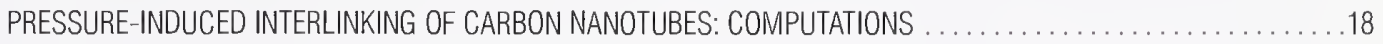

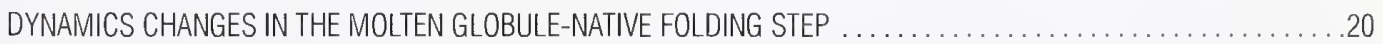

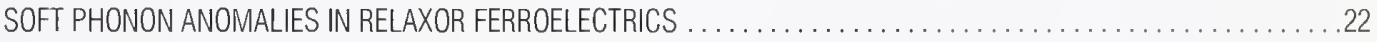

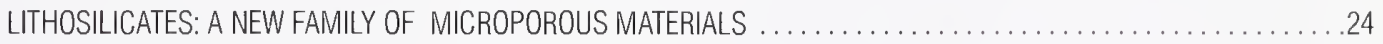

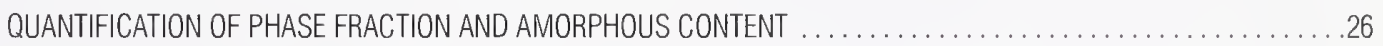

DIFFRACTION ELASTIC CONSTANTS FOR ARBITRARY SPECIMEN AND CRYSTAL SYMMETRIES $\ldots \ldots \ldots \ldots \ldots \ldots$

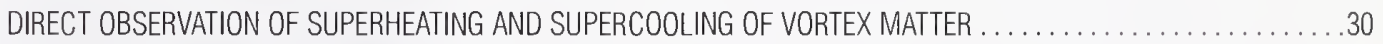

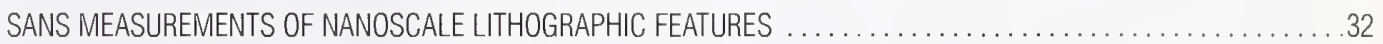

ASYMMETRIC MAGNETIZATION REVERSAL IN EXCHANGE-BIASED HETEROSTRUCTURES $\ldots \ldots \ldots \ldots \ldots \ldots \ldots \ldots \ldots$

THE ROLE OF THE CRP/CAMP PROTEIN COMPLEX IN DNA TRANSCRIPTION $\ldots \ldots \ldots \ldots \ldots \ldots \ldots \ldots \ldots \ldots$

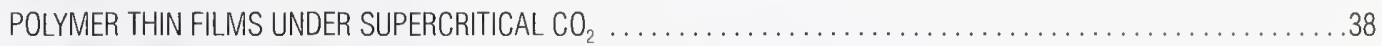

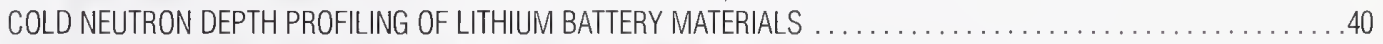

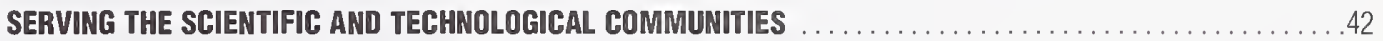

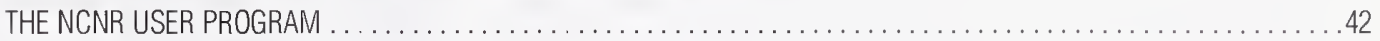

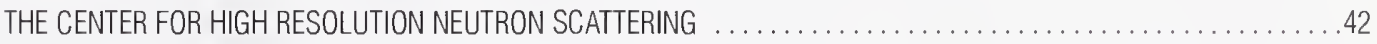

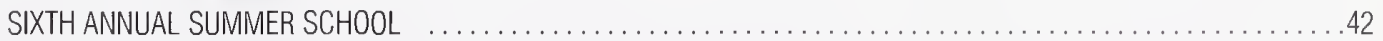

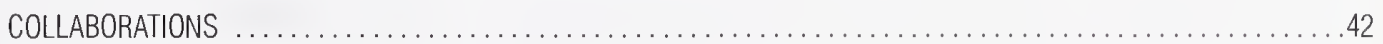

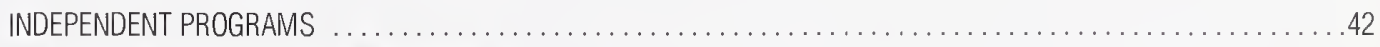

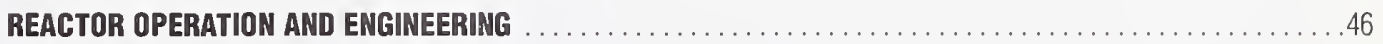

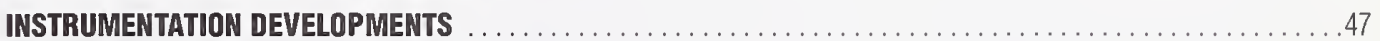

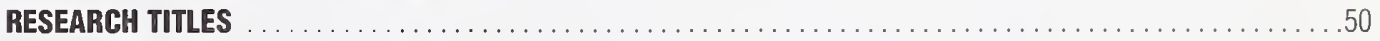

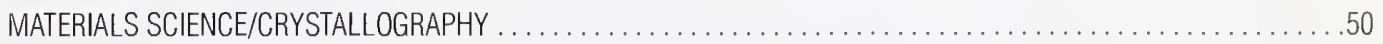

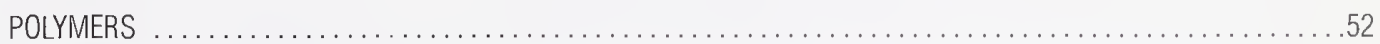

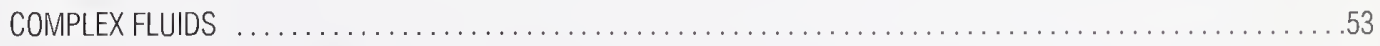

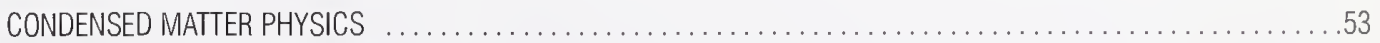

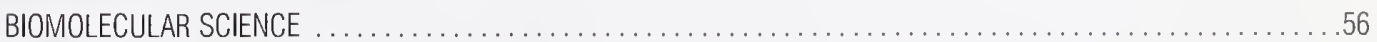

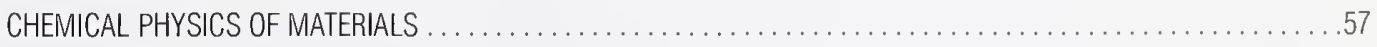

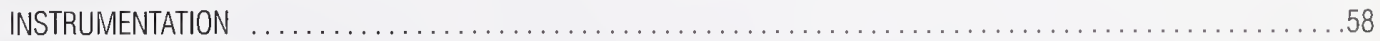

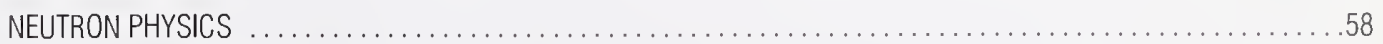

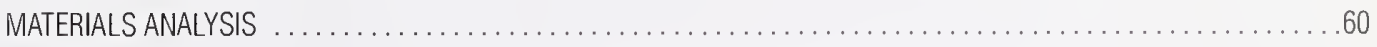

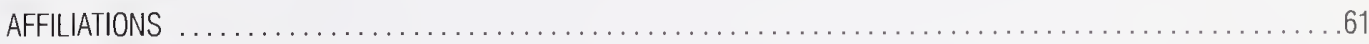

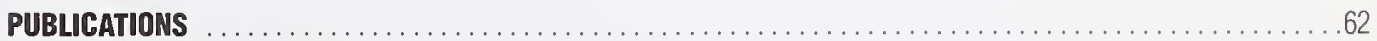

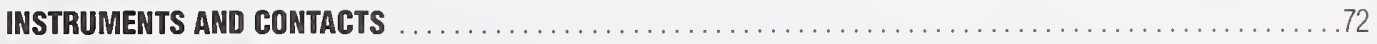

CONTACTS . . . . . . . . . . . . . . . . . . . . . . . . . . . . . . . . . . . . . . . . . . . Inside Back Cover 
O nce again, it is a pleasure to be able to reflect on the accomplishments of the NIST Center for Neutron Research over the past year. The reactor shim control arms were replaced during a planned shutdown early in 2000. As a result, the reactor was scheduled to operate for 212 days during the reporting period, and did operate 198 days, or $93 \%$ of the scheduled time, as a consequence of one unplanned maintenance shutdown. Construction was begun on the new cooling tower which will not only provide needed capability for the next 25 years, but will also reduce the plume visibility during cold weather. The cold source availability for the period was $98 \%$; i.e., the cold source held the reactor from operation 4 days during the year. The second-generation liquid hydrogen cold source passed all required pressure tests, and the final assembly is now being prepared for insertion into the reactor in 2001. Finally, steady progress has been made in preparing for a license renewal application to the Nuclear Regulatory Commission, in order to extend the period of operation beyond 2004.

Three high-resolution inelastic scattering instruments, the High Flux Backscattering Spectrometer, the Disk Chopper time-offlight Spectrometer, and the Neutron Spin Echo spectrometer (highlighted in the 1999 report), are now being offered to users who can tolerate the quirks inherent in getting a new instrument on-line. USANS, the perfect crystal small angle scattering spectrometer (part of the NSF/NIST CHRNS), is installed at the reactor and available for proposals; the first phase of the high intensity Filter Analyzer Neutron Spectrometer is operating with high intensity and good backgrounds; and the design and manufacture of new thermal neutron spectrometers is underway. (USANS, DCS, and FANS are highlighted in this report.) This simultaneous development program has put severe strains on our resources, but we can now look forward to many years of benefit from the results. During the past year, a proposal was made to the National Science Foundation (NSF) to allow joint NIST/NSF operation of the three high-resolution instruments, and to construct a new cold neutron triple axis spectrometer. The final disposition of this application is not yet available, but the external reviews are complete, a site visit has taken place, and the NSF is now considering the appropriate action. If this proposal is funded, then we will be able to operate these new inelastic scattering instruments properly in a full user mode.

Finally, as always, the results are seen in the output of the researchers who use the facility. As has become our practice, we are presenting highlights of this work in the following chapters of this report. I think that all can agree that the results truly speak for themselves.
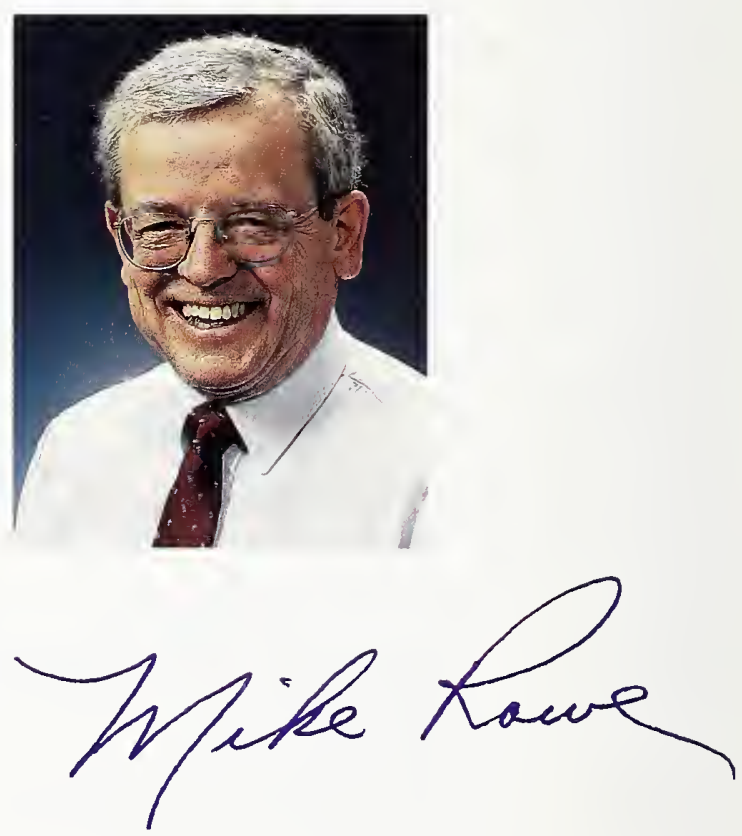


\section{INTRODUCTION TO \\ THE NIST CENTER FOR \\ NEUTRON RESEARCH (NCNR)}

$\mathbf{M}$ odern technological society is dependent upon increasingly sophisticated use of materials, many of whose attributes are dictated by their sub-microscopic structural and dynamical properties. A wide range of scientific techniques, of which the many types of scattering (for example, $\mathrm{x}$-rays, light, electrons, neutrons) are arguably the most important, provide knowledge of these properties. Of these probes, neutrons are perhaps least familiar, but they provide important advantages for many types of measurements.

Neutrons, as prepared for use at modern sources, are moving at speeds comparable to those of atoms moving at room temperature, thus providing the ability to probe dynamical behavior. At the same time, neutrons are well matched to measurements at length scales ranging from the distances between atoms to the size of biological or polymer macromolecules. Neutrons are sensitive to the magnetic properties of atoms and molecules, allowing study of the underlying magnetic properties of materials. They also scatter quite differently from normal hydrogen atoms than they do from heavy hydrogen (deuterium), allowing selective study of individual regions of molecular systems. Finally, neutrons interact only weakly with materials, providing the opportunity to study samples in different environments more easily (at high pressures, in shear, in reaction vessels, etc.), and making them a non-destructive probe. These favorable properties are offset by the relative weakness of the best neutron sources compared to $\mathrm{x}$-ray or electron sources, and by the relatively large facilities required to produce neutrons. As a result, major neutron sources are operated as national user facilities to which researchers come from all over the United States (and abroad) to perform small-scale science using the special measurement capabilities provided.

In addition to scattering measurements, neutrons can be used to probe the atomic composition of materials by means of capture and resultant radioactive decay. The characteristics of the decay act as "fingerprints" for particular atomic nuclei, allowing studies of environmental samples for pollutants (e.g., heavy metals), characterization of Standard Reference Materials, and many other essential measurements. While the scattering and capture users of neutrons are little concerned with understanding the inherent properties of the neutron, there are important areas in physics that can be explored by carefully measuring fundamental neutron behavior. Examples include the lifetime of the free neutron, an important quantity in the theory of astrophysics; the beta decay process of the neutron, the details of which are stringent tests of nuclear theory; and the effects of various external influences such as gravity or magnetic fields on neutrons.

The NCNR utilizes neutrons produced by the 20 MW NIST Research Reactor to provide facilities, including the Nation's only internationally competitive cold neutron facility, for all of the above types of measurements to a national user community. There are approximately 35 stations in the reactor and its associated beams that can provide neutrons for experiments. At the present time 27 of these are in active use, of which 6 provide high neutron flux positions in the reactor for irradiation, and 21 are beam facilities. A schematic layout of the beam facilities and brief descriptions of available instrumentation are given below. More complete descriptions can be found at http://www.ncnr.nist.gov.

These facilities are operated both to serve NIST mission needs and as a national facility, with many different modes of access. Some instrumentation was built years ago, and is not suited to general user access; however, time is available for collaborative research. NIST has recently built new instrumentation (see the highlights in this report on FANS, DCS, and USANS), and reserves $1 / 3$ of available time for mission needs with the balance available to general users. In other cases, instrumentation was built and is operated by Participating Research Teams (PRT); PRT members have access to $75 \%$ of available time, with the balance available to general users. In a special case, NIST and the National Science Foundation established the Center for High Resolution Neutron Scattering at the NCNR, with a $30 \mathrm{~m}$ Small Angle Scattering (SANS) instrument, a cold neutron triple axis spectrometer, and the thermal neutron perfect crystal SANS commissioned this year. For these facilities, most time is available for general users. While most access is for research, whose results are freely available to the general public, proprietary research can be performed under full cost recovery. Each year, approximately 1600 researchers (persons who participated in experiments at the facility, but did not necessarily come here) from all areas of the country, from industry, academe, and government use the facility for measurements not otherwise possible. The research covers a broad spectrum of disciplines, including chemistry, physics, biology, materials science, and engineering. 


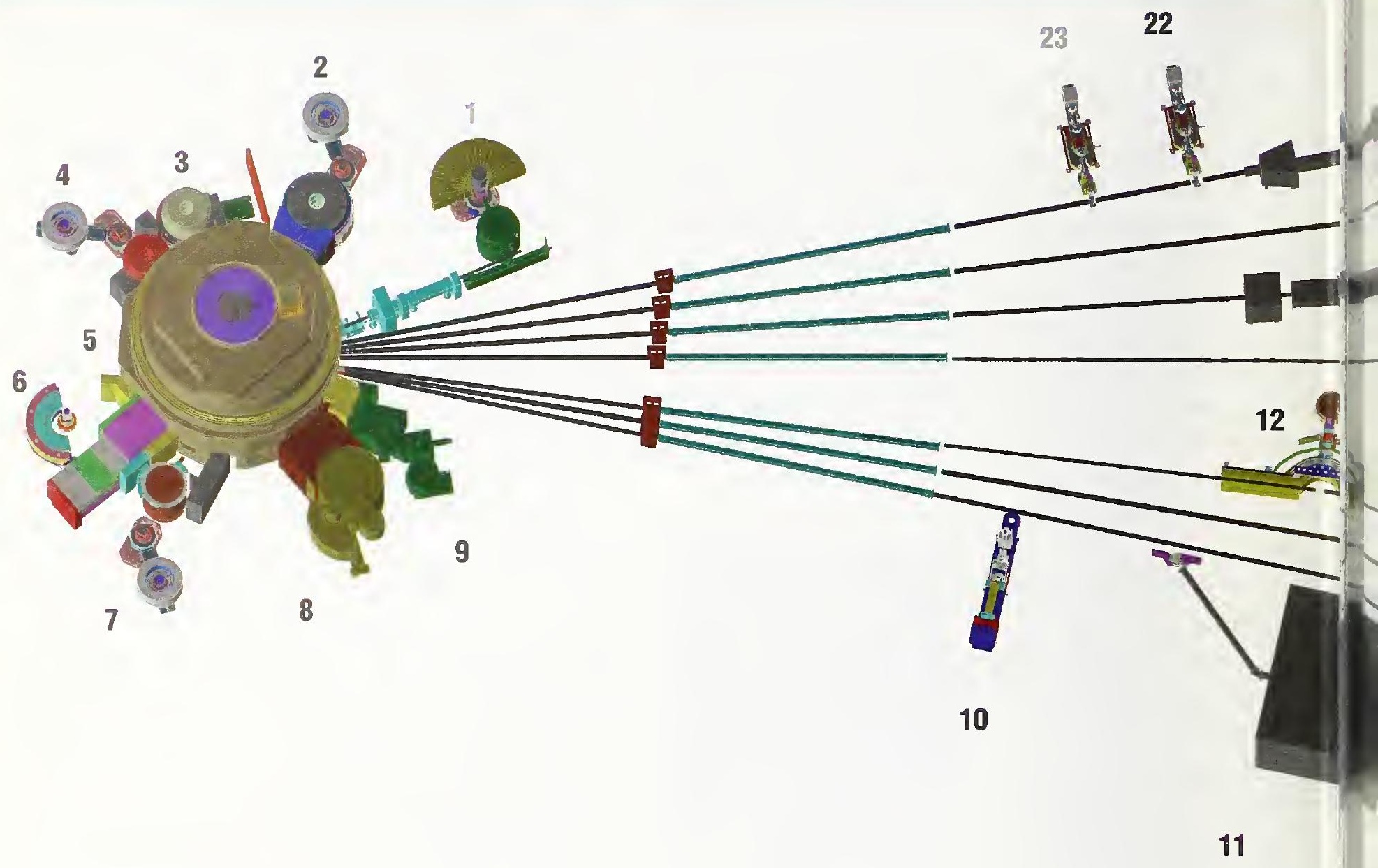

1 A Cold Neutron Depth Profiling instrument (not shown) for quantitative profiling of subsurface impurities currently at this site will be moved to another position. Shown is a proposed Triple Axis Cold Neutron Crystal Spectrometer with double focusing monochromator and multiple crystal analyzer/detectors that can be flexibly configured for several energies simultaneously or for high throughput at one energy.

2 BT-7 Triple Axis Spectrometer with fixed incident energy for measurements of excitations and structure.
3 BT-8 Residual Stress Diffractometer optimized for depth profiling of residual stress in large components.

4 BT-9 Triple Axis Crystal Spectrometer for measurements of excitations and structure.

5 Thermal Column A very wellthermalized beam of neutrons used for radiography, tomography, dosimetry and other experiments.

6 BT-1 Powder Diffractometer Powder diffractometer with 32 detectors; incident wavelengths of $0.208 \mathrm{~nm}, 0.154 \mathrm{~nm}$, and $0.159 \mathrm{~nm}$, with highest resolution of $\delta d / d=8 \times 10^{-4}$.
7 BT-2 Triple Axis Crystal Spectrometer with polarized beam capability for measurement of magnetic dynamics and structure.

\section{BT-4 Filter Analyzer} Spectrometer with cooled Be/Graphite filter analyzer for chemical spectroscopy.

\section{BT-5 Perfect Crystal} Diffractometer SANS small angle neutron scattering instrument for microstructure on the $10^{4} \mathrm{~nm}$ length scale sponsored by the National Science Foundation and NIST, part of the Center for High Resolution Neutron Scattering (CHRNS).
10 NG-7 Horizontal Sample Reflectometer allows reflectivity measurements of free surfaces, liquid vapor interfaces, as well as polymer coatings.

11 Neutron Interferometry and Optics Station with perfect silicon interferometer; vibration isolation system provides exceptional phase stability and fringe visibility.

12 Spin Polarized Triple Axis Spectrometer (SPINS) using cold neutrons with position sensitive detector capability for high resolution studies, part of CHRNS. 


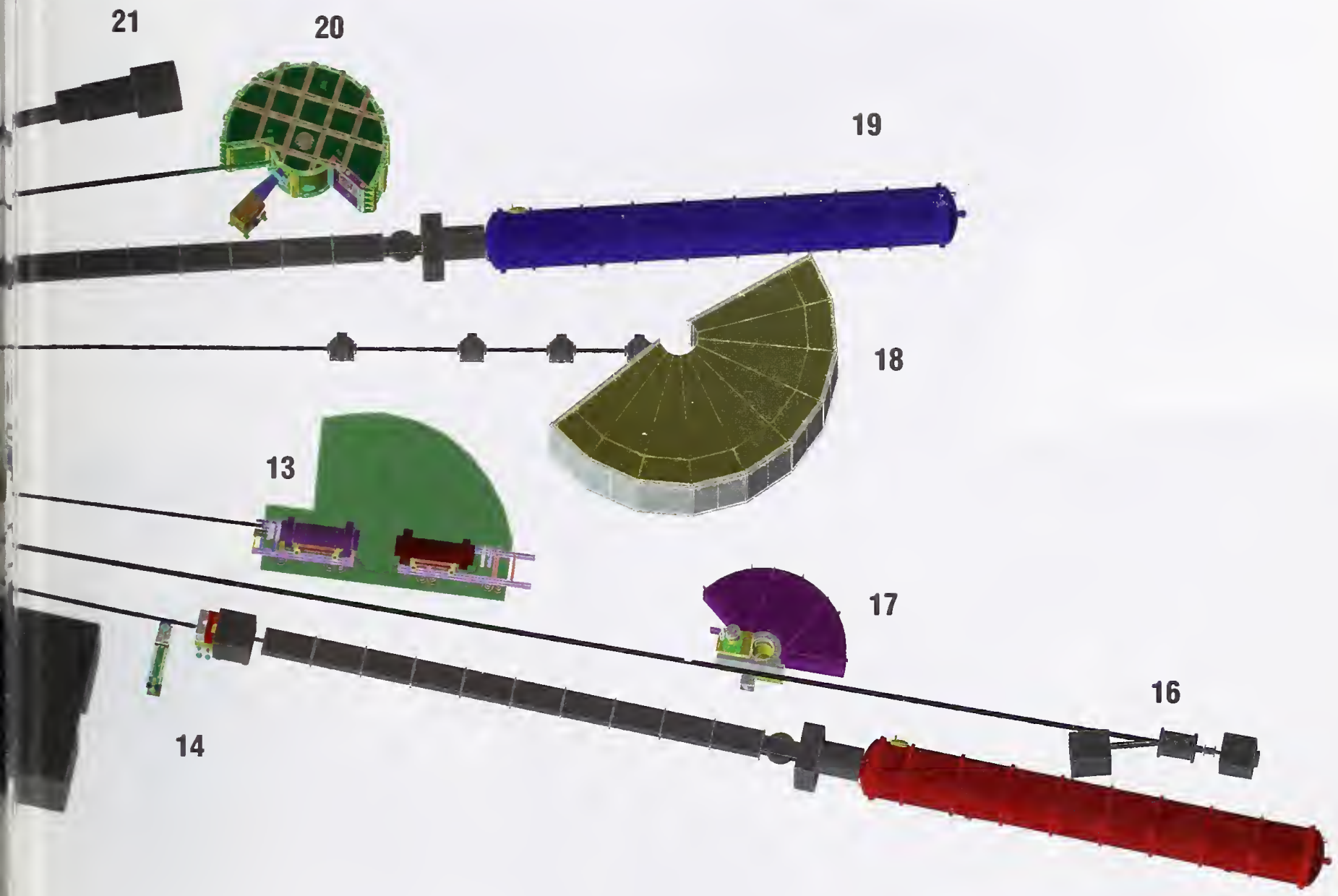

15

13 Spin Echo Spectrometer offering neV energy resolution, based upon Jülich design, sponsored by NIST, Jülich and ExxonMobil.

\section{Prompt Gamma Activation} Analysis cold neutron fluxes allow detection limit for $\mathrm{H}$ of $1 \mu \mathrm{g}$ to $10 \mu \mathrm{g}$. Focused beams are available for profiling.

15 NG-7 30 m SANS for microstructure measurements sponsored by NIST, ExxonMobil, and the University of Minnesota.

16 Neutron Physics Station a cold neutron beam $150 \mathrm{~mm}$ x 60 $\mathrm{mm}$, available for fundamental neutron physics experiments.
17 Fermi Chopper TOF

Spectrometer a hybrid time-offlight spectrometer for inelastic scattering with incident wavelengths between $0.23 \mathrm{~nm}$ and $0.61 \mathrm{~nm}$ chosen by focusing pyrolytic graphite crystals. A simple Fermi chopper pulses the beam.

\section{Disk Chopper TOF}

Spectrometer versatile timeof-flight spectrometer, with beam pulsing and monochromatization effected by 7 disk choppers. Used for studies of dynamics in condensed matter, including macromolecular systems.
19 NG-3 30 m SANS for microstructure measurements sponsored by the National Science Foundation and NIST; part of CHRNS.

20 Backscattering Spectrometer: high intensity inelastic scattering instrument with energy resolution $<1 \mu \mathrm{eV}$, for studies of motion in molecular and biological systems.

$218 \mathrm{~m}$ SANS for polymer characterization, sponsored by NIST Polymers Division.

\section{Vertical Sample}

Reflectometers: instruments for measuring reflectivities down to $10^{-8}$ to determine subsurface structure, with polarization analysis capability. №. 23 is a proposed instru-ment optimized for biological measurements. 
Right: NCNR, cross-country skier's view. Below: Sushil Satija (NCMR) emphasizes a point about reflectometry to participants in the 6th annual summer school on meutron scattering held June 5-9, 2000.

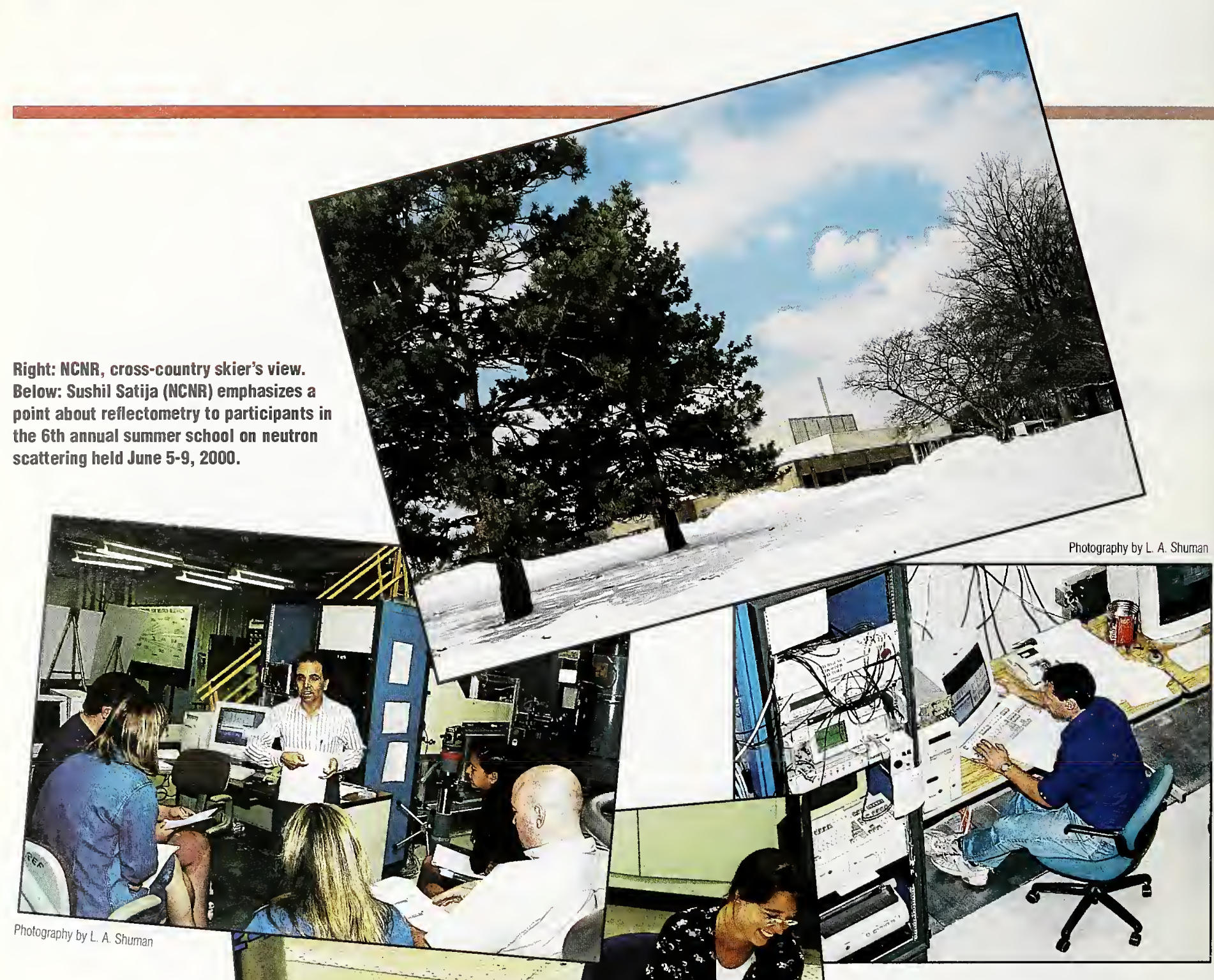

Above: Jeremy Cook (NCNR) at the control station of the Disk Chopper Spectrometer, which is highlighted in this issue. Left: Uta Dieregsweiler and Judith Wölk (U. Köln) measuring scattering from aerosols at the NG-7 $30 \mathrm{~m}$ SANS.

Right: Rob Dimeo (NCNR) examines the cryostat jacket inside the chamber of the backscattering spectrometer. Arrayed on Debye-Scherrer rings behind him are Si (111) analyzer crystals. 


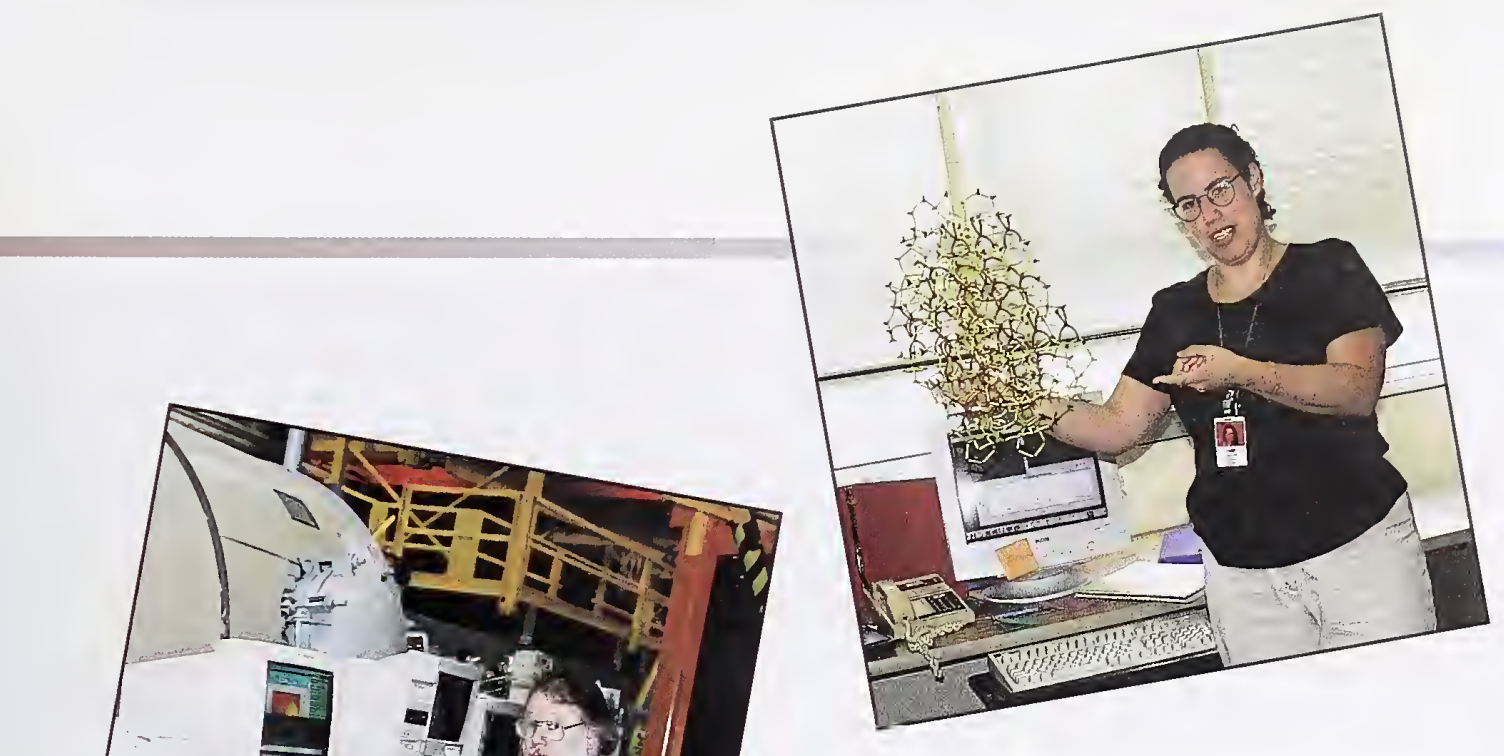

Above: Barbara Reisner (James Madison U.) makes a salient point using a model of a zeolite. Below: Rolf Zeisler and Heather Chen-Mayer (NCNR) prepare for pulsed neutron beam PGAA measurements.

Above: John McCarthy (ORNL) watches a pattern emerge at the NG-3 $30 \mathrm{~m}$ SANS.
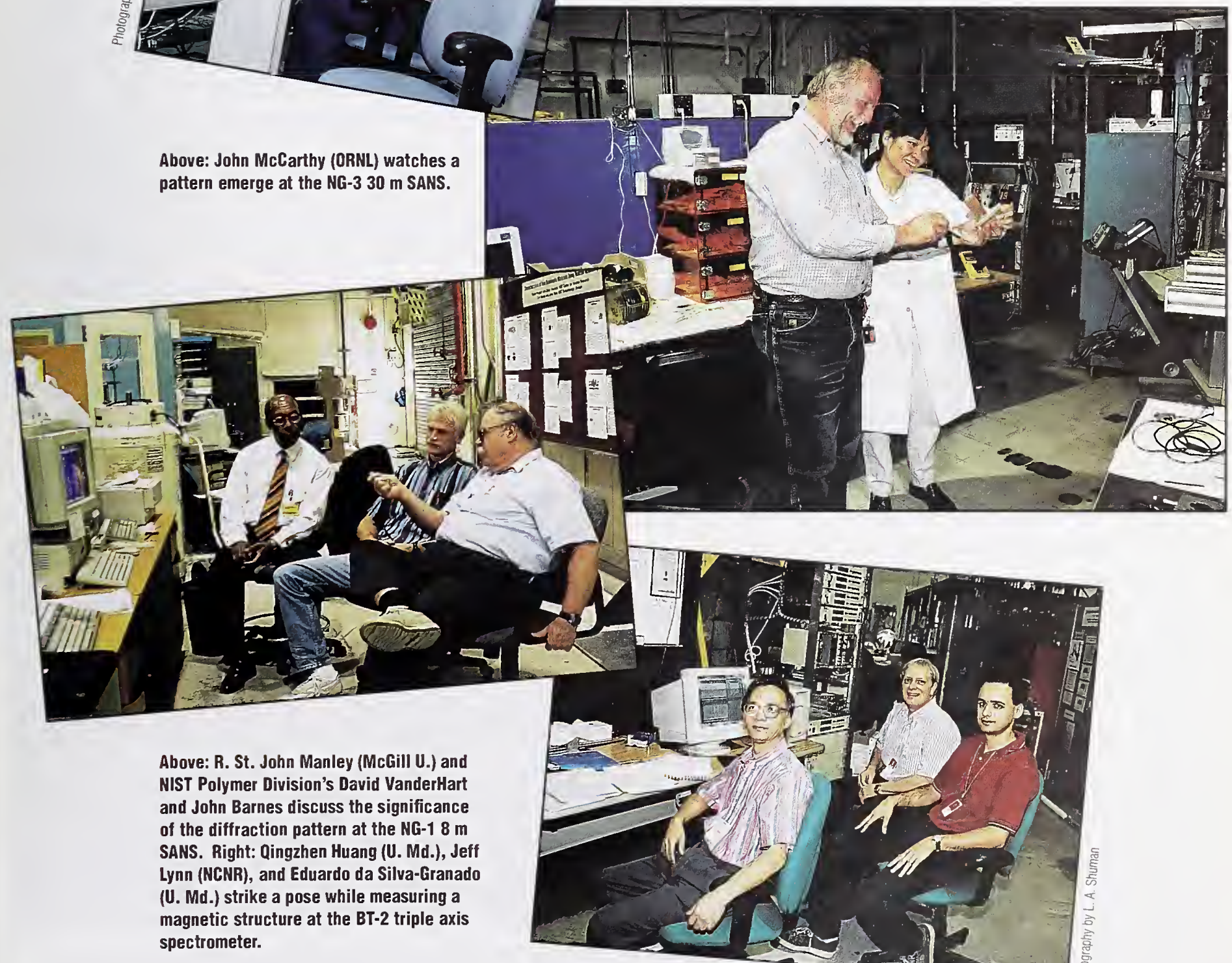


\section{PHASE-CONTRAST IMAGING WITH NEUTRONS}

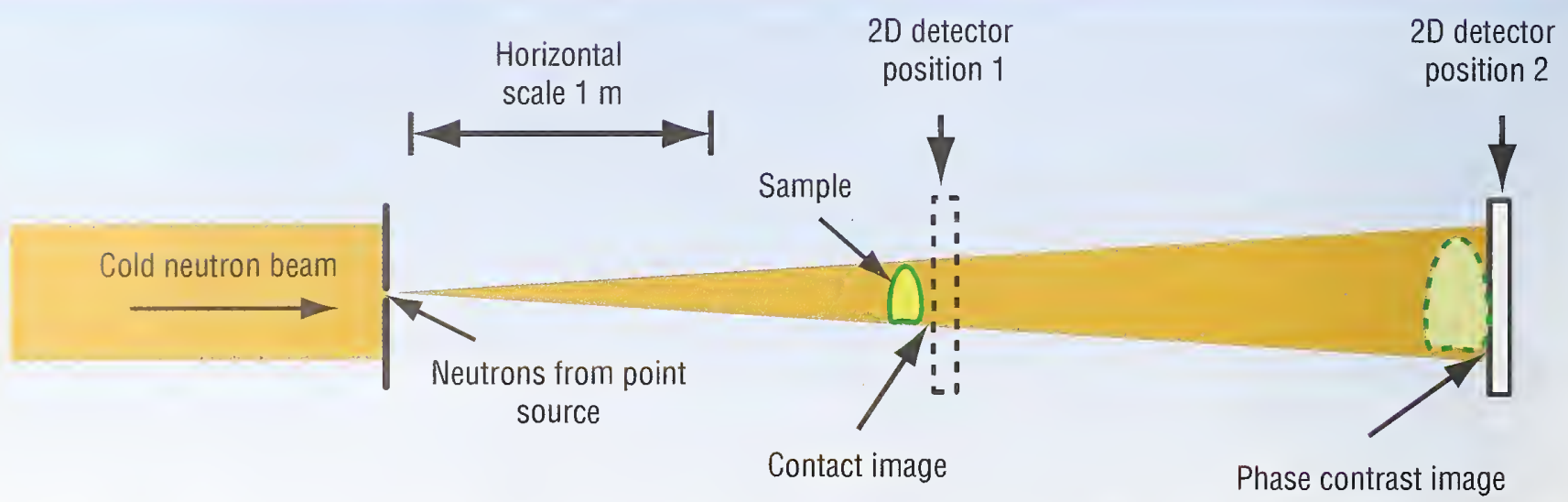

FIGURE 1. Experimental layout.

n-line phase-contrast images have been observed with many

forms of radiation. Here we demonstrate that such images can be observed very simply with neutrons as well. Measurement of the phase of a neutron wave conventionally requires a delicate setup, carefully reducing interference-destroying vibrations of the parts through which the beam passes. Here we describe an alternative method that only requires measurements of intensity to extract phase information, and thus bypasses the need for the precise setup used in a conventional interferometric measurement.

A schematic of the setup used for the present experiment is shown in Fig.1. To give them the required transverse coherence, monochromatic neutrons pass through a pinhole onto the sample. A 2-D CCD-type neutron camera then records the transmitted beam intensity through the sample at some distance from it (plane 2 in Fig. 1.) This downstream image is called the "phase-contrast image" because it is enhanced by Fresnel diffraction, since interference due to phase differences corresponding to edge effects has had a chance to develop. The radiographic image recorded immediately after the sample (plane 1 in Fig. 1) is called the "contact image," and essentially contains a shadow of the object.

The contact and phase-contrast images of the bullet-shaped lead sinker, aligned with its axis perpendicular to the beam, taken with a $0.433 \mathrm{~nm}$ beam at the NCNR interferometer beam line NG-7 are shown in Figs. $2 \mathrm{a}$ and $2 \mathrm{~b}$. These are direct images, corrected only for the empty beam profile. In the normal radiograph (Fig. 2a) the extent and internal details of the sample structure are not clearly visible because of very low scattering and absorption. However, in Fig. $2 b$ interference has enhanced the image intensity contrast at the edges of the sample and at boundaries within the imperfections at the tip, rendering them clearly visible. Contact and phase contrast images of a wasp are shown in Figs. $2 \mathrm{c}$ and 2 d, respectively. Notice that in the phase-contrast image all the delicate and thin organs of the wasp, e.g., antennae, leg segments, and wings, become visible. Since this method is so simple, many applications investigating internal features not otherwise visible are now possible.

Turning attention now to extracting phase information, after the beam has passed through the sample, particularly for an object offering little absorption or scattering, refractive variations within the sample still cause the beam's phase to be modulated. As a result, the radiation intensity transverse to the direction of propagation is redistributed. By measuring these intensity changes alone one can retrieve the phase density profile of the sample without using an interferometer. The mathematical basis for this phase retrieval

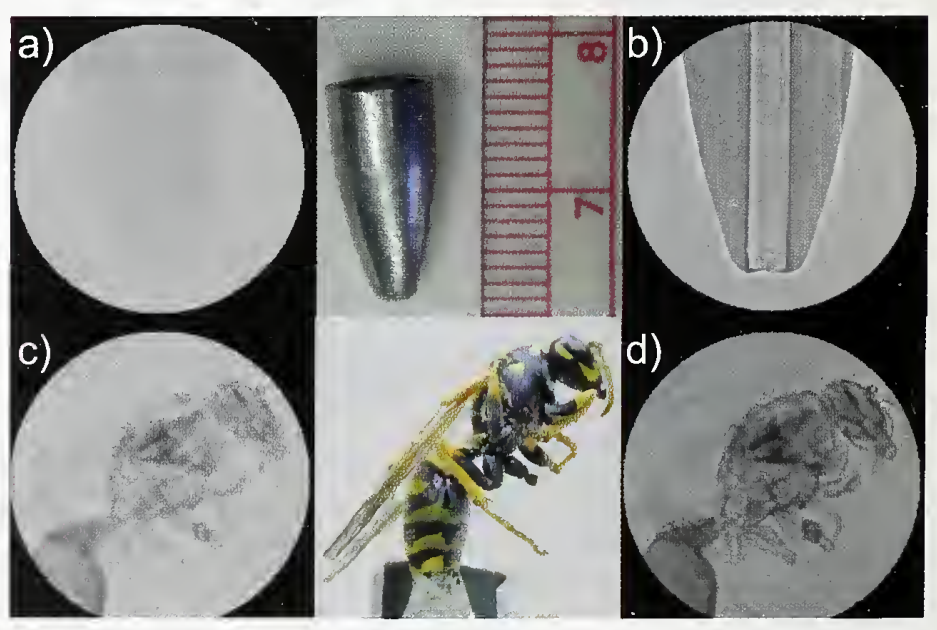

FIGURE 2. (a) contact and (b) phase contrast images of a lead sinker shown between them. (c) contact and (d) phase contrast images of a wasp. 
D.L. Jacobson, M. Arif and S.A. Werner Physics Laboratory

lonizing Radiation Division

National Institute of Standards and Technology

Gaithersburg, MD 20899-8461
B.E. Allman, P.J. McMahon, K.A. Nugent,

and D. Paganin

School of Physics

The University of Melbourne

Victoria 3010, Australia technique is based on the quantum mechanical analogue of the socalled "Transport of Intensity Equation (TIE)" of a wave

$\sqrt{I\left(\overrightarrow{r_{\perp}}, z\right)} \exp \left(\mathrm{i} \phi\left(\overrightarrow{r_{\perp}}, z\right)\right) \exp (i 2 \pi z / \lambda)$, with irradiance, $I\left(\overrightarrow{r_{\perp}}, z\right)$ and phase $\phi\left(\vec{r}_{\perp}, z\right)$ transverse to the beam $[1,2]$ :

$$
\frac{2 \pi \partial I\left(\overrightarrow{r_{\perp}}, 0\right)}{\lambda \partial z}=-\nabla_{\perp} \cdot\left[I\left(\overrightarrow{r_{\perp}}, 0\right) \nabla_{\perp} \phi\left(\overrightarrow{r_{\perp}}, 0\right)\right] \quad(1) .
$$

The TIE allows one to make a quantitative determination of the phase because the intensity of the propagated wave in a given transverse plane downstream is dependent on the intensity and phase upstream [3]. This technique is well developed for $\mathrm{x}$-rays and electrons. Very beautiful experiments have been performed using both of these radiation types $[4,5,6]$.

We recently carried out a series of experiments [7], for the first time with neutrons, to measure the phase modulated intensity changes caused by a sample and to quantify the observed phase profile using this technique. The experiments were performed at NG-7 and also at NG-0. NG-0 is a curved neutron guide providing a polychromatic neutron beam with the Maxwellian peak centered around $0.432 \mathrm{~nm}$. At the NG-7 guide a PG(002) crystal was used to extract a beam in the range of $0.235 \mathrm{~nm}$ to $0.475 \mathrm{~nm}$.

Both contact and phase-contrast images were recorded in order to obtain the derivative on the left side of Equation (1). The phase-contrast image was taken with the CCD placed $1.5 \mathrm{~m}$ to $1.8 \mathrm{~m}$ from the sample. The images from the CCD are downloaded to a computer and the centroids of the neutron events are deter-

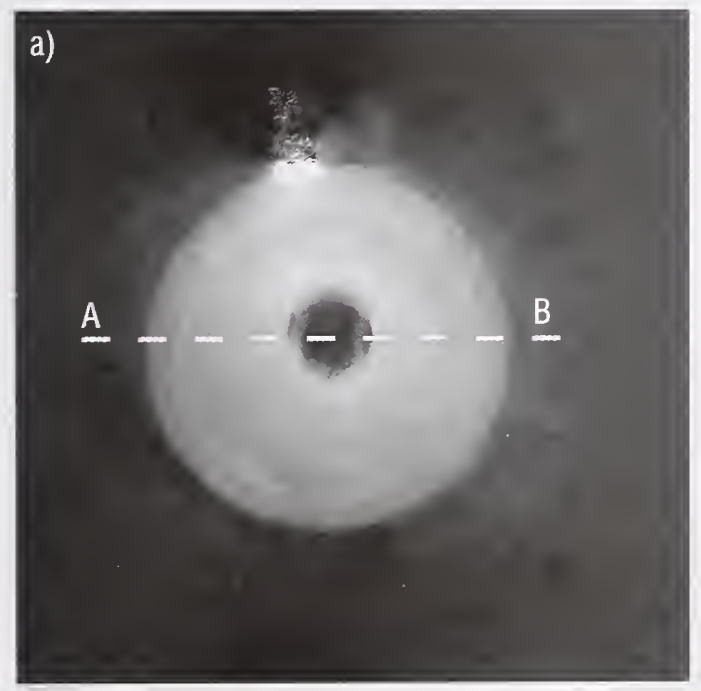

mined by hardware processing, achieving a best-case resolution of about $60 \mu \mathrm{m}$.

Figure 3a shows an image constructed from such an analysis of the lead sinker (of Fig. 2) whose longitudinal axis is nearly aligned with the incident beam. In Fig. $3 \mathrm{~b}$ is plotted a profile of the retrieved phase along $\mathrm{AB}$ (blue curve). This profile was obtained through Fourier processing of the data. The predicted phase profile (dotted curve) determined from the sample geometry and orientation is in excellent quantitative agreement with the experimental data.

This phase retrieval technique works with a polychromatic beam and is not constrained by low flux. We plan to extend both of these types of measurements using polarized and very cold neutrons to study magnetic domain structures, interface boundaries and density variations in multi-layer thin films. We also plan to carry out tomographic measurements in the near future.

\section{REFERENCES}

[1] M.R. Teague, J.Opt.Soc.Am. 73, 1434 (1983).

[2] T. E. Gureyev, A. Roberts, and K. A. Nugent, J. Opt. Soc. Am. A 12, 1942 (1995).

[3] D. Paganin and K.A. Nugent, Phys. Rev. Lett. 80, 2586 (1998).

[4] A. Barty, K. A. Nugent, D. Paganin, and A. Roberts, Opt. Lett. 23, 817 (1998).

[5] B. E. Allman, P. J. McMahon, J. B. Tiller, K. A. Nugent, D. Paganin, A. Barty, I. McNulty, S. P. Frigo, S. Wang, and C.C. Retsch, J.Opt. Soc. Am A 17, 1732 (2000).

[6] K. A. Nugent, T. E. Gureyev, D. Cookson, D. Paganin, and Z. Barnea, Phys. Rev. Lett. 77, 2961 (1996).

[7] B. E. Allman, P. J. McMahon, K. A. Nugent, D. Paganin. D. L. Jacobson, M. Arif, and S. A. Werner, Nature 408, 158 (2000).

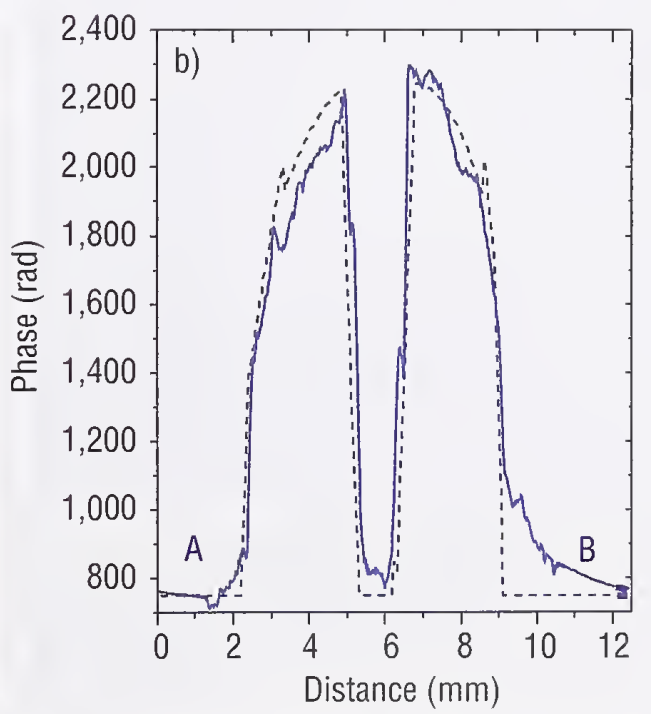

FIGURE 3. a) reconstructed image of lead sinker along beam direction; b) measured (blue) and computed (dotted) phase variations along line $A B$ in a). 


\section{FILTER-ANALYZER NEUTRON SPECTROMETER (FANS)}

$\mathbf{N}$ eutron vibrational spectroscopy (NVS) is an invaluable technique for probing vibrational dynamics. In particular, the typical range of energies accessible with reactor-based neutrons spans the region of important lattice and molecular vibrations. Moreover, the unique nature of the neutron-nucleus interaction permits the observation of all vibrational modes in an NVS experiment, not just those that satisfy appropriate symmetry-based selection rules as in photon spectroscopies. NVS is particularly useful for characterizing hydrogenous materials since the incoherent scattering cross section for hydrogen is much larger than for virtually all the other elements.

Over the past twenty years, neutron vibrational spectra have been collected for a broad array of both hydrogenous and nonhydrogenous systems utilizing a first-generation Filter-Analyzer Neutron Spectrometer (FANS) located at BT-4. Although this instrument possessed an excellent signal/noise ratio, the measured scattering intensities were limited by a relatively small detector solid angle. Moreover, the BT-4 beam time was typically shared with a triple-axis instrument. A second-generation FANS is currently being constructed at BT-4 in two phases in order to obtain a dedicated

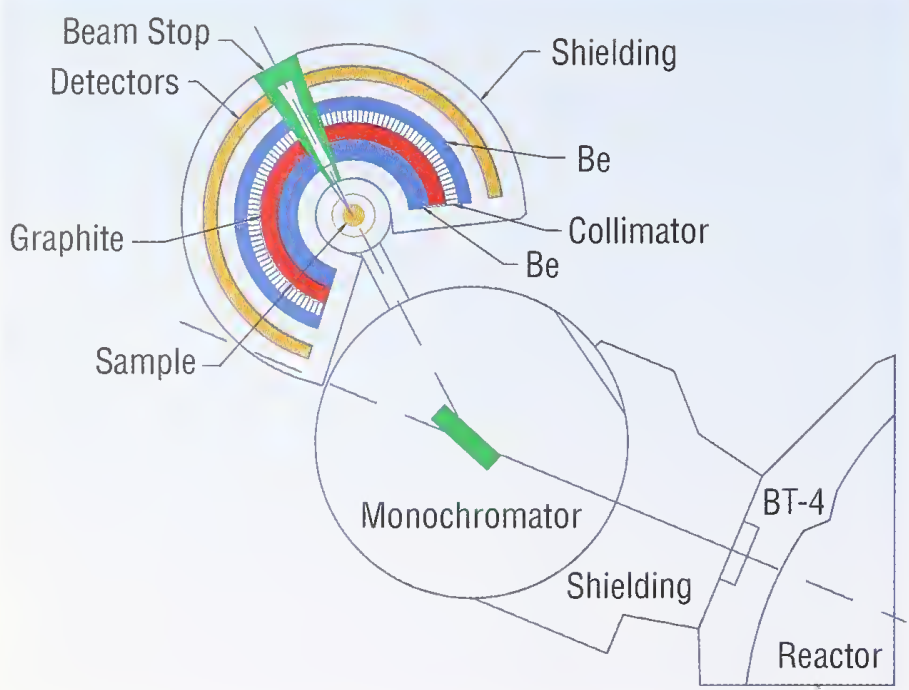

FIGURE 1. Schematic diagram of the final configuration of FANS. Presently installed phase I includes the filters to the right of the beam.
FANS spectrometer with an enhanced measurement sensitivity of about two orders of magnitude. This instrument will ultimately overcome the classic objections to NVS, namely the requirement for much larger samples and longer counting times than are typical of infrared and Raman spectroscopies. Phase I of this second-generation instrument (FANS-I) is now complete, yielding a twenty-fold increase in sensitivity with another factor of at least five anticipated upon completion of phase II.

Figure 1 illustrates the FANS layout. Using either a $\mathrm{Cu}(220)$ or pyrolitic graphite (PG) (002) monochromator covers an accessible

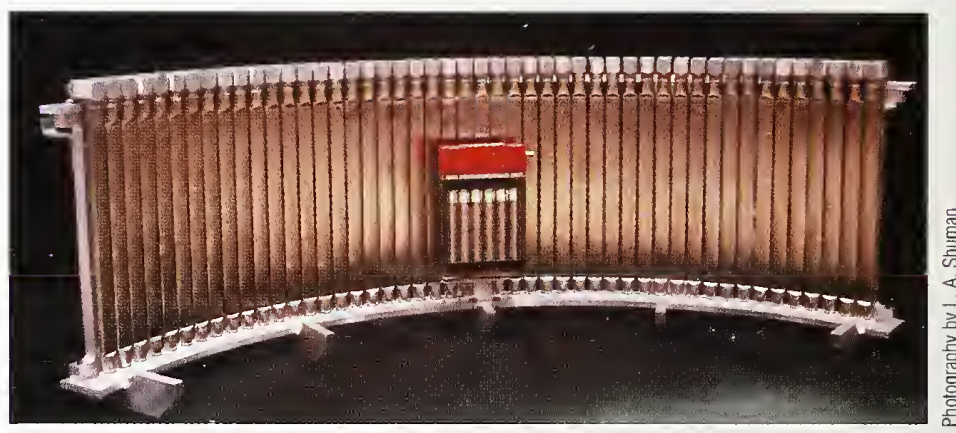

FIGURE 2. Comparison of the larger phase-I and original FANS detector banks.

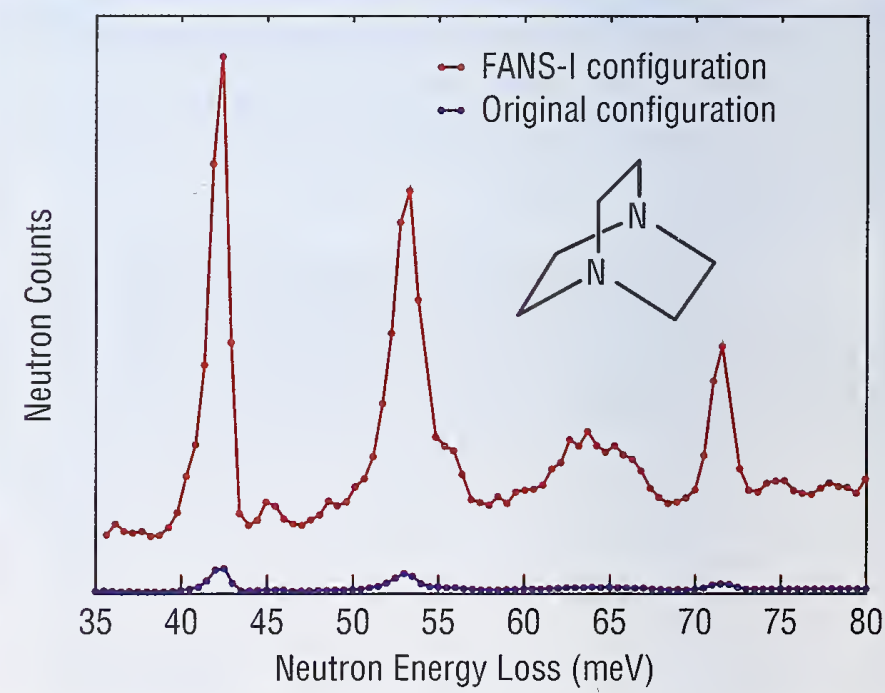

FIGURE 3. Comparison of the neutron vibrational spectrum for triethylene diamine $\left(\mathrm{C}_{2} \mathrm{H}_{4}\right)_{3} \mathrm{~N}_{2}$ measured with FANS-I vs. that measured with the original configuration. 
D. A. Neumann and T. J. Udovic NIST Center for Neutron Research National Institute of Standards and Technology Gaithersburg, MD 20899-8562
P. Papanek

NIST Center for Neutron Research

National Institute of Standards and Technolog Gaithersburg, MD 20899-8562 and

Department of Materials Science and Engineering University of Pennsylvania

Philadelphia, PA 19104

\section{L. Klein}

Department of Chemistry

University of Pennsylvania

Philadelphia, PA 19103

J. E. Fischer

Department of Materials Science and Engineering

University of Pennsylvania

Philadelphia, PA 19104

\section{A. K. Cheetham}

Materials Department

University of California at Santa Barbara

Santa Barbara, CA 93106 energy range of $\approx 5 \mathrm{meV}$ to $250 \mathrm{meV}$ with options for precollimations of $60^{\circ}, 40^{\prime}$, or $20^{\circ}$ and postcollimations of $40^{\prime}, 20^{\prime}$, or $10^{\prime}$.

The intensity improvement of the phase-I instrument is due to the twenty-fold increase in detector solid angle provided by a much larger detector bank (see Fig. 2). The accompanying analyzer filter consists of a Be-PG-Be layered arrangement cooled with liquid nitrogen to enhance transmission of the low energy neutrons. Such a low-bandpass filter provides a best resolution at the lowest energy transfers of $\approx 1.1 \mathrm{meV}$ FWHM.

The first measurements during the spring of 2000 confirmed the magnitude of the expected gains. For example, Fig. 3 displays the low-temperature neutron vibrational spectrum for triethylene diamine measured using the new FANS-I configuration compared with that measured using the original configuration under identical beam collimations and measurement times. This gain in intensity is accompanied by a somewhat improved signal/noise ratio.

The enhanced capabilities of the FANS-I instrument have already been demonstrated for a variety of materials including protonic conductors, organic solids, metal hydrides, carbon nanotubes, and metal oxides. These experiments confirmed substantial reductions in required sample size and/or measurement time. For exam-

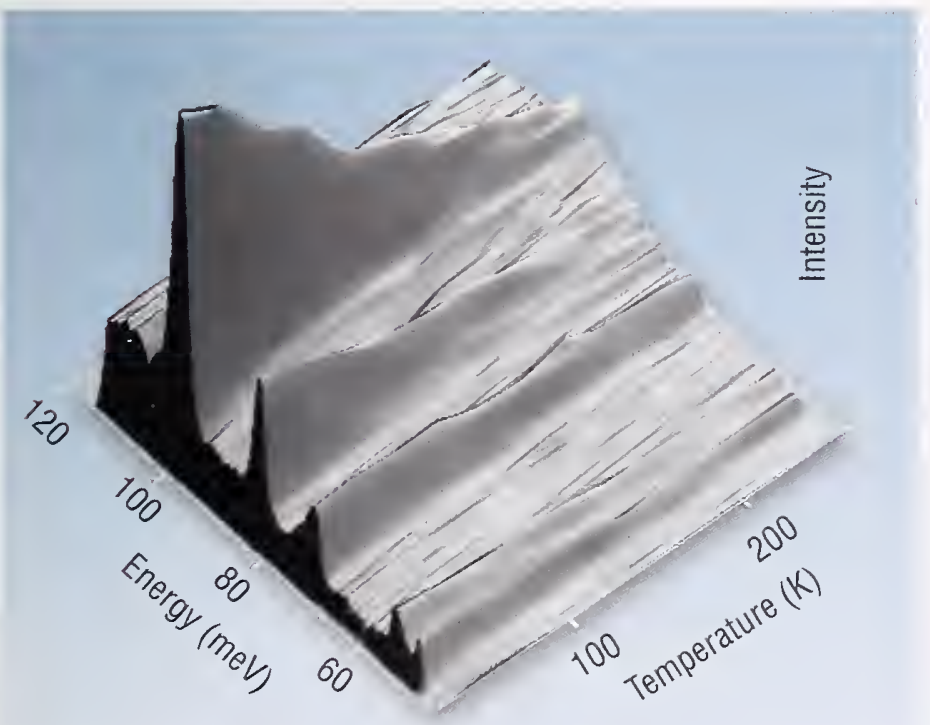

FIGURE 4. Temperature dependence of the NV spectrum of $\mathrm{RbH}\left(\mathrm{SO}_{4}\right)_{0.81}\left(\mathrm{SeO}_{4}\right)_{0.19}$. (Data collected by T. Yildirim, NCNR.) ple, Fig. 4 displays the temperature dependence of the NV spectrum for $\mathrm{RbH}\left(\mathrm{SO}_{4}\right)_{0.81}\left(\mathrm{SeO}_{4}\right)_{0.19}$, a protonic conductor oxide with lattice protons that become mobile at temperatures less than $473 \mathrm{~K}$. Vibrational spectra were collected with FANS-I at nine different temperatures in less than one day, a feat not possible using the original instrument. It proved particularly interesting that the energy of the mode near $100 \mathrm{meV}$ decreases while that of the mode near 83 $\mathrm{meV}$ increases with increases in temperature concomitant with rapid decreases in both peak intensities. Indeed, these proton-related features largely disappear at temperatures as low as $200 \mathrm{~K}$, indicating that there is significant proton motion even at this low temperature.

Figure 5 shows the FANS-I spectrum for solid 3-nitrophenol $\left[\mathrm{HO}\left(\mathrm{C}_{6} \mathrm{H}_{4}\right) \mathrm{NO}_{2}\right]$ compared with a GAUSSIAN calculation for the isolated molecule. This spectrum exemplifies the overall quality and high-resolution capabilities enabled by the marked improvement in sensitivity. There is fair agreement between calculation and experiment for many features in the vibrational density of states, although it is clear that significant intermolecular hydrogen bonding interactions in the solid cause strong perturbations in the $\mathrm{OH}$ wagging mode predicted near $45 \mathrm{meV}$ for the isolated molecule.

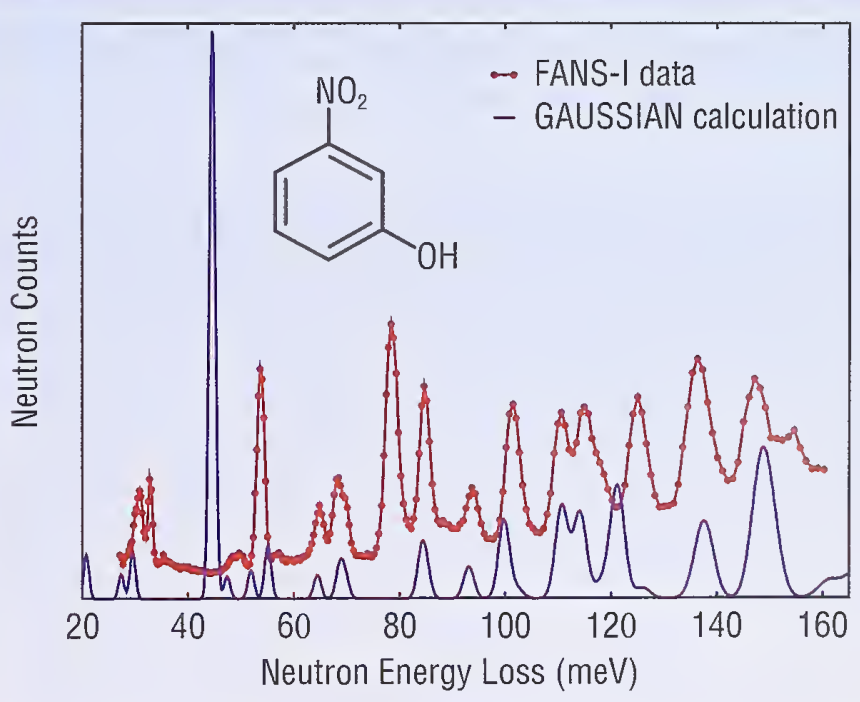

FIGURE 5. NV spectrum for solid 3-nitrophenol $\left[\mathrm{HO}\left(\mathrm{C}_{6} \mathrm{H}_{4}\right) \mathrm{NO}_{2}\right]$ at $10 \mathrm{~K}$ compared with a GAUSSIAN calculation (b3lyp/6-316*) for the isolated molecule. (Data collected by T. Heilweil, NIST Physics Laboratory, and C. M. Brown, U. Md. and NCNR.) 


\section{THE NCNR DISK CHOPPER TIME-OF-FLIGHT SPECTROMETER (DCS)}

$\mathbf{T}$ he recently commissioned Disk Chopper Spectrometer (DCS)

is a versatile state-of-the-art instrument that is primarily intended for studies of diffusional processes and low energy excitations in materials. It has no equal in North America and is fully competitive with comparable instruments in Europe.

The spectrometer is shown schematically in Fig. 1. Following a tapered offset guide assembly ("neutron optical filter") that removes almost all of the $\gamma$-rays and high energy neutrons from the reactor beam [1], seven phased disk choppers supply monochromatic bursts of neutrons at the sample position. Three parallel banks of 6 atmosphere ${ }^{3} \mathrm{He}$ detectors, of $400 \mathrm{~mm}$ active length, are placed $4010 \mathrm{~mm}$ from the sample position, and $\approx 90 \%$ of the space between the sample and the detectors is argon-filled. Each of the 913 rectangular cross section detectors subtends $\approx 0.5^{\circ}$ in the scattering plane. The central bank provides continuous angular coverage from $-30^{\circ}$ to $-5^{\circ}$ and from $5^{\circ}$ to $140^{\circ}$. The overall detector coverage is $\approx 0.65 \mathrm{sr}$, double that of the IN5 spectrometer at the Institut Laue Langevin, Grenoble. Presently fitted with $\mathrm{a} \approx 50 \mathrm{~mm}$ long room temperature beryllium filter, the instrument operates at wavelengths greater than $\approx 4.1 \AA$. The beryllium will shortly be replaced with an assembly of cold oriented graphite, $\approx 100 \mathrm{~mm}$ in length, permitting measurements down to $\approx 2.3 \AA$. (Wavelengths near $3.33 \AA$ and 6.67 $\AA$ will be unavailable.) The first two and last two choppers are fitted with three slots of different widths, enabling a choice among three "resolution modes" at a given wavelength and master chopper speed.
The neutron current density at the sample and the energy resolution width at the detectors are shown in Figs. 2 and 3 respectively. In designing and building the DCS, great care has been taken to ensure that distances and detector locations are accurately known. The stability of the chopper phasing results in a resolution lineshape with a sharp leading edge. The sample area is easily accessed at beam level and from above. The data acquisition system has been carefully designed and is extremely reliable. We plan to modify it so that crude pulse height spectra can be extracted. The software is user-friendly and will be improved as time permits. Planned improvements, apart from the crystal filter replacement, include removal of the two innermost reflecting plates within the guides after the first chopper; this will increase the flux with little reduction in versatility. With the new cold source and these optics improvements we anticipate a threefold improvement in flux within the next year.

The first officially approved experiment using the DCS was a comparative study [2] of native bovine -lactalbumin (BLA) and -lactalbumin in a "molten globular" state (MBLA); the latter state is partially folded and compact, with native-like secondary structure but lacking the side-chain packing that characterizes the native state. Molten globules play an important role in understanding protein folding mechanisms, and molten globules also participate in important cellular functions. The DCS measurements (Fig. 4) confirm and extend previous results $[3$, and see also the article by $\mathrm{Z}$. $\mathrm{Bu}$ et al. on

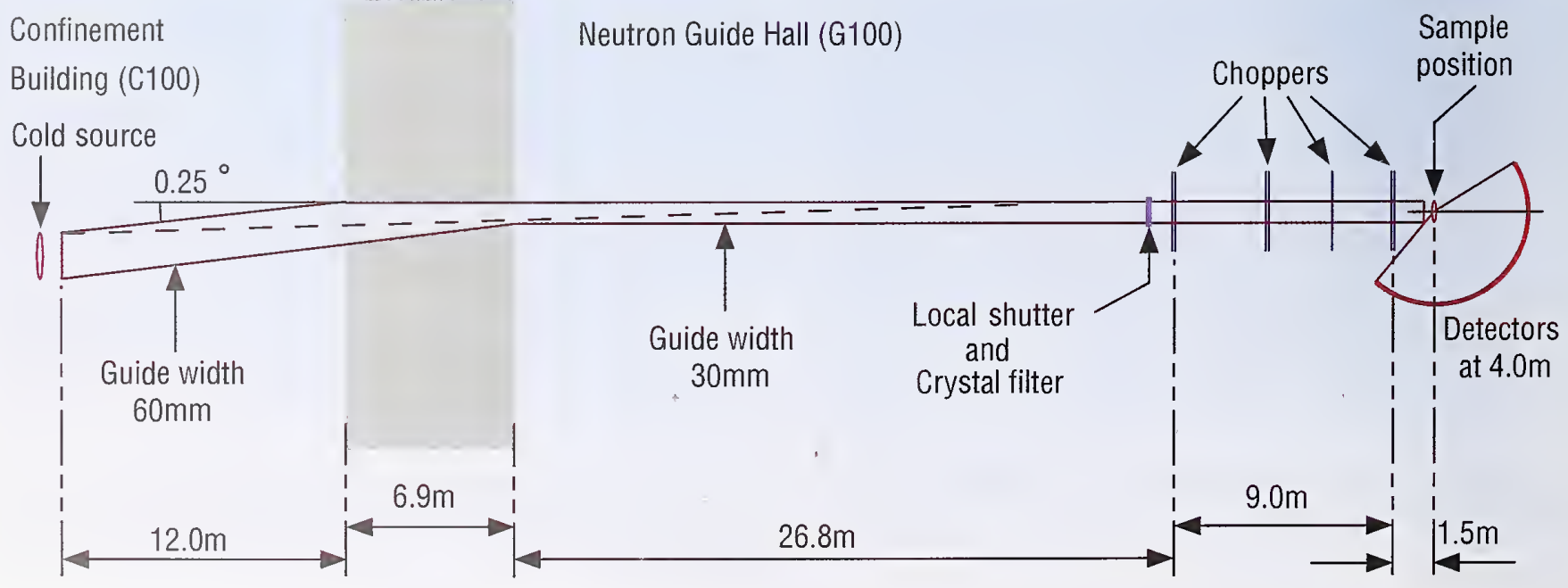


John Copley

NIST Center for Neutron Research

National Institute of Standards and Technology

Gaithersburg, MD 20899-8562
Jeremy Cook

NIST Center for Neutron Research

National institute of Standards and Technology

Gaithersourg, MD 20899-8562

and

University of Maryland

College Park, MO 20742
Felix Altorfer

Paul Scherrer Institut

CH-5232 Villigen PSI

Switzerland p. 20 of this report] obtained with the Fermi chopper spectrometer (FCS): the improved energy resolution of the DCS adds confidence to the earlier results, especially for BLA. The structural differences between BLA and MBLA are reflected in the dynamics on time scales of order $10 \mathrm{ps}$ to $100 \mathrm{ps}$, whereas complementary measure-

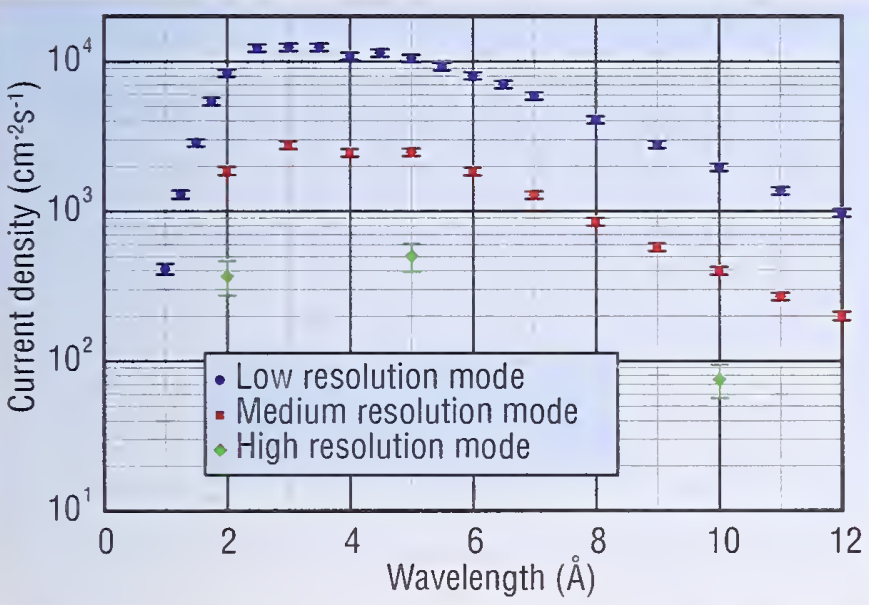

FIGURE 2. The neutron current density at the sample position with all choppers spinning at $20000 \mathrm{rpm}$. Measured values are shown for the three "resolution modes" of the instrument.

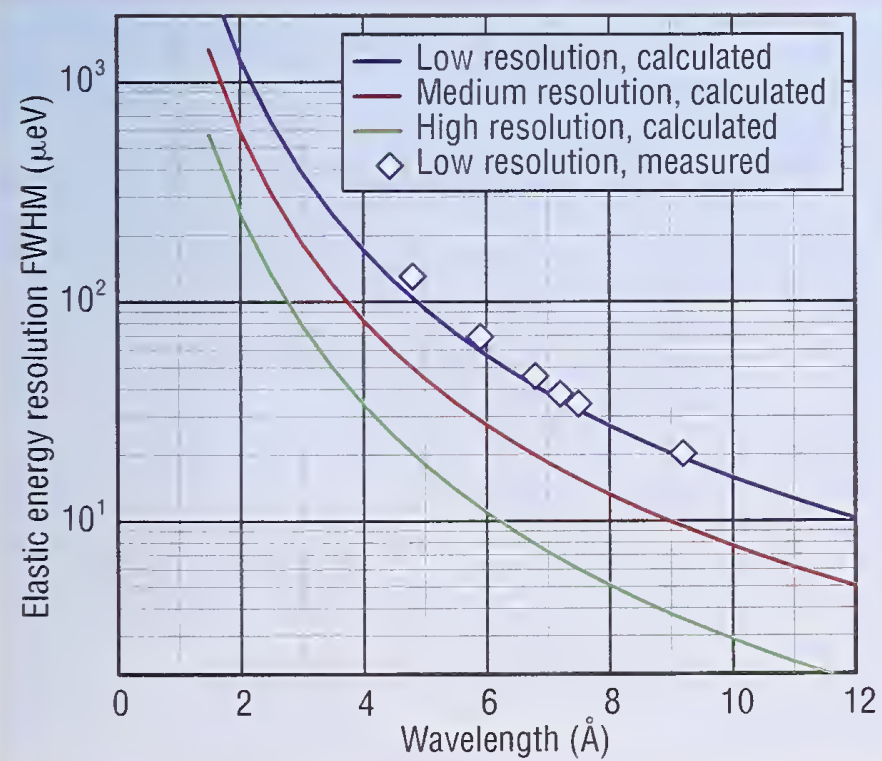

FIGURE 3. Calculated resolution widths (full widths at half maximum height) for the three "resolution modes" of the instrument. Some experimental widths are also shown. ments have shown that the high frequency dynamics are virtually unchanged.

The moniker "boson peak" has of late been associated with an excess feature that shows up in the vibrational density of states of many materials, generally in the Debye region, between $1 \mathrm{meV}$ and $10 \mathrm{meV}$. While the molecular origin of the peak is unclear, its characteristic energy suggests that it is a collective excitation between the low energy acoustic modes and localized high energy optic modes (representing local bond vibrations, librations, bendings, etc.). In a recent experiment [4] the thermal softening of the boson peak was studied in detail in a polyester carbonate copolymer that had already been extensively studied using the FCS and the NCNR backscattering spectrometer. The DCS is particularly well suited for such a study because of the large solid angle of detectors and the large beam size. The analysis of the data is in progress.

Other materials recently studied using the DCS include carbon nanotubes and superionic proton conductors.

\section{REFERENCES}

[1] J.R.D. Copley, J. Neutron Res. 2, 95 (1994).

[2] Z. Bu et al., private communication.

[3] Z. Bu, D. A. Neumann, S.-H. Lee, C. M. Brown, D. M. Engelman, and C. C. Han, J. Mol. Biol. 301, 525 (2000).

[4] C. Soles et al., private communication.

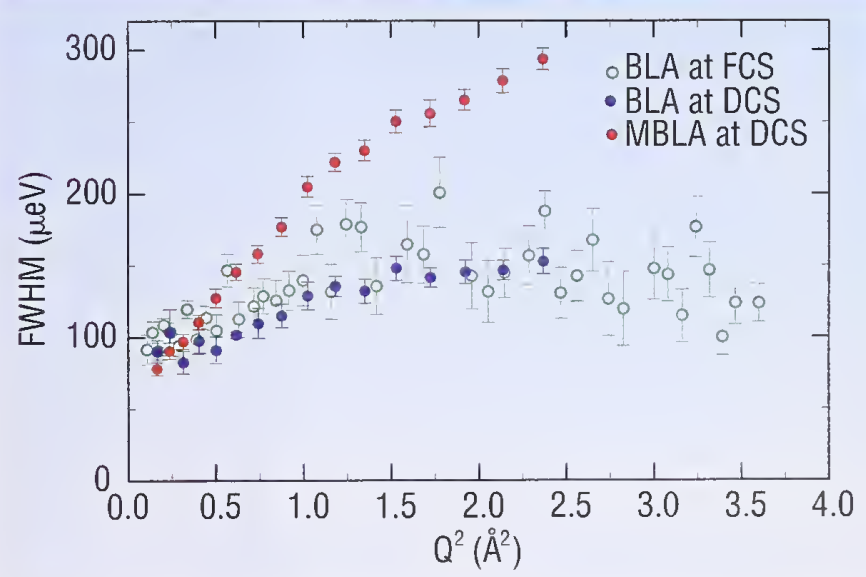

FIGURE 4. The $Q$ dependence of the full-width at half maximum height of the quasielastic Lorentzian peak for native bovine $\alpha$-lactalbumin (BLA) and for molten globular bovine $\alpha$-lactalbumin (MBLA) in 8M urea [2]. Results [3] for BLA from the Fermi chopper spectrometer (FCS) are shown for comparison. 


\section{ULTRA-HIGH RESOLUTION SMALL-ANGLE NEUTRON SCATTERING (USANS)}

A perfect crystal diffractometer (PCD), shown in Fig.1, for ultrahigh resolution small-angle neutron scattering (USANS) measurements is now in operation at the thermal neutron beam port, BT-5. The PCD increases the maximum size of features accessible with the NCNR's $30 \mathrm{~m}$ long, pinhole collimation SANS instruments by nearly two orders of magnitude, from $\approx 10^{2} \mathrm{~nm}$ to $10^{4} \mathrm{~nm}$.

The PCD is a Bonse-Hart type instrument with large triplebounce, channel-cut Si (220) crystals as monochromator and analyzer. The perfect crystals provide high angular resolution while the multiple reflections suppress the "wings" of the beam profile, improving the signal-to-noise ratio to values comparable to that obtained with pinhole instruments. This technique, widely utilized for $\mathrm{x}$-rays for many years, has only recently been successfully adapted for neutrons [1] as dynamical diffraction effects arising from the deep penetration of neutrons in thick perfect crystals have become better understood. Neutrons can, in effect, propagate through a thick crystal, and then reflect from the back-face of the crystal. The geometry of this second diffraction path allows part of the beam to bypass the second and third reflections. The design of the NCNR's PCD [2] successfully eliminates the single reflection path by adding shielding along the middle of the long face of each crystal between the first and third reflections (see inset in

Fig. 2). The additional shielding reduces the wings in the rocking curve by two orders of magnitude, resulting in a signal-to-noise ratio of $10^{5}$ at a minimum scattering vector $Q=0.0005 \mathrm{~nm}^{-1}$. Figure 2 shows typical rocking curves with and without shielding of the deleterious back-face reflection. The beam flux obtained for smaller samples is $3000 \mathrm{~cm}^{-2} \mathrm{~s}^{-1}$, while the maximum intensity obtained is $15000 \mathrm{~s}^{-1}$ when using the maximum $3 \times 5 \mathrm{~cm}^{2}$ beam size. The mainly fast neutron flat background $\left(\approx 0.15 \mathrm{~s}^{-1}\right)$ found at large angles is independent of beam size. The beam intensity will increase somewhat when the present perfect crystals are replaced by ones with a wider channel, and a gap in the middle of the long face, in order to increase the beam width to $4 \mathrm{~cm}$ with no contamination from single back-face reflections.

The measurement range of the PCD overlaps that of the NCNR's 30 m SANS instruments. Together they probe structure in materials over four orders of magnitude, from $\approx 1 \mathrm{~nm}$ to $10^{4}$ $\mathrm{nm}$. Combined measurements on these instruments will enable fuller characterization of hierarchical and highly anisotropic microstructures in materials, for example in fiber or clay impregnated nanocomposites. The PCD is part of the NIST/NSF Center for High Resolution Neutron Scattering (CHRNS) with up to two-thirds of the available beam time to be allocated by the NCNR's Program Advisory Committee to scientists and engineers who submit proposals for peer review.

The PCD USANS instrument can accept any ancilliary sam-

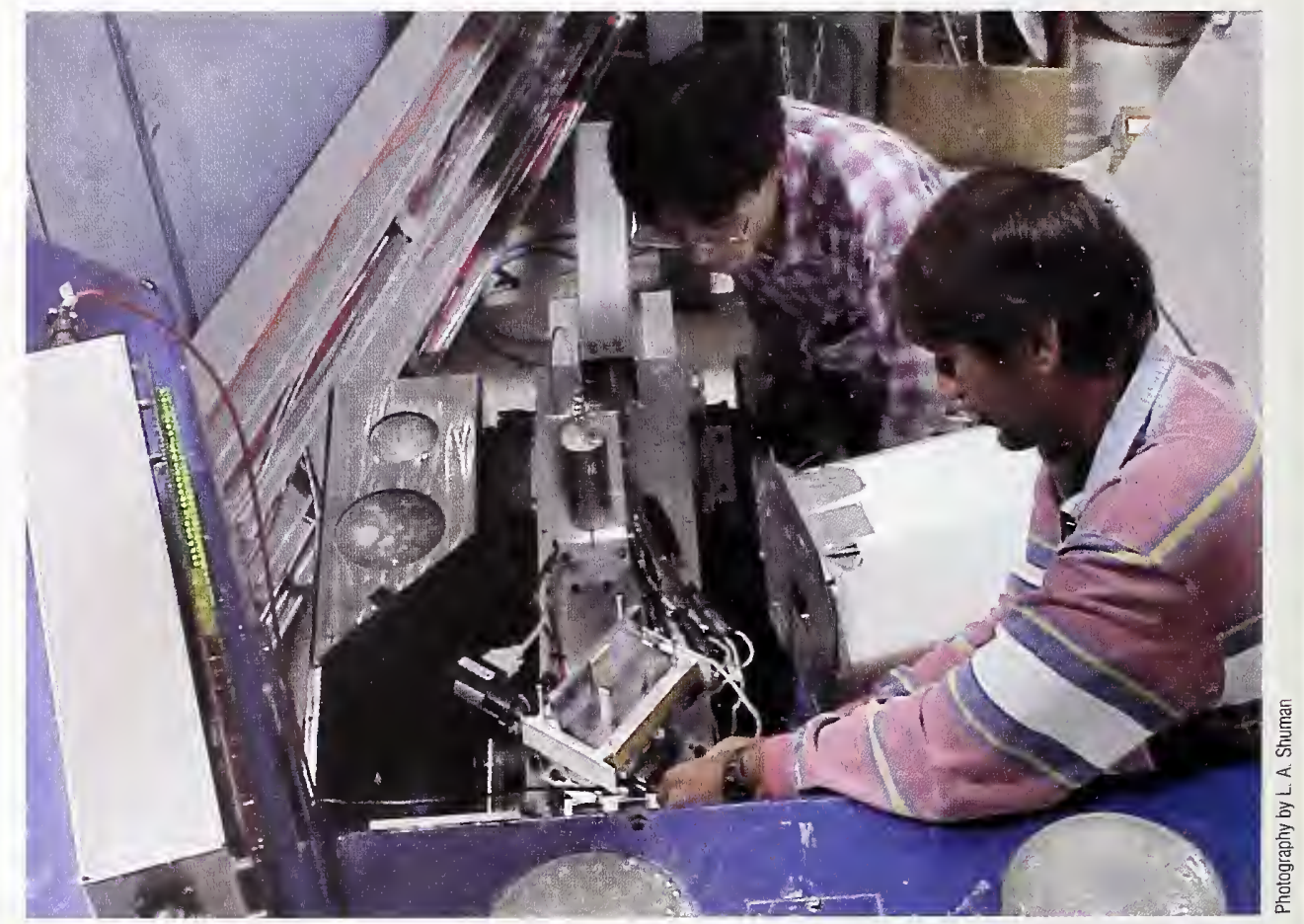

FIGURE 1. NCNR's Derek Ho (top) and John Barker load a sample at the PCD. The triple-bounce analyzer is visible in the center foreground of the picture. 


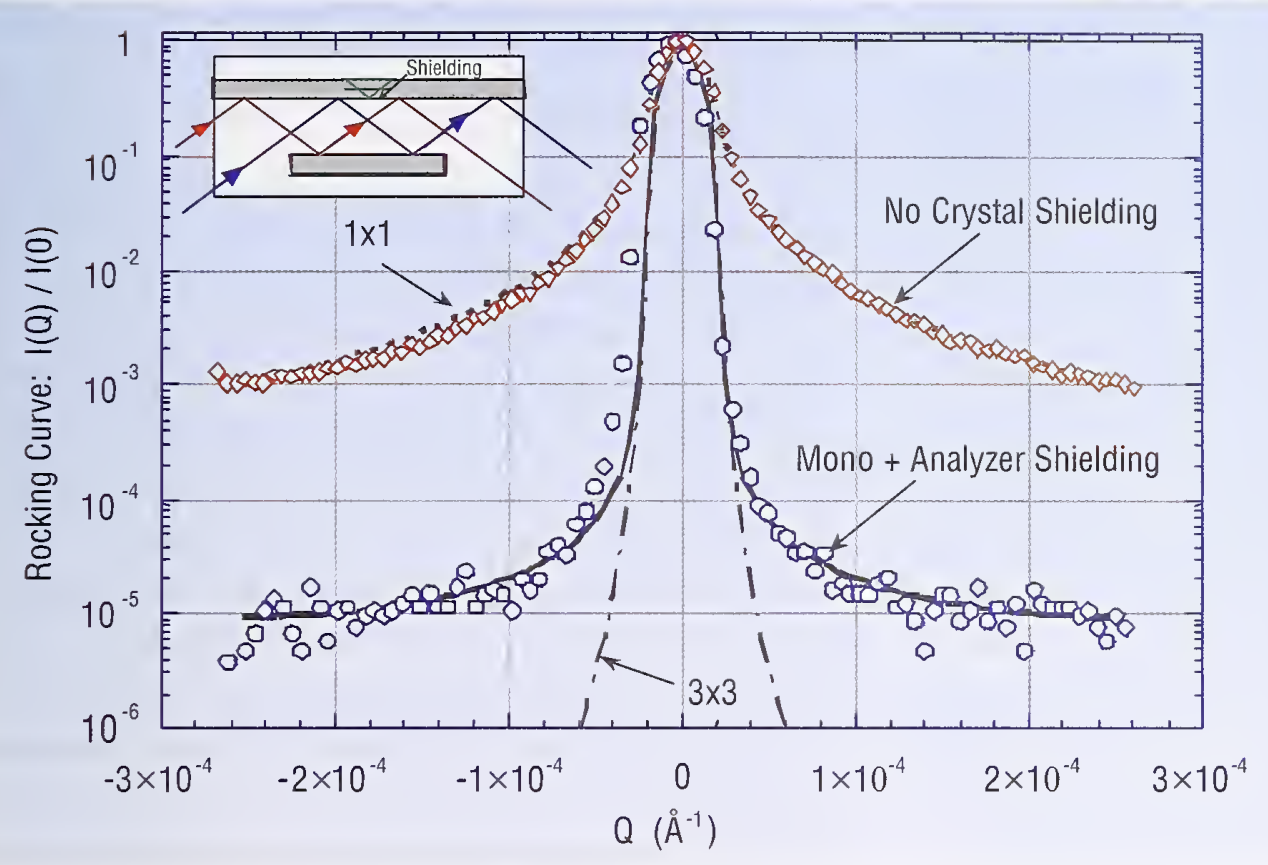

FIGURE 2. Rocking curves measured for the PCD USANS instrument. The diamond symbols are data taken before adding shielding to block back-face reflections from the triple-bounce, channel-cut monochromator and analyzer crystals. The circle symbols are data taken after adding such shielding. The dash-dot curve is the theoretical profile for a pair of triple-bounce perfect crystals. The solid line is the weighted sum of the theoretical profiles for $3 \times 3$ and $1 \times 1$ rocking curves, with weighting factors of 0.998 and 0.002 , respectively. The inset shows a schematic diagram of a channel-cut crystal with the shielding needed to remove the single reflection path from the back-face of the crystal.

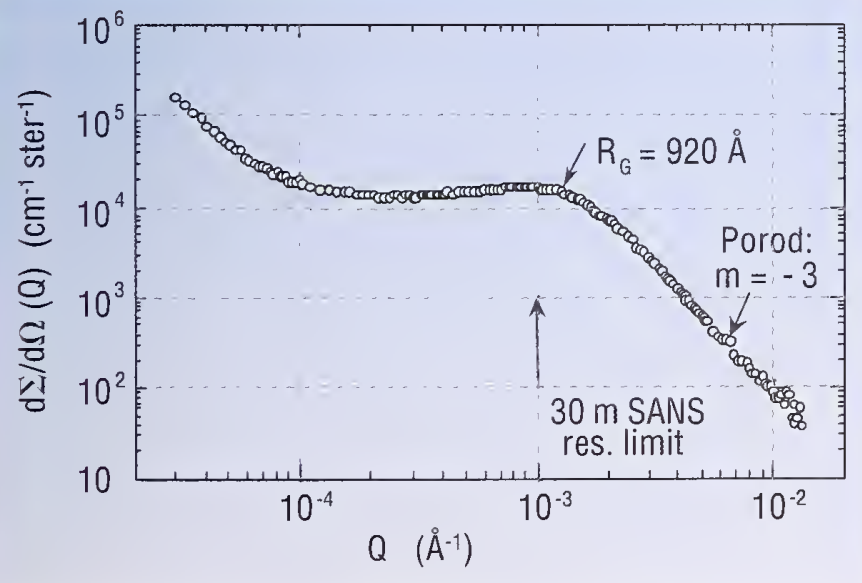

FIGURE 3. First USANS measurement obtained using new PCD instrument. The sample is a $2.5 \mathrm{~cm}$ thick commercially obtained poly(tetrafluoroethylene) plate. Scattering is predominately from residual sub-micron size pores $\{0.2 \%$ by volume) that survive the extrusion processing of the material. ple environment equipment that is used on the $30 \mathrm{~m}$ SANS instruments. Larger liquid sample cells and a dedicated two-position heating block $\left(30^{\circ} \mathrm{C}\right.$ to $\left.400{ }^{\circ} \mathrm{C}\right)$ are currently being designed to utilize the larger available beam size.

The first USANS measurement was made in May 2000 on a commercially obtained poly(tetrafluoroethylene) plate. The slitsmeared data are shown in Fig. 3. The data easily overlap the accessible $Q$-range the $30 \mathrm{~m}$ SANS instruments. Examples of material systems studied so far are: pigment aggregation in paint, clay aggregation structures in various solutions and polymer melts, pores in copper, hydrides in uranium, and large scale structures in controlled pore glasses.

\section{REFERENCES}

[1] M. Agamalian, G.D. Wignall, R. Triolo, J. Appl. Cryst. 30, 345 (1997).

[2] A.R. Drews, J.G. Barker, C.J. Glinka, M. Agamalian, Physica B 241-243, 189 (1998). 


\section{LIGAND DYNAMICS IN Mn[N(CN $\left.)_{2}\right]_{2}$ PYRAZINE}

0 ne of the major themes of solid state chemistry is the development of new molecular architectures with novel chemical and physical properties for applications such as chemical separation, catalysis, and magnetic devices. Compounds consisting of transition metal ions linked together by polydentate organic ligands are of particular interest because their properties can be tailored by judicious choice of the components. For example, changing the transition metal can alter both the bonding motif and magnetic properties of these systems. The properties of these materials can also be controlled by introducing ancillary $\pi$-conjugated ligands such as pyrazine (pyz), 4,4'-bipyridine and 2,2'-bipyridine. These molecular building blocks not only affect the spatial separation of the transition metal cations and the dimensionality of the crystal, but also modulate the superexchange interactions. For instance, of the many $\mathrm{Mn}\left[\mathrm{N}(\mathrm{CN})_{2}\right]_{2} L$ materials examined to date, only $L=$ pyrazine exhibits long-range magnetic order above $2 \mathrm{~K}$.

The structure of $\mathrm{Mn}\left[\mathrm{N}(\mathrm{CN})_{2}\right]_{2}$.pyz can be described as an inter-penetrating $\mathrm{ReO}_{3}$-like network with axially elongated $\mathrm{Mn}^{2+}$ octahedral and edges made-up of $\mu$-bonded $\left[\mathrm{N}(\mathrm{CN})_{2}\right]^{-}$anions and

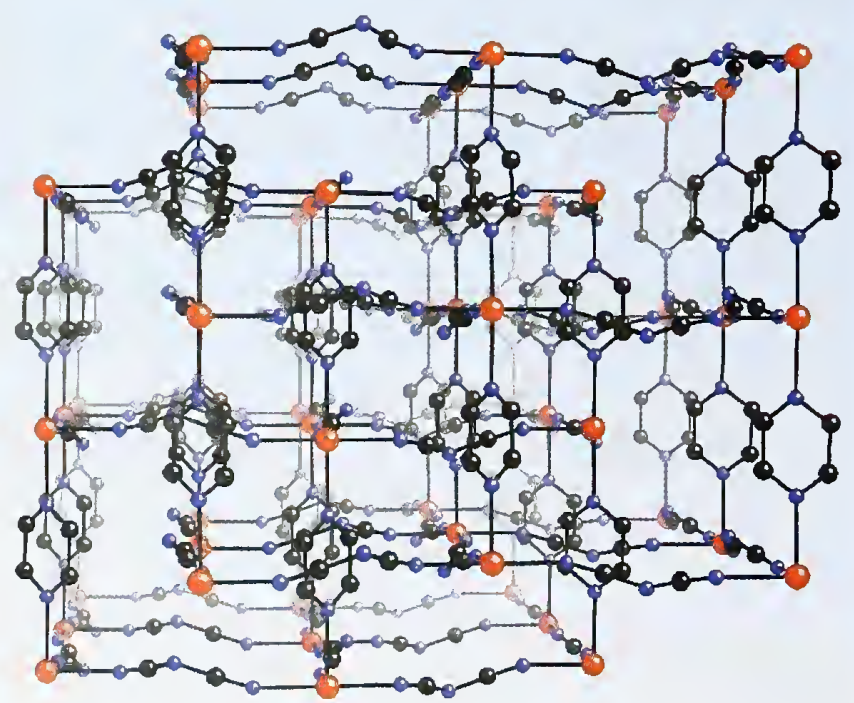

FIGURE 1. Crystal structure of $\mathrm{Mn}\left[\mathrm{N}(\mathrm{CN})_{2}\right]_{2} \cdot$ pyz showing the two independent, interpenetrating $\mathrm{ReO}_{3}$-like lattices. The hydrogen atoms of the pyrazine ring have been omitted, while the $\mathrm{Mn}, \mathbf{C}$, and $\mathrm{N}$ atoms are depicted as red, black, and blue spheres, respectively. neutral pyrazine ligands (Fig. 1) [1]. Upon heating above $\approx 200$ $\mathrm{K}$, Rietveld refinements of neutron powder diffraction (NPD) data indicate a marked increase in the Debye-Waller factor for the midnitrogen in the cyanamide ligand and a concomitant appearance of thermal diffuse scattering. Further heating to $\approx 400 \mathrm{~K}$ results in a phase transition to an unknown structure.

While the structural and magnetic behaviors of these materials have been rather well characterized, very little information has been obtained concerning the interactions that underlie the interesting bonding motifs. Due to its unique sensitivity to hydrogen and the possibility of covering a wide range of timescales, neutron spectroscopy is particularly well suited to probe ligand dynamics that directly reflect the bonding interactions. Quasielastic neutron scattering (QENS) provides information on the geometry and timescale of diffusive motions. For "localized" motions, the geometry is embodied in the elastic incoherent structure factor (EISF); the ratio of the elastic scattering to the total scattered intensity.

For all temperatures studied, a quasielastic signal due to the paramagnetic $\mathrm{Mn}^{2+}$ ions and the dynamics of the cyanamide ligand

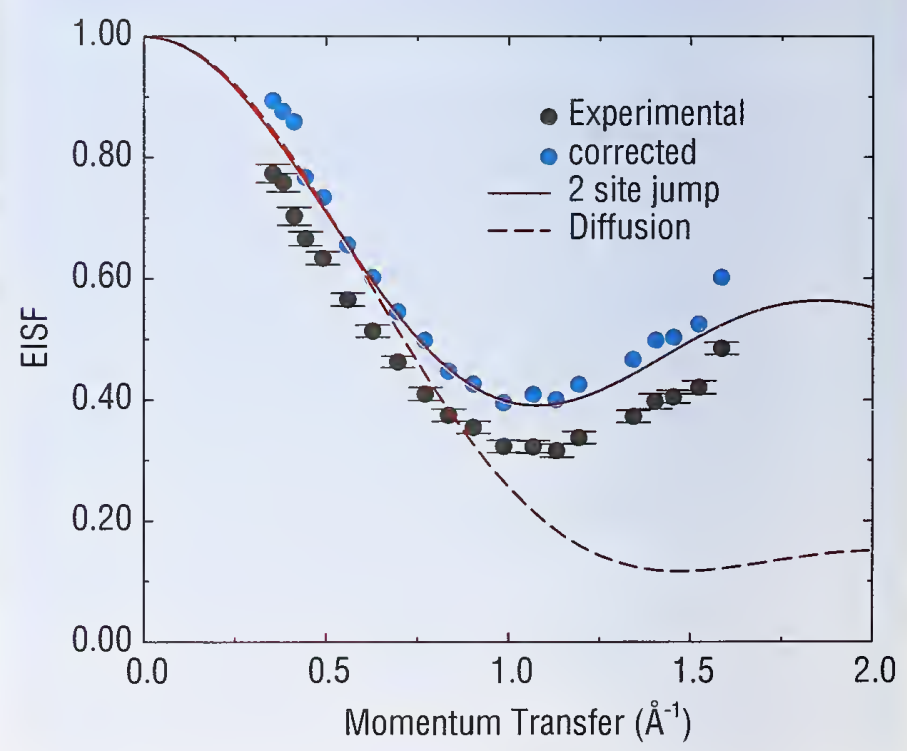

FIGURE 2. The experimental EISF (black circles) for $\mathrm{Mn}\left[\mathrm{N}(\mathrm{CN})_{2}\right]_{2} \cdot \mathrm{pyz}$ at $425 \mathrm{~K}$, has been corrected (blue circles) for coherent and paramagnetic quasielastic scattering. The solid red line is a fit to the corrected data with a 2-fold jump model that results in a proton jump distance of $4.17(1) \AA$. For comparison, the expected EISF for proton diffusion on a circle is also shown (red dashed line). 


\section{M. Brown}

University Of Maryland

College Park, MD 20742 and

NIST Center for Neutron Research

National Institute of Standards and Technology Gaithersburg, MD 20899-8562

\section{J. L. Manson}

Material Science Division

Argonne National Laboratory

Argonne, IL 60439

\section{A. Neumann}

NIST Center for Neytron Research

Nationał Institute of Standards and Technology

Gaithersburg, MD 20899-8562
J. W. Lynn

NIST Center for Neutron Research

National Institute of Standards and Technology Gaithersburg, MD 20899-8562
J. S. Miller

Department of Chemistry

University of Utah

Salt Lake City, UT 84112 was observed. However, above $\approx 425 \mathrm{~K}$, the quasielastic signal in the pyrazine compound is significantly larger. Figure 2 shows the $Q$-dependence of the EISF for the protonated compound and the same data after subtracting the measured quasielastic scattering from a deuterated material. These data are very well described by a twofold proton jump model where the only variable parameter, the jump distance, is found to be 4.17 (1) $\AA$, consistent with the analysis of the NPD data which gives the D-D distance across the pyrazine ring as $\approx 4.16(2) \AA$. Further, the width of the quasielastic peak is constant over the entire $Q$-range, as expected for a simple, localized jump motion (the correlation time is $\approx 70 \mathrm{ps}$ at $425 \mathrm{~K}$ ). Thus, the pyrazine ligands must be performing $\pi$-jumps about the axis defined by the coordinating nitrogens.

The phonon density of states for $\mathrm{Mn}\left[\mathrm{N}(\mathrm{CN})_{2}\right]_{2}$.pyz at selected temperatures covering all structural phases is shown in Fig. 3. In addition to the general broadening and softening of the spectral features with temperature, the intense peak assigned to the libration of the pyrazine ring at $\approx 11.2 \mathrm{meV}$ is strongly attenuated and, assuming a two-fold cosine potential, suggests an activation energy

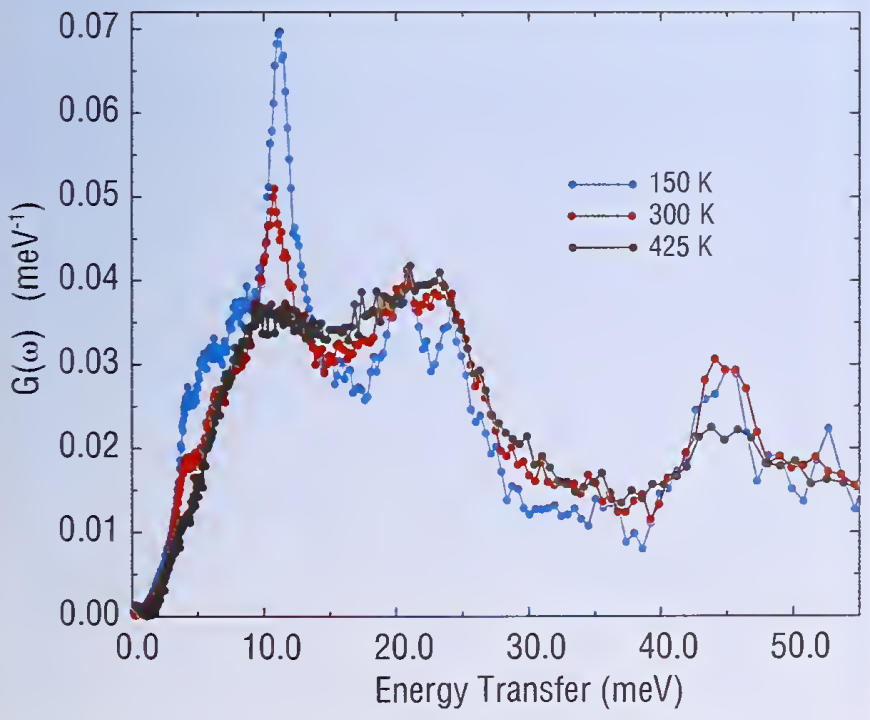

FIGURE 3. Temperature dependence of the vibrational density of states of $\mathrm{Mn}\left[\mathrm{H}(\mathrm{CN})_{2}\right]_{2} \cdot$ pyz measured on the FCS spectrometer at a wavelength of $4.8 \AA$. of $\approx 1.2 \mathrm{eV}$. Because this activation energy is much larger than $\mathrm{k}_{\mathrm{B}} T$ at the temperature where one observes fully dynamic pyrazines ( 425 $\mathrm{K}$ ) and the quasielastic scattering appears abruptly at the structural transition, the transition must result in a greatly reduced rotational barrier. Unfortunately, the activation energy for this process was not measurable due the proximity of the material's decomposition temperature.

Vibrational spectra at higher energy transfers were recorded on the FANS spectrometer (Fig. 4). Our calculations agree well with observation and show that the spectrum is dominated by the normal modes of the hydrogen containing pyrazine. Current investigations are aimed at understanding how the transition metal affects the dynamics of the ligands.

\section{REFERENCES}

[1] J. L. Manson, Q.-Z. Huang, J. W. Lynn, H.-J. Koo, M.-H. Whangbo, R. Bateman, T. Otsuka, N. Wada, D. N. Argyriou, and J. S. Miller. Submitted to Journal of the American Chemical Society.

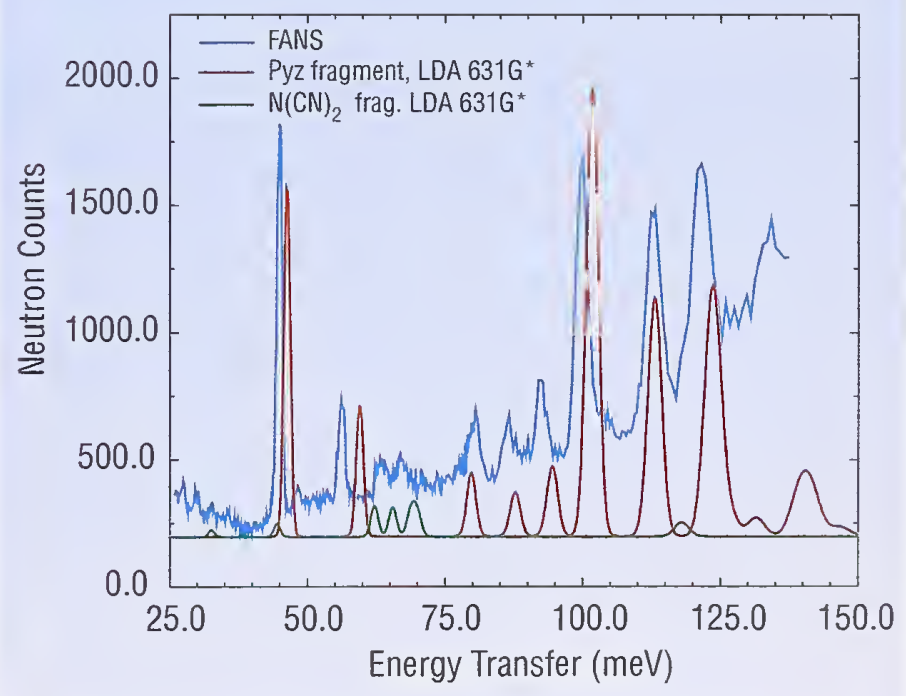

FIGURE 4. The vibrational spectrum (blue) of $\mathrm{Mn}\left[\mathrm{N}(\mathrm{CN})_{2}\right]_{2} \cdot \mathrm{pyz}$ (10 K) measured on the upgraded FANS spectrometer. Shown in red and green are the calculated contributions from chemically simplified, isolated fragments of [Ca-pyz-Ca] ${ }^{4+}$ and [Ca-NCNCN-Ca] ${ }^{3+}$, respectively. 
$T^{* n+n}$ he presence of structural disorder in many materials can have a dramatic effect on macroscopic properties. Typically, structural disorder is described in terms of molecular spatial distributions determined from diffraction measurements. A complementary view is to quantify disorder by determining the distribution of potential energies experienced by the molecules. For a highly ordered system the distribution is sharp while it is broad for a disordered system.

To explore this approach, high-resolution neutron inelastic scattering has been used to examine the low-energy rotational dynamics of simple symmetric top molecules confined to extremely tiny pores. In effect, the porous substrate furnishes a static impurity distributed throughout the molecular solid that can be thought of as disorder quenched into the molecular matrix. The modification to the rotational spectrum of the confined molecules measured using neutron scattering can then be related to the distribution of potential energies. This measurement technique, known as rotational tunneling spectroscopy [1], is extremely sensitive to the environment experienced by the molecules. By modeling the observed spectra one can quantitatively extract the potential energy distribution.

Rotational tunneling spectroscopy is rooted firmly in quantum mechanics. In a simple picture the potential barrier to reorientation

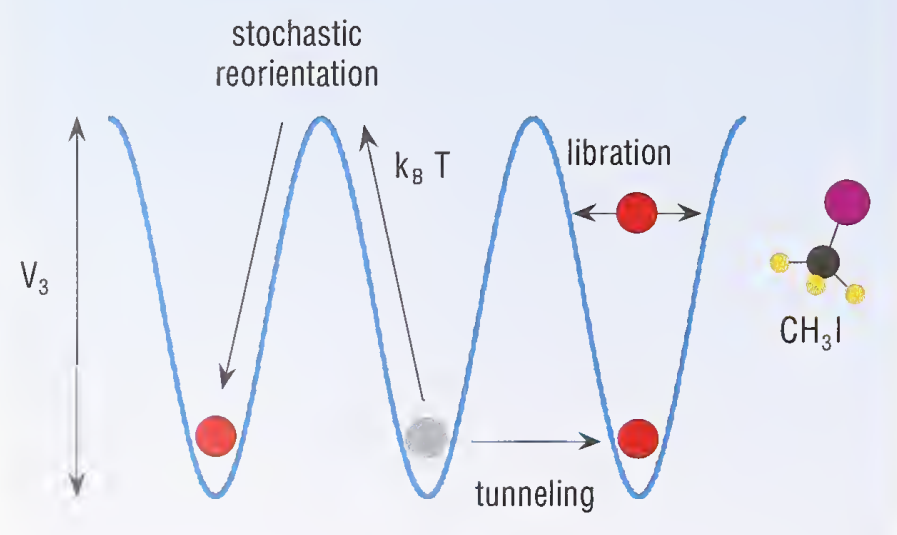

FICURE 1. Potential energy model for a $\mathrm{CH}_{3}$ rotor showing the three types of low energy motions observable with neutrons. Note that each circle located in a potential minimum represents one of three particular orientations of the $\mathrm{CH}_{3}$ methyl group of the $\mathrm{CH}_{3}$ I molecule depicted on the right. that, to a good approximation, has three minima determines the motion of a methyl group (a pyramid with a base of three hydrogen atoms having a carbon atom at its apex seen in Fig. 1). In the limit of a small barrier to reorientation, the methyl group can undergo nearly free rotation about the C-I axis. In the limit of a very high barrier, the methyl group can oscillate (librate) within minima. When the temperature is high enough to provide sufficient kinetic energy, the molecule can reorient stochastically by jumping over the barrier, a process known as rotational diffusion. Some molecules such as methyl iodide $\left(\mathrm{CH}_{3} \mathrm{I}\right)$ possess finite barriers in which the rules of quantum mechanics allow the methyl group to reorient via tunneling through the barrier. A schematic illustration of these processes in terms of the potential energy is shown in Fig. 1.

Measurements of the rotational tunneling of methyl iodide $\left(\mathrm{CH}_{3} \mathrm{I}\right)$ confined to a porous glass with a very narrow pore size distribution (diam $\approx 58 \AA$ ) were performed using the NIST backscattering spectrometer very well suited to such measurements due to its excellent energy resolution: $\delta E(\mathrm{FWHM})<1 \mu \mathrm{eV}$. Measurements were carried out for the bulk solid, partially filled pores, and completely filled pores. Spectra taken at $5 \mathrm{~K}$ are shown in Fig. 2.

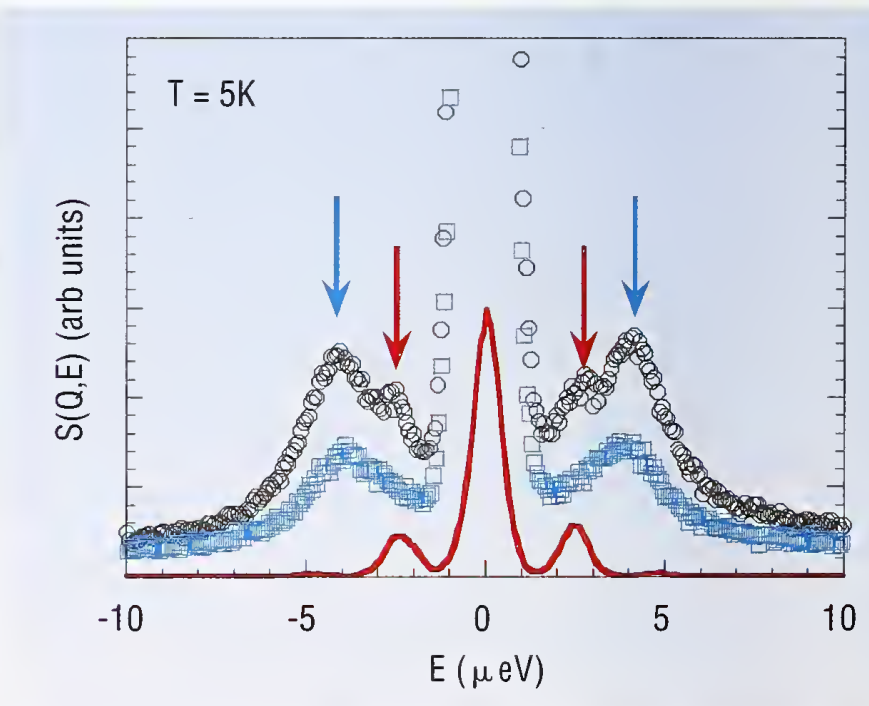

FIGURE 2. Tunneling spectra for the bulk $\mathrm{CH}_{3} \mathrm{I}$ (solid red line), partially filled pores (blue squares), and completely filled pores (black circles). Bulk-like peaks (red arrows) indicate strong order while the broad peaks (blue arrows) indicate that disorder is present. 


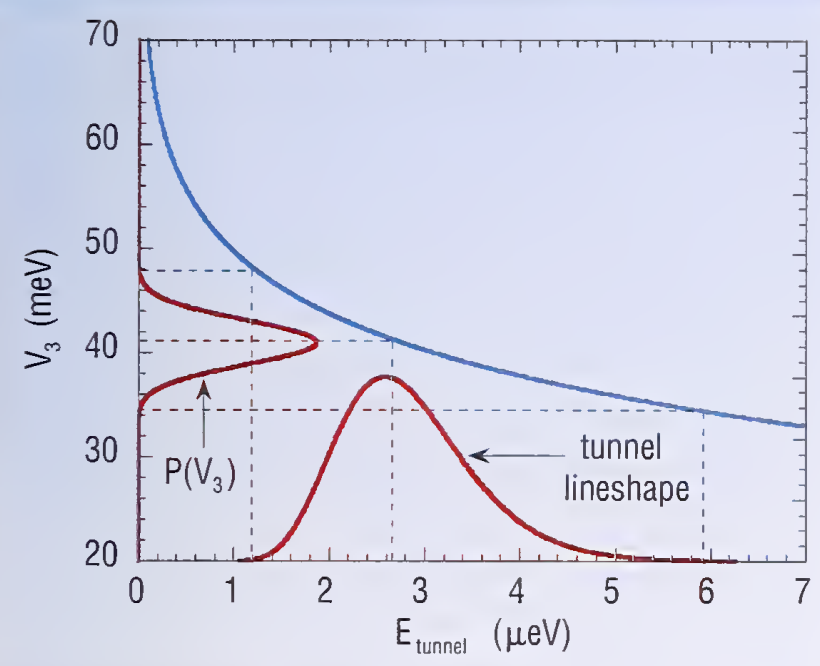

FIGURE 3. Origin of the asymmetric lineshape observed in confined $\mathrm{CH}_{3} \mathrm{I}$. A symmetric distribution of potential barriers yields an asymmetric tunneling lineshape.

The bulk $\mathrm{CH}_{3} \mathrm{I}$ spectrum shows two sharp side peaks whose positions are related to the frequency at which the methyl group $\left(\mathrm{CH}_{3}\right)$ tunnels between three equivalent orientations. The location of these peaks is directly related to the potential barrier height hindering reorientation. These peaks at $\pm 2.5 \mu \mathrm{eV}$ correspond to a potential barrier of $42 \mathrm{meV}$. When the pores are partially filled (50\%) a broad set of peaks appear at $\pm 4 \mu \mathrm{eV}$. In addition to these broad peaks, a broad featureless scattering intensity appears underneath the welldefined peaks over the entire dynamic range. When the pores are completely filled the broad peaks at $\pm 4 \mu \mathrm{eV}$ increase in intensity and a new set of peaks at $\pm 2.5 \mu \mathrm{eV}$ appear. We interpret these different peaks as due to the presence of varying amounts of order in the molecular structure of the confined $\mathrm{CH}_{3} \mathrm{I}$. The narrow peaks that occur in the bulk and the full-pore spectra point to the similarity of the potentials experienced by the molecules as expected when there is structural order. The broad peaks at $\pm 4 \mu \mathrm{eV}$ correspond to methyl groups under the influence of a distribution of potential barriers. The very broad scattering feature underlying both the full-pore and partially-filled samples is attributed to very strongly disordered methyl groups. Based on the filling dependence of the two broad scattering components, these are attributed to molecules near the

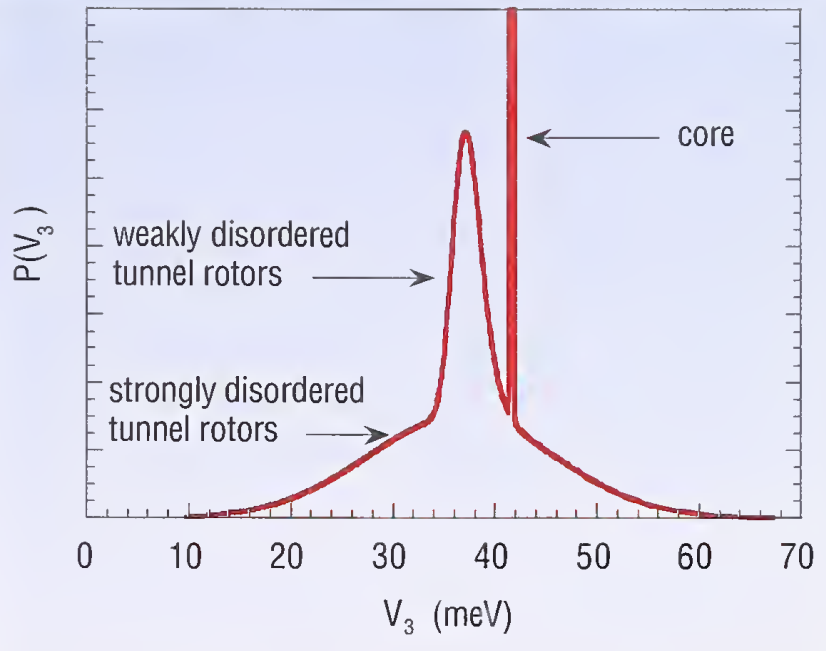

FIGURE 4. The barrier height probability density extracted from full pore tunneling spectra at $5 \mathrm{~K}$.

glass surface while the narrow peaks at $\pm 2.5 \mu \mathrm{eV}$ are due to molecules located near the center of the pore.

To quantify the disorder, we performed numerical calculations of the effects of a distribution of potential barriers on the tunneling lineshape. In Fig. 3 we plot the variation of the potential barrier as a function of tunneling energy. For a broad but symmetric distribution of barrier heights, the tunneling lineshape is clearly asymmetric. Finally, using a relationship between the tunneling energy and barrier height we may extract the probability density for a particular barrier height, $P\left(V_{3}\right)$. The result for the full pore spectra corrected for instrumental resolution is shown in Fig. 4.

Thus neutron inelastic scattering measurements of the rotational tunneling spectrum offer a means of quantifying the disorder of the energy landscape in this system of molecules in a confined geometry. A further challenge is to correlate the energy and structural descriptions of disorder.

\section{REFERENCES}

[1] W. Press, Single-Particle Rotations in Molecular Crystals, Springer-Verlag, Berlin, Heidelberg, New York, (1981). 


\section{PRESSURE-INDUCED INTERLINKING OF CARBON NANOTUBES: COMPUTATIONS}

C arbon nanotubes, originally discovered as byproducts of fullerene synthesis, are now considered to be the building blocks of future nanoscale electronic and mechanical devices. It is therefore desirable to have a good understanding of their electronic and mechanical properties and the interrelations between them. In particular, single wall carbon nanotubes (SWNT) provide a system where the electronic properties can be controlled by the structure of the nanotubes and by various deformations of their geometries $[1,2]$. The physical properties can also be altered by intertube interactions between nanotubes packed in hexagonal lattices, as so-called "nanoropes."

The intertube interactions in nanoropes can be probed by applying external pressure to vary the intertube distance. For fullerenes, such high pressure studies have yielded many interesting results including new compounds such as the pressure-induced polymeric phases of $\mathrm{C}_{60}$. It is, therefore, of interest to inquire if similar covalent-bonding can occur between the nanotubes in a rope. This could have important consequences for nanoscale device applications and composite materials that require strong mechanical properties since nanoropes consisting of inter-linked SWNT will be significantly stronger than nanoropes composed of van der Waals ( $\mathrm{vdW}$ ) packed nanotubes.

We investigated possible new pressure-induced ground state structures for $(\mathrm{n}, 0)$ nanotube ropes from first-principles total energy calculations using the pseudopotential method within the generalized gradient approximation (GGA) [1]. For simplicity, we model the nanoropes as a hexagonal lattice of nanotubes with one nanotube per unit cell. The pressure dependence of the lattices of nanotubes was determined by calculating the total energy as a function of nanotube separation (i.e., a and b) while the other parameters, including atom positions, $\mathrm{c}$, and $\gamma$ are optimized. We observe that $(7,0)$ nanotubes become elliptically distorted with applied pressure (i.e., decreasing nanotube-nanotube distance). At a critical pressure, we observe a structural phase transformation from the $\mathrm{vdW}$ nanotube lattice (as shown in Fig. 1a) to a new lattice in which the nanotubes are interlinked along the [110] direction, where the strain of the nanotube is largest (Fig. 1b). The covalent bonding between nanotubes is therefore the result of curvature-induced re-hybridization of the carbon orbitals. The same structural transformation was observed for the other $(\mathrm{n}, 0)$ nanoropes.

To quantitatively study the bonding mechanism, we calculated the total energies of the different phases as a function of the lattice constant (i.e., applied pressure). The result for $(7,0)$ nanotubes is summarized in Fig. 2. The energies of the vdW and the one-dimensional interlinked phases cross each other at about a $=9.0 \AA$ with an energy barrier of only $46 \mathrm{meV} /$ unitcell $(552 \mathrm{~K})$. The pressure required to attain this lattice constant is only about $0.3 \mathrm{GPa}$ for the $\mathrm{vdW}$ phase, indicating that polymerization of $\mathrm{vdW}(7,0)$ nano-
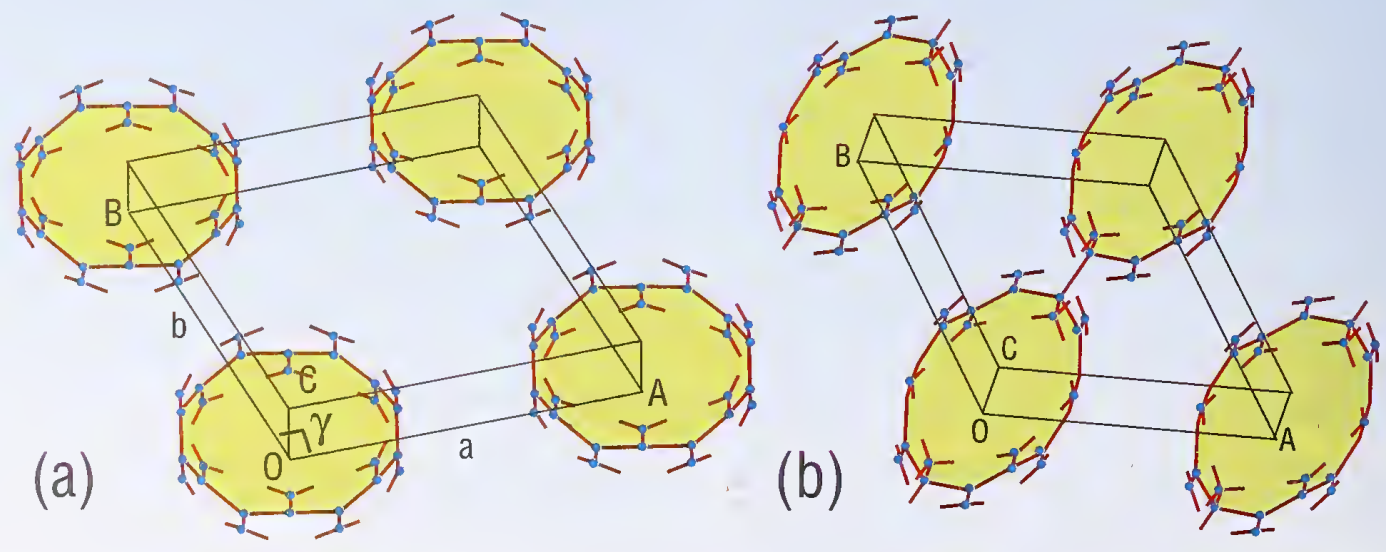

FIGURE 1. Optimized structures of the vdW $(7,0)(a)$, and one- dimensional interlinked $(7,0)$ (b) nanotube lattices. The interlinked structure shown in (b) has lower energy than vdW packed $(7,0)$ nanotubes shown in (a). 


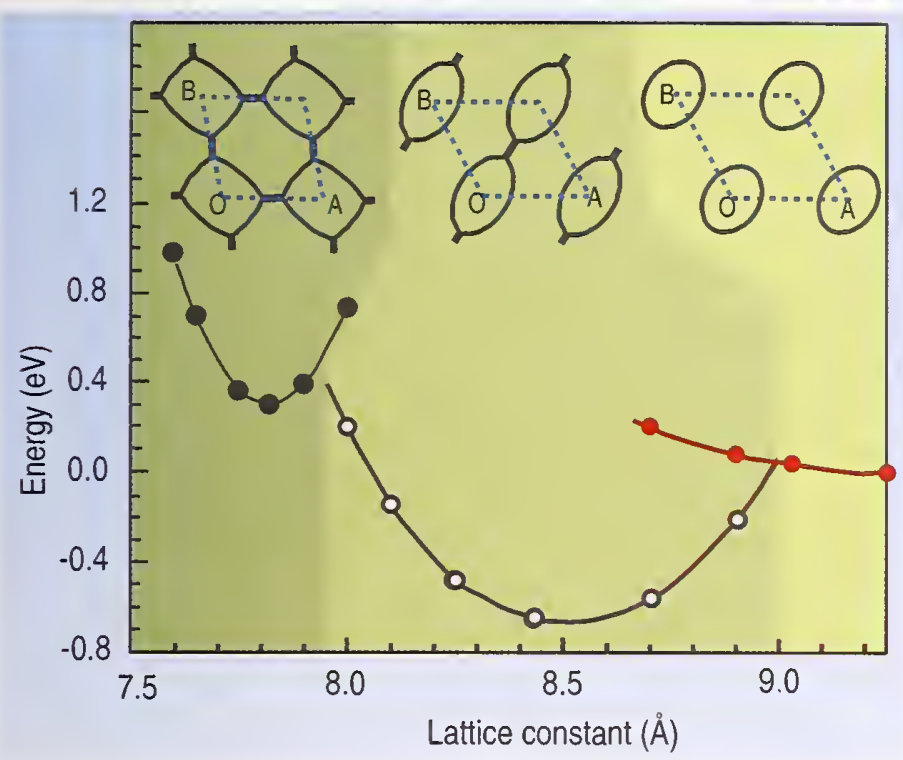

FIGURE 2. Planer lattice constant variation of the total energy of $(7,0)$ nanotube ropes in three different phases. Inset shows the view of the structures along $c$-axis. The zero of energy was taken to be the energy of vdW packing of the nanotubes.

ropes could occur at modest pressures and temperatures. Once the interlinked phase is reached, the energy barrier required to break the bonds and obtain free nanotubes is about $0.7 \mathrm{eV}$ ( $25 \mathrm{meV} / \mathrm{atom}$ ), which is comparable to that of $1 \mathrm{D}$ polymerized $\mathrm{C}_{60}$ molecules (20 meV/atom).

Figure 2 also shows that another interlinked phase of $(7,0)$ nanotubes becomes the ground state for lattice parameter smaller than $8.0 \AA$. In this new phase the nanotubes are interlinked along both a- and b-axes (see Fig. 3a). This 2-D interlinked structure is about four times stiffer than the 1-D interlinked phase and sixteen times stiffer than the vdW nanoropes.

We observe that applying even higher pressures yields more complicated and denser phases for many of the nanoropes studied here (see Fig. 3). For $(9,0)$ nanoropes, we find that the nanotubes are interlinked along three directions forming a hexagonal network. The length of the intertube bond, $\mathrm{d}_{\mathrm{C}-\mathrm{C}}=1.644 \AA$, is significantly elongated for a $\mathrm{sp}^{3} \mathrm{C}-\mathrm{C}$ bond. The two dimensional interlinked phase of $(7,0)$ nanotubes is further transformed to a denser structure at $30 \mathrm{Gpa}$ with a band gap of $2 \mathrm{eV}$ (Fig. 3c). By comparison, $(6,6)$ nanotubes do not form an interlinked structure up to a pressure of $60 \mathrm{GPa}$. Rather the nanotubes are hexagonally distorted such that the local structure of the nanotube faces is similar to that in
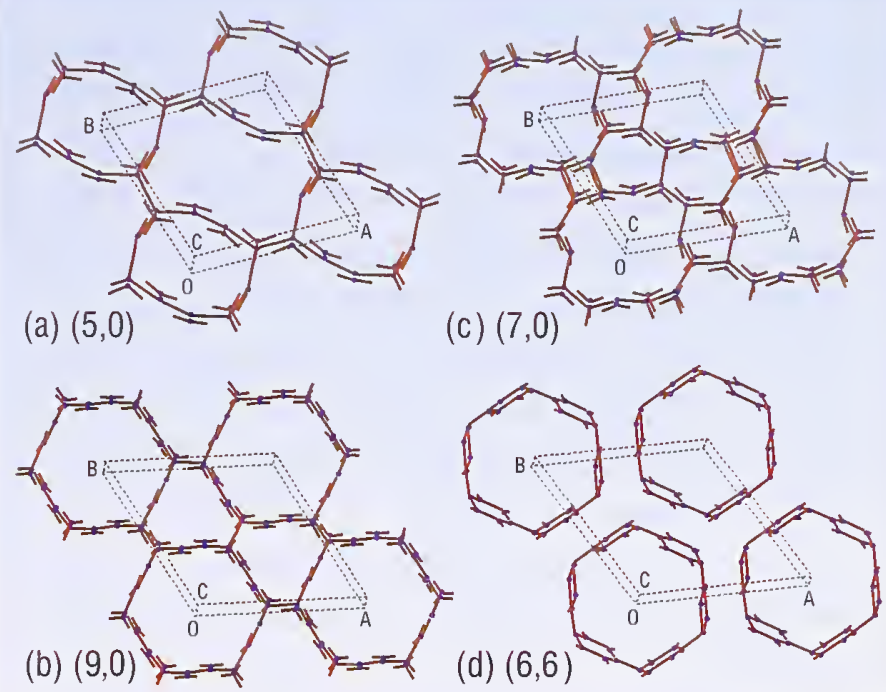

FIGURE 3. Various high density phases of carbon nanotubes. (a) Twodimensional interlinked structure of $(5,0)$ nanotubes, consisting of rectangularly distorted nanotubes interlinked on a 2-D network. (b) A hexagonal network of $(9,0)$ nanotubes, (c) A very dense structure of $(7,0)$ nanotubes obtained under $30 \mathrm{GPa}$ pressure. (d) The optimized structure of $(6,6)$ nanotubes under $P=53$ GPa.

graphite sheets (Fig. 3d). Furthermore, releasing the pressure yields the original structure, indicating that the distortion is purely elastic. The structural changes clearly have strong effects on the electronic properties [2] and therefore should be detected in the pressure dependence of various transport properties of nanoropes.

The new pressure-induced, high density phases [1] reported here may provide a way of synthesizing novel carbon base materials with interesting physical properties. For example interlinking of the nanotubes may improve the mechanical performance of composites based on these materials. The change in the band gap of a SWNT with applied pressure can be exploited to realize various quantum devices on a single nanotube with variable and reversible electronic properties [2]. It will be an experimental challenge to confirm the structures predicted here. A difference-INS spectrum of two identical samples, one treated with pressure and the other not, may give some evidence for the new phases.

\section{REFERENCES}

[1] T. Yildirim, O. Gulseren, C. Kilic, and S. Ciraci, Phys. Rev, B 62, 12648 (2000).

[2] C. Kilic, S. Ciraci, O. Gulseren, and T. Yildirim, Phys. Rev. B 62, RI6345 (2000). 


\section{DYNAMICS CHANGES IN THE \\ MOLTEN GLOBULE-NATIVE FOLDING STEP}

$\mathrm{T}$ he function of a protein depends critically on its ability to adopt a specific structure. Remarkably, a protein can fold efficiently to this native state from the many unfolded states on physiological time scales. Understanding how this process occurs is one of the great challenges in biology. Proteins can also form partially folded, collapsed states under both equilibrium and non-equilibrium conditions [1,2]. Such partially folded proteins resemble the intermediate states along the protein folding pathway, and thus play an important role in understanding the mechanisms of protein folding. Moreover these partially folded proteins have also been shown to participate in important cell functions.

A molten globule is a compact, partially folded protein that has native-like secondary structure and backbone folding topology, but lacks the extensive, specific side-chain packing interactions of the native structure [3]. Structural studies have shown that the sidechains in a molten globule can adopt a greater variety of conformations than in a native protein. The ability of the side chains to form the tight and specific interactions typical of a native protein, is the essential final step in the protein folding pathway. This packing process is considered to be energetically more difficult than forming the collapsed, disordered folding intermediates. However, little is known about the dynamics of this mostly folded state.

In order to understand the changes in protein dynamics that occur in the final stages of folding, we have used incoherent quasielastic neutron scattering (IQNS) to probe the differences in the dynamics between the native state and the almost completely folded, molten globule state of the protein, bovine $\alpha$-lactalbumin in solution [4]. Because hydrogen scatters neutrons much more strongly than deuterium, the exchangeable protons were deuterated and $\mathrm{D}_{2} \mathrm{O}$ was used as the solvent. The scattering from the protein is then dominated by the non-exchangeable protons in the side-chains. Figure 1 shows the measured scattering function, $S(Q, \omega)$, of bovine $\alpha$-lactalbumin (BLA) and its molten globules (MBLA), as a function of the energy transfer $\hbar \omega$ at a momentum transfer $Q=1.08 \AA^{-1}$.

The broader quasielastic peak of MBLA indicates that the side-chain protons within the molten globules move significantly faster than those in the native protein, which reflects the lack of the specific side chain interactions in MBLA compared to BLA.

In addition to the time scale, IQNS yields information on the geometry of the observed motion through the $Q$-dependence of $S(Q, \omega)$. We have analyzed the $Q$-dependence for BLA and MBLA
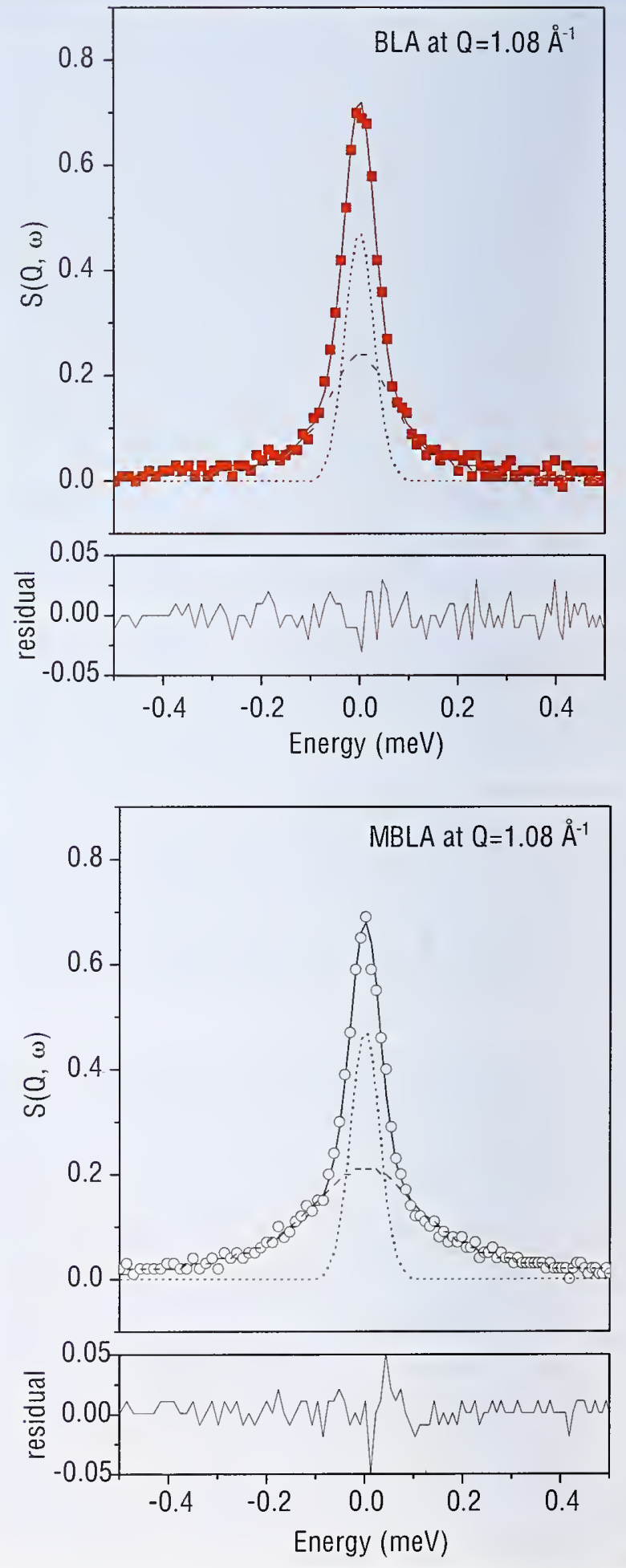

FIGURE 1. The quasielastic neutron scattering spectra, $S(Q, \omega)$ of BLA and MBLA (in arbitrary units), measured as a function of the energy transfer $\hbar \omega$ at $Q=1.08 \AA^{-1}$. The solid lines are fits to the data while the dashed lines show the two components described in the text. 
Dan A. Neumann, Seung-Hun Lee, and Craig M. Brown

NIST Center for Neutron Research

National Institute of Standards and Technology

Gaithersburg, MD 20899-8562
Donald M. Engelman

Department of Molecular Biophysics and Biochemistry Yale University

New Haven, CT 06520-8114 using a model where some of the protons diffuse within a spherical cavity, while others are fixed on the $\approx 70$ ps time scale of these measurements. This is intended to capture the physical picture of side chain motion within a constrained volume imposed by the backbone topology of the protein. Within this model, the scattering consists of two components, a $\delta$-function and a Lorenztian, each broadened with the experimental resolution $\approx 60 \mu \mathrm{eV}$. Typical fits for individual spectra are shown in Fig. 1.

Figure 2 shows the half-width at half-maximum $\Gamma$ of the Lorenztian component of the scattering as a function of $Q^{2}$. The initial linear region indicates that on longer length scales (small $Q$ ), the protons undergo spatially-restricted diffusive motions, while the crossover to a consant width at higher $Q$ reflects the granularity of the motion at these shorter, atomic length scales. The elastic incoherent structure factor, which gives the time-averaged spatial distribution of the protons, is formed by dividing the intenstity of the elastic ( $\delta$-function) component by the total integrated intensity measured at each $Q$. The EISF is shown as a function of $Q$ in Fig. 3. The solid lines show fits to the EISF expected for diffusion within a sphere, showing that the length scale of the motion increases by about $25 \%$ as the side chains become disordered. This is in contrast with the usual situation where slower motions tend to cover larger

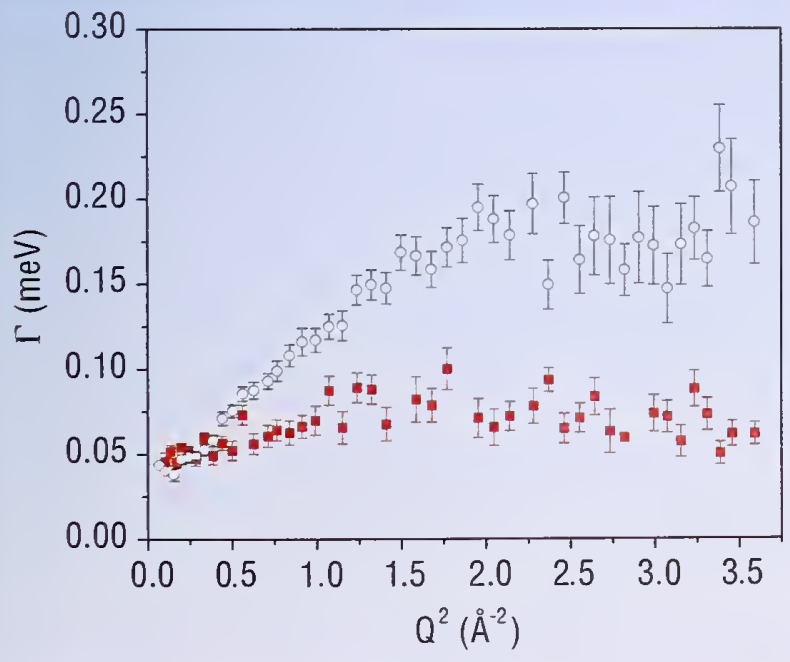

FIGURE 2. The half-width at half-maximum $\Gamma$ of the quasielastic Lorentzian peak as a function of the momentum transfer $Q^{2} ;(\square)$ is for BLA and $(O)$ is for MBLA. The $\Gamma$ vs. $Q$ plot reflects the length dependence of the decay rate of the self-correlation function and is a measure of the mobility of the protons within the protein. length scales. The fact that the EISF plateaus at a higher value for BLA than MBLA indicates that more of the side chain protons are immobilized in the protein's native state.

The mean square amplitude $\left\langle u^{2}\right\rangle$ of the high-frequency vibrational modes can be obtainted from the $Q$-dependence of the total scattering intensity through the Debye-Waller factor. The values of $\left\langle u^{2}\right\rangle$ extracted in this way are indistinguishable for BLA and MBLA, which suggests that chemical bond vibrational motions do not change significantly in the final stage of protein folding.

Overall, these results demonstrate that the side chains in molten globules are significantly more mobile than those in the native protein, and explore a larger length scale in a shorter time. This indicates that the specific side chain interactions responsible for the final step in protein folding both localize and slow the motions of the side chains.

\section{REFERENCES}

[1] K. A. Dill, Biochemistry 29, 7133 (1990).

[2] D. Shortle, FASEB J. 10, 27 (1996).

[3] O. B. Ptitsyn, Adv. Protein. Chem. 47, 83 (1995).

[4] Z. M. Bu, D. A. Neumann, S.-H. Lee, C. M. Brown, D. M. Engelman, and C. C. Han, J. Mol. Biol. 301, $525(2000)$

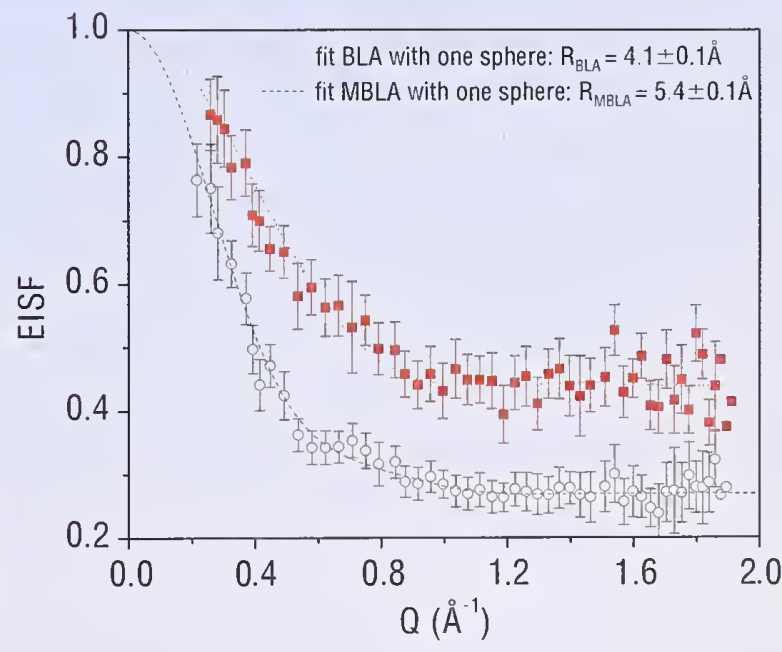

FIGURE 3. The elastic incoherent structure factor (EISF) of ( $\square$ ) BLA and (O) MBLA. The lines are fits to the EISF expected for diffusion within a sphere. The fits show that the length scale of the motion in MBLA is about $25 \%$ larger than in BLA. 


\section{SOFT PHONON ANOMALIES IN RELAXOR FERROELECTRICS}

O ur current phonon studies of ferroelectrics at the NCNR are part of a systematic investigation of $\mathrm{ABO}_{3}$ perovskite oxides that exhibit exceptionally high piezoelectric responses. Two solid solutions, $\left(\mathrm{Pb}\left[\mathrm{Zn}_{1 / 3} \mathrm{Nb}_{2 / 3}\right] \mathrm{O}_{3}\right)_{1-x}\left(\mathrm{PbTiO}_{3}\right)_{x}(\mathrm{PZN}-x \mathrm{PT})$ and $(\mathrm{PbZrO})_{1-x}$ $\left(\mathrm{PbTiO}_{3}\right)_{x}(\mathrm{PZT})$ have been extensively investigated in recent years. A common feature of these two systems is the morphotropic phase boundary (MPB), which separates the tetragonal and rhombohedral regions of the $T-x$ phase diagram. The maximum piezoelectric activity is located on the rhombohedral side of the MPB for both systems. PZN- $x$ PT, however, can sustain ultrahigh strain levels, with $<1 \%$ hysteretic loss, fully one order of magnitude larger than those attainable with conventional PZT-based piezoelectric ceramics. These two remarkable properties suggest that PZN-xPT holds great promise for the next generation of solid-state transducers.

The compositions of the perovskite B-sites of PZN-xPT and PZT differ in a key respect. Whereas an isovalent mixture of $\mathrm{Zr}^{4+}$ and $\mathrm{Ti}^{4+}$ ions occupies the PZT B-site, a more disparate group of heterovalent $\mathrm{Zn}^{2+}$, $\mathrm{Ti}^{4+}$, and $\mathrm{Nb}^{5+}$ ions shares the PZN-xPT B-site. This creates intense quenched random electric fields that are thought to produce the so-called relaxor phase, which is characterized by a diffuse phase transition and a broad and strongly dispersive peak in the dielectric susceptibility. Despite years of research, the physics of this diffuse phase transition is still not well understood. In prototypical ferroelectric systems such as $\mathrm{PbTiO}_{3}$, it is well known that the softening of a zone-center transverse optic (TO) phonon drives the transition from a cubic paraelectric phase to a tetragonal ferroelectric phase. In relaxor compounds such as pure PZN, however, the mixed-valence character of the B-site sharply breaks the translational symmetry, resulting in much more complex lattice dynamics. In fact, no definitive evidence for a soft mode has been found in these relaxor systems.

Motivated by these results, we have examined the lattice dynamics of the polar TO phonon mode in a high quality single crystal of PZN-8 \% PT, for which the measured value of the piezoelectric coefficient $d_{33}$ is a maximum. Figure 1 shows neutron scattering data taken on PZN-8 \% PT in its cubic phase at $500 \mathrm{~K}$ $\left(\approx 50 \mathrm{~K}\right.$ above $T_{\mathrm{c}}$ ) [1]. The maximum scattered neutron intensity has been plotted as a function of energy transfer $\hbar \omega$ and momentum transfer $q$ along the symmetry directions [110] and [001]. The lowest-energy data points trace out a normal TA phonon branch along both [110] and [001]. What is striking, however, is that Fig. 1 shows no evidence of a zone center TO mode at all. Instead, the data suggest a precipitous drop of the TO branch into the TA branch, somewhat resembling a waterfall. This anomalous feature is highlighted by the shaded regions in Fig. 1, and stands in marked contrast to the behavior of $\mathrm{PbTiO}_{3}$ where the same TO phonon branch intercepts the $\hbar \omega$-axis at a finite energy.

To clarify the nature of this unusual observation, we show a constant- $E$ scan at $\hbar \omega=6 \mathrm{meV}$ in Fig. 2 along with a constant $Q$ scan in the insert [1]. Both scans were taken at $500 \mathrm{~K}$ near the (220) Bragg peak, and along the [001] direction. The small horizontal

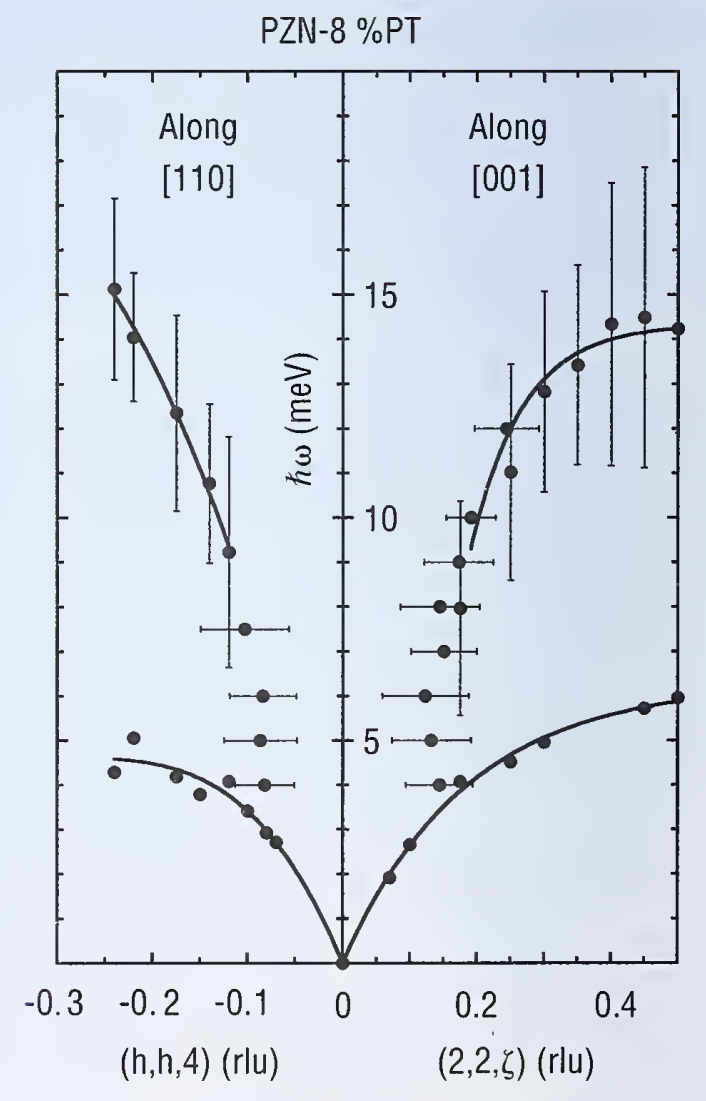

FIGURE 1. Solid dots represent positions of peak scattered neutron intensity taken from constant- $Q$ and constant- $E$ scans at $500 \mathrm{~K}$. Vertical (horizontal) bars represent phonon FWHM linewidths. Solid lines indicate TA and TO phonon dispersions. 


\section{P. M. Gehring}

NIST Center for Neutron Research,

National Institute of Standards and Technology

Gaithersburg, MD 20899-8562

\section{S.-E. Park}

Materials Research Laboratory

The Pennsylvania State University

University Park, PA 16802
G. Shirane

Physics Department

Brookhaven National Laboratory

Upton, NY 11973 bar shown under the left peak of the constant- $E$ scan represents the instrumental FWHM $q$-resolution. We see immediately that the constant- $Q$ scan shows no evidence of any well-defined phonon peak, most likely because the phonons near the zone center are overdamped. However, the constant- $E$ scan indicates the presence of a ridge of scattering intensity at $\zeta=0.13$ r.l.u., or about $0.2 \AA^{-1}$. Thus, the sharp drop in TO branch that appears to take place in Fig. 1 does not correspond to a real dispersion. Rather, it simply indicates a region of $(\hbar \omega, q)$-space in which the phonon scattering cross section is enhanced. The question remains why does this happen?

In 1983 Burns and Dacol proposed a seminal model for the disorder intrinsic to relaxor ferroelectrics [3]. Using measurements

PZN- $8 \%$ PT

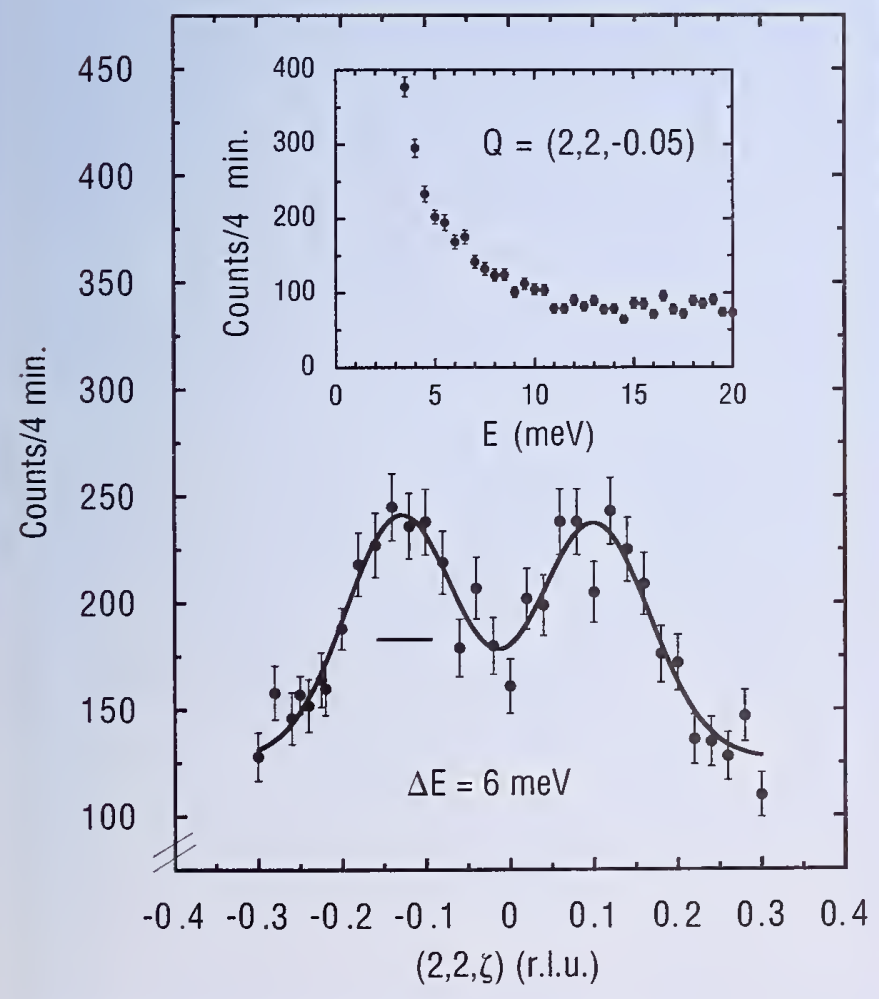

FIGURE 2. Constant- $E$ scan measured along [001] at $6 \mathrm{meV}$ at $500 \mathrm{~K}$ near the (220) Bragg peak. Solid line is a fit to a double Gaussian function of $\zeta$. No peak is discernible in the constant- $Q$ scan shown in the insert. of the optic index of refraction on a variety of samples, including single crystals of PZN, they demonstrated that a randomly oriented local polarization $P_{\mathrm{d}}$ develops at a well-defined temperature $T_{\mathrm{d}}$ several hundred degrees above the transition temperature $T_{\mathrm{c}}$. The origin of this local polarization, not present in normal ferroelectrics above $T_{c}$, was conjectured to arise from the formation of polarized microregions (PMR) of the crystal that are richer in $\mathrm{Nb}^{5+}$ than the average chemical formula.

The presence of such randomly oriented PMR above $T_{\mathrm{c}}$ in PZN-8 \%PT should effectively impede the propagation of polar phonon modes whose wavelength exceeds the size of the PMR. The observation that the phonon scattering cross section is dramatically affected $\approx 0.2 \AA^{-1}$ from the zone center gives a measure of the dynamic size of these polarized domains. If the length scale associated with the anomalous "waterfall" is of order $2 \pi / q$, this would correspond to $31 \AA$, or about 7 to 8 unit cells, a size that is consistent with Burns and Dacol's conjecture. We have recently been able to model this behavior quite well for PZN using a simple coupled-mode that assumes a highly $q$-dependent linewidth $\Gamma(q)$ that increases sharply for $q<0.2 \AA^{-1}$ [2]. Hence we speculate that the striking anomalies in the TO phonon branch shown in Fig. 1 (the same branch that goes soft at the zone center at $T_{\mathrm{c}}$ in $\mathrm{PbTiO}_{3}$ ) for $q<$ $0.2 \AA^{-1}$ are directly caused by these PMR which serve to dampen the zone center TO phonon modes. If true, then this unusual behavior should be observed in other related relaxor systems. Direct evidence for this has already been observed at room temperature in neutron scattering measurements on $\mathrm{P} M \mathrm{~N}(M=\mathrm{Mg})$ [4].

\section{REFERENCES}

[1] P. M. Gehring, S.-E. Park, and G. Shirane, Phys. Rev. Lett. 84, 5216 (2000).

[2] P. M. Gehring, S.-E. Park, and G. Shirane, to be submitted to Phys. Rev. B.

[3] G. Burns and F. H. Dacol, Phys. Rev. B 28, 2527 (1983); ibid. Solid State Commun. $48,853,(1983)$.

[4] P. M. Gehring, S. B. Vakhrushev, and G. Shirane, in Proceedings of the Fundamental Physics of Ferroelectrics 2000 Winter Workshop, Aspen, 2000, edited by R. E. Cohen (American Institute of Physics, 2000), p. 314. 


\section{LITHOSILICATES: A NEW FAMILY OF MICROPOROUS MATERIALS}

henever you have a novel structure you usually get novel
properties." Edith M. Flanigen [1] (First winner of the International Zeolite Association lifetime contribution and achievement award).

Zeolites and related microporous materials contain regularly spaced molecule-sized pores and are fascinating examples of molecular engineering. More than just aesthetically inspiring, as can be seen in Fig. 1, these materials represent billions of dollars in commerce, as they have widespread industrial applications: Materials of this type are used as catalysts to produce gasoline and pharmaceuticals. For medical and industrial purposes, they are employed to separate $\mathrm{N}_{2}, \mathrm{O}_{2}$, and other gases. Formulated in household detergents, they remove the calcium ions that make water "hard," replacing environmentally unfriendly phosphates. Zeolites and related microporous materials are also employed to sequester radioactive ions for bioremediation. Many new applications are being investigated in areas such as selective membranes, batteries, and fuel cells.

Properly used, the term zeolite should be restricted to naturally occurring aluminosilicates. However, related microporous materials (herein called zeolitic) have been synthesized, where elements such as $\mathrm{B}, \mathrm{Be}$, $\mathrm{Cr}, \mathrm{Fe}, \mathrm{Ga}, \mathrm{Ge}, \mathrm{Mn}, \mathrm{P}, \mathrm{Ti}$, and $\mathrm{Zn}$ substitute for tetrahedrally bonded Si or Al. These atoms have roughly the same bonding configurations, so they tend to adopt the same framework connectivities as aluminosilicates and thus have the same pore structures. Further, since each tetrahedral $(T)$ atom is linked by four oxygen atoms, the frame-

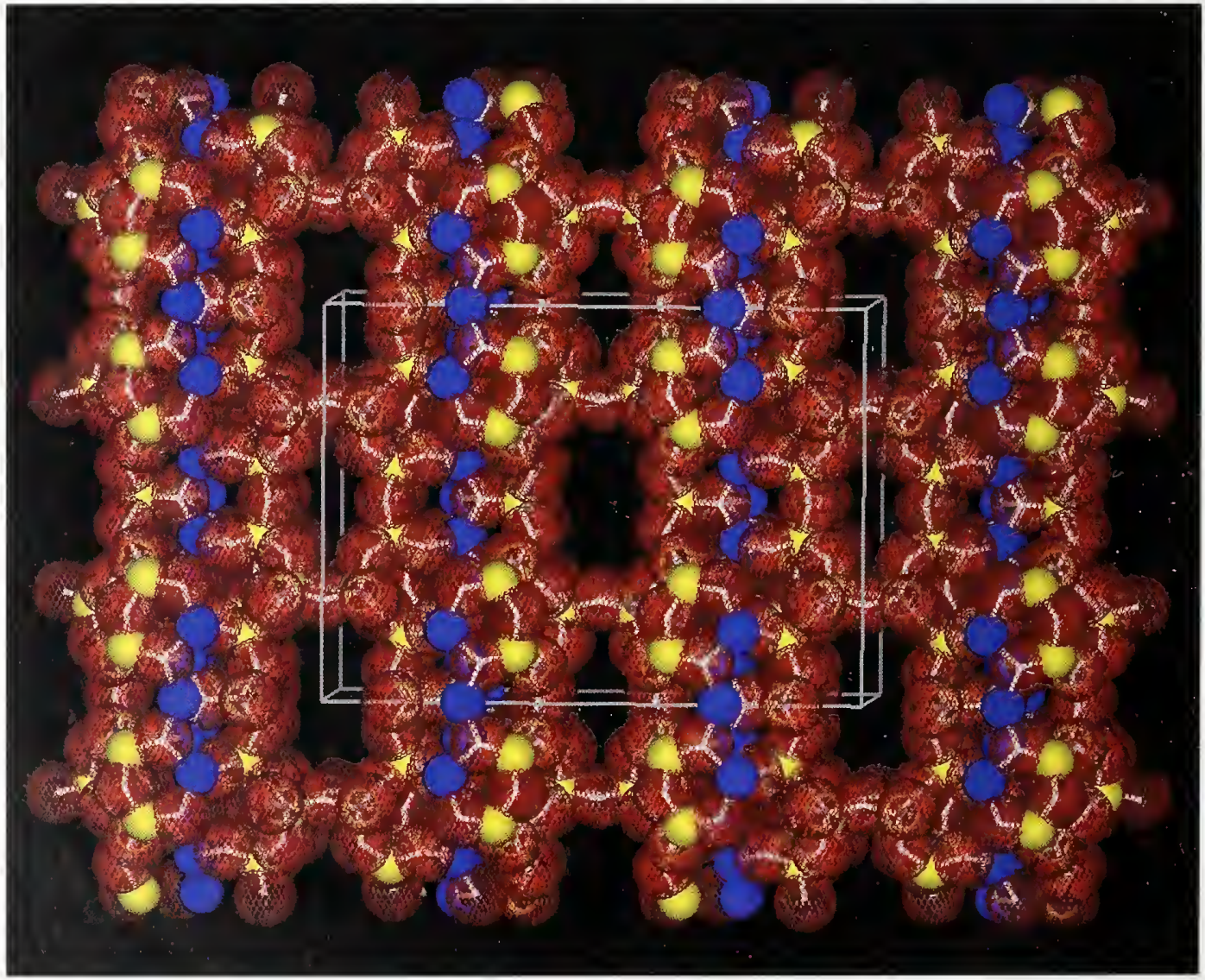

FIGURE 1. The RUB-29 framework. Tetrahedral Si and Li atoms are shown as solid yellow and blue spheres, respectively at their approximate covalent radius (1 $\AA$ ). Framework $\mathbf{0}$ atoms are shown as transparent red spheres at their approximate Van der Waals radius (1.4 Å). Extra-framework species are omitted for clarity. work must have a generalized chemical formula $\left[T \mathrm{O}_{2}\right]_{1 \cdot x}{ }^{m}\left[T^{\prime} \mathrm{O}_{2}\right]_{x}{ }^{n}$ where the charge on each tetrahedral unit ( $m$ or $n$ ) is typically zero or -1. Additional extra-framework cations, such as $\mathrm{H}^{+}, \mathrm{Na}^{+}, \mathrm{Li}^{+}$, or $\mathrm{Ca}^{2+}$, are then needed to balance changes. The number and nature of these cations is significant as cations are often the chemically active site for interactions with guest species, or their presence may affect the framework properties. Thus, they may dictate the catalytic or sorptive properties of the material. Empirically, the most negatively charged zeolitic frameworks that have been found, e.g., those that have the largest number of extra-framework cations, are materials with formula $\mathrm{M}^{+}\left[\mathrm{SiO}_{2}\right]\left[\mathrm{AlO}_{2}\right]^{-1}$. Pauling's rules, restated in the zeolite field as Lowenstein's rule, dicate that the ratio of $\mathrm{Al}$ :Si cannot exceed unity, limiting the cation fraction to $1 / 7$ the total number of atoms.

A new class of materials that have Li incorporated as a tetrahedral framework species has recently been discovered [2,3]. 


\section{So-Hyun Park}

Department of Geosciences

State University of New York at Stony Brook

Stony Brook NY 11790-2100

and

NIST Center for Neutron Research

National Institute of Standards and Technology

Gaithersburg, MD 20899-8562
John B. Parise, Haiming Liu, and Clare P. Grey

Chemistry Department and Department of Geosciences

State University of New York at Stony Brook

Stony Brook, NY 11790-2100

Hermann Gies

Institut für Geologie, Mineralogie, Geophysik

Ruhr-Universität-Bochum

Bochum, D-44801, Germany
Brian H. Toby

NIST Center for Neutron Research

National Institute of Standards and Technology

Gaithersburg, MD 20899-8562
These materials, called microporous lithosilicates, are novel for two reasons. One is that $\left[\mathrm{LiO}_{4}\right]$ tetrahedra appear more flexible than other $\left[\mathrm{TO}_{4}\right]$ tetrahedra. This means that lithosilicates can bond in configurations that are too strained to exist for other silicates. Thus, lithosilicates offer the promise of new families of pore structures. Second, the general formula for these materials is $M_{3 . x}+\left[\mathrm{SiO}_{2}\right]_{1-x}\left[\mathrm{LiO}_{2}\right]_{x}^{3-}$, so that lithosilicates have the potential to be more negatively charged than aluminosilicates. if the Li:Si ratio exceeds 1:4.

A team of NCNR scientists and collaborators has recently completed the first complete structural characterization of a microporous lithosilicate, RUB-29. To determine the framework geometry, synchrotron diffraction measurements were performed at the NSLS using an extraordinarily small single crystal - with dimensions 10 $\mu \mathrm{m} \times 10 \mu \mathrm{m} \times 2 \mu \mathrm{m}$. (For comparison, human hair is typically $50 \mu \mathrm{m}$ to $100 \mu \mathrm{m}$ thick.) With 35 symmetry-unique atoms comprising the framework, RUB-29 is one of the most complex zeolitic structures. Powder neutron diffraction at NIST was then used to better determine the siting of the framework Li atoms, as well as four additional extra-framework Li atoms and seven other extraframework species. The RUB-29 framework is shown in Fig. 1.

The structural studies of RUB-29 demonstrate two novel structural building blocks, a Li,Si-spiro-3,5 and a Li,Si-spiro-5 unit (see Fig. 2). It should be noted that both these building units contain "three-rings" where three $T$ atoms, in this case one $\mathrm{Li}$ and two $\mathrm{Si}$ atoms, are bonded in a cyclic structure. This three-ring structural entity is highly strained in silicates; only one silicate example has ever been found.

In RUB-29, only 1 in $5 T$ atoms are $\mathrm{Li}$, so the total framework charge is comparable to 1:1 aluminosilicates. However, RUB-29 appears to be stable under conditions where these high-aluminum zeolites tend to degrade. Further, there is promise that new lithosilicate materials can be synthesized with even higher Li:Si ratios.

Another interesting property exhibited by RUB-29 is that the Li atoms, both in framework and non-framework sites, appear to move on an NMR timescale at temperatures as low as $250{ }^{\circ} \mathrm{C}$. Much more work is needed to learn about conduction in this material, but it may hold promise for ionic conduction applications, such as in batteries.

\section{REFERENCES}

[1] S. Borman, R. Dagani, R. L. Rawls, and P. S. Zurer, Chemical \& Engineering News, January 12, 1998.

[2] S.-H. Park, P. Daniels, and H. Gies, Microporous Mater. 37, 129 (2000).

[3] S.-H. Park, et al. Journal of the American Chemical Society (2000), in press.
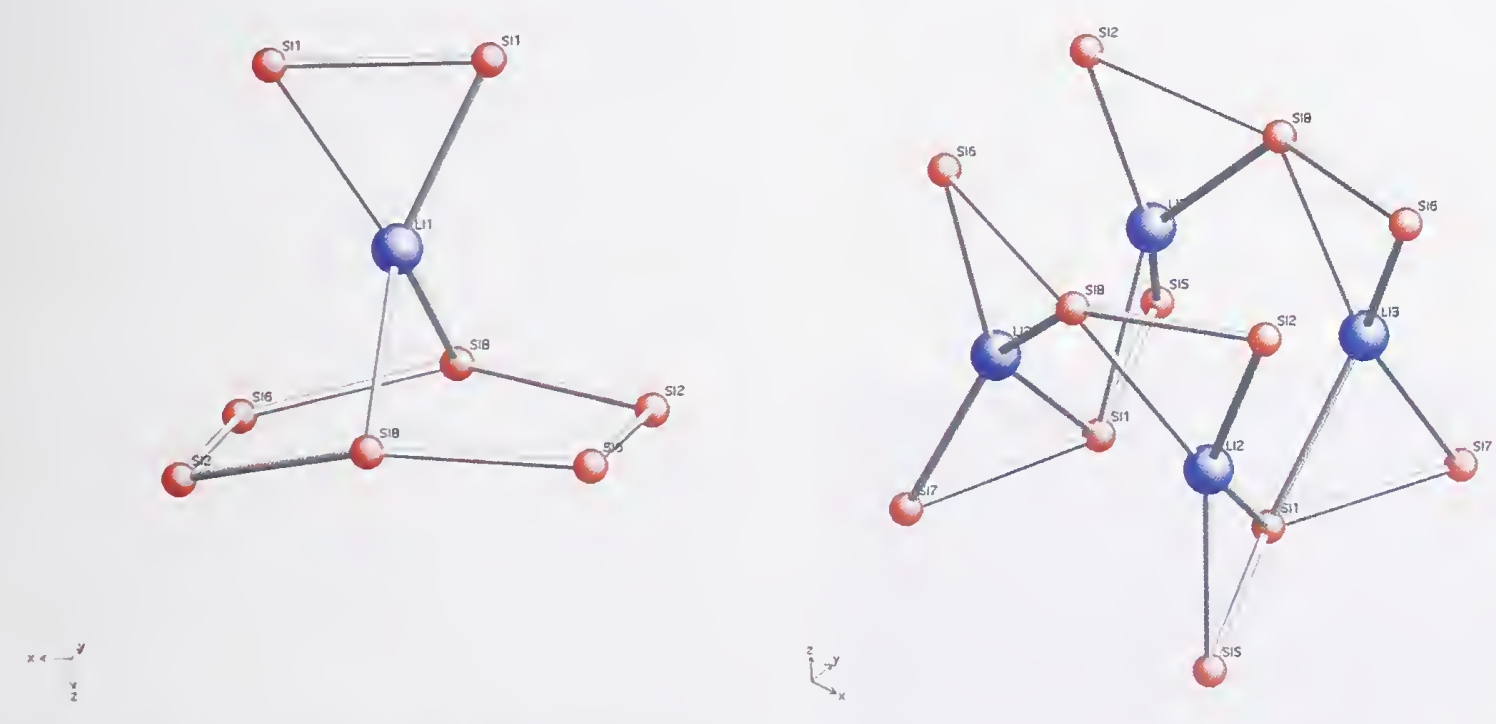

FIGURE 2. Li,Si-spiro-3,5 (left) and Li,Si-spiro-5 (right). Small red circles indicate the centers of [SiO $]_{4}$-tetrahedra, and big blue circles are those of [LiO]-tetrahedra. The 0 atoms that bridge each pair of Si and Li atoms have been omitted to improve clarity. 


\section{QUANTIFICATION OF PHASE FRACTION AND AMORPHOUS CONTENT}

W hile many methods can be used to determine the elemental composition of a material, diffraction is one of the few techniques that is also sensitive to the physical arrangement of atoms and molecules in the solid state. Even phases with the same chemical composition, such as graphite and diamond, have different diffraction patterns, dictated by the particular structure of each substance. The diffraction pattern from a mixture is the weighted sum of the patterns from each phase that is present, making it possible for quantitative phase composition to be determined. Prior to the application of Rietveld refinement techniques to the problem of quantitative phase analysis, these measurements required difficult calibrations and were often imprecise. However, Rietveld analysis involves the fitting of the entire diffraction pattern of each component phase based upon a structural model of the material and no standards or prior calibration is required. It should be noted though that even Rietveld analysis could not be used directly to quantify materials with unknown structures or amorphous phases, since such materials cannot be modeled crystallographically. An example where quantitative phase analysis explains the inevitable failure of ceramic thermal barrier coatings was presented in the 1998 NCNR Annual Report.

NIST participation in a round robin on determination of quantitative phase abundance, sponsored by the International Union of Crystallography Commission on Powder Diffraction, provided an excellent opportunity to demonstrate the high quality of data obtained using the 32-detector NCNR high-resolution powder diffractometer at BT-1. The NCNR implementation of the Rietveld technique for phase quantification was found to give excellent agreement with the nominal compositions. In addition, a new method for determining the amorphous phase content of a mixed-phase sample without sample adulteration was validated.

Data were collected using a $\mathrm{Cu}(311)$ monochromator $(\lambda=1.5402 \AA)$ and 15 ' incident collimation, and were then processed in the usual procedure to obtain a pseudo-single detector data set. The phase fractions were determined using standard Rietveld refinement techniques, including full refinement of crystallographic and instrumental parameters, as implemented by the GSAS suite of programs. Results for a sample consisting of nearly equal mass fractions of $\mathrm{Al}_{2} \mathrm{O}_{3}, \mathrm{CaF}_{2}$, and $\mathrm{ZnO}$, along with values reported by the other round-robin participants, are given in Table 1; the results obtained at NIST for all other samples are reported in Table 2 along with the nominal phase content. It can be seen from Table
Table 1. Mass fractions (\%) of three-phase mixture reported by round robin participants. The ranges are reported for $111 \mathrm{x}$-ray determinations, 7 neutron determinations, and 4 synchrotron determinations. Standard uncertainties are reported in parentheses for the XRF ( $x$-ray fluorescence, 3 determinations as reported by the organizers) and NIST results (determined from the least-squares refinement).

$\begin{array}{lcccccc}\text { Phase } & \text { Weighed } & \text { XRF } & \text { x-ray } & \text { Neutron } & \text { Synchrotron } & \text { NIST } \\ \mathrm{Al}_{2} \mathrm{O}_{3} & 31.4 & 31.7(1) & 22-49 & 29-32 & 31-35 & 31.7(1) \\ \mathrm{CaF}_{2} & 34.4 & 33.9(1) & 19-42 & 32-39 & 34-35 & 33.9(1) \\ \mathrm{ZnO} & 34.2 & 34.0(1) & 25-42 & 32-35 & 29-35 & 34.4(1)\end{array}$

1 that neutron data give significantly more accurate results than synchrotron or laboratory $\mathrm{x}$-ray data, and that the results obtained at NIST are exceedingly good. The high accuracy of these results can be attributed to the intrinsic Gaussian line shape of the reactor neutron source, as well at to the lack of microabsorption and preferred orientation effects that frequently plague $\mathrm{x}$-ray data but are normally negligible with neutron data. The data presented in Table 2 further confirm this conclusion, in that the results for sample 2 (preferred orientation), sample 3 (amorphous content) and sample 4 (microabsorption) all agree well with the nominal phase content.

The determination of amorphous content in a crystalline sample has traditionally involved integration of the area under the broad amorphous hump, giving the relative intensity compared with that of the Bragg scattering. However, in multiphase samples this technique is impractical. An alternative approach is to add a known quantity of a material as an internal intensity standard.

The unique properties of neutron diffraction suggested an alternative approach. Since absorption is negligible for most elements, and since the entire sample is irradiated in the neutron beam, a strategy based on absolute scattering intensities using an external standard was devised.

In the Rietveld technique, the mass of each crystalline phase, $w_{\mathrm{i}}$, is proportional to the product of the scale factor for that phase, $S_{\mathrm{i}}$, and the molecular weight of the unit cell contents $\left(Z_{\mathrm{i}} M_{\mathrm{i}}\right)$ where $Z_{\mathrm{i}}$ is the number of formula units per unit cell and $M_{\mathrm{i}}$ is the molecular weight. Thus for a crystalline multiphase sample of mass $w_{\mathrm{C}}\left(w_{\mathrm{C}}=\right.$ $\sum w_{\mathrm{j}}$ ) the relation $w_{\mathrm{C}} \propto \sum S_{\mathrm{i}} Z_{\mathrm{i}} M_{\mathrm{i}}$ is true. The proportionality constant can be determined using mass $w_{\text {std }}$ of a completely crystalline known standard under identical data collection conditions, so the relation $w_{\mathrm{C}} / w_{\text {std }}=\left(\sum S_{\mathrm{i}} Z_{\mathrm{i}} M_{\mathrm{i}}\right) /\left(S_{\mathrm{std}} Z_{\mathrm{std}} M_{\mathrm{std}}\right)$ can be used. If the sample mass, $w_{\mathrm{S}}$, 
Table 2. Comparison of nominal phase composition (\%) with that obtained at NIST. The weighed and XRF values were provided by the round robin organizers. Standard uncertainties for the NIST results are given in parentheses.

\begin{tabular}{|c|c|c|c|c|}
\hline Sample & Component & $\begin{array}{l}\text { Mass fraction } \\
\text { weighed }\end{array}$ & $\begin{array}{c}\text { Mass fraction } \\
\text { XRF }\end{array}$ & $\begin{array}{l}\text { Mass fraction } \\
\text { NIST }\end{array}$ \\
\hline $1 a$ & $\begin{array}{l}\mathrm{Al}_{2} \mathrm{O}_{3} \\
\mathrm{CaF}_{2} \\
\mathrm{ZnO}\end{array}$ & $\begin{array}{r}1.2 \\
94.8 \\
4.0\end{array}$ & $\begin{array}{r}1.2 \\
94.1 \\
4.1\end{array}$ & $\begin{array}{r}1.3(1) \\
94.8(3) \\
3.9(1)\end{array}$ \\
\hline $1 b$ & $\begin{array}{l}\mathrm{Al}_{2} \mathrm{O}_{3} \\
\mathrm{CaF}_{2} \\
\mathrm{ZnO}\end{array}$ & $\begin{array}{r}94.3 \\
4.3 \\
1.4\end{array}$ & $\begin{array}{r}94.7 \\
4.3 \\
1.4\end{array}$ & $\begin{array}{r}94.4(3) \\
4.2(1) \\
1.4(1)\end{array}$ \\
\hline $1 c$ & $\begin{array}{l}\mathrm{Al}_{2} \mathrm{O}_{3} \\
\mathrm{CaF}_{2} \\
\mathrm{ZnO}\end{array}$ & $\begin{array}{r}5.0 \\
1.4 \\
93.6\end{array}$ & $\begin{array}{r}5.1 \\
1.3 \\
93.2\end{array}$ & $\begin{array}{r}4.9(1) \\
1.4(1) \\
93.8(3)\end{array}$ \\
\hline $1 d$ & $\begin{array}{l}\mathrm{Al}_{2} \mathrm{O}_{3} \\
\mathrm{CaF}_{2} \\
\mathrm{ZnO}\end{array}$ & $\begin{array}{l}13.5 \\
53.6 \\
32.9\end{array}$ & $\begin{array}{l}13.8 \\
53.0 \\
33.0\end{array}$ & $\begin{array}{l}13.7(1) \\
53.0(2) \\
33.3(1)\end{array}$ \\
\hline $1 e$ & $\begin{array}{l}\mathrm{Al}_{2} \mathrm{O}_{3} \\
\mathrm{CaF}_{2} \\
\mathrm{ZnO}\end{array}$ & $\begin{array}{l}55.1 \\
29.6 \\
15.3\end{array}$ & $\begin{array}{l}55.8 \\
29.4 \\
15.3\end{array}$ & $\begin{array}{l}55.4(2) \\
29.2(1) \\
15.4(1)\end{array}$ \\
\hline $1 f$ & $\begin{array}{l}\mathrm{Al}_{2} \mathrm{O}_{3} \\
\mathrm{CaF}_{2} \\
\mathrm{ZnO}\end{array}$ & $\begin{array}{l}27.1 \\
17.7 \\
55.2\end{array}$ & $\begin{array}{l}27.3 \\
17.4 \\
54.9\end{array}$ & $\begin{array}{l}26.7(1) \\
17.5(1) \\
55.8(2)\end{array}$ \\
\hline 2 & $\begin{array}{l}\mathrm{Al}_{2} \mathrm{O}_{3} \\
\mathrm{CaF}_{2} \\
\mathrm{ZnO} \\
\mathrm{Mg}(\mathrm{OH})_{2}\end{array}$ & $\begin{array}{l}21.3 \\
22.5 \\
19.9 \\
36.3\end{array}$ & $\begin{array}{l}20.9 \\
24.1 \\
19.5 \\
36.1\end{array}$ & $\begin{array}{l}21.9(4) \\
22.0(4) \\
19.1(6) \\
37.0(3)\end{array}$ \\
\hline 3 & $\begin{array}{l}\text { Amorphous } \\
\mathrm{Al}_{2} \mathrm{O}_{3} \\
\mathrm{CaF}_{2} \\
\mathrm{ZnO}\end{array}$ & $\begin{array}{l}29.5 \\
30.8 \\
20.1 \\
19.7\end{array}$ & $\begin{array}{l}27.1 \\
31.1 \\
19.9 \\
19.6\end{array}$ & $\begin{array}{l}32.3(6) \\
30.7(6) \\
18.7(3) \\
18.3(3)\end{array}$ \\
\hline 4 & $\begin{array}{l}\mathrm{Al}_{2} \mathrm{O}_{3} \\
\mathrm{Fe}_{3} \mathrm{O}_{4} \\
\mathrm{ZrSiO}_{4}\end{array}$ & $\begin{array}{l}50.5 \\
19.6 \\
29.9\end{array}$ & $\begin{array}{l}50.4 \\
19.6 \\
29.5\end{array}$ & $\begin{array}{l}50.5(3) \\
20.3(2) \\
29.3(4)\end{array}$ \\
\hline
\end{tabular}

also contains an amorphous component, $w_{\mathrm{A}}$, then since $w_{\mathrm{A}}=w_{\mathrm{S}}-w_{\mathrm{C}}$ and the amorphous fraction $f_{\mathrm{A}}=w_{\mathrm{A}} / w_{\mathrm{S}}$, then

$$
f_{\mathrm{A}}=1-\frac{\sum S_{\mathrm{i}} Z_{\mathrm{i}} M_{\mathrm{i}}}{S_{\text {std }} Z_{\text {std }} M_{\text {std }}} \cdot \frac{w_{\text {std }}}{w_{\mathrm{S}}} .
$$

Using the GSAS suite of programs, the values for $S_{\mathrm{i}}$ may be obtained from the product of the overall scale factor and the "phase fraction," or by fixing the overall scale factor to 1.0 so that the refined "phase fractions" are in fact the Rietveld scale factors for each phase.

In order to make sure that data on the unknown and standard materials were collected under identical conditions the automated sample changer was used, the sample volume was reduced to ensure complete sample irradiation, and identical data collection parameters were employed. In this way, data on any number of unknowns could be compared to a single standard sample since no changes were made to the experimental conditions. An additional benefit was that no adulteration or mixing of the samples was necessary. This technique was used with several single-phase samples in order to compare the crystallinity of potential standards as well as of the unknown samples. In fact, the round robin organizers subsequently sent samples of each of the unmixed phases to NIST for analysis. The results obtained for the round robin sample with amorphous content gave excellent agreement with the mass fractions determined by weighing (see Table 2); the slightly higher amorphous content obtained using the neutron Rietveld technique is explained by the presence of a small amount ( $1 \%$ to $2 \%$ ) of amorphous material in the component crystalline phases.

This external standard technique to determine amorphous content could also be used to determine the mass fraction of a crystalline phase with an unknown structure. For both applications, however, obtaining the best results depends upon obtaining the best diffraction data. The unique capabilities of the NCNR high-resolution diffractometer at BT-1 make this possible; these are summarized below.

\section{FEATURES OF THE BT-1 DIFFRACTOMETER}

Automatic six-carousel locking sample changer (vanadium sample cans), ideal sample size: $10 \mathrm{~cm}^{3}$.

Maximum beam size: $15 \mathrm{~mm}$ width by $50 \mathrm{~mm}$ height.

Collimations: $15^{\prime}$ or 7' before monochromator, 20' before sample [30' for $\mathrm{Si}(531)], 7^{\prime}$ before detectors.

Detectors: $32^{3} \mathrm{He}$-filled detectors at $5^{\circ}$ intervals; $13^{\circ}$ scan range covering $2 \theta$ from $0^{\circ}$ to $167^{\circ}$.

Monochromators:

$\begin{array}{lccccccc}\begin{array}{c}\text { Monochromator } \\ \text { crystal and } \\ \text { Bragg plane }\end{array} & \begin{array}{c}\text { In-pile } \\ \text { Collimation } \\ \text { angle (") }\end{array} & \begin{array}{c}\text { Monochromator } \\ 2 \theta\left({ }^{\circ}\right)\end{array} & \begin{array}{c}\text { Relative } \\ \text { Bragg } \\ \text { Intensities }\end{array} & \begin{array}{c}\text { Filux } \\ \text { (neutrons } \mathrm{s}^{-1} \\ \left.\mathrm{~cm}^{-2}\right)\end{array} & \begin{array}{c}\text { Wavelength } \\ (\AA)\end{array} & \begin{array}{c}\text { Typical } \\ \text { run times } \\ \text { (h) }\end{array} \\ \mathrm{Ge}(311) & 15 & 75 & 3.34 & 700000 & 2.0784 & 0.5-5 \\ \mathrm{Ge}(311) & 7 & 75 & 1.84 & 400000 & 2.0795 & \\ \mathrm{Cu}(311) & 15 & 90 & 1.00 & 400000 & 1.5401 & 3-12 \\ \mathrm{Cu}(311) & 7 & 90 & 0.59 & 200000 & 1.5405 & \\ \mathrm{Si}(531) & 15 & 120 & 0.47 & 200000 & 1.5903 & 6-24 \\ \mathrm{Si}(531) & 7 & 120 & 0.33 & 100000 & 1.5904 & \end{array}$




\section{DIFFRACTION ELASTIC CONSTANTS FOR ARBITRARY SPECIMEN AND CRYSTAL SYMMETRIES}

ccurate determination of residual stresses by means of dif-

fraction relies on the knowledge of the elastic constants that translate lattice strain into macro-stress. Because of the difference between the elastic behavior of the aggregate and that of a single crystallite, the straightforward relationship between strain and stress as mediated by single crystal or polycrystal elastic constants no longer holds. Instead, the relationship between lattice strain and macro-stress is mediated by diffraction elastic constants (DEC).

Very recently, we proposed a theory that allows a transparent calculation of DEC. This theory applies for almost the entire range of polycrystalline elasticity, including that for aggregates of arbitrary phase composition and arbitrary symmetry, both of the aggregate

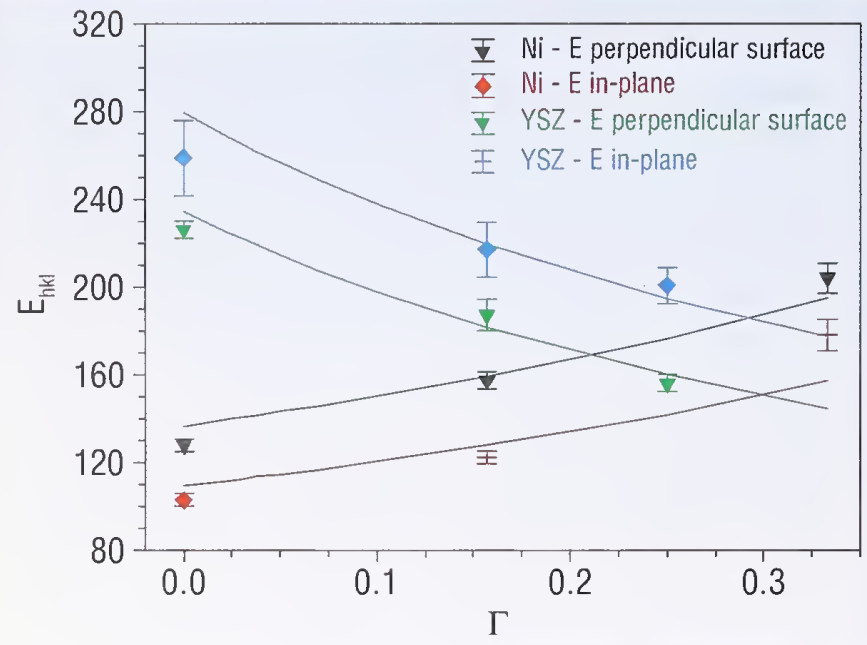

FIGURE 1. Young's modulus vs. orientation parameter for a metallic nickel coating and a ceramic YSZ $\left(\mathrm{ZrO}_{2}+8 \% \mathrm{Y}_{2} \mathrm{O}_{3}\right)$ coating deposited by plasma spraying. Solid lines indicate calculated values, points are measured values. and of the constituents [1]. Results show that for a particular crystallographic plane $(h k l)$ and an arbitrary anisotropic material there are usually six independent DEC. These DEC depend on the orientation of the scattering vector, on the grain shape, and on the elastic constants both of the crystallites and of the aggregate. Figure 1 shows Young's modulus vs. the orientation parameter for different crystallographic planes ( $h k l$ ) of two plasma-sprayed coatings with different types of anisotropy. Calculated DEC for comparison to measurement were not previously available.

Figure 1 also illustrates the difference between the anisotropy of the aggregate and that of the crystallites that comprise the aggregate. The slope of $E_{h k l}$ vs. $\Gamma$ depends mostly on the ratio

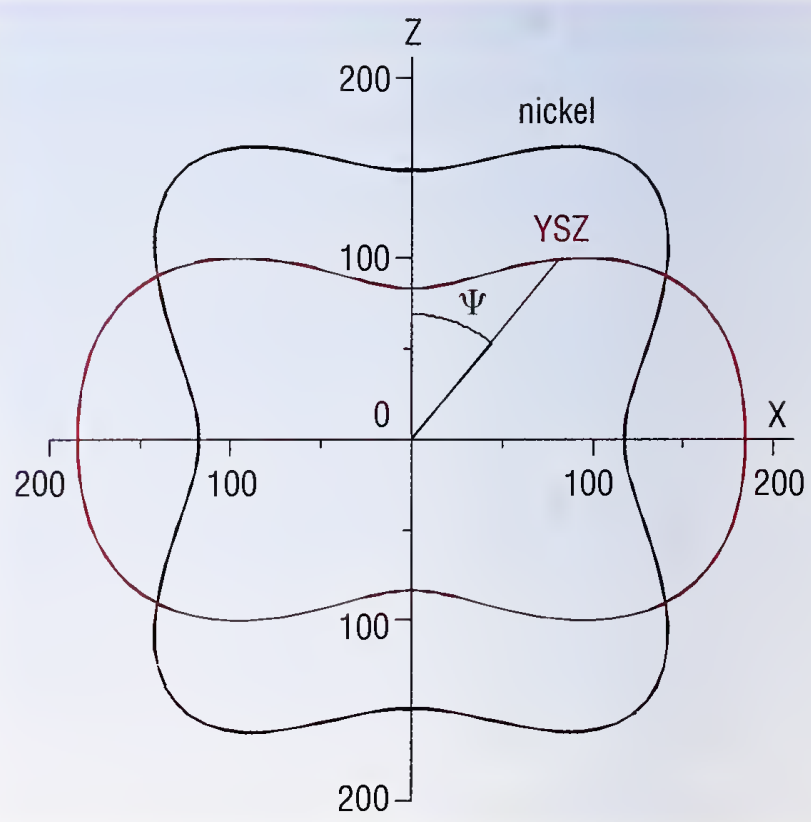

FIGURE 2 . Central section of the representation surface for Young's modulus in a nickel coating and a yttria stabilized zirconia coating. The length of the radius vector for a certain tilt angle $\Psi$ is equal to Young's modulus in units of $\mathrm{GPa}$. These values were calculated from hkl-dependent elastic constants measured by neutron diffraction. The z-direction is perpendicular to the surface of the coating. All in-plane directions are equivalent due to the transversal-isotropic elastic symmetry of sprayed coatings. 
Thomas Gnäupel-Herold

NIST Center for Neutron Research

National Institute of Standards and Technology

Gaithersburg, MD 20899-8562

and

University of Maryland

College Park, MD 20742

\section{H. J. Prask}

NIST Center for Neutron Research

National Institute of Standards and Technology

Gaithersburg, MD 20899-8562
$2 C_{4} /\left(C_{11}-C_{12}\right)$ which is $>1$ for nickel and $<1$ for YSZ. The aggregates exhibit generally transversal isotropic elastic symmetry but again with a different anisotropy in the metallic coating $\left(C_{11} / C_{33}<1\right)$ and in the ceramic coating $\left(C_{11} / C_{33}>1\right)$. This anisotropy causes a substantial difference between $E_{h k l}$ normal and perpendicular to the coating surface.

While the data in Fig. 1 still depend on the crystallographic plane $(h k l)$ they can also be used to estimate the overall elastic constants of the aggregates that, in turn, yield the directional dependence of the mechanical value of Young's modulus. This distribution forms a surface with rotational symmetry around the coating surface normal vector as illustrated by a central section shown in Fig. 2 .
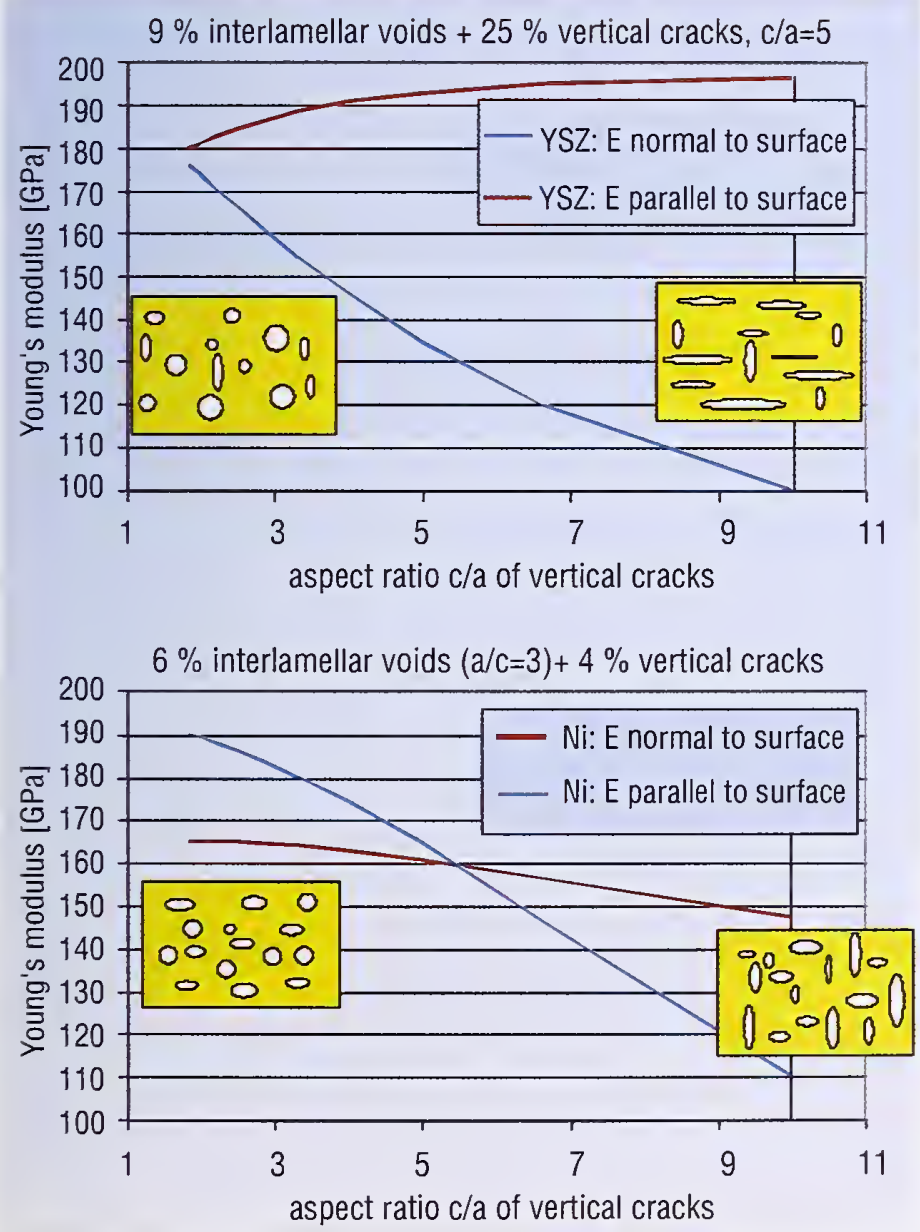

FIGURE 3. Young's modulus for the YSZ coating (a) and for the nickel coating (b) vs. the pore aspect ratio. The vertical lines at aspect ratio of 10 indicate where the best agreement is found with experimental results.
The elastic anisotropy of sprayed coatings has its roots in their microstructure. In the complete absence of preferred crystallite orientation, the responsible factor is the porosity and alignment of elongated voids and cracks resulting from the spray process. These voids can be treated as another phase with very low elastic moduli. This way it is possible estimate the overall elastic constants if the distribution of pore shapes and volume fractions are known.

In the case that the aggregate constants are already known, the reverse approach of estimating a pore distribution can offer some insight into the properties of different coatings. Figs. $3 \mathrm{a}$ and $\mathrm{b}$ show where the best agreement was found for plasma sprayed metallic and ceramic coatings. The main difference is that the pore structure of the ceramic coating is dominated by interlamellar (horizontal) voids, while the concentrations of horizontal voids and vertical cracks are more balanced in the metallic coating.

\section{REFERENCES}

[1]. T. Gnäupel-Herold, J. Matejicek, and H.J. Prask, "Mechanical Properties of Plasma Sprayed Coatings - Measured by Diffraction," to be published in Proc. of the 9th Int. Metallurgical Conf. Metal 2000, Ostrava, Czech Republic, May 16-18, 2000; T. Gnäupel-Herold, and H.J. Prask, "Diffraction Elastic Constants for Arbitrary Specimen and Crystal Symmetries: Theory and Practical Consequences," in Proc. of the 6th Int. Conf. on Residual Stresses (ICRS-6), (IOM Communications Ltd., UK, 2000), pp. 243-250. 


\section{DIRECT OBSERVATION OF SUPERHEATING AND SUPERCOOLING OF VORTEX MATTER}

1 current question of fundamental interest concerns whether a vortex solid-liquid transition exists in type-II superconductors [1]. In addition to providing a possible model system for melting and freezing, vortex matter offers unprecedented opportunities to study the effects of quenched disorder on phase transitions. The peak effect, where the critical current exhibits a peak rather than decreasing monotonically with increasing temperature, has been found to occur at the same temperature as a magnetization jump, which suggests a melting of the vortex lattice. However, there has been no direct structural evidence indicating whether there is indeed an underlying phase transition, and if so, whether it is solid-to-solid, solid-to-liquid, or even liquid-to-liquid in origin. Moreover, since quenched disorder is known to have important consequences for phase transitions, whether a solid-liquid transition can occur when random pinning is effective has broad implications in condensed matter physics.

Here we report the first observation of a striking history dependence of the structure function of vortex matter in the peak effect regime in a Nb single crystal, using SANS combined with simultaneous magnetic susceptibility measurements [2]. Metastable supercooled vortex liquid and superheated vortex solid phases have been observed, providing direct structural evidence for a first-order vortex solid-liquid transition associated with the peak effect.

Measurements were performed on a $\mathrm{Nb}$ single crystal, with the incident neutron beam nominally along the cylindrical axis which coincides with the three-fold symmetric $<111>$ crystallographic direction. A superconducting magnet applies a dc magnetic field along the same direction. The peak-effect regime is determined in situ by measuring the characteristic dip in the temperature dependence of the real-part of the ac magnetic susceptibility $\chi^{\prime}$, as shown in Fig. I(a) for $H=3.75 \mathrm{kOe}$ [2]. The pronounced diamagnetic dip in $\chi^{\prime}(\mathrm{T})$ of the ac susceptibility corresponds to a strong peak effect in the critical current. The onset, the peak, and the end of the peak effect are denoted by $T_{\mathrm{o}}(H), T_{\mathrm{p}}(H)$, and $T_{\mathrm{c} 2}(H)$, respectively. Figure 1(b) shows the window of the experiment.

For each $(T, H)$, we measure the SANS patterns for different thermal paths. At sufficiently low temperatures the SANS images show sharp Bragg peaks with six-fold symmetry, independently of the thermal history. An example is shown in the inset of Fig. l(b) for $H=3.75 \mathrm{kOe}$ and $T=3.50 \mathrm{~K}$. However, the vortex pattern starts to show striking history dependence as the peak-effect regime is approached. We define the field-cooled (FC) state as when the sample is cooled to the target temperature in a magnetic field, while the zero-field-cooled (ZFC) state is reached by cooling the sample in zero field to the target temperature and then increasing the magnetic field to the desired value. For a field-cooled-warming (FC-W) state, the system is cooled in-field to a low temperature $(\approx 2 \mathrm{~K})$, then warmed back to the final temperature.

For the FC path, the vortex patterns show nearly isotropic rings for $T_{\mathrm{p}}<T<T_{\mathrm{c} 2}$ and broad Bragg spots for $T<T_{\mathrm{p}}$. In contrast, for the ZFC and the FC-W paths, sharp Bragg spots are observed for all temperatures up to $T_{\mathrm{c} 2}$. Shown in the top panel of Fig. 2 are
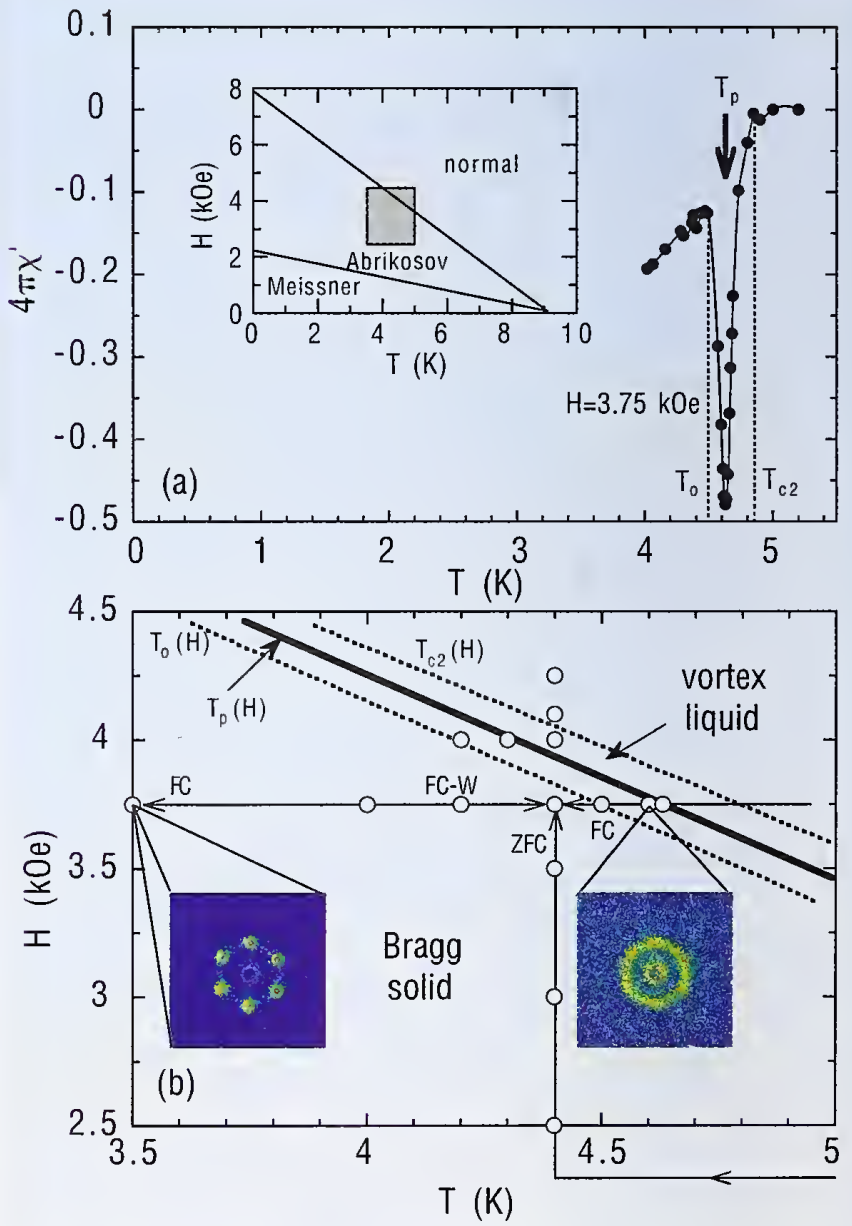

FIGURE 1. Peak effect and $(H-\eta)$ phase diagram of $\mathrm{Nb}$. a) ac magnetic susceptibility for $H_{\mathrm{dc}}=3.75 \mathrm{kOe}$ (field-cooled). $H_{\mathrm{ac}}=3.3 \mathrm{Oe}$ and $1 \mathrm{kHz}$. Inset: global $H-T$ phase diagram for the $\mathrm{Nb}$ crystal used in this study. (b) Expanded view of the $H-T$ phase diagram (shaded box in a). Two observed SANS images of the field-cooled vortex states are shown. 
X. S. Ling, S. R. Park, and

B. A. McClain

Department of Physics

Brown University

Providence, RI 02912

\section{S.-M. Choi}

NIST Center for Neutron Research

National Institute of Standards and Technology

Gaithersburg, MD 20899-8562

and

University of Maryland

College Park, MD 20742
D. C. Dender and J. W. Lynn

NIST Center for Neutron Research

National Institute of Standards and Technology

Gaithersburg, MD 20899-8562 the $\mathrm{ZFC}$ and $\mathrm{FC}$ images at $(3.75 \mathrm{kOe}, 4.40 \mathrm{~K})$, which is just below $T_{0}(3.75 \mathrm{kOe})=4.50 \mathrm{~K}$. The images in the mid panel are for $(4.00$ kOe, $4.40 \mathrm{~K})$, which is $0.10 \mathrm{~K}$ above $T_{\mathrm{p}}(4.0 \mathrm{kOe})=4.30 \mathrm{~K}$. The intensities at the radial maximum for the mid panel SANS data are plotted in the lower panel. The sharp Bragg spots for the ZFC state indicate a vortex lattice with long-range-order (LRO), while the very broad spots for the FC state signify a disordered phase with short-range-order.

The observed hysteresis suggests a first-order vortex solidliquid (or glass) transition. A controversial issue is the location
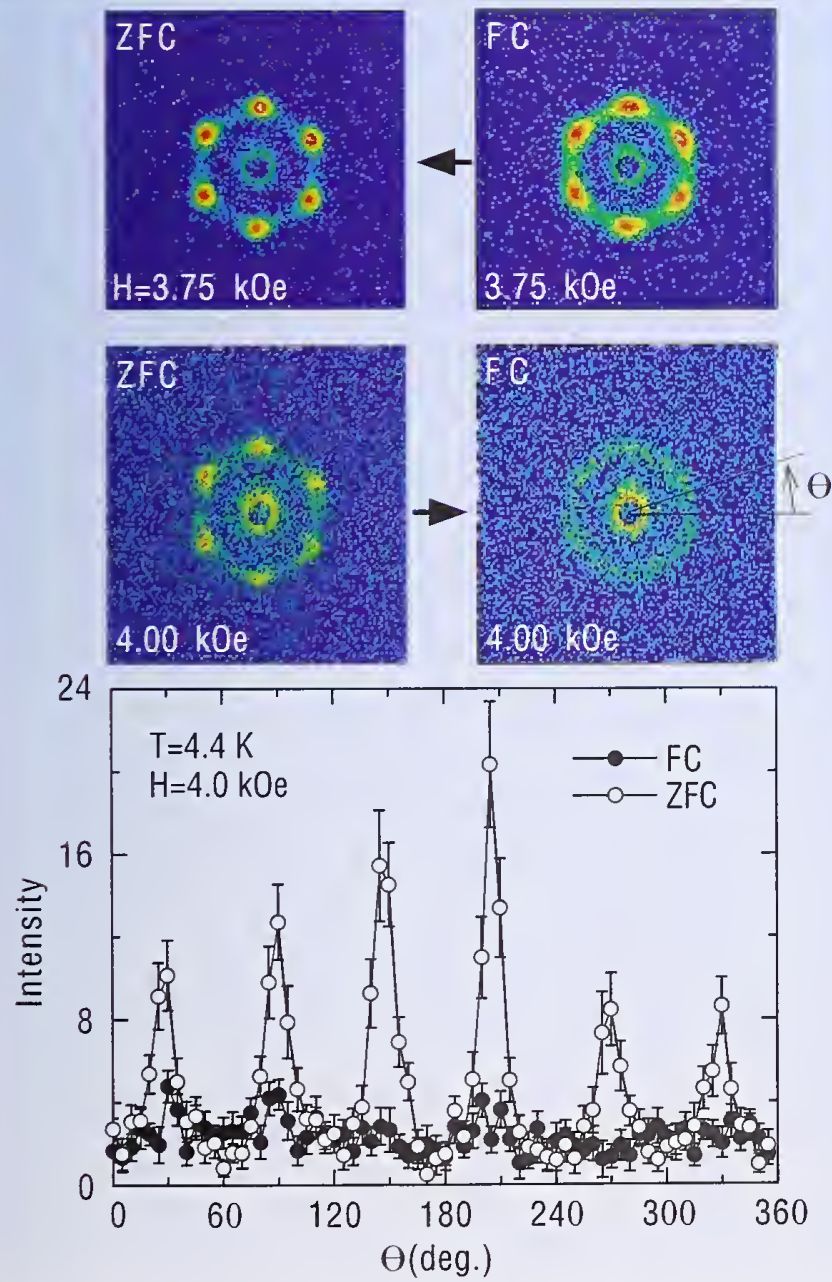

FIGURE 2. History-dependent SANS patterns at $4.40 \mathrm{~K}$. The SANS images of the ZFC and FC vortex states for $\boldsymbol{H}=3.75 \mathrm{kDe}$ (top panel: below the onset of the peak effect) and $\boldsymbol{H}=4.00 \mathrm{kOe}$ (mid panel: near the upper end of the peak-effect regime). The thick arrows indicate how the SANS images evolve after applying a small ac magnetic field. The lower panel shows the intensity data at the radial maximum as a function of the azimuthal angle for the ZFC and FC SANS data $(H=4 \mathrm{kOe})$. of the underlying equilibrium phase transition to the position of the peak effect. One interpretation places the conjectured vortex solid-liquid transition $T_{\mathrm{m}}$ at $T_{\mathrm{p}}$, consistent with the recent experiments in YBCO. Another widely held view is based on the classical Lindemann criterion which would place $T_{\mathrm{m}}$ at $T_{\mathrm{c} 2}(H)$ for Nb, provided the vortex-lattice elastic moduli remain well-behaved. In this scenario, the FC disordered phase seen here (as well as in $[3,4]$ ) is a supercooled liquid and the thermodynamic ground state is an ordered solid across the entire peak-effect regime. The third scenario places $T_{\mathrm{m}}$ at or below the onset of the peak effect.

To experimentally determine the ground state and approximate value of $T_{\mathrm{m}}$, the susceptibility coil was used to shake the vortex assembly, using SANS to observe how the vortex structure evolves. The data show that above $T_{\mathrm{p}}$ the Bragg peaks start to disappear within the first $10^{2} \mathrm{sec}$ of the shaking experiment, demonstrating that the equilibrium state is disordered. Similiarly, the FC disordered states for $T<T_{\mathrm{p}}$ are metastable and the ordered ZFC state is the ground state, opposite to that for $T>T_{\mathrm{p}}$. In the $T<T_{\mathrm{p}}$ regime, though, the metastability is obviously stronger since a much larger ac field is needed to change the metastable state.

We conclude that for $T>T_{\mathrm{p}}$ the ordered $\mathrm{ZFC}$ vortex lattice is a superheated state and the ground state of the vortex system is a disordered vortex liquid, while for $T<T_{\mathrm{p}}$ the ground state is a vortex Bragg solid and the disordered FC state is a supercooled vortex liquid. A thermodynamic phase transition must therefore have taken place, with $T_{\mathrm{m}} \approx T_{\mathrm{p}}$. These results also imply the absence of superheating in conventional transport experiments with a large drive current, which solves a longstanding puzzle in which the history dependence of the nonlinear resistance always vanishes at $T_{\mathrm{p}}(H)$; only with extremely low drive currents may one then observe the subtle effects of superheating in transport.

\section{REFERENCES}

[1] For a review, see G. Blatter, Rev. Mod. Phys. 66, 1125 (1994).

[2] X.S. Ling and J.1. Budnick, in Magnetic Susceptibility of Superconductors and Other Spin Systems, R.A. Hein, et al., eds. (Plenum Press, 1991), p.377.

[3] J.W. Lynn, et al., Phys. Rev. Lett. 72, 3413 (1994).

[4] P.L. Gammel, et al. Phys. Rev. Lett. 80, 833 (1998). 


\section{SANS MEASUREMENTS OF NANOSCALE LITHOGRAPHIC FEATURES}

$\mathbf{T}$ he continued growth of the semiconductor industry depends on advances in lithographic processes and materials to enable the economical production of smaller device features. Precise measurement of the size and quality of lithographically prepared features is critical as their sizes continue to decrease, with dimensions approaching $100 \mathrm{~nm}$. Current microscopy-based techniques such as scanning electron microscopy (SEM) and atomic force microscopy (AFM) often require special modifications to enable the measurement of either the critical dimensions or feature resolution parameters. More importantly, these techniques become extremely challenging as feature sizes continue to decrease.

In this highlight, we demonstrate the powerful use of smallangle neutron scattering (SANS) to quickly, non-destructively, and quantitatively characterize both the size and profile of lithographically prepared structures as prepared on a silicon wafer substrate [1]. Until recently, SANS instruments were unable to measure lithographic feature sizes (sizes greater than $300 \mathrm{~nm}$ ) and neutron beam fluxes were insufficient to measure scattering from thin film structures. Today, with new focusing optics, the high intensity NCNR instruments allow routine SANS measurements of smaller lithographic features [2]. Other important advantages for the use of SANS to measure lithographic structures include a) the measurement of structures on silicon, because single crystal silicon wafers are generally transparent to neutrons, b) a measurement metric statistically averaged over an area of several square centimeters, and c) less stringent SANS instrument requirements as lithographic structures decrease in size.

As an example, periodic, equally spaced, parallel line patterns with a nominal size of $150 \mathrm{~nm}$ were prepared on a silicon single crystal wafer using standard $248 \mathrm{~nm}$ optical lithography, and placed directly in and normal to the neutron beam. Quantitative measurements of the size and average profile of these lines are extracted from the scattering data. SEM micrographs of these structures are shown in Fig. 1.

The SANS measurements were performed on the NG-7 30 m SANS line under ambient atmospheric conditions at the NCNR.
Newly developed neutron focusing optics consisting of 28 biconcave $\mathrm{MgF}_{2}$ lenses were used to access small enough angles to resolve feature sizes up to $300 \mathrm{~nm}$, a previously inaccessible length scale for SANS. In this configuration, the SANS data provide quantitative information about the line repeat distance and the quality of the line structure.

The 2-D scattering data from the structures displayed in Fig. 1 are shown in Fig. 2. The line structures are aligned with the vertical
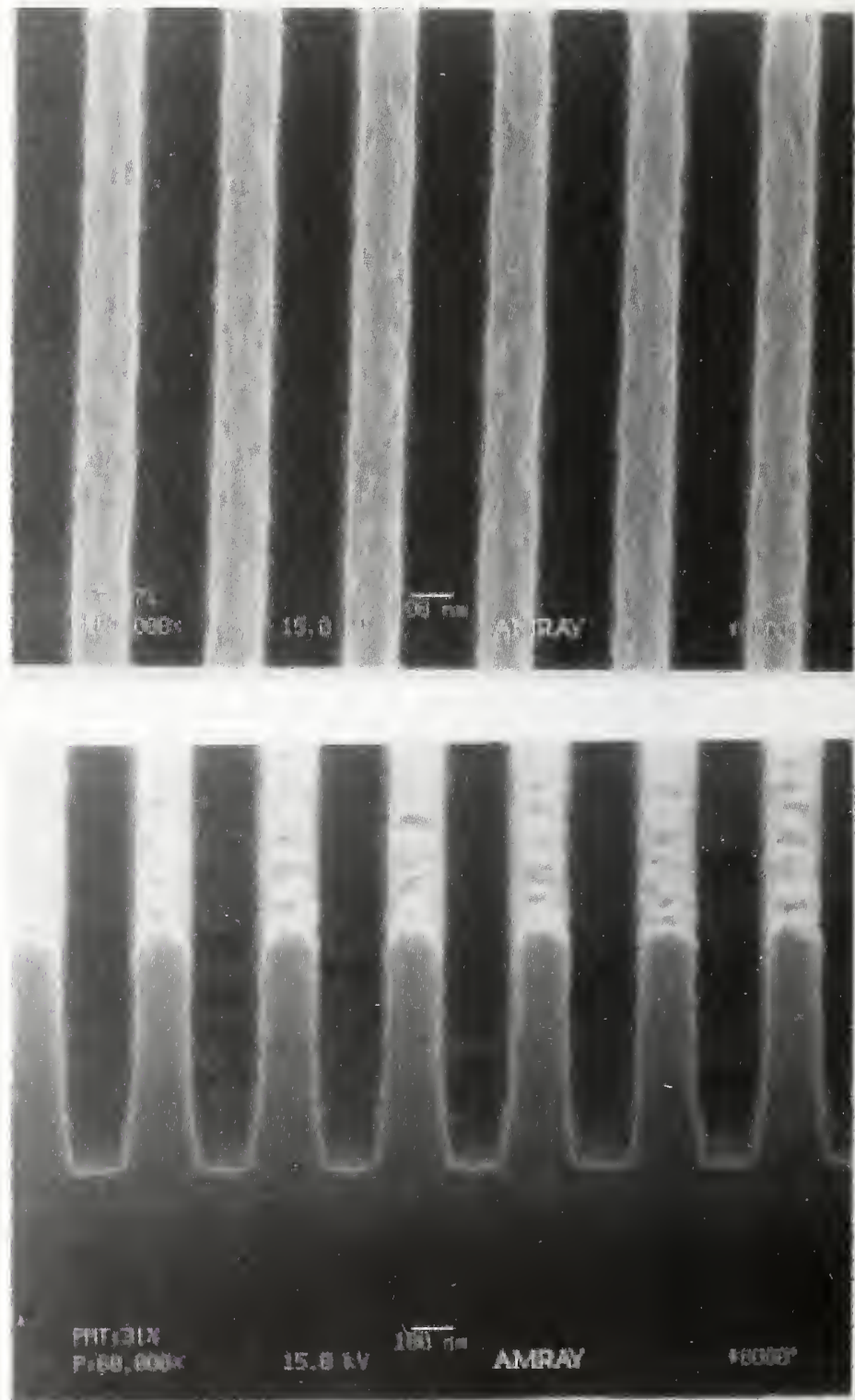

FIGURE 1. Top-down and side view scanning electron micrographs of the lithographically prepared lines used in the SANS measurement. The lines are nominally $150 \mathrm{~nm}$ wide and $0.62 \mu \mathrm{m}$ in height. 
Wen-li Wu and Eric K. Lin

Polymers Division

National Institute of Standards and Technology

Gaithersburg, MD 20899-8541
Qinghuang Lin and Marie Angelopolous

IBM

T. J. Watson Research Center

Yorktown Heights, NY 10598 axis of the detector. Six orders of diffraction peaks are immediately observed in the horizontal axis of the detector because of the highly periodic pattern of the fabricated lines. By linearly fitting the peak position plotted as a function of the diffraction order index, the feature repeat distance for the structure in Fig. 1 is determined to be $(3031 \pm 9) \AA$.

A more detailed analysis provides a quantitative determination of the average profile of the line structures, including a measure of the line-edge roughness (LER). We model the periodic line pattern as a convolution of a periodic delta function with the average cross-section of a line. In Fig. 3, the scattering intensity of a given diffraction peak is plotted as a function of the position of the peak. The solid line is the best theoretical fit to the experimental data and corresponds to a measure of the LER of $(213 \pm 13) \AA$. Also in Fig. 3 , the second and fourth order diffraction peaks are visible and less intense than the first and third diffraction peaks. The measurable intensity of the even order diffraction peaks indicates that the line feature size is slightly less than one half the overall repeat distance. The model fit results in a line feature size of $(1350 \pm 60) \AA$.

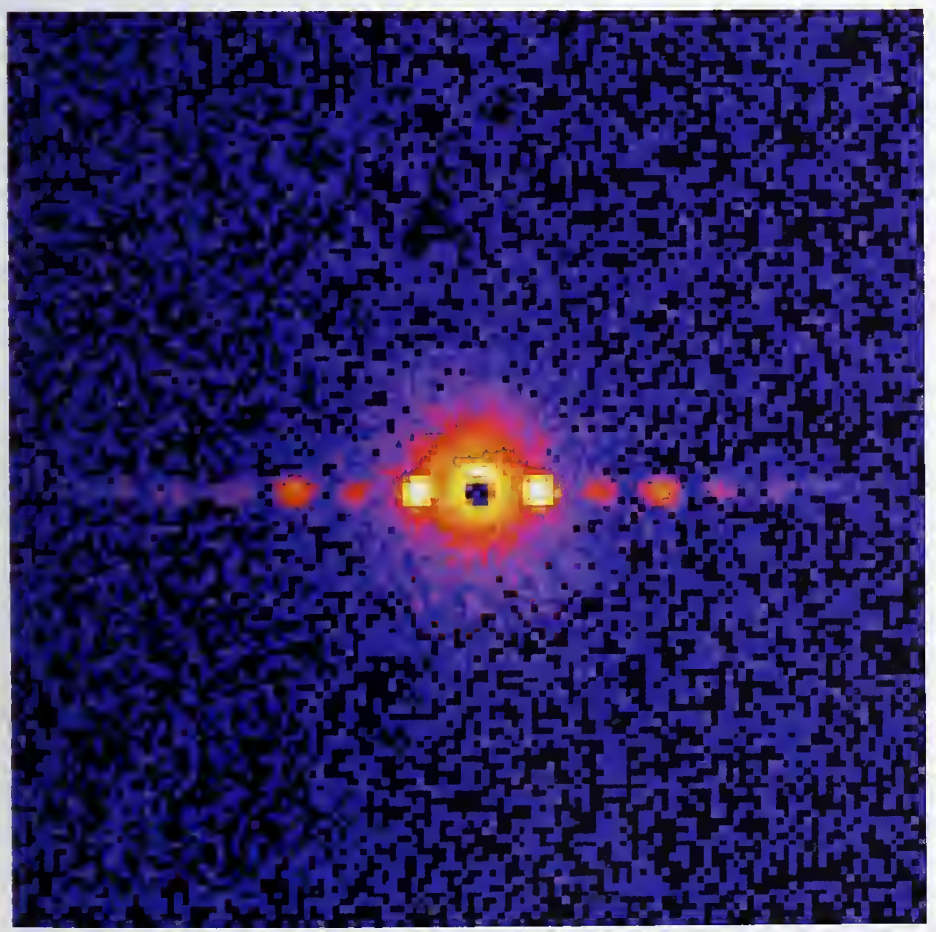

FIGURE 3. Scattering Intensity of each diffraction peak as a function of the peak position. The solid line represents the best theoretical fit to the data.

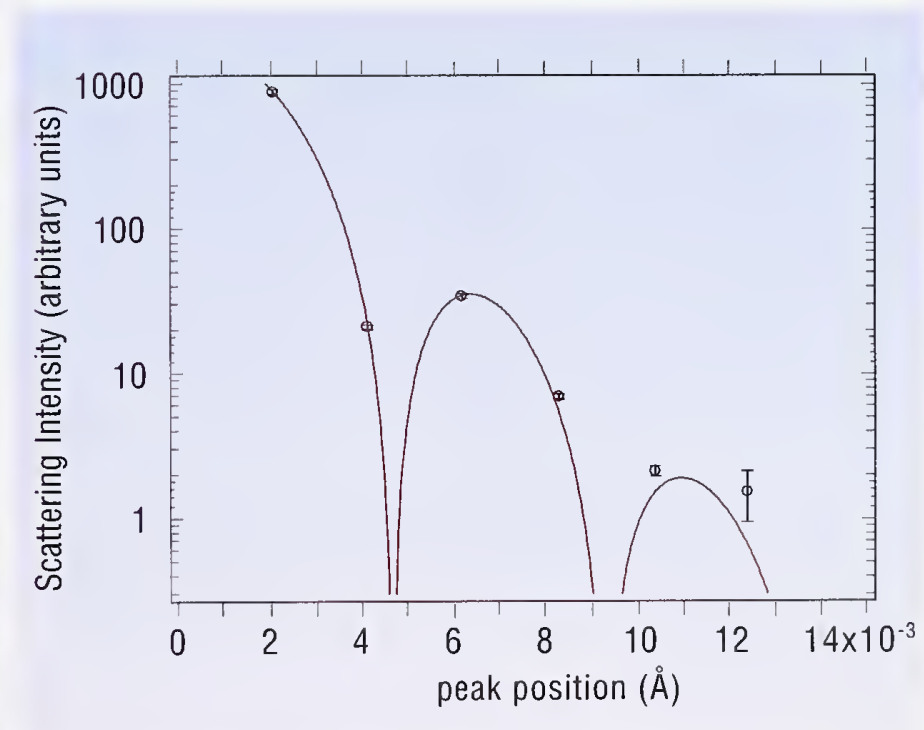

The average line structural size and cross-section were determined in a configuration where the sample was placed perpendicular to the incident neutron beam. More three-dimensional information about the average line structure can be obtained by tilting the line pattern with respect to the incident beam. Varying projections of the line profile onto the detector plane provide an elegant method to deduce more specific structural information. In additional, the formalism to extend the SANS theoretical framework to arbitrary shapes is well established and will be applied in the future. With these advances, SANS may be used to identify resolution limits in new nanofabrication processes and materials and to serve as an important metrology tool in understanding the physical processes that control the resolution of these methods.

\section{REFERENCES}

[1] W. L Wu, E. K. Lin, Q. Lin, and M. Angelopolous, J. Appl. Phys., 88, (2000) (in press).

[2] S.-M. Choi, J. G. Barker, C. J. Glinka, Y. T. Cheng, and P. L. Gammel, J. Appl. Cryst. 33, 793 (2000). For a brief description of the lens system, see the highlight in NCNR 1999 Accomplishments and Opportunities, NIST SP 944, p. 14.
FIGURE 2. Two-dimensional SANS pattern from the sample shown in Fig. 1. Six orders of diffraction are observed due to the high resolution of the lithographically prepared pattern. 


\section{ASYMMETRIC MAGNETIZATION REVERSAL IN EXCHANGE-BIASED HETEROSTRUCTURES}

xchange bias refers to a shift of the ferromagnetic hysteresis

loop along the field axis, by an amount $H_{e}$ (see Fig. $I$ for an example.) The bias is a consequence of an exchange interaction across the interface between dissimilarly ordered magnetic materials, e.g. a ferromagnet and an antiferromagnet (AF). This exchange interaction induces a unidirectional anisotropy as the AF material is cooled through its Néel temperature, $T_{\mathrm{N}}[1,2]$. Exchange bias is an example of a bulk property whose fundamental origin is attributed to physical processes occurring at the nanometer length-scale. This phenomenon is not simply a scientific curiosity; it underpins present-day magnetic recording technology.

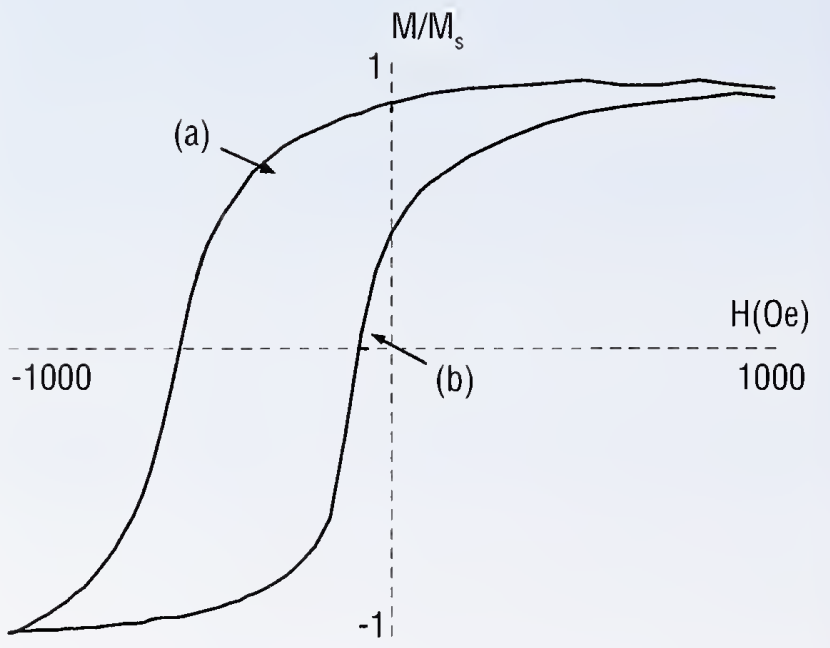

FIGURE 1. Magnetization versus applied magnetic field. Exchange bias is the shift of the ferromagnetic hysteresis loop (in this case to negative $\boldsymbol{H}$ ) from being centered at $\boldsymbol{H}=\mathbf{0}$. Measurements displayed in Figs. 2 and 3 were taken, respectively, near the coercive fields at (a) and (b). Large exchange bias is correlated with asymmetry in the magnetization reversal processes, as noted in those figure captions.
Read-write heads used with magnetically stored data are based on giant magnetoresistance (GMR) sensors. These sensors consist of layers of ferromagnetic thin films separated by nonferromagnetic ones. When the the magnetizations in the ferromagnetic layers are all oriented the same way, conduction electrons pass through them relatively easily, but when the electrons must cross from films having one orientation to another they encounter more resistance through magnetic scattering. GMR arises when an external field can change the relative orientations of the magnetization in the films easily. To keep the layers from all reorienting together in the presence of an external field, some of them must be pinned. One way to accomplish pinning is exchange biasing.

Despite its technological importance, theoretical models are unable to convincingly explain observations of exchange bias (e.g. positive exchange bias), and phenomena associated with it. Even in the simplest experimental systems such as Fe on $T M \mathrm{~F}_{2}$ where $T M=\mathrm{Mn}$ or $\mathrm{Fe}$, the asymmetric reversal of magnetization and the unusual temperature dependence of coercivity are not well understood.

Using polarized neutron reflectometry, we recently examined the magnetization reversal processes of a ferromagnetic Fe film exchange-coupled to twinned $\mathrm{AF}\left(T M \mathrm{~F}_{2}\right)$ films as a function of magnetic field [3]. Neutron scattering measurements typical of those from a sample exhibiting large exchange bias are shown in the Figs. 2 and 3 for fields at coercivity on either side of the loop. Spin-flip (SF) scattering observed on the left hand side of the loop indicates magnetization reversal via magnetization rotation. Lack of SF scattering on the right hand side is consistent with domain nucleation (with opposite magnetization) and growth. These two fundamentally different (asymmetric) reversal processes have distinct neutron scattering signatures. The ability to discern so easily between these processes sets neutron scattering apart from magnetometry.

Comparisons of measurements like those in the Figs. 2 and 3 taken from many samples, including single crystalline and polycrystalline AF films, lead to the following picture: In the case of samples with twinned AF"s, which exhibit large exchange bias, " $45^{\circ}$ exchange coupling" is energetically favorable as each AF domain independently tends to perpendicular coupling but is frustrated due to 
M.R. Fiizsimmons, A. Hoff́mann, and P.C. Yashar
C. Leighton and I. K. Schuller Department of Physics

University of California - San Diego La Jolla, CA 92093-0319
C.F. Majkrzak and J.A. Dura NIST Center for Neutron Research National Institute of Standards and Technology Gaithersburg, MD 20899-8562

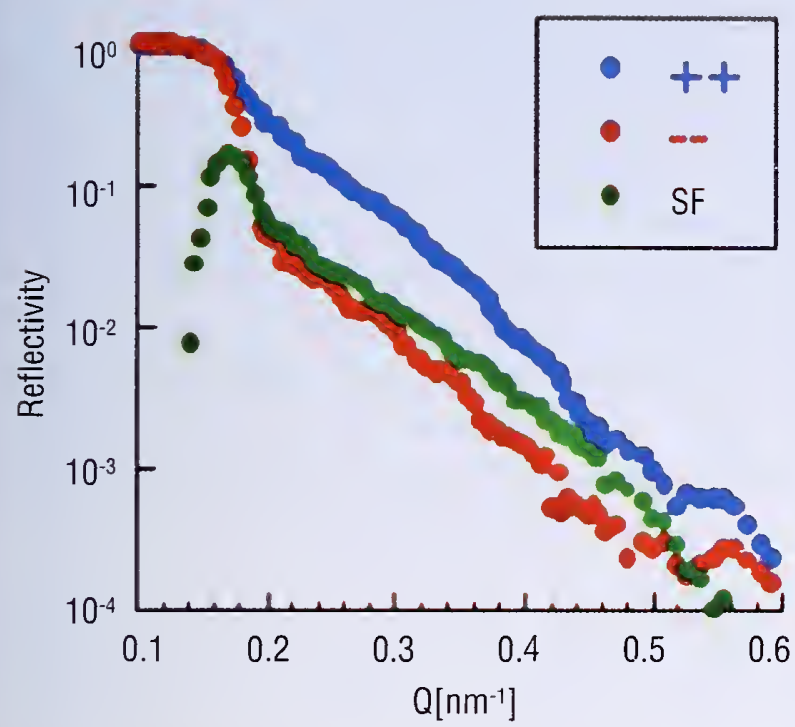

FIGURE 2. Reilectivity of scattered neutrons experiencing no flip in spin $(++$ and --$)$ and for which the spin is flipped (SF) versus $Q$. These data were taken at the coercive field on the (a) side of the loop in Fig. 1. The spin-flip scattering observed in this region indicates magnetization reversal through rotation.

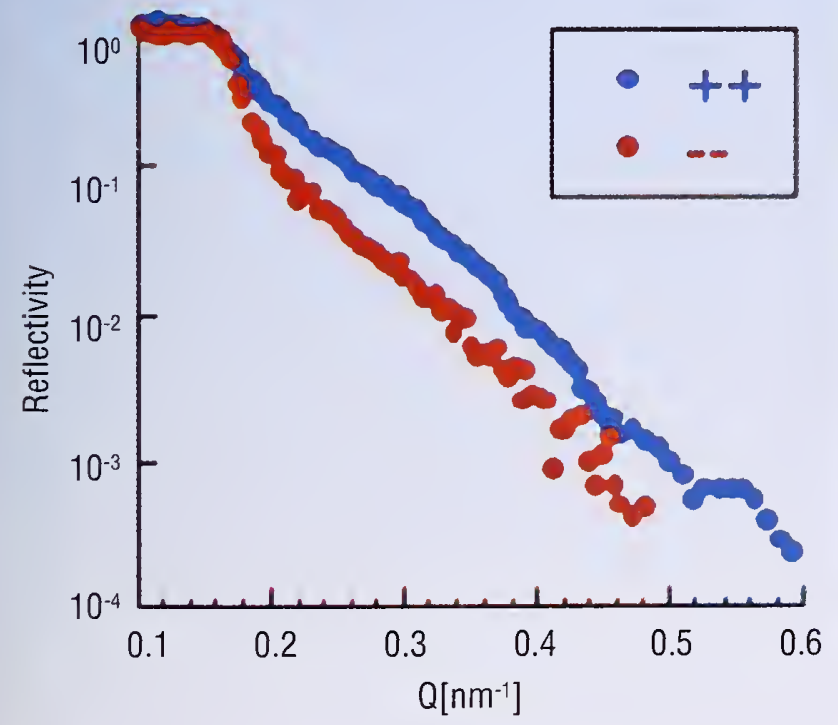

FIGURE 3. Reflectivity of scattered neutrons experiencing no flip in spin ( ++ and - -) versus $Q$. These data were taken at the coercive field on the (b) side of the loop in Fig. 1. No spin-flip scattering was observed, indicating magnetization reversal via domain nucleation and growth. the twinned microstructure. Furthermore, field cooling provides an additional unidirectional asymmetry. Therefore, field reduction from positive saturation results in magnetization rotation rather than domain nucleation. This is due to the intrinsic unidirectionality that hinders formation of domains with magnetization anti-parallel to the cooling field direction. As the field is reduced from negative saturation, formation of domains with magnetization parallel to the initial cooling direction is favored. Hence reversal occurs by domain nucleation and propagation.

For the case of samples with single crystalline (untwinned) AF's, frustration is lacking; consequently, there is no anisotropy axis parallel to the cooling field with which unidirectional anisotropy can be established. In this case, magnetization rotation is always favored (as evidenced by SF scattering on both sides of the ferromagnetic hysteresis loop). We note the exchange bias for the single crystal sample is always small. A clear correlation was observed: samples with an asymmetric magnetization reversal process exhibit large exchange bias, while those with symmetric magnetization reversal process exhibit small exchange bias.

By identifying the mechanisms involved in the asymmetry favoring large exchange biasing in this system, these and related neutron reflectivity studies point out a direction for the design of next generation GMR sensors having substantial improvements in magnetic field sensitivity.

This work was supported by the U.S. Department of Energy, BES-DMS under Contract No. W-7405-Eng-36, grant DE-FG03-87ER-45332, and funds from the University of California Collaborative University and Laboratory Assisted Research.

\section{REFERENCES}

[I] W.H. Meiklejohn and C.P. Bean, Phys. Rev. 105, 904 (1957).

[2] J. Nogués and Ivan K. Schuller, J. Magn. Magn. Mat. 192, 203 (1998).

[3] M. R. Fitzsimmons, P. Yashar, C. Leighton, Ivan K. Schuller, J. Nogues, C.F Majkrzak, and J.A. Dura, Phys. Rev. Lett. 84, 3986 (2000). 


\section{THE ROLE OF THE CRP/CAMP PROTEIN COMPLEX IN DNA TRANSCRIPTION}

D NA molecules direct the synthesis of specific RNA and protein molecules. In the early stages of protein synthesis, specific regions of the DNA (genes) are copied into short strands of RNA that retain all of the genetic information of the DNA sequence from which they were copied. The process by which RNA molecules are synthesized from the coding regions of DNA is known as DNA transcription. The RNA polymerase enzyme, whose function is to make a RNA copy of a DNA sequence, catalyzes the synthesis of these RNA molecules. The amount of RNA made from a particular region of DNA is controlled by gene regulatory proteins that bind to specific sites on DNA close to the coding sequences of a gene. In this highlight we describe experiments addressing how a particular gene regulatory protein controls RNA transcription from DNA.

One useful model of such a protein is the cyclic AMP receptor protein (CRP) of E. coli. Upon binding cyclic adenosine monophosphate (cAMP), CRP undergoes a conformational change that, in turn, promotes binding to specific DNA sequences. The CRP/ cAMP complex, upon binding DNA, produces a bend in the DNA that causes it to wrap around the RNA polymerase to promote DNA transcription.

A method well suited to directly study the structure of proteins and DNA in solution, where transcription takes place, is small-angle neutron scattering (SANS). The radius of gyration, $R_{\mathrm{g}}$, which can be used to measure conformational changes, and the structure of the molecule in solution can be determined from an analysis of the scattered neutron intensity versus $Q$.
Particularly powerful is the contrast variation technique [1] in which isotopic substitution of $\mathrm{D}$ for $\mathrm{H}$ in the solvent is routinely used to change the scattering from a macromolecule without affecting its biochemistry. In the case of a two-component complex such as CRP/cAMP/DNA (cAMP is considered to be part of the CRP component), the neutron scattering length density of CRP is quite different from that of DNA. In this case, the scattered intensity at each $Q$ value is expressed as the sum of three terms, each of which is the product of an unknown component intensity and a known contrast term. (The contrast is the difference between the scattering length density of a component and that of the solvent.) Thus, the scattering from the complex in solution can be separated into component intensities by measuring the scattered intensity of the complex, $I(Q)$, at a minimum of three contrasts obtained from different $\mathrm{D}_{2} \mathrm{O} / \mathrm{H}_{2} \mathrm{O}$ buffer mixtures.

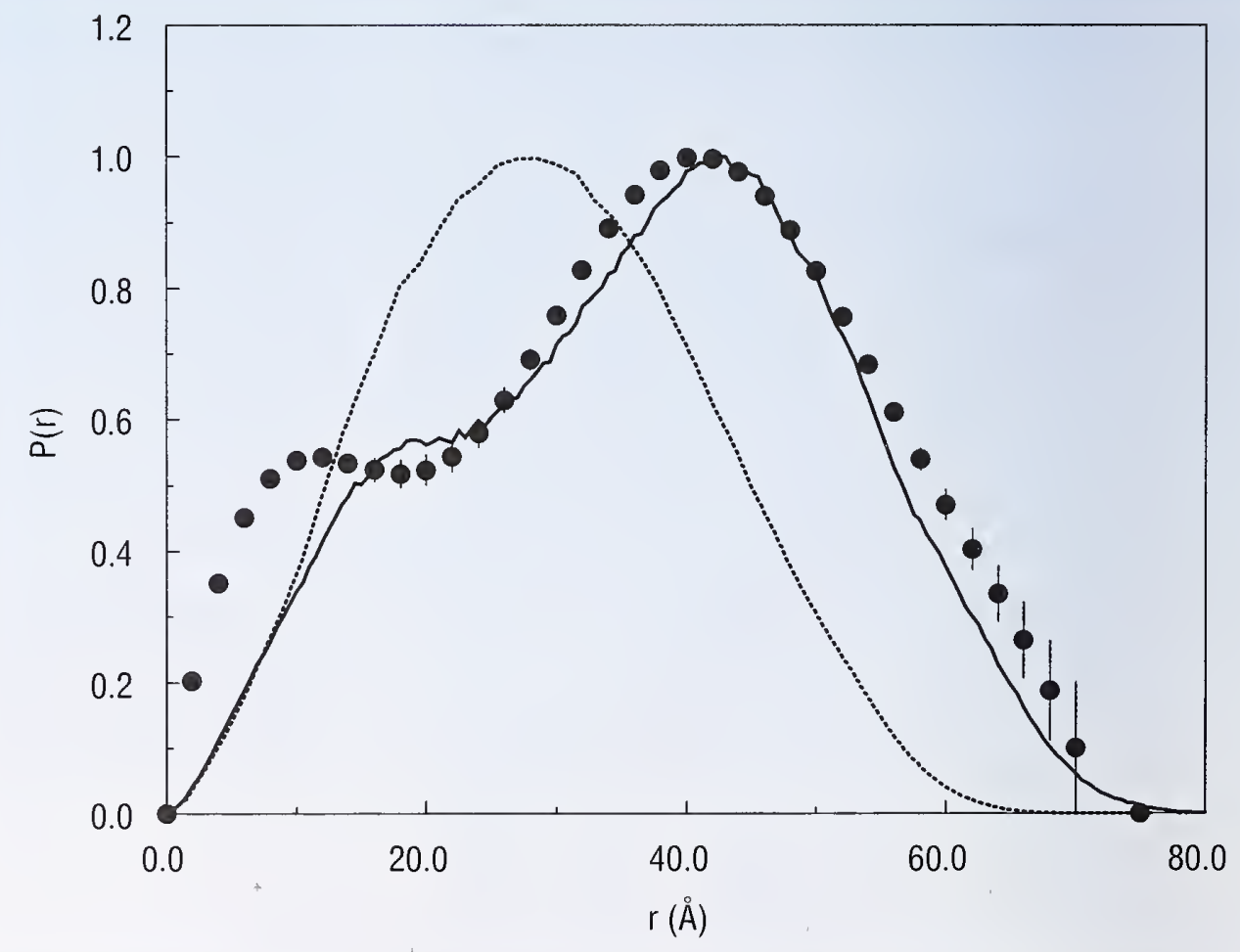

FIGURE 1. The distance distribution functions, $P(r)$, of the CRP component of the CRP/CAMP/DNA complex obtained from the SANS data $(\bullet)$, the energy-minimized $x$-ray crystal structure $(\ldots)[5,6]$ and the energyminimized model structure $(-)$. 


\section{Department of Chemistry}

University of Maryland, Baltimore County Baltimore, MD 21250
S. Wang, Y. Shi, F.P. Schwarz Center for Advanced Research in Biotechnology Rockville, MD 20850
B. Wladkowski

Department of Chemistry Western Maryland College Westminister, MD 21157
Recent SANS measurements of CRP/DNA complexes [2] confirmed, in solution, the bending of the bound DNA that was observed in an early x-ray crystal structure of the complex [3]. However, the $R \mathrm{~g}$ value for the complex was larger than that predicted from the same crystal structure. SANS confirmed experimentally that this value does not change with concentration. Thus, the increase in $R_{\mathrm{g}}$ is not due to aggregation, but it could result from an increase in the $R_{\mathrm{g}}$ of the CRP component upon DNA binding. Such a conformational change would be apparent in the SANS solution measurements: it was not evident in the crystal structure [3].

To obtain the scattered neutron intensity of the CRP component, a contrast variation series of SANS measurements was per-

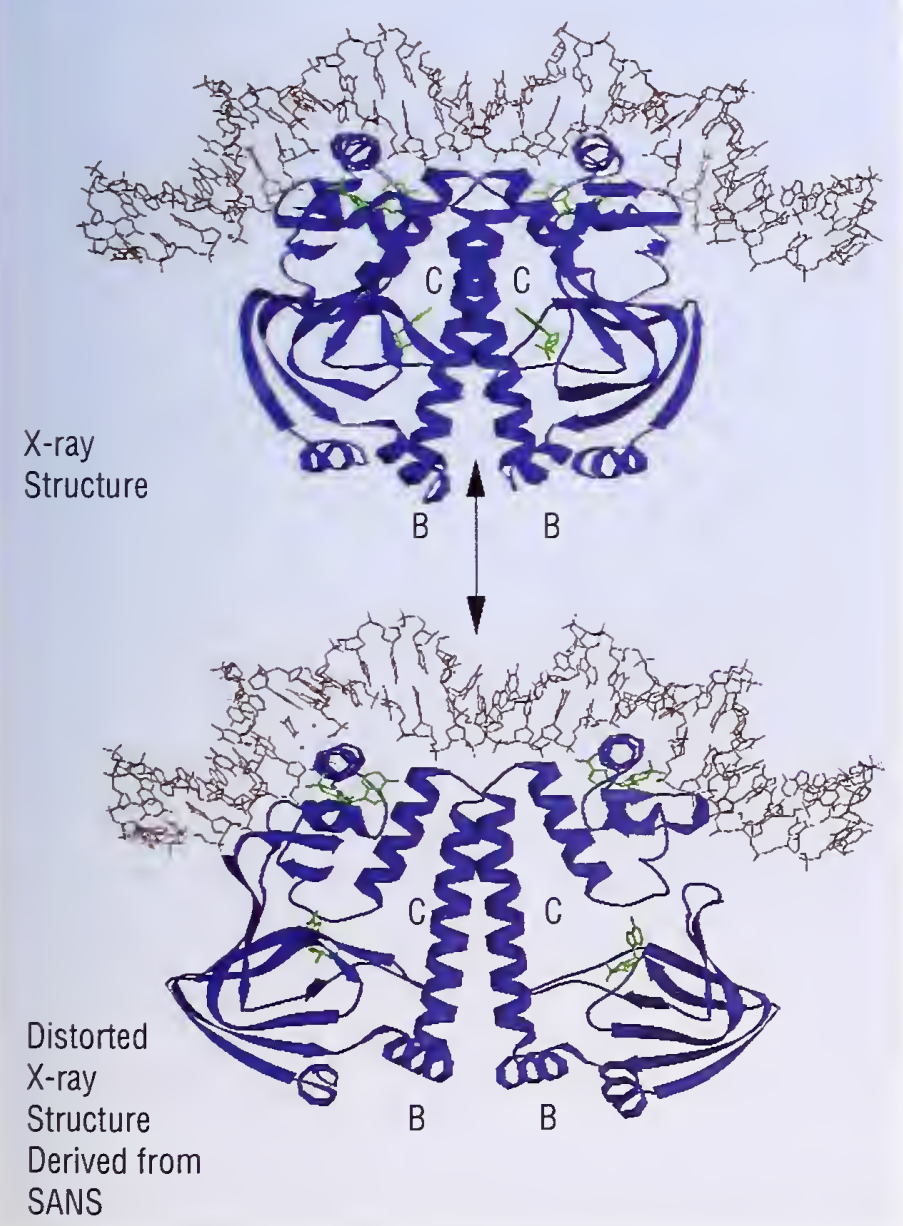

formed on CRP/DNA complexes in $0 \%, 15 \%$, and $70 \% \mathrm{D}_{2} \mathrm{O} / \mathrm{H}_{2} \mathrm{O}$ buffer solutions. The $R \mathrm{~g}$ values were found to be the same, (28 to $30 \AA$ ). for all three cases. This clearly indicates that the CRP component is the main reason that $R_{\mathrm{gCRP}}$ was larger than originally expected. It was found from the $Q$ behavior of the CRP component intensity that $R_{\mathrm{gCRP}}=28.5 \pm 0.3 \AA$, which is $\approx 6 \AA$ larger than the $21.6 \pm 0.2 \AA$ value observed in solution for CRP alone [4]. It is also $\approx 6 \AA$ larger than the $22.6 \AA$ value predicted for the CRP component from an energy-minimized $\mathrm{x}$-ray crystal structure of the complex by Parkinson et al. [5], with cAMP incorporated as in Passner and Steitz [6].

To model the solution structure of the CRP/DNA complex, the energy-minimized $\mathrm{x}$-ray structure $[5,6]$ was distorted in the regions thought most likely responsible for the conformational change in CRP upon DNA binding [7]. The distance distribution function, $P(r)$, was calculated [4] from the energy-minimized distorted conformation and compared to that obtained from the SANS data. As shown in Fig. 1, the $P(r)$ function calculated from the model structure clearly fits the experimental data better than that from the $x$-ray crystal structure $[5,6]$. A molecular representation of the energy-minimized $x$-ray crystal structure $[5,6]$ is shown in Fig. 2, along with the model structure that fits the SANS data.

The experimentally observed conformational change in CRP upon DNA binding may play a role in the enhancement of transcription of DNA by CRP. Perhaps this occurs through its contacts with RNA polymerase that is bound on the DNA at a site adjacent to the CRP binding site. This is the subject of further ongoing SANS studies.

\section{REFERENCES}

[1] H. B. Stuhrmann and A. J. Miller, Appl. Cryst. 11, 325 (1978).

[2] Y. Shi, S. Wang, S. Krueger, and F. P. Schwarz, J. Biol. Chem. 274, 6946 (1999).

[3] D. B. McKay, and T. A. Steitz, Nature 290, 744 (1981).

[4] S. Krueger, I. Gorshkova, J. Brown, J. Hoskins, K. H. McKenney, and F. P. Schwarz, J. Biol. Chem. 273, 20001 (1998).

[5] G. Parkinson, C. Wilson, A. Gunasekera, Y. W. Ebright, R. E. Ebright, and H. M. Berman, J. Mol. Biol. 260, 395 (1996).

[6] J. M. Passner and T. A. Steitz, Proc. Natl. Acad. Sci. USA 94, 2843 (1997).

[7] N. Baichoo and T. Heyduk, J. Mol. Biol. 290, 37 (1999).

FIGURE 2. The top molecule is the energy-minimized $x$-ray crystal structure $[5,6]$ of the CRP(blue)/CAMP(green)/DNA(brown) complex. The bottom molecule is the energy-minimized model structure which fits the SANS data. 
$\mathrm{n}$ recent years supercritical fluids (SCFs), materials at tempera-

tures and pressures above their critical values, are being used in both traditional industry and new advanced technical areas. The major advantage of SCFs is that their physical properties such as dielectric constant, density, and solubility parameters, can be tuned simply by adjusting the temperature and pressure. Especially, SCFs have also been shown to be effective plasticizers as well as solvents for numerous polymers. In particular, much attention has been focused on $\mathrm{CO}_{2}$ since it becomes supercritical at a moderate critical temperature and pressure, $T_{\mathrm{c}}=31.3{ }^{\circ} \mathrm{C}$ at $P_{\mathrm{c}}=73.8 \mathrm{bar}$, and it is environmentally benign [1].

In spite of its practical importance and numerous studies of the $\mathrm{CO}_{2}$-induced swelling in bulk polymers, fundamental questions still remain. It is important to understand the interaction of supercritical $\mathrm{CO}_{2}\left(\mathrm{scCO}_{2}\right)$ that can modify diffusion coefficient and the glass transition in thin polymer films. The performance of the many applications of thin films is often dependent on knowledge of the structure and dynamics of the interfaces. Therefore, research in this area will advance understanding of the fundamental physics and applications of polymer thin films.

Neutron reflectivity (NR) is used for quantitative determination of the thicknesses, compositions, and interfacial structures of polymer thin films on a nanometer scale. To achieve this under in situ conditions, we have developed a temperature and pressure controlled chamber specifically for neutron reflectivity (Fig. 1). The cell is equipped with two cylindrical sapphire windows. $\mathrm{CO}_{2}$ is loaded into the cell by means of a manually operated pressure generator. Pressurizing and depressurizing cycles up to 1400 bar are possible. Temperature and pressure stability of the chamber of $\pm 0.1{ }^{\circ} \mathrm{C}$ and $\pm 0.2 \%$, respectively can be achieved. Due to the high absorption of neutrons in $\mathrm{CO}_{2}$, the incident and reflected beams passed through the Si substrate with a transmission of

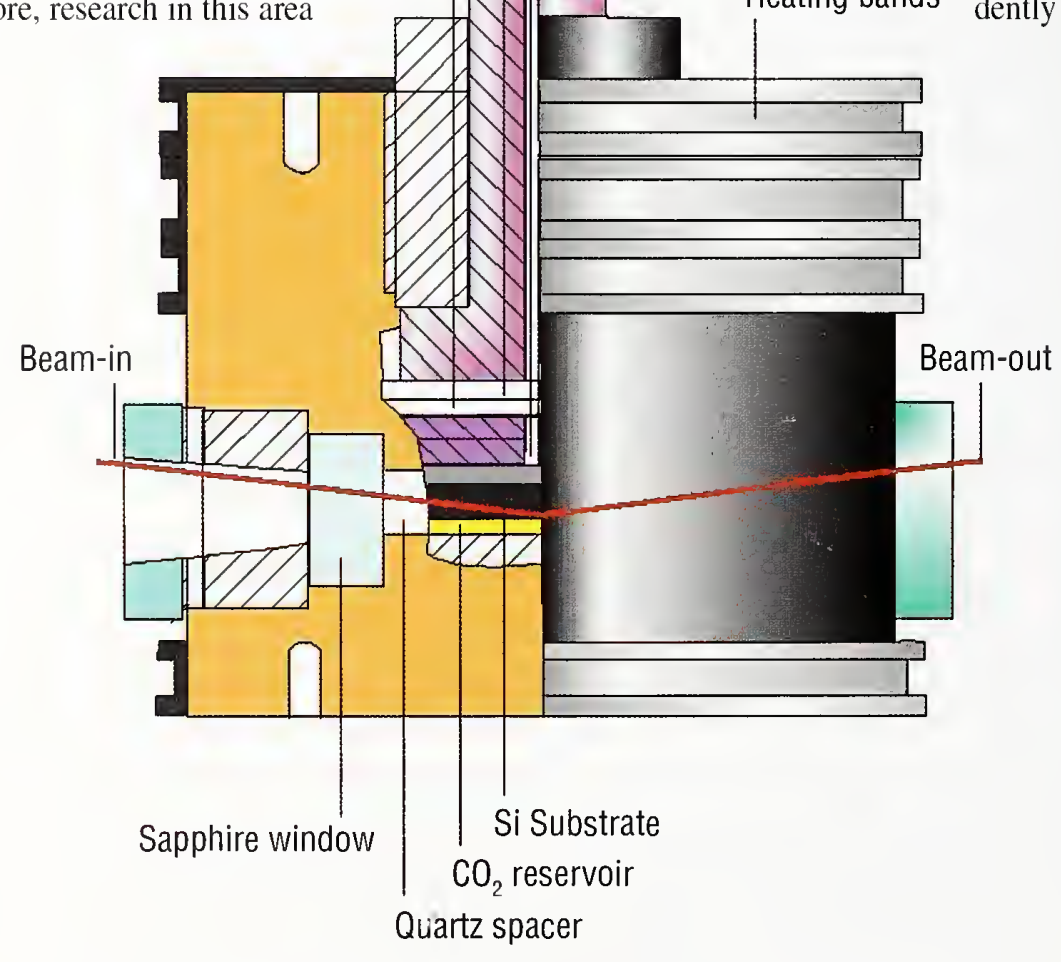

FIGURE 1. Cross-sectional view of the supercritical $\mathrm{CO}_{2}$ chamber for neutron reflectivity measurements. 
K.Shin, T.Koga, Y.Seo, Y.Zhang, M.Rafailovich, and J.Sokolov

Department of Materials Science and Engineering State University of New York at Stony Brook

Stony Brook NY, 11794-2275
S.K.Satija

NIST Center for Neutron Research

National Institute of Standards and Technology

Gaithersburg, MD 20899-8562
In this study we have successfully performed NR measurements of deuterated polystyrene (d-PS) thin films in $\mathrm{CO}_{2}$ at $P<$ 700 bar (Fig. 2). The results show an anomalously large swelling maximum $(\Delta V / N 25 \%)$ which occurs only at the supercritical fluid/gas phase boundary. Atomic force microscopy confirmed the swelling is uniform throughout the films and does not produce large voids. Such a swelling maximum at the supercritical fluid/gas phase boundary has never been seen in bulk PS- $\mathrm{CO}_{2}$ mixtures. In addition, an isobaric experiment at 79 bar showed that the same swelling behavior did not appear at the phase boundary between the liquid and supercritical fluid $\mathrm{CO}_{2}$ phases.

At this point we can only speculate that the anomalous swelling behavior may be related to the higher order nature of the gas/supercritical fluid transition that is accompanied by larger density fluctuations than the first-order liquid/supercritical fluid transition. Experimentally these fluctuations are clearly seen by the sharp increase in background scattering from the $\mathrm{CO}_{2}$ at the transition boundary. Adsorption of $\mathrm{CO}_{2}$ into the viscous polymer film may suppress these fluctuations thereby lowering the energy of the sys-

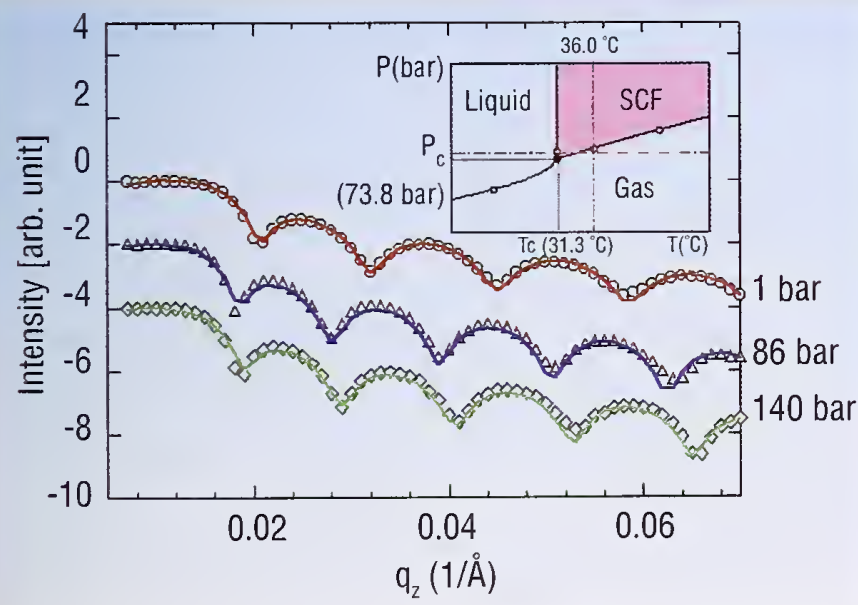

FIGURE 2. Representative reflectivity data for the first pressurization process at $T=36^{\circ} \mathrm{C}$. The lines are the best-fit results of the model to the observed density profiles. Swelling is evident in the change of the period of the fringes. Inset: a schematic phase diagram of $\mathrm{CO}_{2}$ near critical point. The vertical line at $36^{\circ} \mathrm{C}$ in the inset is the isotherm along which data were taken at pressures of 1,86 , and 140 bar. tem. This effect was further investigated by observing the swelling of the d-PS film as a function of temperature at $P=79$ bar (Fig. 3.)

Recently we have expanded our research by measuring chain mobility in bilayer polymer films of d-PS and hydrogenated PS (h-PS). We have used secondary ion mass spectrometry and NR to measure the diffusion coefficient as a function of pressure, temperature and molecular weight. This work established that swelling at the phase boundary is accompanied by an increase in interdiffusion between the polymer layers and a large decrease in the PS glass transition temperature. These results show that $\mathrm{scCO}_{2}$ can potentially be used to process multi-component thin polymer films that may otherwise not be miscible. Potential applications are in the areas of graded index of refraction waveguides and lower dielectric constant films for use in microelectronic devices.

\section{REFERENCES}

[1] M.A. McHugh and V.J. Krukonis, Supercritical Fluid Extraction, 2nd Ed., Butterworth-Heinemann, 1993.

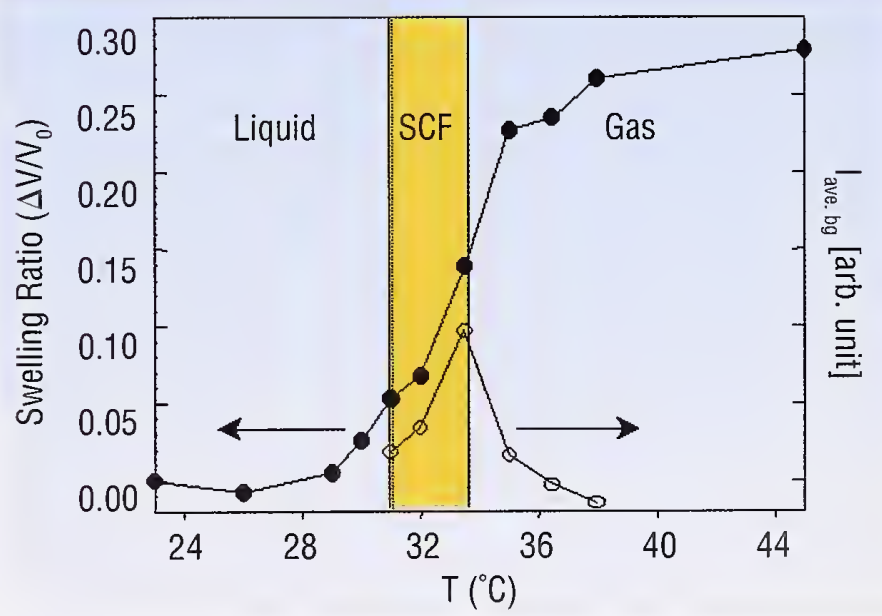

FIGURE 3. Temperature dependence of the swelling of polystyrene at $\boldsymbol{P}=\mathbf{7 9}$ bar (filled circles). Open circles show the averaged background intensity as a function of temperature. 


\section{COLD NEUTRON DEPTH PROFILING OF LITHIUM BATTERY MATERIALS}

A s part of a continuing collaboration between NIST and Tufts

University, we have employed cold neutron depth profiling (NDP) to measure elemental concentrations versus depth for several lithium ion battery materials. One goal is to rationally design the thermo-mechanical properties of amorphous lithium phosphorous oxynitride (lipon, a solid ionic conductor), particularly the thermal stress (thermal expansion coefficient). A part of this study is to relate the resulting thermal stress to starting composition and temperature of evaporant, and of the composition and pressure of the background gas during deposition. We are also studying $\mathrm{LiCoO}_{2}$ films (a material that can be used as an electrode in batteries) to determine if the ion beam assisted deposition process used has the capability of controlling not only the degree of crystallinity and orientation of crystallites, but also of the Li/Co ratio.

The NIST cold neutron depth profiling instrument and technique have been described previously [1]. The technique of neutron depth profiling (NDP) permits the determination of depth profiles in thin films up to a few micrometers for several light elements. The most readily analyzed elements are lithium, nitrogen, and boron. We have previously reported measurement of lithium mobility in electrochromic devices [2]. The lithium depth profiles are based on the measurement of the energy of alpha particles and/or tritons from the ${ }^{6} \mathrm{Li}(\mathrm{n}, \alpha)^{3} \mathrm{H}$ reaction. Nitrogen depth profiles are based on the measurement of the energy of protons from the ${ }^{14} \mathrm{~N}(\mathrm{n}, \mathrm{p})^{14} \mathrm{C}$ reaction. Samples are placed in a beam of cold neutrons, and the emerging particles are intercepted by surface barrier detectors that measure their number and energy. Comparing the emission intensity with that of a known standard leads to quantitative determination of the lithium and nitrogen concentrations. Moreover, the emitted charged particles lose energy as they exit the film; this energy loss provides a direct measurement of the depth of the originating lithium nucleus. A great advantage of the NDP technique is that it is non-destructive, which allows repeated observations of the concentrations under different conditions. When combined with other techniques, e.g., activation analysis, ratios to other constituents can be determined.

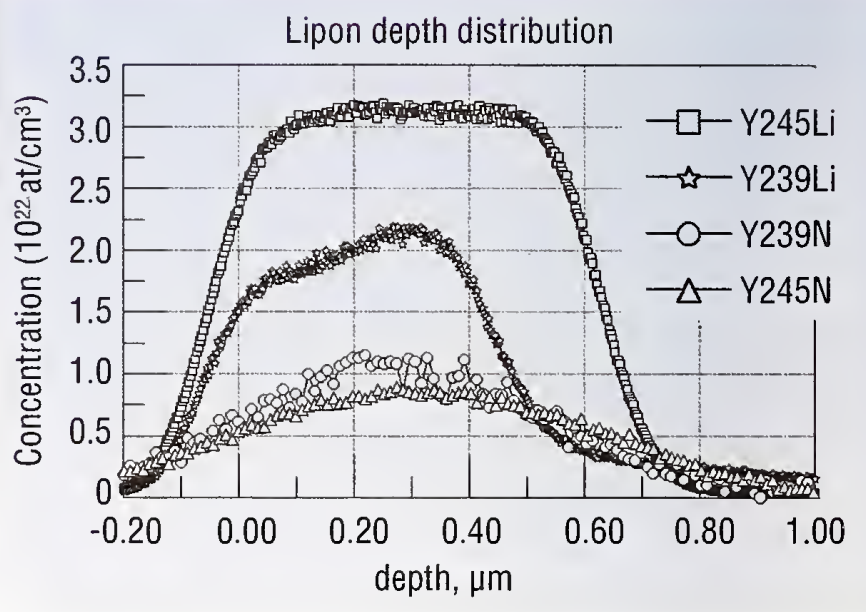

FIGURE 1. Depth profiles of lithium and nitrogen for two different lipon samples.

Table I

$\begin{array}{lcc}\text { Sample } & \text { Li/N Ratio } & \text { Thickness, } \mu \mathrm{m} \\ \text { Y243 } & 4.46 & 0.7 \\ \text { Y232 } & 4.39 & 1.5 \\ \text { Y239 } & 1.63 & 0.4 \\ \text { Y245 } & 3.1 & 0.6\end{array}$

Figure 1 gives an example of profiling results using the NDP technique. Shown here are profiles for two lipon samples manufactured under different conditions. The elemental concentrations in atoms $/ \mathrm{cm}^{3}$ are presented as a function of depth. Because the alpha particle loses energy at a greater rate than the proton, the resolution for the lithium profile is better than that for nitrogen. One observes that the sample Y245 has a much more uniform distribution of lithium than sample Y239. By integrating the areas under the curves, one obtains the total concentration of lithium and nitrogen, respectively, and therefore the ratio of the two in the sample. Table I gives measured lithium/nitrogen ratios thus obtained and the corresponding thicknesses of four lipon samples. 
G.P. Lamaze, H.H. Chen-Mayer, and
F. Vereda, A. Gerouki, N. Clay, P. Zerigian,

T. Haas, and R.B. Goldner

Electro-Optics Technology Center

Tufts University

Medford, MA 02155

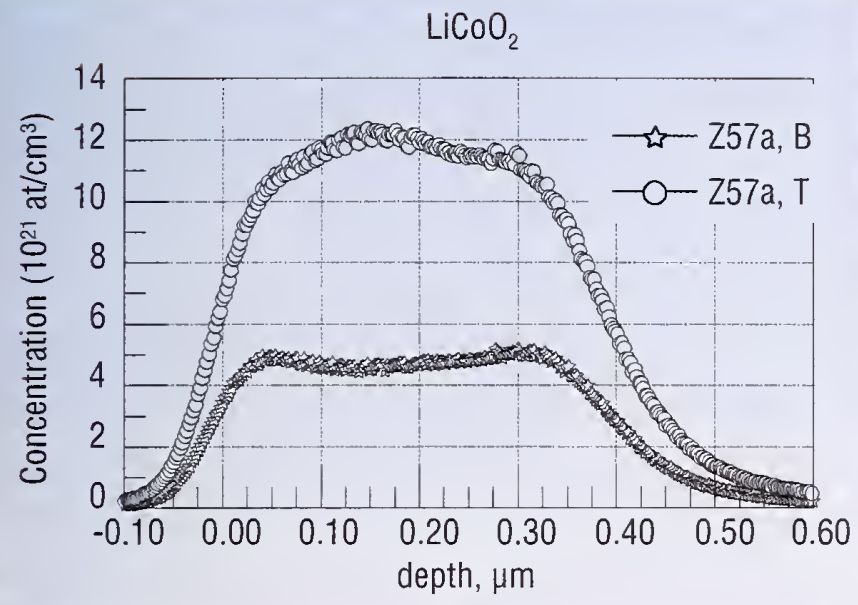

FIGURE 2. Typical lithium depth profiles of two $\mathrm{LiCoO}_{2}$ samples.

Table II

$\begin{array}{lllcl}\text { Sample } & \begin{array}{l}\text { Lithium } \\ \left(\mathrm{at}_{\mathrm{cm}}\right)\end{array} & \begin{array}{l}\text { Cobalt } \\ \left(\mathrm{at} / \mathrm{cm}^{2}\right)\end{array} & \begin{array}{l}\text { Li/Co } \\ \text { Ratio }\end{array} & \begin{array}{l}\text { Ratio } \\ \text { Uncertainty } \\ (\%)(1 \sigma)\end{array} \\ \text { Z57a,T } & 4.9 \times 10^{17} & 7.0 \times 10^{17} & .70 & 3.5 \\ \text { Z57a,B } & 2.2 \times 10^{17} & 7.0 \times 10^{17} & .31 & 3.5 \\ \text { Z57b,T } & 4.8 \times 10^{17} & 7.0 \times 10^{17} & .68 & 3.5 \\ \text { Z57b,B } & 1.9 \times 10^{17} & 7.0 \times 10^{17} & .27 & 3.5 \\ \text { Z59 } & 9.4 \times 10^{17} & 7.6 \times 10^{17} & 1.25 & 3.5\end{array}$

To obtain information on other isotopes that are not measurable by NDP, a combination of techniques is employed. In the following example the ratio of lithium to cobalt in two thin film $\mathrm{LiCoO}_{2}$ samples is determined. The lithium concentration is determined by NDP, as described above; and the cobalt concentration is determined by instrumental neutron activation analysis (INAA). Figure 2 gives lithium depth distributions from NDP measurement for two thin films of $\mathrm{LiCoO}_{2}$. The integral under the curves gives the total amount of lithium in the film. After the depth distributions were obtained, the samples were encapsulated in polyethylene "rabbits" for irradiation in the core of the NIST reactor. The total cobalt concentration was then determined by INAA in which the ${ }^{60} \mathrm{Co}$ gamma decay intensity was measured and compared with a standard. Table II gives the lithium and cobalt atom area density obtained from NDP and INAA respectively, as well as the lithium/ cobalt atom ratios for these and other samples. The INAA technique does not provide any depth information, so that the ratio values listed in the table are for the average over the entire depth.

To summarize, depth profiles of two different lithium ion battery materials have been measured. For the lipon sample, profiles were obtained for lithium and nitrogen as well as the total quantity of each of these elements in the film. To date, an insufficient number of samples have been measured to obtain a good correlation with the physical properties of the films. We are also investigating the possibilities of measuring Li/P ratios of both starting materials and resulting films by combining NDP with RNAA for phosphorous.

For the lithium cobalt oxide sample, the NDP technique was combined with INAA to determine the ratio of lithium to cobalt in the samples. Although further work is needed to better quantify the relative evaporation rates of lithium and cobalt, it has been demonstrated that the measured $\mathrm{Li} / \mathrm{C}$ ratio varies in direct proportion to the relative evaporation rates of lithium and cobalt, as anticipated. Furthermore, the results indicate that the Li/Co ratio can be controllably varied from being less than one to greater than one.

\section{REFERENCES}

[1] R. G. Downing, G. P. Lamaze, J. K. Langland, and S. T. Hwang, NIST Journal of Research. 98, 109 (1993).

[2] G. P. Lamaze, H. H. Chen-Mayer, A. Gerouki, and R. B. Goldner, Surf. Interface Anal. 29, 637 (2000). 


\section{SERVING THE SCIENTIFIC AND TECHNOLOGICAL COMMUNITIES}

$\mathrm{T}$ he role of the NCNR as a national user facility has expanded significantly over the past year, as the final few instruments envisioned in the original cold neutron project have become operational. The Disk-Chopper Spectrometer, the Filter-Analyzer Neutron Spectrometer, the High Flux Backscattering Spectrometer, and the Neutron Spin-Echo spectrometer now permit U.S. scientists to carry out neutron spectroscopy with greatly enhanced resolution and sensitivity. In addition the new thermal perfect-crystal diffractometer small angle neutron scattering instrument (USANS) has been commissioned this year, expanding the length scale available by this technique to $10^{4} \mathrm{~nm}$. User experiments show a steadily increasing diversification in subject area and technique, enabled by the new instruments. We anticipate that the trend will continue over the next few proposal cycles. (See the highlights on USANS, FANS, and DCS in this issue. The 1999 NCNR report featured a highlight on the NSE.)

User participation over the past 14 years shows continuing growth (see Fig. 1). The NCNR currently accommodates more than half of all neutron users in the United States. It has assumed greater importance to the neutron user community this year with

1800

In FY 2000 Research Participants were from:

1600 21 NIST Divisions and Offices 34 U.S. Government Laboratories

1400 1200 115 U.S. Universities 50 U.S. Industrial Laboratories

1000 800 600 400 200 0

$\begin{array}{lllllll}88 & 90 & 92 & 94 & 96 & 98 & 2000\end{array}$

FIGURE 1: Research Participants at the NCNR. the announcement of the permanent shutdown of the High Flux Beam Reactor at Brookhaven National Laboratory. As the Spallation Neutron Source is being built at Oak Ridge, the NCNR continues to be the Nation's premier facility for providing neutrons to the U.S. research community.

\section{THE NCNR USER PROGRAM}

Researchers may obtain use of NCNR neutron beam instruments in several ways, the most direct being through the formal proposal system. Approximately twice a year, a Call for Proposals is issued. After a thorough review process by external referees and by the NCNR Program Advisory Committee (PAC), approved proposals are allocated beam time. The PAC is a panel of distinguished scientists with expertise across a broad range of neutron methods and scientific disciplines. It is the body primarily responsible for proposal review and recommending user policies for the NCNR, working closely with the Center's Director and staff. Its current membership includes Sanat Kumar (Penn State University, chair), Robert M. Briber (University of Maryland), Michael K. Crawford (DuPont), Dieter K. Schneider (Brookhaven National Laboratory), Thomas P. Russell (University of Massachusetts), Sunil K. Sinha (Argonne National Laboratory), Laurence Passell (Brookhaven National Laboratory), and Gabrielle G. Long (NIST).

At the recent PAC meeting in May 2000, the PAC considered 71 proposals for SANS and reflectometry, in addition to 43 for inelastic neutron scattering. Although we expect that both categories will see increased user demand in future proposal rounds, the latter area is likely to see more growth, since the new inelastic scattering spectrometers will offer capabilities that in aggregate have not been available previously at U.S. neutron facilities.

\section{THE CENTER FOR HIGH RESOLUTION NEUTRON SCATTERING}

Several NCNR instruments are supported by the National Science Foundation (NSF) through the Center for High Resolution Neutron Scattering (CHRNS), a very important component of the user program. The instruments include a $30 \mathrm{~m}$ SANS machine, the SPINS triple-axis spectrometer, and USANS. Approximately $40 \%$ of the instrument time allocated by the PAC goes to experiments carried out on CHRNS instruments. In the near future, another SANS diffractometer, the $8 \mathrm{~m}$ machine on neutron guide $\mathrm{NG}-1$, which is presently used primarily for NIST programmatic research, will be 
upgraded to a more powerful $9 \mathrm{~m}$ instrument with a new detector, and made available to users. The NSF is currently reviewing a proposal to further expand the scope of CHRNS, so that it will encompass several of the newer cold neutron spectrometers. Including these instruments in the CHRNS project will provide maximal technical support and accessibility to the user community.

\section{SIXTH ANNUAL SUMMER SCHOOL}

The NCNR held its annual Summer School on Neutron Scattering on June 5-9, 2000. The course this year focused on the complementary techniques of SANS and neutron reflectometry (NR) and was attended by a group of 32 graduate students and postdoctoral fellows, predominantly from university chemical engineering and materials science departments. By devoting an entire week to just two techniques, it was possible to cover both theoretical and practical aspects, as well as applications, in some depth. Sixteen NCNR staff members led the participants through lectures, demonstrations and hands-on experiments at the NCNR's two $30 \mathrm{~m}$ SANS instruments and two reflectometers. Included for the first time in this year's course were demonstrations of newly developed computational tools for planning and simulating SANS and NR experiments now accessible through the NCNR Web site.

The final day of the course consisted of parallel lecture sessions in the morning on applications of the two techniques drawn from recent research in polymer science, complex fluids, magnetism, and structural biology. The course closed with a session in which representatives from each team presented their experimental results to the whole class and staff, which prompted several lively discussions. Comments received throughout the week and on the course evaluation forms indicated that the course was successful in enabling the attendees to assess the applicability of neutron scattering to their own research interests. As in the past, this summer school was jointly sponsored with the National Science Foundation, which provided financial assistance to many of the university participants.

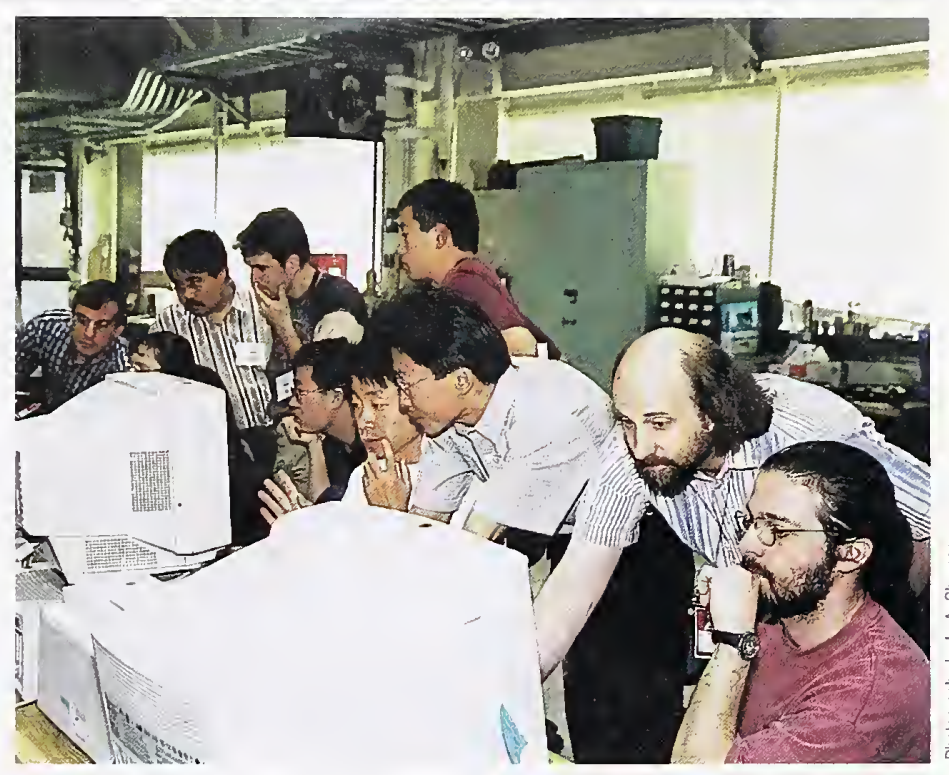

FIGURE 2: Participants gain hands-on experience in SANS measurements at the 6th annual summer school on neutron scattering, June 5-9, 2000

\section{COLLABORATIONS}

Direct collaborations on specific experiments remain a common way for users to pursue their ideas using NCNR facilities, accounting for approximately half of the number of instrument-days. The thermalneutron triple-axis spectrometers are mainly scheduled in this way. Most of the time reserved for NIST on these and all other NCNR instruments is devoted to experiments that are collaborations with non-NIST users. Collaborative research involving external users and NIST scientists often produces results that could be not obtained otherwise.

Another mode of access to the NCNR is through Participating Research Teams (PRTs). In this case, groups of researchers from various institutions join forces to build and operate an instrument. Typically, $50 \%$ to $75 \%$ of the time on the instrument is then reserved for the PRT, and the remaining time is allocated to general user proposals. For example, a PRT involving ExxonMobil, the University of Minnesota, and NIST cooperates on the NG-7 $30 \mathrm{~m}$ SANS instrument. Similar arrangements involving other PRTs apply for the horizontal-sample reflectometer, the high-resolution powder diffractometer, the filter-analyzer spectrometer, and the neutron spinecho spectrometer. 


\section{INDEPENDENT PROGRAMS}

There are a number of programs of long standing located at the NCNR that involve other parts of NIST, universities, industrial laboratories, or other government agencies.

The Polymers Division of the Materials Science and Engineering Laboratory has two major program elements at the NCNR. In the first, the purpose is to help the U.S. microelectronics industry in addressing their most pressing materials measurement and standards issues. In today's ICs and packages the feature size on a chip is ever shrinking, approaching $250 \mathrm{~nm}$, while the size of a polymer molecule is typically $5 \mathrm{~nm}$ to $10 \mathrm{~nm}$. As feature size shrinks, the structure and properties of interfaces play an increasingly important role in controlling the properties of the polymer layers used in interconnects and packages. NIST scientists use both neutron reflectivity and other neutron scattering methods to characterize polymer/metal interfaces with regard to local chain mobility, moisture absorption, glass transition temperature, and crystalline structure.

In the second program element, the objective is to understand underlying principles of phase behavior and phase separation kinetics of polymer blends, both in the bulk and on surfaces, in order to help control morphology and structure during processing. SANS and reflectivity measurements in equilibrium, in transient conditions, and under external fields, provide essential information for general understanding as well as for specific application of polymer blend/ alloy systems. Customers include material producers and users, ranging from chemical, rubber, tire, and automotive companies, to small molding and compounding companies. The focus of research on polymeric materials includes commodity, engineering and specialty plastic resins, elastomers, coatings, adhesives, films, foams, and fibers.

The ExxonMobil Research and Engineering Company is a member of the Participating Research Team (PRT) that operates, maintains, and conducts research at the NG-7 30 m SANS instrument and the recently commissioned NG-5 Neutron Spin Echo Spectrometer. The mission is to use those instruments, as well as other neutron scattering techniques, in activities that complement research at ExxonMobil's main laboratories as well as at its affiliates' laboratories around the world. The aim of these activities is to deepen understanding of the nature of ExxonMobil's products and processes, so as to improve customer service and to improve

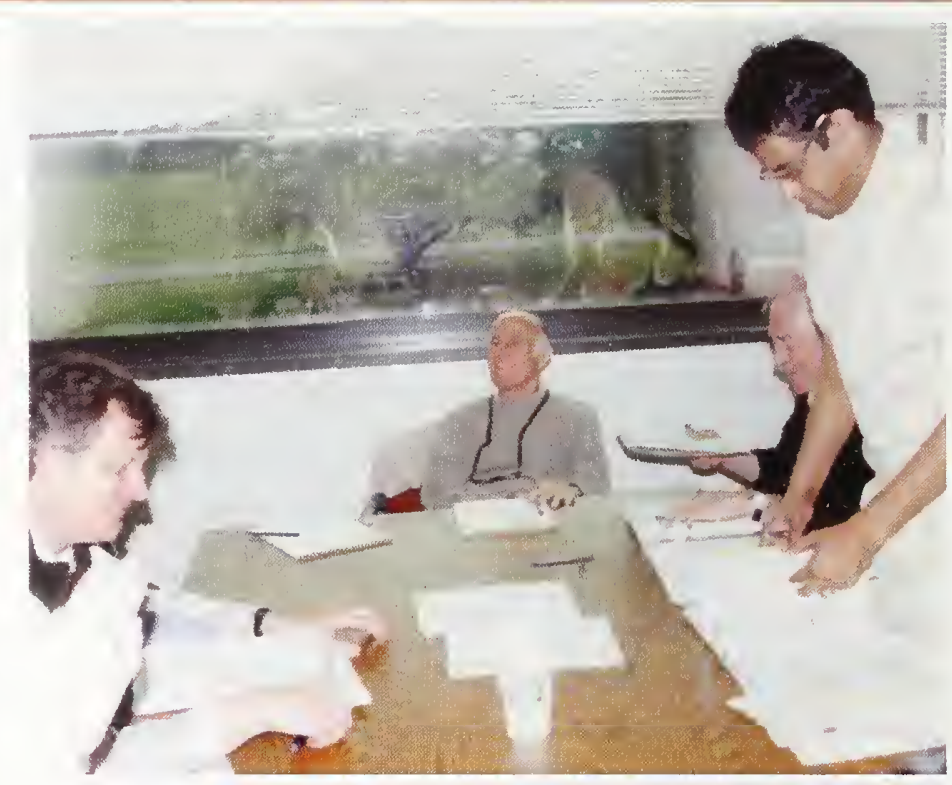

FIGURE 3: PAC substitute Bill Hamilton (ORNL), members Larry Passell (BNL) and Dieter Schneider (BNL), and PAC Chair, Sanat Kumar (Penn State U.) share a lighter moment while considering proposals for beam time at the NCNR.

the return on shareholders' investment. Accordingly, and taking full advantage of the unique properties of neutrons, most of the experiments use SANS or other neutron techniques to study the structure and dynamics of hydrocarbon materials, especially in the fields of polymers, complex fluids, and petroleum mixtures. ExxonMobil regards its participation in the NCNR and collaborations with NIST and other PRT members not only as an excellent investment for the company, but also as a good way to contribute to the scientific health of the Nation.

\section{The Nuclear Methods Group (Analytical Chemistry} Division, Chemical Science and Technology Laboratory) has as its principal goals the development and application of nuclear analytical techniques for the determination of elemental compositions with greater accuracy, higher sensitivity and better selectivity. A high level of competence has been developed in both instrumental and radiochemical neutron activation analysis (INAA and RNAA). In addition, the group has pioneered the use of cold neutron beams as analytical probes with both prompt gamma activation analysis (PGAA) and neutron depth profiling (NDP). PGAA measures the total amount of a particular analyte present throughout a sample by the analysis of the prompt gamma-rays emitted during neutron capture. NDP, on the other hand, determines concentrations of several important elements (isotopes) as a function of depth within the 
first few micrometers of a surface by energy analysis of the prompt charged-particles emitted during neutron bombardment. These techniques (INAA, RNAA, PGAA, and NDP) provide a powerful combination of complementary tools to address a wide variety of analytical problems of great importance in science and technology, and are used to help certify a large number of NIST Standard Reference Materials.

During the past several years, a large part of the Group's efforts has been directed towards the exploitation of the analytical applications of the guided cold-neutron beams available at the NIST Center for Neutron Research. The Group's involvement has been to design and construct state-of-the-art cold neutron instruments for both PGAA and NDP and provide facilities and measurements for outside users, while retaining and utilizing our existing expertise in INAA and RNAA.

The Center for Food Safety and Applied Nutrition, U.S. Food and Drug Administration (FDA), directs and maintains a neutron activation analysis (NAA) facility at the NCNR. This facility provides agency-wide analytical support for special investigations and applications research, complementing other analytical techniques used at FDA with instrumental, neutron-capture prompt-gamma, and radiochemical NAA procedures, radioisotope $\mathrm{x}$-ray fluorescence spectrometry (RXRFS), and low-level gamma-ray detection. This combination of analytical techniques enables diverse multielement and radiological information to be obtained for foods and related materials. The NAA facility supports agency quality assurance programs by developing in-house reference materials, by characterizing food-related reference materials with NIST and other agencies, and by verifying analyses for FDA's Total Diet Study Program. Other studies include the development of RXRFS methods for screening foodware for the presence of $\mathrm{Pb}, \mathrm{Cd}$, and other potentially toxic elements, use of instrumental NAA to investigate bromate residues in bread products, and use of prompt-gamma NAA to investigate boron nutrition and its relation to bone strength.

\section{The Neutron Interactions and Dosimetry Group (Physics}

Laboratory) provides measurement services, standards, and fundamental research in support of NIST's mission as it relates to neutron technology and neutron physics. The national and industrial interests served include scientific instrument calibration, electric power production, radiation protection, defense nuclear energy systems, radiation therapy, neutron radiography, and magnetic resonance imaging.
The Group's activities may be represented as three major activities. The first is Fundamental Neutron Physics including magnetic trapping of ultracold neutrons, operation of a neutron interferometry and optics facility, development of neutron spin filters based on laser polarization of ${ }^{3} \mathrm{He}$, measurement of the beta decay lifetime of the neutron, and investigations of other coupling constants and symmetries of the weak interaction. This project involves a large number of collaborators from universities and national laboratories.

The second is Standard Neutron Fields and Applications utilizing both thermal and fast neutron fields for materials dosimetry in nuclear reactor applications and for personnel dosimetry in radiation protection. These neutron fields include thermal neutron beams, "white" and monochromatic cold neutron beams, a thermal-neutroninduced ${ }^{235} \mathrm{U}$ fission neutron field, and ${ }^{252} \mathrm{Cf}$ fission neutron fields, both moderated and unmoderated.

The third is Neutron Cross Section Standards including experimental advancement of the accuracy of neutron cross section standards, as well as evaluation, compilation, and dissemination of these standards.

Several universities have also established long term programs at the NCNR. The University of Maryland is heavily involved in the use of the NCNR, and maintains several researchers at the facility. Johns Hopkins University participates in research programs in solid-state physics and in instrument development at the NCNR. The University of Pennsylvania is working to help develop biological applications of neutron scattering. It is also participating in the second stage construction of the filter analyzer neutron spectrometer, along with the University of California at Santa Barbara, DuPont, Hughes, and Allied Signal. The University of Minnesota participates in two PRTs, the NG-7 $30 \mathrm{~m}$ SANS and the NG-7 reflectometer. The University of Massachusetts also participates in the latter PRT. 
T he reactor operated for 198 full power (20 MW) days during the past year or $54 \%$ of real time that is equivalent to $77 \%$ of the maximum available operating time. Routinely, the reactor is scheduled to operate on a seven week cycle, seven times a year. Each operating cycle includes 38 days on-line and 11 days shutdown for refueling, routine maintenance, and surveillance tests. This year, several major tasks became due and required additional shutdown time. Included among these are two shipments of spent fuel; replacement of the shim arm assemblies which required removal of the entire core and took only one month, half of that previously; and major modification to the refueling system projected to take two months which was completed in five weeks. In addition, corrective maintenance of the thermal shield and the thermal column cooling systems, refurbishing of the existing cooling tower to assure uninterrupted operation for at least two more years, and finally the biennial retraining, re-examination and requalification of all licensed operations personnel were conducted.

The major engineering effort the past year was the design and specification of a completely new plume-abatement cooling tower to be installed adjacent to the existing one. (Figure 1 compares examples of abated and non-abated cooling towers.) As well as being a larger capacity, more effective and more efficient system, the new tower eliminates vapor plumes down to $-12{ }^{\circ} \mathrm{C}$ ambient. Construction of the new tower basin began in late September 2000 and should be completed before the end of the year. Fabrication of the tower is underway and scheduled for completion in the spring of 2001 to be followed by on-site installation, expected to take approximately four months. No reactor shutdown will be required during this period. The reactor will have to be shut down only for final hookup of electrical, controls, and piping connections and for acceptance and performance testing.

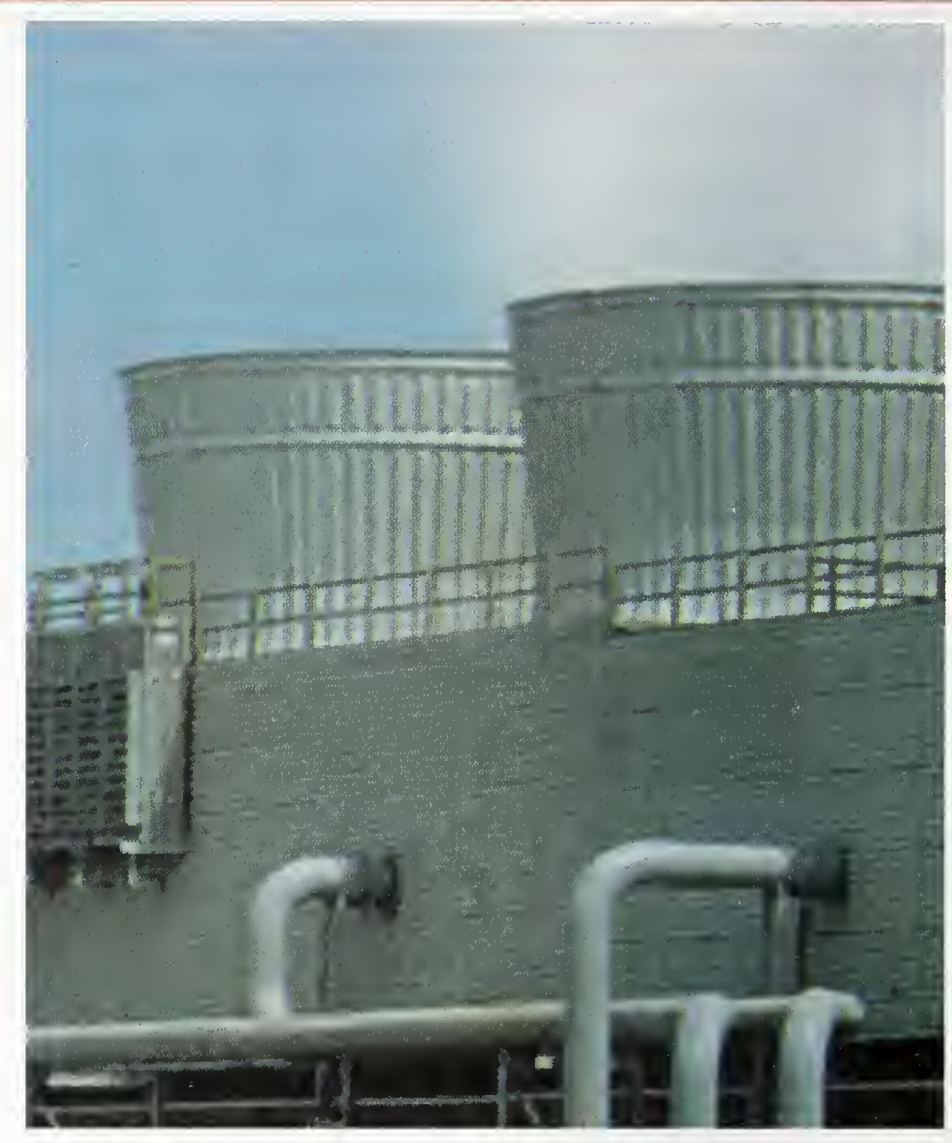

FIGURE 1. No discernible cloud is emitted by the plume-abated cooling tower shown on the left compared to the plumes emerging from the non-abated towers on the right.

Preparations for reactor re-licensing in 2004 for an additional 20 years are proceeding. They include preparation of an updated safety analysis report including seismic evaluation, an environmental report and impact statement, technical specifications and bases, operator requalification program and emergency and security plans. In-service inspections of reactor internals and ultrasonic testing of the primary cooling system plus upgrade of older systems and components will be needed in support of the application for license renewal. Many of the upgrades have already been completed or are in progress. Among the major upgrades planned over the next few years are complete replacement of the nuclear instrumentation panel and associated safety and control systems, complete replacement of the electrical power systems and associated switch gear and replacement and upgrade of the reactor emergency power supply systems. 


\section{A LOW BACKGROUND DOUBLE FOCUSING NEUTRON MONOCHROMATOR}

Work continues on the development of a low background double focusing monochromator which was described in the 1999 NCNR report. The actively controlled double focusing monochromator consists of an array of 315 pyrolytic graphite crystals mounted on 21 thin aluminum blades (see Fig. 1). When buckled, each variable thickness blade conforms in shape to an arc of constant radius providing active vertical focus control. Horizontal focus is accomplished by independently controlling the rotation of each blade.

The design and choice of materials for the system reduces scattering from the supporting structure, a problem common to traditional lead-screw and lever controlled monochromators. Struciural material in the beam is limited to the 21 blades and three thin walled aluminum posts. The 315 crystals are accurately suspended with only $630 \mathrm{~g}$ of structural material in the beam's direct line of sight.

An engineering mock-up of the focusing system was constructed (see Fig. 2a). This three-blade version of the full-scale 21-blade unit was used to study blade performance, develop control software, quantify horizontal and vertical focus performance, and test mechanical and electrical system components. Figure $2 \mathrm{~b}$ shows an optical test of vertical focus performance using the mock-up. The

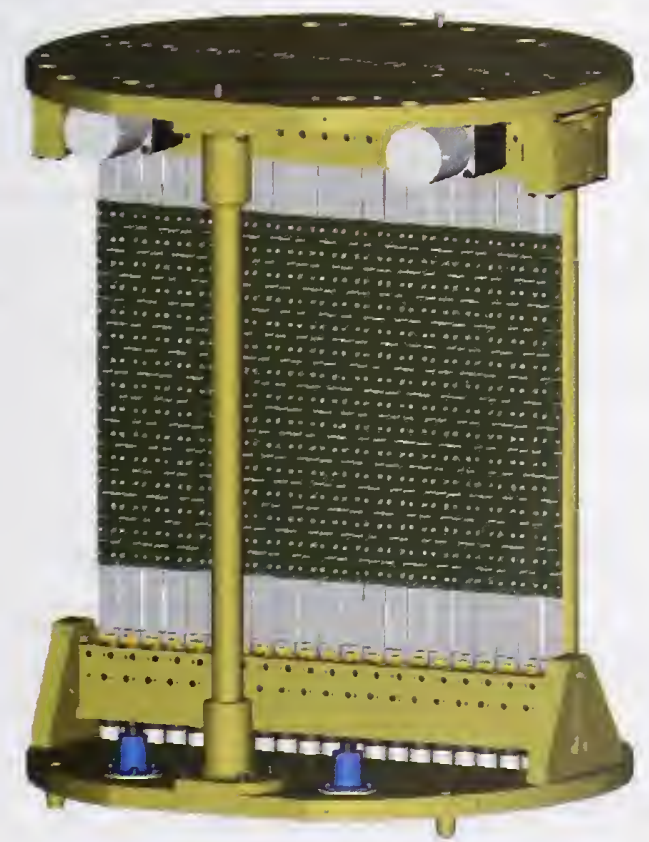

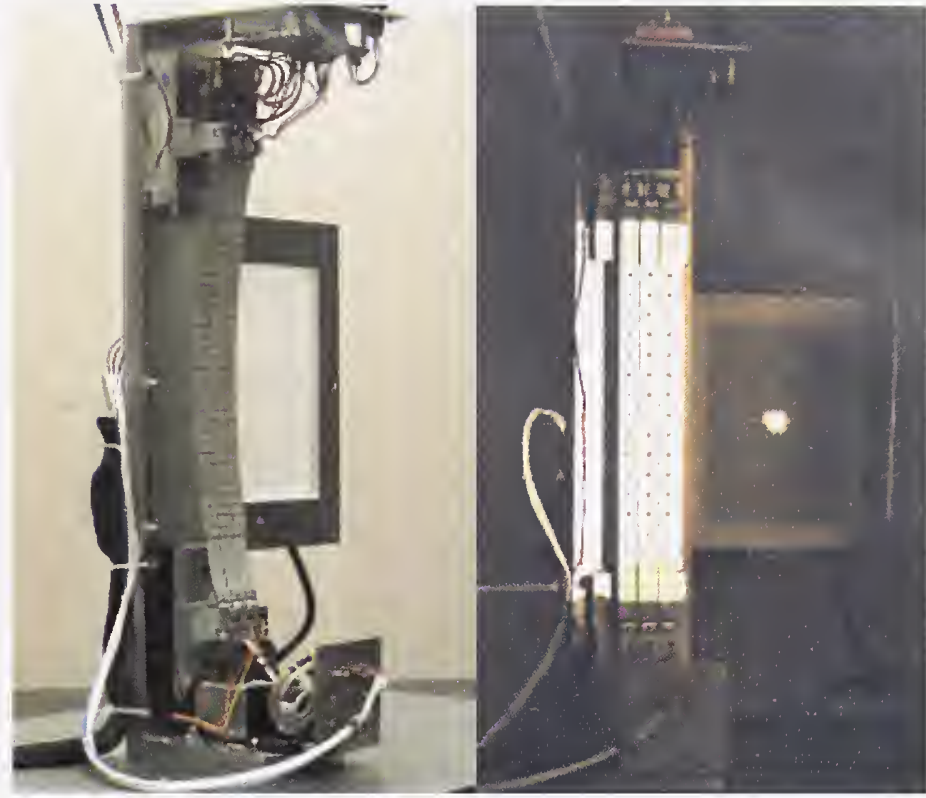

FIGURE 2. Three blade mockup of the double focusing monochromator focusing system. (a) Blades are shown buckled to an arc of a $1 \mathrm{~m}$ radius circle. (b) Vertical focusing is optically verified by focusing a white point source onto a screen.

three blades are covered with reflective mirrors and illuminated with a white point source. The reflected image is focused onto a screen. Imaging tests such as this, as well as mechanical measurements, have verified that errors in blade shape are negligible compared to contributions due to crystal mosaic over the focal range of interest. Similar optical tests have been used to verify the horizontal focus performance.

The full-scale unit is currently under construction. When completed, the $1300 \mathrm{~cm}^{2}$ monochromator will be the heart of the new cold neutron spectrometer under development at the NCNR. It is expected to provide an intense monochromatic neutron flux with 0.1 $<\Delta \mathrm{E}<0.5 \mathrm{meV}$ and $\Delta \mathrm{Q} \approx 0.1 \AA^{-1}$ yielding a peak flux of order 1.0 $\times 10^{8} \mathrm{n} / \mathrm{cm}^{2} / \mathrm{s}$, higher than any currently available worldwide. This new instrument will be ideal for studying materials with excitations having a low characteristic velocity. The enhanced sensitivity will enable inelastic neutron scattering studies of smaller sample size and will provide dynamic information of unprecedented detail when large samples are available. 


\section{THE BT=7 THERMAL TRIPLE AXIS}

\section{ANALYZER/DETECTOR SYSTEM}

As part of the modernization of the thermal neutron spectrometers a new triple-axis instrument is being designed for the BT-7 thermal beam port. For the analyzer portion of this machine several different types of systems have been proposed. One type is a horizontally focused pyrolytic graphite analyzer system shown in Fig. 3. The analyzer crystal system consists of 13 pyrolytic graphite blades, each $2 \mathrm{~cm}$ wide and $15 \mathrm{~cm}$ high, with either an individual detector for each of the 13 blades, or a position-sensitive detector using all the blades at once.

This is the modern equivalent of our present analyzer systems, and is expected to be the workhorse for the new thermal triple axis instruments. The blades of the analyzer can be freely rotated by 360 degrees and individually positioned, while the entire unit can be rotated as a whole to achieve the desired focusing condition. Each blade can then be matched with a detector that is capable of being positioned individually by a stepper motor on a circular track around the analyzers. A straight-through beam monitor is incorporated into the shielding behind the analyzer crystals to continuously monitor the flux of neutrons entering the analyzer system. A separate diffraction detector is also provided, which can be moved in front of the analyzer if the energy-integrated signal is to be measured.

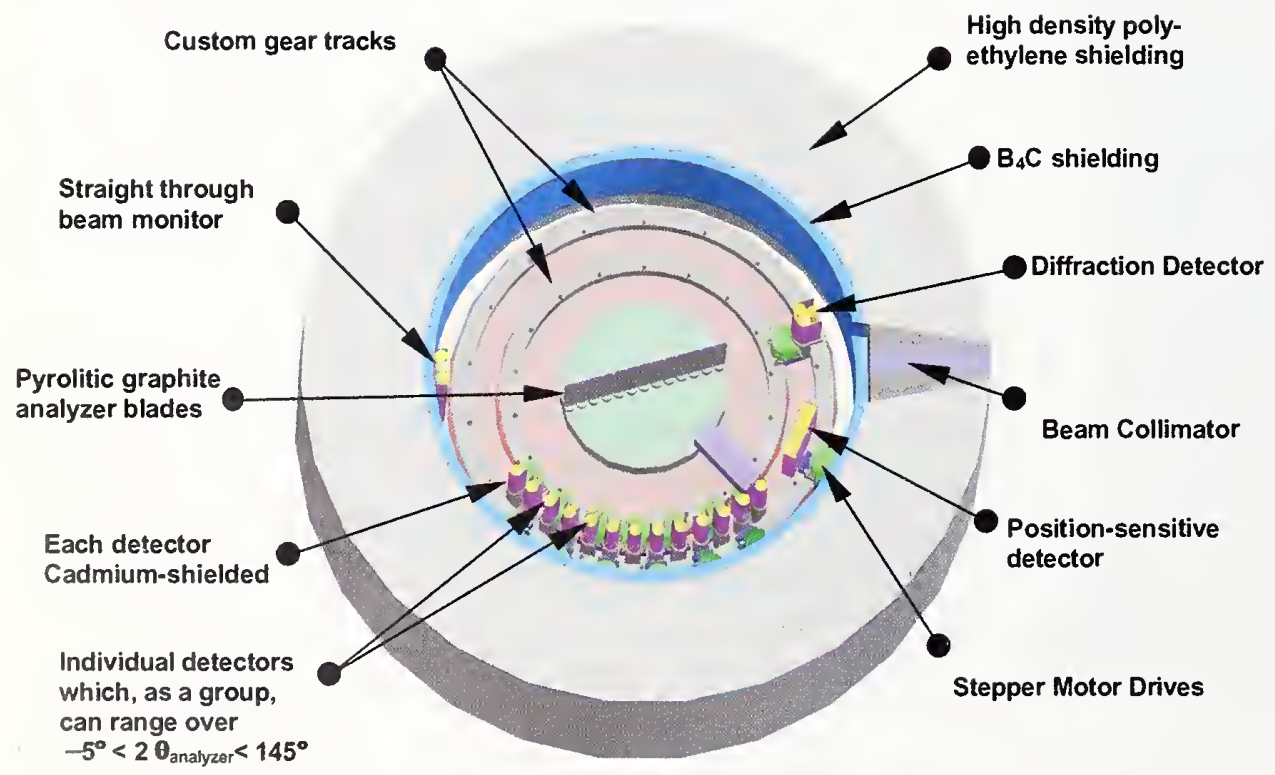

The general design philosophy is to make the instrument as user friendly as possible while still meeting all the desired operating criteria. These include a built-in magnetic guide field for polarized beam operation; and various beam defining systems such as collimators. beam apertures, spin flippers, and filters. Ease of exchanging beam collimators before and after the analyzer crystals is an important design feature that presents an engineering challenge. Extracting the wiring from all the moving detectors and motors inside the system will also be a technical challenge.

A second type of analyzer system will consist of a series of up to 30 individual and isolated analyzer/detector systems. Other analyzer options, to be developed in the future, include incorporating a velocity selector into the analyzer system, and developing a "conventional" double-focusing analyzer with a single, well-shielded detector.

\section{THERMAL NEUTRON PROMPT GAMMA-RAY ACTIVATION ANALYSIS (PGAA) FACILITY AT VT.5.}

The vertical beam tube VT-5 thermal neutron PGAA facility is being upgraded through a collaboration of members of the NCNR, the Nuclear Methods Group, and the U.S. Food and Drug Administration. The current facility consists of an internal neutron-collimating beam tube and shutter assembly, an external beam tube, sample chamber, a beam stop, and a gamma-ray detection system. All components except the internal beam tube and shutter assembly will be replaced and a sapphire filter installed in the shutter assembly. The new components will be designed to reduce background count rates and improve detection limits. The external components will be constructed as a single unit to simplify removal and re-assembly of the instrument to make room for reactor refueling. 


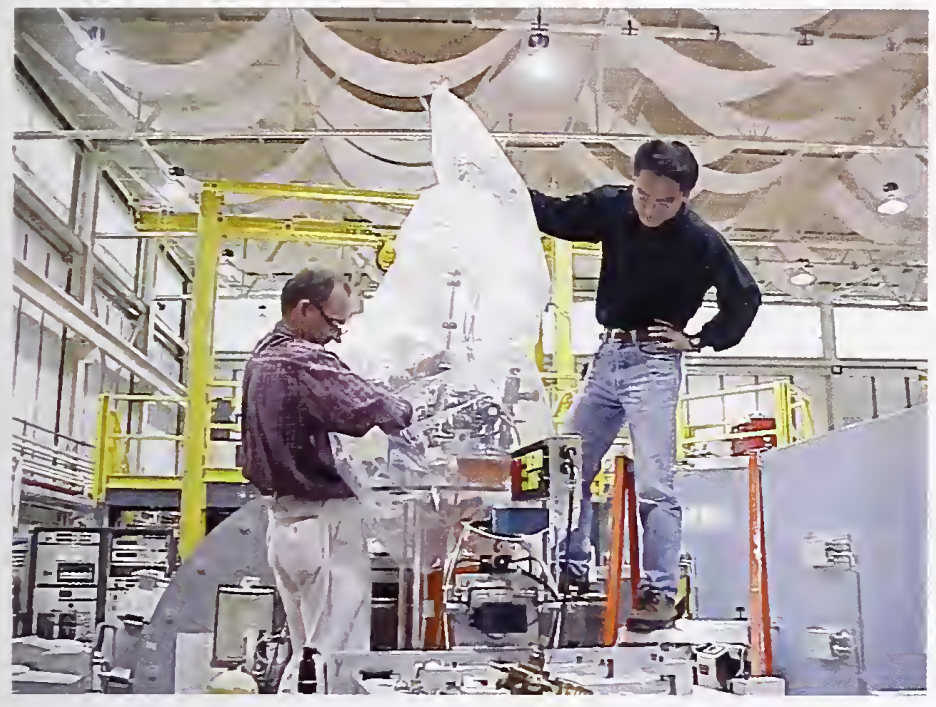

FIGURE 4. Andrzej Rajca (left, University of Nebraska) and NCNR's Sung-Min Choi load a sample under special conditions. For this SANS experiment, the sample had to be kept below $170 \mathrm{~K}$ from storage in liquid nitrogen to toploading into the pre-cooled cryostat. The transfer was performed in a helium atmosphere using a glove bag in order to prevent water condensation on the sample cell surface.

A cylindrical sapphire filter $5.3 \mathrm{~cm}$ long $(4.3 \mathrm{~cm}$ diameter $)$ was added to the shutter. This modification reduced the number of fast neutrons by a factor of five and greatly reduced the low-energy gamma-ray background. The gamma-ray background measured at the perimeter of the apparatus has decreased by a factor of two and the peak-to-background ratios for gamma-ray energies below 500 $\mathrm{keV}$ increased by a factor of two to three.

The design of the new system is complete. The steel component of the old beam stop will be replaced by aluminum to eliminate iron capture gamma-ray background. The external beam tube will be evacuated to minimize neutron scattering and capture in air and will be constructed from aluminum tubing lined internally with lithoflex to eliminate the background for boron determinations. The detection system will consist of a $40 \%$ (relative efficiency) germanium detector with a bismuth germanate Compton suppression system. The new system will decrease analysis times and improve detection for most elements determined.

\section{SAMPLE ENVIRONMENT TEAM}

Fiscal year 2000 marked the creation of a new Sample Environment Team, dedicated to providing a central resource for the sample environment needs of neutron researchers. This team provides a single contact point to resolve any issue related to the sample condi- tions during an experiment, such as temperature, pressure, magnetic field, electric field, or in situ measurements. The scope of this team includes sample preparation and mounting, equipment scheduling, special equipment configurations, operational procedures, provision of necessary resources, sample storage, and equipment repair. Figure 4 shows researchers loading a sample. In this case a difficult SANS experiment was made possible by the team working with the researchers to choose the appropriate sample environment equipment and operating procedures.

The most visible change during FY2000 is in the high-bay sample preparation area at the NCNR. This area was extensively remodeled to provide more workspace, greater ease of use, more convenient preparation of cryogenic equipment, and better access to sample storage. Additionally, extensive documentation is now available for the most widely used sample environment equipment, the closed-cycle helium refrigerators. This documentation details individually-measured operating characteristics, sample mounting information, temperature sensor data, and operating guidelines. Significant new equipment acquired for the user community in FY2000 is listed in Table 1.

TABLE 1. Significant sample environment purchases during FY2000.

\begin{tabular}{ll}
$\begin{array}{l}100 \mathrm{~mm} \text { access top-loading } \\
\text { helium cryostat }\end{array}$ & $\begin{array}{l}1.5 \mathrm{~K} \text { to } 300 \mathrm{~K} \text {, dedicated to } \\
\text { time-of-flight spectrometer }\end{array}$ \\
\hline $\begin{array}{l}70 \mathrm{~mm} \text { access top-loading } \\
\text { helium cryostat }\end{array}$ & $1.5 \mathrm{~K}$ to $300 \mathrm{~K}$, general use \\
\hline $\begin{array}{l}\text { Seven additional sample } \\
\text { probes for } 50 \mathrm{~mm}, 70 \mathrm{~mm} \text {, and } \\
100 \mathrm{~mm} \text { access top-loading } \\
\text { helium cryostats }\end{array}$ & $\begin{array}{l}\text { Provide for faster sample changes } \\
\text { and specialized needs such as } \\
\text { gas handling }\end{array}$ \\
\hline Closed-cycle helium refrigerator & $\begin{array}{l}10 \mathrm{~K} \text { to } 350 \mathrm{~K} \text {, dedicated to } \\
\text { backscattering spectrometer }\end{array}$ \\
\hline $\begin{array}{l}\text { Closed-cycle helium refrigerator } \\
\text { Electromagnet }\end{array}$ & $10 \mathrm{~K}$ to $350 \mathrm{~K}$, general use \\
\hline $\begin{array}{l}\text { Thirty indium-sealing } \\
\text { sample cans }\end{array}$ & $\begin{array}{l}0-0.7 \text { Tesla, dedicated to } \\
\text { vertical reflectometer }\end{array}$ \\
\hline Six temperature controllers & $\begin{array}{l}\text { Mounting of powder samples and } \\
\text { single crystal samples }\end{array}$ \\
\hline Nine turbopumps & $\begin{array}{l}\text { Multiple sensor capability, } \\
\text { PID control }\end{array}$ \\
\hline Two large rotary vane pumps & $10^{-7}$ mbar base pressure \\
\hline
\end{tabular}




\section{MATERIALS SCIENCE/CRYSTALLOGRAPHY}

Ab-Initio Structure Solution of Complex Oxides Using Neutron and X-ray Powder Diffraction Data

P. Woodward ${ }^{129}$ and P. Underwood 129

A Bond-Valence Analysis of the Structure of $\mathrm{YBa}_{2} \mathrm{Fe}_{3} \mathrm{O}_{8}$

I. Natali Sora ${ }^{173}$ and A. Santoro ${ }^{105}$

Aerogels Investigated by SANS with Contrast Masking Solutions C. Merzbacher ${ }^{101}$, M. Anderson ${ }^{101}$, V. Cepak' ${ }^{101}$, and J. G. Barker ${ }^{105}$

Cation Ordering in Magnetically Frustrated Pyrochlores

M. A. Green ${ }^{147}$, T. Fenne||147, and S. Bramwel|147

Characterization of $\mathrm{Ag}$ and Mixed $\mathrm{Li} / \mathrm{Ag}$ Faujasite Zeolites Using

Neutron Diffraction

N. Hutson ${ }^{202}$, R. Yang 202, B. A. Reisner ${ }^{105}$, and B. H. Toby ${ }^{105}$

Chemical Ordering in Ni-Mn-Ga Heusler Alloys

R. Overholser ${ }^{199}$, M. Wuttig ${ }^{199}$, and D. A. Neumann ${ }^{105}$

Comparison of Residual Stress Measurement Techniques

T. Gnäupel-Herold 199,105, P. C. Brand ${ }^{105}$, H. J. Prask ${ }^{105}$, S. Spooner ${ }^{127}$, B.

Pardue ${ }^{161}$, D. Nelson ${ }^{156}$, and S. Foss ${ }^{70}$

'CONV' and 'STRESS': New Codes for Data Reduction in Neutron

Diffraction Residual Stress Measurements

P. C. Brand ${ }^{105}$ and T. Gnäupel-Herold 199,105

Crystal Chemistry of Protonic Centers in Zeolites

D. H. Olson ${ }^{210}$ and B. H. Toby ${ }^{105}$

Crystal Structure of $\mathrm{Ba}_{4} \mathrm{CaCu}_{2} \mathrm{O}_{6}\left(\mathrm{CO}_{3}\right)$

Q. Z. Huang 199,105 and F. Izumi95

Crystal Structure of Dehydrated LaD-LSX

D. H. Olson ${ }^{210}$, B. A. Reisner ${ }^{105}$, and B. H. Toby ${ }^{105}$

Crystal Structure Determinations of the $\mathrm{CeMnO}_{3}$ Compound

W. C. Li94

Defect Structure of $(\mathrm{Cu}, \mathrm{Zn}) \mathrm{InSe} \mathrm{S}_{2}$

B. A. Reisner ${ }^{105}$, M. Knox ${ }^{178}$, A. Stacy ${ }^{178}$, and G. Ron ${ }^{178}$

The Development of Capabilities for the Determination of Residual

Stress on the Mesoscale

H. J. Prask ${ }^{105}$, P. C. Brand ${ }^{105}$, T. Gnäupel-Herold ${ }^{199,105}$, D. Balzar ${ }^{112}$, D. Hurley ${ }^{112}$, and G. Alers ${ }^{112}$

Development of a Standard for Residual Stress Measurements as Part of VAMAS

T. Gnäupel-Herold 199 , 105, P. C. Brand ${ }^{105}$, H. J. Prask ${ }^{105}$, and G. A. Wester ${ }^{56}$

Diffraction Elastic Constants for Arbitrary Specimen and Crystal

Symmetries: Theory and Practical Consequences

T. Gnäupel-Herold ${ }^{199,} 105$ and H. J. Prask ${ }^{105}$

Distribution of Hydrogen in $\mathrm{LaNi}_{5}$ and $\mathrm{LaNi}_{5-\mathrm{x}} \mathrm{Sn}_{\mathrm{x}}$

B. Fultz' ${ }^{18}$, C. K. Witham ${ }^{18}$, T. Udovic ${ }^{105}$, and R. Bowman ${ }^{18}$

Effect of Annealing on $\mathrm{ZrO}_{2}$ Phase Composition

J. K. Stalick ${ }^{105}$ and J. Ilavsky29

The Effect of Texture on Elastic Constants

H. Sitepu ${ }^{157}, 105,148$, T. Gnäupel-Herold ${ }^{199}{ }^{105}$, J. Matejicek ${ }^{64}$, R. Fields ${ }^{113}$, and H. J. Prask ${ }^{105}$

The Effect of Texture on Rietveld Refinement of Powder Diffraction

Data of Molybdite and Calcite

H. Sitepu157.105.148, T. Gnäupel-Herold ${ }^{199,105}$, and H. J. Prask ${ }^{105}$

Ethylene Sorption by Copper Aluminum Chloride

R. Sullivan ${ }^{121}$, J. Martin ${ }^{121}$, H. Liu'157, and C. Grey'157

Exploration of $\mathrm{Fe}$ Disproportionation in $\mathrm{Ba}_{3} \mathrm{FeO}_{5}$

B. A. Reisner ${ }^{105}$, J. Delattre ${ }^{178}$, and A. Stacy ${ }^{178}$

Formation of Titanium Mesoporous Materials

P. D. Butler ${ }^{105,} 127$
Grazing Incidence SANS From Plasma Sprayed Coatings

A. J. Allen ${ }^{109}$ and H. Boukari109

Hybridized Epoxy-Silicate Nanocomposites

N. Beck-Tan ${ }^{6}$, S. Young ${ }^{6}$, and S. F. Trevino ${ }^{9}, 105$

Internally Oxidized Palladium Alloys: Particle Size Determination by

SANS

J. G. Barker ${ }^{105}$ and T. B. Flanagan 221

Investigation of the $\mathrm{Zr}_{9} \mathrm{Pt}_{11}$ Structure Type

J. K. Stalick ${ }^{105}$ and R. Waterstrat' ${ }^{105}$

In Situ Neutron Reflectivity Measurements of Deuterium Absorption in

$\mathrm{Pd}$ Films

B. J. Heuser ${ }^{195}$, A. Munter ${ }^{105}$, N. Barber ${ }^{195}$, J. A. Dura ${ }^{105}$, and C. F. Majkrzak ${ }^{105}$

Magnetic Cluster Sizes in Thin Film Magnetic Recording Disks

M. Toney ${ }^{55}$, S. M. Choi 199.105 , and C. J. Glinka105

Magnetism in Layered Manganates

M. A. Green ${ }^{147}$

Measurement and Modeling of Residual Stresses in Multi-Pass

Weldments

T. Gnäupel-Herold 199,105, H. J. Prask ${ }^{105}$, R. Lloyd ${ }^{56}$, and W. Reuter ${ }^{56}$

Microstructure of Alkali Activated Amorphous Binders

A. J. Allen ${ }^{109}$ and J. Phair217

Microstructure Evolution of Fly Ash Blended Cements

A. J. Allen ${ }^{109}$ and R. A. Livingston ${ }^{39}$, and W. Bumrongiaroen ${ }^{39}$

Morphological and Kinetic Study of Early Calcium-Silicate-Hydrate Gel

Development During the Hydration of Cement and Tricalcium Silicate H. M. Jennings ${ }^{122}$, J. J. Thomas ${ }^{122}$, and A. J. Allen ${ }^{109}$

Negative Thermal Expansion in $\mathrm{AOPO}_{4}$ Compounds

A. W. Sleight ${ }^{132}$ and T. Amos ${ }^{132}$

Neutron Diffraction of Heusler Alloys

V. G. Harris ${ }^{101}$ and Q. Z. Huang ${ }^{199,105}$

Neutron Diffraction of Single-Wall Carbon Nanotubes

P. Papanek210, 105, J. E. Fischer ${ }^{210}$, Z. Benes ${ }^{210}$, D. Colbert ${ }^{145}$, and M. Heben ${ }^{145}$

Neutron Diffraction Study of Copper(I)-Zirconium(IV) Chlorides

A. Dattelbaum" ${ }^{121}$, J. Martin ${ }^{121}$, and B. A. Reisner ${ }^{105}$

A New Double-Focusing Monochromator for "DARTS"

T. Gnäupel-Herold199. 105, H. J. Prask ${ }^{105}$, and M. Popovici204

Order-Disorder in CoFe/Carbon Composites

J. H. Scott ${ }^{119}$, M. E. McHenry ${ }^{19}$, Z. Turgut ${ }^{19}$, and M. Storch ${ }^{19}$

Phase Analysis of Sintered Yttria-Stabilized Zirconia

J. K. Stalick ${ }^{105}$, A. Slifkaa ${ }^{112}$, and D. Balzar ${ }^{112}$

Phase Composition Analysis of Plasma-Sprayed Partially Stabilized

Zirconia Coatings by Neutron Powder Diffraction Data

H. Sitepu157. 148, 105, H. J. Prask ${ }^{105}$, J. K. Stalick ${ }^{105}$, R. Goswani157, and S.

Sampath ${ }^{157}$

Polymerized Fullerenes

S. F. Trevino. ${ }^{105}$, N. Beck-Tan ${ }^{6}$, and L. Balogh ${ }^{89}$

Pore Structure in Novel Ultra-Low Dielectric Constant Thin Films S. Yang ${ }^{82}$ and E. K. Lin ${ }^{115}$

Residual Stress Determination by Whole Pattern Analysis-Application to Synchrotron HEXRD Measurements on Thermal Sprayed Coatings T. Gnäupel-Herold ${ }^{199}$, 105, D. R. Haeffner ${ }^{4}$, H. J. Prask ${ }^{105}$, and J. Matejicek ${ }^{64}$

Residual Stress Determination for Welds in Navy-Ship Steel T. Gnäupel-Herold ${ }^{199}, 105$, H. J. Prask ${ }^{105}$, and X. Cheng ${ }^{78}$

Residual Stresses in Thermally-Sprayed Coatings J. Matejicek ${ }^{64}$, T. Gnäupel-Herold 199,105, P. C. Brand ${ }^{105}$, H. J. Prask ${ }^{105}$, H. Sitepu ${ }^{157,148,105}$, and S. Sampath 157 
Residual Stress in Thermally Sprayed Components for Automotive

Applications

J. Matejicek64, D. Wilkosz'43, T. Gnäupel-Herold199, 105, H. J. Prask'105,

H. Sitepu157. 148, 105, and S. Sampath ${ }^{157}$

Resolving Cationic Sittings in a new Dielectric Phase:

$\mathrm{Bi}_{1.5} \mathrm{Zn}_{0.5} \mathrm{Ti}_{1.5} \mathrm{Nb}_{0.5} \mathrm{O}_{7}$

B. A. Reisner ${ }^{105}$, and J. Chan ${ }^{109}$

A Ring/Plug Reference Specimen for Residual Stress Measurements T. Gnäupel-Herold199. 105, C. S. Hehman77, 112, N. Nguyen ${ }^{47.112, ~ A . ~ V . ~ C l a r k ' 158, ~}$ and H. J. Prask 105

The Role of Water in Natural Disordered Opal-CT Samples D. Bish ${ }^{80}$ and G. Guthrie80

SANS Characterization of Hydrides in Uranium S. Spooner ${ }^{127}$, G. Ludtka127, and J. G. Barker ${ }^{105}$

SANS Characterization of Low Dielectric Constant Polymer Nanoporous Thin Films W.-L. Wu'15, E. K.Lin ${ }^{115}$ B. Landes ${ }^{32}$, and G. Barnes ${ }^{32}$

SANS of Hardened Cement Paste and Nanoporous Reference Materials at Different Relative Humidifies J. Adolphs' 122 and J. J. Thomas ${ }^{122}$

SANS From Lithographically Patterned Polymer Thin Films W.-L. Wu'115, E. K. Lin ${ }^{115}$, N. F. Berk105, and C. J. Glinka105

SANS Study of Anisotropic Microstructure in Plasma-Spray Coatings H. Herman ${ }^{157}$, A. Kulkarni157, A. J. Allen ${ }^{105}$, and G. G. Long ${ }^{105}$

SANS Study of Deuteride Formation and Decomposition in Palladium B. J. Heuser ${ }^{195}$ and W. C. Chen ${ }^{195}$

SANS Study of Fire Retardant Polymer-Clay Nanocomposites H. J. M. Hanley'120, C. D. Muzny ${ }^{120}$, and J. Gilman ${ }^{127}$

SANS Study of Nucleation of Aluminum Hydroxide J. Watson', R. Knott', and M. Y. Lin ${ }^{37.105}$

SANS and SAXS Determination of the Dispersion in Organic Solvents of Organically Modified Clays for Polymer/Clay Nanocomposites D. Ho ${ }^{199}$, R. M. Briber ${ }^{199}$, and C. J. Glinka ${ }^{105}$

$\mathrm{SiO}_{2}$ Ultra Thin Films Round Robin J. A. Dura105, C. F. Majkrzak'105, and J. Ehrstein ${ }^{116}$

Site Distributions in the Structure of $\mathrm{Ba}_{2} \mathrm{Sn}_{2} \mathrm{ZnGa}_{10-\mathrm{x}} \mathrm{Cr}_{\mathrm{x}} \mathrm{O}_{22}(\mathrm{x}=3$ and 4) Q. Z. Huang ${ }^{199},{ }^{105}$, R. Cava ${ }^{140}$, I. S. Hagemann ${ }^{140}$, X. P. A. Gao ${ }^{82}$, and A. P. Ramirez ${ }^{82}$

Size Distribution of Titania Particles in Coatings L.-P Sung ${ }^{108}$ and D. Ho ${ }^{199 .} 105$

Small-Angle Magnetic Scattering From Nanocrystalline Cobalt J. Weismüller ${ }^{174}$, R. D. Shull ${ }^{109}$, R. McMichael ${ }^{109}$, U. Erb ${ }^{142}$, and J. G. Barker ${ }^{105}$

Solute Effects on Membrane Thickness in DOPG and DOPC J. Pencer ${ }^{194}$ and R. Hallett 194

Structural Distortions in the $\mathrm{NaSr}_{3} \mathrm{MO}_{6}$ ( $\mathrm{M}=\mathrm{Ru}, \mathrm{Rh}$ ) Family B. A. Reisner ${ }^{105}$, A. Prieto ${ }^{178}$, and A. Stacy ${ }^{178}$

Structural Distortions in Titanites B. A. Reisner ${ }^{105}$ and D. Xirouchakis ${ }^{93}$

Structural Investigations of ETS-4 Microporous Molecular Sieves M. Tsapatsis 201 , H. Hillhouse ${ }^{201}$, R. Jacubinas ${ }^{38}$, and B. Toby ${ }^{105}$

Structural Ordering in Erbium Doped $\mathrm{Bi}_{2} \mathrm{O}_{3}$ B. A. Reisner ${ }^{105}$, E. Wachsman191, T. J. Udovic ${ }^{105}$, and D. A. Neumann ${ }^{105}$

Structural Refinement of Mixed-Metal Vanadium Phosphates P. DeSanto, Jr. ${ }^{190}$ and D. Buttrey ${ }^{190}$

Structural Studies on High Dielectric Materials R. Harlow ${ }^{33}$ and M. Subramanian ${ }^{33}$

Structural Study of $\mathrm{Sr}_{4} \mathrm{Nb}_{2} \mathrm{O}_{9}$ Compound I. Levin ${ }^{109}$ J. Chan ${ }^{109}$, and T. Vanderah ${ }^{109}$
Structural Studies of Strontium Niobium Oxides

W. Wong-Ng ${ }^{109}$, W. Greenwood ${ }^{199}$, and B. H. Toby ${ }^{105}$

Structure of Barium Titanate Thin Films on Silicon

C. Bouldin ${ }^{109}$, B. Revel109, and J. A. Borchers ${ }^{105}$

Structure, Cation Ordering and Magnetism in Manganate Spinels M. A. Green ${ }^{147}$ and D. A. Neumann 105

Structure of $\mathrm{Ca}_{2} \mathrm{AlNbO}_{6}$ and $\mathrm{CaTiO}_{3}$ Compounds

I. Levin ${ }^{109}$, J. Chan ${ }^{109}$, and T. Vanderah ${ }^{109}$

Structure Determination of $(\mathrm{Ca}, \mathrm{Sr})_{2}(\mathrm{Rh} / \mathrm{r}) \mathrm{D}_{5}$ by Powder Neutron

Diffraction R. O. Moyer ${ }^{171}$ and B. H. Toby ${ }^{105}$

Structure Determination of Framework Materials with the RHO

Topology B. A. Reisner ${ }^{105}$

Structure Determination of $\mathrm{La}_{0.78} \mathrm{Sr}_{0.22} \mathrm{RuO}_{3}$ Q. Z. Huang ${ }^{199}, 105$, I. Natali Sora ${ }^{173}$, A. Santoro ${ }^{105}$, and F. T. Vanderah ${ }^{109}$

Structure of the Layered Cobalt Oxychlorides $\mathrm{Sr}_{3} \mathrm{Co}_{2} \mathrm{O}_{5} \mathrm{Cl}_{2}$ and

$\mathrm{Sr}_{2} \mathrm{COO}_{3} \mathrm{Cl}$

Q. Z. Huang ${ }^{199},{ }^{105}$, N. McGlothlin ${ }^{140}$, T. He ${ }^{140}$, and R. J. Cava ${ }^{140}$

Structure of the $n=2$ and $n=\infty$ Member of the Ruddlesden-Popper

Series, $\mathrm{Sr}_{n+1} \mathrm{Sn}_{n} \mathrm{O}_{3 \mathrm{n}+1}$

M. A. Green ${ }^{147}$, K. Prassides ${ }^{216}$, P. Day ${ }^{147}$, and D. A. Neumann ${ }^{105}$

Structure and Oxygen Disorder in $\mathrm{Sr}_{1.9} \mathrm{Nd}_{1.1} \mathrm{Cu}_{2} \mathrm{O}_{\mathrm{x}}$ Q. Z. Huang ${ }^{199}, 105$ and W. Wong-Ng109

Structure of Porous Manganese Oxides M. A. Green ${ }^{147}$, D. Arnold ${ }^{147}$, and A. Lappas ${ }^{147}$

Structure of Porous Silica Thin Films W.-L. Wu'115 and E. K. Lin ${ }^{115}$

Structure Refinements of Alumo- and Gallo-Germanates Using Neutron Scattering Techniques S. Park ${ }^{157}$, J. B. Parise ${ }^{157}$, and B. H. Toby ${ }^{105}$

Structure Refinement of a Ferromagnetic Vanadium(III) Sulfate D. Grohol ${ }^{83}$, D. Nocera ${ }^{83}$, B. H. Toby ${ }^{105}$, and Q. Z. Huang ${ }^{199}, 105$

Structure of $75.25: 24.75 \mathrm{CaO}-\mathrm{Nb}_{2} \mathrm{O}_{5}$ J. Chan ${ }^{109}$ I. Levin ${ }^{109}$, R. Roth ${ }^{109}$, T. Vanderah ${ }^{109}$, and B. A. Reisner ${ }^{105}$

Structure of $\mathrm{Sr}_{1.875} \mathrm{Dy}_{1.125} \mathrm{Cu}_{2} \mathrm{O}_{\mathrm{x}}$ Q. Z. Huang 199,105 and W. Wong-Ng ${ }^{109}$

Structures of $\mathrm{Ba}_{0.8} \mathrm{Sr}_{0.2} \mathrm{CoO}_{y}$ Compounds

Q. Z. Huang ${ }^{199.105}$ and R. J. Cava ${ }^{140}$

Studies of Charge, Spin and Orbital Ordering in the System

$\mathrm{Bi}_{1-x} \mathrm{Ca}_{x} \mathrm{MnO}_{3}$ P. Woodward ${ }^{132}$, J. Goldberger ${ }^{132}$ and P. N. Santosh ${ }^{132}$

Synthesis, Structure and Electronic Properties of $\mathrm{LaCa}_{2} \mathrm{Mn}_{2} \mathrm{O}_{7}$ M. A. Green ${ }^{147}$ and D.A. Neumann ${ }^{105}$

Thermal Expansion of Alpha SiC From 15 to $298 \mathrm{~K}$ R. Reeber ${ }^{121}, 7$, K. Wang ${ }^{121}$, and B. H. Toby' ${ }^{105}$

Thermal Expansion of $\mathrm{HgSe}$ R. Reeber ${ }^{121,7}$ and K. Wang ${ }^{12}$

Thermal Expansion of II-VI Semiconductors R. Reeber ${ }^{121,7}$ and K. Wang ${ }^{121}$

Texture Characterization in X-ray and Neutron Powder Diffraction Data Using the Generalized Spherical-Harmonic H. Sitepu ${ }^{157}, 105,148$, H. J. Prask ${ }^{105}$, and M. D. Vaudin ${ }^{109}$

Texture Determination of $\left(\mathrm{CF}_{2}\right)_{n}$ Films Using Neutron Diffraction H. Sitepu157, 105, 148, T. Gnäupel-Herold ${ }^{199}, 105$, J. D. Barnes ${ }^{115}$, and H. J. Prask ${ }^{105}$

Texture Determination of NAFION Using Neutron Diffraction H. Sitepu $157,105,148$, T. Gnäupel-Herold ${ }^{199,}{ }^{105}$, S. F. Trevino9. 105, and H. J. Prask ${ }^{105}$ 
Texture in Plasma-Sprayed Coatings

H. Sitepu 157, 105, 148, T. Gnäupel-Herold199, 105, S. Sampath ${ }^{157}$, and H. J. Prask'105

Texture and Residual Stresses in Hydroformed Aluminum Tubing

T. Gnäupel-Herold199, 105, H. J. Prask ${ }^{105}$, and K. N. Shah ${ }^{3}$

\section{POLYMERS}

Aggregation of Fullerene-Based Star-lonomers

T.-L. Lin ${ }^{100}, J$. U-Ser'100, and L. Wen Jiun'100

Behavior of Polymer Chains in Porous Media

M. P. Nieh ${ }^{137}$, S. K. Kumar ${ }^{137}$, R. M. Briber ${ }^{199}$, and L.-T. Ho ${ }^{199}, 105$

Chair Dimensions of Captured Micelle Star Polymers

L. Fetters ${ }^{37}$, M. Xenidou ${ }^{37}$, M. Y. Lin ${ }^{37}, 105$, and N. Balsara'184

Characterization of Dye Distribution in Polymer Thin Films

C. White ${ }^{115}$, E. K. Lin ${ }^{115}$, and W.-L. Wu115

Characterization of Organically Modified Clays

L.-T. Ho ${ }^{199}, 105$, R. M. Briber ${ }^{199}$, and C. J. Glinka ${ }^{105}$

Compatibility of Polyelastomers

D. Peiffer' ${ }^{37}$, M. Y. Lin 37,105 , and Y. Zhang 157

Critical Neutron Reflection Tests of Polymer Interdiffusion Dynamics

R. P. Wool ${ }^{190}$, K. A. Welp 190 , and W. Dozier 190

Dendrimer Like Vesicles

S. F. Trevino9, 105

Dynamics of Polymer Interdiffusion

S. K. Satija ${ }^{105}$

Effects of Clay on the Phase Behavior of Polystyrene/PVME Blends

R. Krishnamoorti ${ }^{194}$, K. Yurekli194, and A. Karim ${ }^{115}$

Effects of Substrate Interactions on the Ordering of Block Copolymer

Films

E. Huang ${ }^{201}$, J. DeRouchey201, and T. P. Russel|201

Effects of Supercritical $\mathrm{CO}_{2}$ on the Phase Behavior of Block Copolymer

Melts

J. Watkins ${ }^{201}$, M. Pollard ${ }^{201}$, G. Brown ${ }^{201}$, S. Kumar ${ }^{137}$, and T. P. Russell201

Electric Field-Induced Orientation of Diblock Copolymers in Thin Films

J. DeRouchey201 and T. P. Russell201

Hybrid Nanostructures With Hydrophobically Modified Dendrimers

F. Groehn $n^{115}$ and B. J. Bauer ${ }^{115}$

Inelastic Neutron Scattering From Filled Elastomers

A. I. Nakatani15, R. D. Ivkov'105, P. Papanek210, 105, H. Yang ${ }^{154}$, and M.

Gerspacher 154

Interdiffusion of Cross Linked Polymer Films

U. Perez-Salas ${ }^{199}$, R. M. Birber ${ }^{199}$, M. Rafailovich ${ }^{157}$, J. Sokolov ${ }^{157}$, and N. F. Berk ${ }^{105}$

Investigation of the Concentration Profile Between Cross Linked Thin

Films

R. M. Briber ${ }^{199}$, U. A. Perez-Salas ${ }^{199}$, M. Rafailovich ${ }^{157}$, and J. Sokolov'157

In Situ Study of Molecular Conformation in

Elastomeric Polypropylene in Uniaxial Extension

A. Gast ${ }^{156}$ and J. Pople 156

Liquid Crystal Polymers in a Magnetic Field

M. Dadmun ${ }^{218}$, W. Burghardt'124, and N. Vaish ${ }^{4}$

Low Energy Excitations in Deuterated Polystyrene

R. M. Dimeo ${ }^{105}$ and J. F. Douglas ${ }^{115}$

Melt Blended Polymer-Clay Nanocomposites

H. King 37 , A. Herhold 37 , and M. Y. Lin ${ }^{37}, 105$

Membrane Mediated Polymer Interdiffusion

H. Gruell|115, A. Esker ${ }^{115}$, and C. C. Han ${ }^{115}$.

Microstructure and Properties of Hyperbranched Polymers

N. Wagner ${ }^{190}$ and Y. Kim ${ }^{34}$

Morphological Study of Comb Copolymer Pervaporation Membranes

A. Mayes ${ }^{83}$, A. Akthakuls3, and Y. Y. Won ${ }^{83}$

Multi-Dendrimer Nanostructures

F. Groehn ${ }^{115}$, E. Amis ${ }^{115}$, and B. J. Bauer ${ }^{115}$
Neutron Reflectivity From the Monolayers of SAN Random Copolymers

E. Kim³, H. Kim777, K. Char153, and S. -H. Lee ${ }^{153}, 105$

Neutron Studies of Polymer Dynamics in Intercalated Polymer-Clay

Nanocomposites

R. D. Ivkov115, P. M.Gehring ${ }^{105}$, N. Maliszewskyj $\left.\right|^{105}$, P. Papanek210, 105, and R. Krishnamoorti'194

Order-Disorder Transition in Highly Asymmetric Block Copolymers T. Lodge203, K. Hanley ${ }^{203}$, and X. Wang 203

Phase Behavior of Confined Polymer Films

S. K. Kumar ${ }^{137}$, R. Jones ${ }^{137}$, L.-T. Ho ${ }^{199}, 105$, and R. Briber 199

Phase Behavior of Multi-Component Polymer Systems in

Compressible Solvents

J. Watkins ${ }^{201}$, G. Brown ${ }^{201}$, V. RamachandraRa0201, B. Vogt201, and T. P. Russell201

Phase Behavior of Pentablock Copolymers

F. Bates ${ }^{203}$, T. Lodge ${ }^{203}$, X. Wangen ${ }^{203}$, Y. Chen ${ }^{203}$, and C. Hardy ${ }^{203}$

Phase Separation in Polyolefin Blends

M. Modi194 and R. Krishnamoorti194

Phase Transitions in Multicomponent Polymer Blends

N. Balsara ${ }^{184}$ and J. H. Lee' ${ }^{16}$

Polyethylene/Polymethylene Blends: Wetting and Phase Transitions Y. Akpalu'15 and A. Karim ${ }^{115}$

Polymer Brushes Under Shear

R. D. Ivkov'105, P. D. Butler ${ }^{105,127}$, and S. K. Satija ${ }^{105}$

Polymer Interdiffusion Near the Polymer/Solid Interface

E. K. Lin ${ }^{115}$, D. J. Pochan190, W. L. Wu'15, S. K. Satija ${ }^{105}$, and R. Kolb37

Polymer Liquid-Crystal Ordering at an Interface

G. Lynn 218, E. K. Lin ${ }^{115}$, W.-L. Wu'115, and M. Dadmun ${ }^{218}$

Polymer Supercritical Fluid Solutions

M. McHugh ${ }^{227}$, T. diNoia ${ }^{71}$, J. vanZanten ${ }^{71}$, C. Kirby ${ }^{71}$, T. Kermis ${ }^{71}$, and S. Conroy 71

Polyolefin Polymer Blend Tie Chain Structures

D. Lohse ${ }^{37}$, R. Garner ${ }^{37}$, and M. Xenidou ${ }^{37}$

Pressure Dependence of Phase Behavior in Styrene/ $\mathrm{N}$-alkyl

Methacrylate Block Copolymers

A.-V. Ruzette ${ }^{83}$, P. Banerjee ${ }^{33}$, and A. Mayes ${ }^{83}$

Pressure Effects on the Aggregation of lonomers

D. Perahia25, T. Hill25 and X. Jiao 25

SANS Investigations on a New Class of Nylon-6 Polymers

M. Kreitschmann ${ }^{44}$, W. Pyckhout-Hintzen ${ }^{44}$, and D. Richter ${ }^{44}$

SANS Study of lonomer Blends

R. Tucker ${ }^{189}$, R. Weiss ${ }^{189}$, and C. C. Han ${ }^{115}$

SANS Study of Phase Separation in Model Polymer Networks B. Viers ${ }^{187}$

Shear Induced Polymer Desorption

E. K. Lin ${ }^{115}$, R. Kolb ${ }^{115}$, and W.-L. Wu ${ }^{115}$

Shear-Induced Polymer Melt Desorption From an Attractive Solid

Surface

E. K. Lin ${ }^{115}$, D. J. Pochan ${ }^{190}$, W.-L. Wu' ${ }^{115}$, S. K. Satija ${ }^{105}$, and R. Kolb ${ }^{37}$

Small Angle Neutron Scattering of Water Dispersable Arborescent

Graft Copolymers

S. Yun ${ }^{19}$, R. M. Briber ${ }^{199}$, M. Gauthier ${ }^{25}$, and B. J. Bauer ${ }^{115}$

Solution Properties of Dendrimer-Like Star Polymers A. Gast ${ }^{156}$ and J. Pople ${ }^{156}$

Solvent Vapor Induced Swelling of a Polymer Brush S. K. Satija105, R. D. Ivkov'105, P. D. Gallagher'105, and J. A. Dura ${ }^{105}$

Spinodal Decomposition in PS/PI Blends

H. Jinnai76, M. Hayashi76, and C. C. Han ${ }^{115}$

Strain-Induced Interfacial Fracture Between Epoxy and Silicon 0xide M. Kent 152 and W. F. McNamara ${ }^{152}$

Strained Polymer Networks

N. Gilra ${ }^{28}$, C. Cohen ${ }^{28,}$, R. M. Briber ${ }^{199}$, and T. Panagiotopolis 199

The Structure of Associative Polymers at Rest and Under Shear X. Meng ${ }^{140}$, I. Goldmints ${ }^{140}$, and W. Russel ${ }^{140}$ 
Structure of Filled Elastomer Thin Films

Y. Zuang ${ }^{157}$, B. Tan ${ }^{157}$, M. Rafailovich 157, J. Sokolov'157, and M. Y. Lin ${ }^{37,}, 105$

Structure of Fluorosilicone Networks

F. Horkay ${ }^{46}$ and E. Geissler 192

Structure of Nafion in Various Solvents

S. F. Trevino", 105, S. Young ${ }^{6}$, and N. Beck-Tan ${ }^{6}$

Surface Segregation in Binary Blends of Topologically Distinct

Polymers

M. D. Foster ${ }^{176}$, C. C. Greenberg ${ }^{176}$, and D. M. Teale ${ }^{176}$

Structure and Aggregation Kinetics of Asphaltenes

T. Mason ${ }^{37}$, M. Y. Lin ${ }^{37}, 105$, and E. Sirota ${ }^{37}$

Structure of Adsorbed Polyelectrolyte Multilayer Thin Films

C. Barrett ${ }^{33}$ and A. M. Mayes ${ }^{23}$

Structure and Morphology of Semicrystalline Polymer Thin Films D. J. Pochan ${ }^{190}$, E. K. Lin ${ }^{115}$, W.-L. Wu'115, S. K. Satija'105, C. F. Majkrzak' ${ }^{105}$, and R. Kolb ${ }^{37}$

Study of Thermodynamics in Star and Linear Polymers M. Foster ${ }^{176}$ and T. Zook ${ }^{176}$

Thermal Expansion and Glass Transition Behavior of Polymer Films:

Effect of the Free Surface and Confinement Between Non-Interacting

Surfaces

D. J. Pochan ${ }^{190}$, E. K. Lin ${ }^{115}$, W.-L. Wu115, S. K. Satija105, and S. Z. D. Cheng ${ }^{176}$

Thermal Expansion and Glass Transition of Thin Polymer Films

Sandwiched Between Polymer Layers

D. J. Pochan ${ }^{190}$, E. K. Lin ${ }^{115}$, and W.-L. Wu'15

\section{COMPLEX FLUIDS}

Effects of Alcohols and Salts on the Structure of Oil-in-Water

Microemulsions

J. Lettow33, J. Yinge3, and C. J. Glinka105

Effect of Shear Combined with a Proximate Surface on Surfactant

Sponge Phases and the Sponge to Lamellar Transition

W. A. Hamilton 127, P. D. Butler105, 127, G. Warr'217, and L. Porcar ${ }^{27}$

Electrostatic and Counterion-Specific Effects on Micelle Morphology

Probed with SANS

L. J. Magid218, P. D. Butler105, 127, Z. Han ${ }^{218}$, and X. Xu218

Field Driven Phase Transitions in Monolayers and Bilayers of

Amphiphiles at Electrode Surfaces

J. Lipkowski ${ }^{193}$, J. Majewskipo, and G. Smith80

Interactions of Charged Polymerized Rod-Like Micelles With

Oppositely Charged Surfaces

S. M. Choi199, 105 and S. R. Kline 105

Influence of Counterion Valence on Domain Size of Polyelectrolyte

Solution

Y. Zhang ${ }^{115}$ and E. Amis ${ }^{115}$

Magnetic Alignment of Discotic Liquid Crystals

B. Pate ${ }^{58}$ and S. M. Choi'199, 105

Microemulsion Structure at an Interface

G. Warr ${ }^{217}$, J. Schulz217, P. D. Butler105.127, and W. A. Hamilton ${ }^{127}$

Microstructure of Alkylpolyglucoside Micelles and Microemulsions L. D. Ryan ${ }^{190}$, D. lanspetro ${ }^{100}$, C. McKelvey ${ }^{190}$, J. Silas' ${ }^{190}$, and E. W. Kaler ${ }^{190}$

Octane Wetting of an Air/Water Interface

S. Satija ${ }^{105}$ and R. D. Ivkov'105

Phase Transitions in Ferrofluids

W.-L. Lou41, M. Y. Lin ${ }^{37}, 105$, D. C. Dender ${ }^{105}$, and J. W. Lynn ${ }^{105}$

Poiseuille-Flow-Induced Changes in the Structure of Surfactant

Micelles

L. Walker ${ }^{19}$, B. Poore ${ }^{19}$, M. Truong ${ }^{19}$, and Y. Christanti19

Polymer, Block Copolymer and Clay Solutions Under Shear G. Schmidt115, C. C. Han ${ }^{115}$, and P. D. Butler ${ }^{105,127}$

Polymerization of Rod-Like Micelles S. Svenson ${ }^{140}$, R. Prud'homme ${ }^{140}$, and P. Peiffer ${ }^{37}$
Pressure-Induced Swelling of Polymer Micelles in Supercritical Fluids M. McHugh227, T. diNoia ${ }^{71}$, J. vanZanten ${ }^{71}$, C. Kirby ${ }^{71}$, T. Kermis ${ }^{71}$, and S. Conroy ${ }^{7}$

SANS Determination of the Persistence Length in Rod-Like Micelles L. J. Magid218, P. D. Butler 105, 127, and Z. Li218

SANS Determination of the Structure of Vitrified Microemulsions G. Warr216, P.R. Harrowell217, A. Widmer-Cooper217, and P. D. Butler ${ }^{105}, 127$

SANS From Nanodroplet Aerosols

B. Wyslouzi|231, C. Heath231, K Streletzky231, G. Wilemski205, and R. Strey ${ }^{188}$

SANS and Reflectivity Studies of Complex Polymer Mixtures

R. Briber ${ }^{199}$, B. J. Bauer15, and M. Gauthier225

Solution Structure of Novel Polymerizable Surfactants S. R. Kline ${ }^{105}$

Structure and Aggregation Behavior of Aqueous Solutions of Fulvic Acid

M. Diallo ${ }^{18}$ and C. J. Glinka ${ }^{105}$

Structure and Aggregation of Soot in Flowing Motor Oil M. Y. Lin ${ }^{37}$, M. Francisco ${ }^{37}$, and E. Sirota ${ }^{37}$

Structure of Dendritic Diblock Copolymer Langmuir Films P. Hammond ${ }^{83}$ and J. lyere ${ }^{83}$

Structure of Wax in Solution

E. Sirota ${ }^{37}$ and M. Y. Lin ${ }^{37,105}$

Vesicle and Micelle Formation of Block Polypeptides

D. J. Pochan ${ }^{190}$ and T. Deming ${ }^{184}$

\section{CONDENSED MATTER PHYSICS}

Anomaly in the Magnetic Phase Diagram of $\mathrm{U}_{1-x} \mathrm{Y}_{x} \mathrm{Pd}_{2} \mathrm{Al}_{3}$

T. M. Kelley'181, W. P. Beyermann ${ }^{181}$, R. A. Robinson ${ }^{80}$, J. W. Lynn ${ }^{105}$, E. J.

Freeman ${ }^{182}$, E. D. Bauer ${ }^{182}$, and M. B. Maple ${ }^{182}$

Antiferromagnetic Ordering of Composite Spin Degrees of Freedom in $\mathrm{La}_{4} \mathrm{Cu}_{3} \mathrm{MoO}_{12}$

M. Azuma ${ }^{76}$, C. Broholm 71 105, and Y. Qiu ${ }^{71}$

Antiferromagnetic Structure Determination: Exchange-Based MnPd-

Based Thin Films

J. A. Borchers ${ }^{105}$, R. F. Farrow ${ }^{55}$, and M. Toney ${ }^{55}$

Antiferromagnetic Structure Determination in NiO Nanoparticles

R. Kodama ${ }^{101}$ and J. A. Borchers ${ }^{105}$

Asymmetric Magnetization Reversal in Positive Exchange Bias

Hysteresis Loops

M. Fitzsimmons ${ }^{80}$, A. Hoffman ${ }^{80}$, I. K. Schuler ${ }^{182}$, and C. Leighton ${ }^{182}$

Charge, Orbital Magnetic Ordering and Two-Phase Coexistence in

$\mathrm{La}_{1 / 2} \mathrm{Ca}_{1 / 2} \mathrm{MnO}_{3}$

Q. Z. Huang' 199,105 , J. W. Lynn 105, V. Smolyaninova'199, K. Ghosh'199, R. L.

Greene ${ }^{199}$, D. C. Dender 105 , R. W. Erwin ${ }^{105}$, and A. Santor ${ }^{105}$

Critical Exponent in Equilibrium $\mathrm{Fe}_{.81} \mathrm{Zn}_{.19} \mathrm{~F}_{2}$ Thin Film Random Field

System

D. Belanger ${ }^{185}$, R. Erwin ${ }^{105}$, D. Lederman ${ }^{230}$

Crystal and Magnetic Structures of FeOCI

W. H. Li94, S. Y. Wu ${ }^{94}$, C. G. Wu ${ }^{94}$, and J. W. Lynn ${ }^{105}$

Crystal and Magnetic Structures of $\mathrm{La}_{3} \mathrm{RuO}_{7}, \mathrm{La}_{7} \mathrm{Ru}_{8} \mathrm{O}_{26}$ and $\mathrm{La}_{5} \mathrm{Ru}_{2} \mathrm{O}_{12}$ Q. Z. Huang ${ }^{199},{ }^{105}$, R. Cava ${ }^{140}$, R. W. Erwin' ${ }^{105}$, and J. W. Lynn ${ }^{105}$

Crystal and Magnetic Structures of $\mathrm{PrBa}_{2} \mathrm{Cu}_{4} \mathrm{O}_{8}$ W.H. Li94, S. Y. Wu ${ }^{94}$, and J. W Lynn ${ }^{105}$

Crystal and Magnetic Structures of $\mathrm{Sr}_{3} \mathrm{Ru}_{2} \mathrm{O}_{7}$ and $\mathrm{Sr}_{4} \mathrm{Ru}_{3} \mathrm{O}_{10}$ R. L. Harlow ${ }^{33}$, M. K. Crawford ${ }^{33}$, J. W. Lynn ${ }^{105}$, Q. Z. Huang ${ }^{199,} 105, \mathrm{~S}$. Skanthakumar ${ }^{4}$, and G. Ca041

Crystal and Magnetic Structures of $\mathrm{Sr}_{4} \mathrm{Fe}_{2} \mathrm{CO}_{9}$

Q. Z. Huang ${ }^{199}, 105$, R. Cava ${ }^{140}$, R. W. Erwin ${ }^{105}$, and J. W. Lynn ${ }^{105}$

Crystal and Magnetic Structures of $\mathrm{TbBaCO}_{2} \mathrm{O}_{6}$

Q. Z. Huang 199, 105, I. Troyanchuk'2, and J. W. Lynn'105

Crystal and Magnetic Structures of $\mathrm{ZnCr}_{1.4} \mathrm{Ga}_{0.6} \mathrm{O}_{4}$

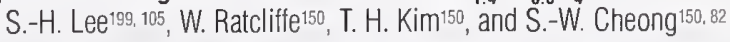

Crystal Structure and Magnetic Ordering in $\mathrm{CoMoO}_{3}$

Q. Z. Huang ${ }^{199} 105$ J. W. Lynn 105, R. W. Erwin ${ }^{105}$, and M. Crawford ${ }^{33}$ 
Crystal Structure and Magnetic Ordering in the Perovskite

$\mathrm{SrRu}_{0.33} \mathrm{Mn}_{0.67} \mathrm{O}_{3}$

Q. Z. Huang ${ }^{199,} 105$ and R. J. Cava ${ }^{140}$

Crystal Structure and Magnetic Properties of $\mathrm{Tb}_{3}\left(\mathrm{Fe}_{1-\mathrm{x}} \mathrm{Co}_{x}\right)_{27.5} \mathrm{Ti}_{1.5}$ Alloy V. G. Harris ${ }^{101}$ and Q. Z. Huang ${ }^{199,} 105$

Crystallographic and Magnetic Structure of a Series of Lanthanide

Copper Oxides: $\mathrm{LnCu}_{2} \mathrm{O}_{4}$

B. A. Reisner ${ }^{105,157}$, A. Stacy ${ }^{178}$, and J. Luce ${ }^{178}$

Crystallographic and Magnetic Structure of $\mathrm{Tb}_{2} \mathrm{Ni}_{3} \mathrm{Si}_{5}$

F. Bourdarot27, 105, J. W. Lynn105, and L. Guptat ${ }^{160}$

Crystallographic Study of $\mathrm{La}_{0.67} \mathrm{Mg}_{.33} \mathrm{MnO}_{3}$

C. P. Adams ${ }^{199}, 105$, J. W. Lynn ${ }^{105}$, R. G. Williams ${ }^{198}$, and G. M. Zha0 ${ }^{199}$

Density of States in Isotope Substituted $\mathrm{La}_{5 / 8} \mathrm{Ca}_{3 / 8} \mathrm{MnO}_{3}$

C. A. Adams ${ }^{199,}{ }^{105}$, J. W. Lynn ${ }^{105}$, S-W. Cheong 150,82, W. Ratcliffe ${ }^{150}$, and

L. Young 150

Determination of the Dimensionality of the Gapped Spin-1/2 System

in $\mathrm{CuHpCl}$

M. Stone ${ }^{71}$, D. H. Reich 71 , and C. Broholm ${ }^{71,}, 105$

Diffuse Scattering From Nano-Polar Regions in Relaxor Ferroelectric

$\mathrm{K}_{1-\mathrm{x}} \mathrm{Li}_{\mathrm{x}} \mathrm{TaO}_{3}$

G. Yong ${ }^{78}$, R. W. Erwin ${ }^{105}$, and J. Toulouse ${ }^{78}$

Dynamics of the Relaxor PMN

P. M. Gehring ${ }^{105}$, G. Shirane ${ }^{15}$, and S. B. Vakhrushev 69

Effect of Superconductivity on the Lattice Dynamics of $\mathrm{Ba}_{0.6} \mathrm{~K}_{0.4} \mathrm{BiO}_{3}$

Y. S. Lee ${ }^{105}$, C. P. Adams ${ }^{199}$, 105, J. W. Lynn ${ }^{105}$, and S. N. Barilo ${ }^{12}$

Effects of $\mathrm{H}$ on Magnetic Coupling in FeV Superlattices

B. Horvarsson ${ }^{146}$, G. Andersson ${ }^{146}$, J. A. Dura ${ }^{105}$, T. Udovic ${ }^{105}$, and C. F. Majkrzak ${ }^{105}$

Field Dependence of Magnetic Ordering in a System of Weakly

Coupled Antiferromagnetic Spin-1/2

Y. Chen ${ }^{71}$, D. H. Reich ${ }^{71}$, and C. Broholm ${ }^{71,} 105$

Field Induced Magnetic Order in the Antiferromagnetic Spin-1 Chain NDMAP

Y. Chen ${ }^{71}$, Z. Honda ${ }^{62}$, A. Zheludevi5, C. Broholm ${ }^{71}$ 105, K. Katsumata ${ }^{62}$, and S.

M. Shapiro ${ }^{15}$

Field Induced Magnetic Order in Spin Ice $\mathrm{Dy}_{2} \mathrm{Ti}_{2} \mathrm{O}_{7}$

Y. Qiü11, I. Hagemann ${ }^{140}$, R. J. Cava ${ }^{140}$, and C. Broholm¹, 105

Field Induced Ordering in Spin Ice $\mathrm{Dy}_{2} \mathrm{Ti}_{2} \mathrm{O}_{7}$

I. Hagemann ${ }^{140}$, R. J. Cava ${ }^{140}$, C. Broholmm ${ }^{71}, 105$, and Y. Qiu ${ }^{71}$

Field and Temperature Dependent Neutron Scattering Study of $\mathrm{Mn}_{12}$

Magnetic Molecules

T. Yildiriming and S.-H. Lee ${ }^{199,105}$

First-Principles Investigation of Structural and Electronic Properties of

Solid Cubane and Its Derivatives

T. Yildirim ${ }^{105}$, S. Ciraci ${ }^{14}$, and C. Kilic ${ }^{14}$

Flux Lattice Structure in $\mathrm{Nb}$ and $\mathrm{BaKBiO}_{3}$

X. Ling ${ }^{17}$, D. C. Dender ${ }^{105,}{ }^{199}$, J. W. Lynn ${ }^{105}$, S. M. Choi ${ }^{199,}{ }^{105}$, and S. Park ${ }^{17}$

Gap in the Excitation Spectrum of the Frustrated Two Dimensional

Spin-1/2 Magnet PHCC

M. Stone ${ }^{71}$, D. H. Reich ${ }^{71}$, C. Broholm ${ }^{71}, 105$, and I. Zaliznyak ${ }^{15}$

Ground-State Selection in FCC and BCT Antiferromagnets Due to

Quantum Disorder

T. Yildirim ${ }^{105}$, A. B. Harris ${ }^{210}$, and E. F. Shender ${ }^{178}$

Incommensurate and Commensurate Magnetic Structures in the Magnetoresistive $\mathrm{R}_{2} \mathrm{Ni}_{3} \mathrm{Si}_{5}$ Materials

F. Bourdarot ${ }^{27}, 105$, S. Skanthakumar ${ }^{4}$, J. W. Lynn ${ }^{105}$, and L. C. Gupta ${ }^{160}$

Incommensurate Magnetic Structure of CeRhIn

W. Bao $0^{80}$, P. G. Pagliuso ${ }^{105}$, J. L. Sarra0 ${ }^{80}$, J. D. Thompson ${ }^{105}$, Z. Fish ${ }^{105}$, J. W.

Lynn ${ }^{105}$, and R. W. Erwin ${ }^{105}$

Incommensurate Spin Fluctuations and Structural Transitions in

Excess Oxygen-Doped $\mathrm{La}_{2} \mathrm{CuO}_{4+\mathrm{y}}$

Y. S. Lee ${ }^{105}$, R. J. Birgeneau83, B. Khaykovich ${ }^{83}$, M. A. Kastner ${ }^{83}$, and R. W.

Erwin 105

Influence of Interface Structure on the Magnetic Polarizability of a

$\mathrm{Pd}-\mathrm{Co}$ Alloy Overlayer in Close Proximity to $\mathrm{CoPt}_{3}$

M. Fitzsimmons ${ }^{80}$, F. Hellman'182, B. Maran ${ }^{182}$, and A. Shapiro ${ }^{182}$
Interfacial Magnetic Structure in Spin-Dependent Tunnel Junctions

K. V. O'Donovan ${ }^{105}$, J. A. Borchers ${ }^{105}$, and S. S. Parkin ${ }^{55}$

Interlayer Spin Coupling in FeMn-Based Spin Valves

J. A. Borchers ${ }^{105}$, C. F. Majkrzak ${ }^{105}$, A. Reilly ${ }^{26}$, W. P. Pratt, Jr. ${ }^{90}$, J. Bass ${ }^{90}$ and D. C. Dender ${ }^{105}$

Long-Range Magnetic Order in Hybrid Co/Cu Multilayers

J. A. Borchers ${ }^{105}$, W. Pratt ${ }^{90}$, and J. Bass 90

Local Spin Resonance in a Frustrated Antiferromagnetic $\mathrm{ZnCr}_{2} \mathrm{O}_{4}$ S.-H. Lee ${ }^{199,105}$, C. Broholm ${ }^{71.105}$, T. H. Kim ${ }^{150}$, W. Ratcliff150, and S-W. Cheong ${ }^{150,82}$

Low Energy Magnetic Correlations in the Quantum Critical Spin

System $\mathrm{CeNi}_{2} \mathrm{Ge}_{2}$

A. Schroeder ${ }^{73}$, D. McMorrow ${ }^{144}$, N. Beck-Christensen ${ }^{144}$, C. Broholm ${ }^{71}, 105$, Y.

Qiư? and G. Aepplitio3

Magnetic Correlations in Discontinuous $\mathrm{Co}_{0} \mathrm{SiO}_{2}$ Thin Films and

Multilayers

S. Sankar ${ }^{199}$, A. E. Berkowitz ${ }^{182}$, D. C. Dender ${ }^{105,}{ }^{199}$, J. A. Borchers ${ }^{105}$, R. W.

Erwin $^{105}$, S. R. Kline ${ }^{105}$, J. A. Dura ${ }^{105}$, and C. F. Majkrzak ${ }^{105}$

Magnetic Correlations and Lattice Distortions in the Bilayer Manganite

$\mathrm{La}_{1.2} \mathrm{Sr}_{1.8} \mathrm{Mn}_{2} \mathrm{O}_{7}$

L. Vasiliu-Doloc'123, S. Rosenbranz', R. Osborn" ${ }^{4}$ S. K. Sinha4 , J. W. Lynn ${ }^{105}$, and J. F. Mitchell ${ }^{4}$

Magnetic Correlations in Li-Doped $\mathrm{La}_{2} \mathrm{CuO}_{4}$

W. Bao ${ }^{81}$ and S.-H. Lee $e^{199,105}$

Magnetic Correlations in a Geometrically Frustrated Magnet $2 n V_{2} \mathrm{O}_{4}$ S. H. Lee ${ }^{199}, 105$, C. Broholm77, 105, and Y. Ueda'169

Magnetic Correlations in the Heavy Fermion System $\mathrm{LiV}_{2} \mathrm{O}_{4}$ S.-H. Lee ${ }^{199,105}$, C. Broholm $1 m^{71}$, 105, and Y. Ueda ${ }^{169}$

Magnetic Correlations in a System of Coupled Spin-1/2 Dimers

CuHPCI

M. Stone ${ }^{71}$, Y. Chen ${ }^{71}$, D. Reich ${ }^{71}$, and C. Broholm ${ }^{71}, 105$

Magnetic and Crystal Structure Phase Transitions in $R_{1-x} \mathrm{Ba}_{x} \mathrm{CoO}_{3-y}(\mathrm{R}$ $=\mathrm{Nd}, \mathrm{Gd}$ )

I. O. Troyanchuk'12, D. D. Khalyavin'12, T. K. Solovykh'12, H. Szymczak'2, Q. Z. Huang ${ }^{199}, 105$, and J. W. Lynn ${ }^{105}$

Magnetic and Crystal Structures of $\mathrm{CaRuO}_{3}$ J. W. Lynn ${ }^{105}$ and I. Felner ${ }^{143}$

Magnetic and Crystal Structures of the New Heavy Fermion Compound CeRhSn

E. Granado ${ }^{182}$, J. W. Lynn ${ }^{105}$, E. D. Bauer ${ }^{182}$, A. Zapf182, and M. B. Maple ${ }^{182}$

Magnetic Domain Structure of Thermally Treated Nanocrystalline

$\mathrm{Ni}_{3} \mathrm{Fe}$

H. Frase ${ }^{18}$ and L. Robertson ${ }^{127}$

Magnetic Excitations in Alternating 1D Magnetic Chains

A. B. Harris 210 and T. Yildirim 105

Magnetic Excitations in Mn-12 and Fe-8 Magnetic Clusters

T. Yildirim ${ }^{105}$ and S. H. Lee ${ }^{199,105}$

Magnetic Order and Crystal Field Effects in RNiBC

F. Bourdarot27, 105, J. W. Lynn 105, Q. Z. Huang 199, 105, D. R. Sanchez22, M. B.

Fontes22, J. C. Trochez22, and E. Baggio-Saitovitch22

Magnetic Order in EuMn $\mathbf{n}_{2} \mathrm{P}$

J. Y. Chan ${ }^{109}$, J. W. Lynn ${ }^{105}$, S. M. Kauzlarich'179, A. Payne ${ }^{179}$, and B. A. Reisner 105

Magnetic Order in the Ferromagnetic Superconductors $\mathrm{RuSr}_{2}{ }^{160} \mathrm{GdCu}_{2} \mathrm{O}_{8}$ J. W. Lynn ${ }^{105}$ B. Keimer ${ }^{84,140}$, C. Ulich ${ }^{140}$, C. Bernhard ${ }^{140}$, and J. L. Tallon ${ }^{58}$

Magnetic Order in Pr-Containing Cuprate Superconductors W. H. Lipa, K. C. Lee 94, H. C. Ku'100, and J. W. Lynn 105

Magnetic Order, Structure and Spin Dynamics of $\left(\mathrm{La}_{1-\mathrm{x}} \mathrm{Ca}_{\mathrm{x}}\right) \mathrm{MnO}_{3}$ J. W. Lynn ${ }^{105}$, R. W. Erwin ${ }^{105}$, J. A. Borchers ${ }^{105}$, Q. Z. Huang ${ }^{199}$, ${ }^{105}$, A. Santoro ${ }^{105}$, K. Ghosh ${ }^{199}$, and R. L. Greene ${ }^{199}$

Magnetic Order in the Superconductor $\mathrm{RNi}_{2} \mathrm{~B}_{2} \mathrm{C}$ J. W. Lynn ${ }^{105}$, S. Skanthakumar', Z. Hossain ${ }^{160}$, L. C. Gupta160, R. Nagarajan160, and C. Godart?

Magnetic Ordering in $\mathrm{CO}_{2} \mathrm{SnO}_{4}$

M. K. Crawford 33 , R. B. Flippen ${ }^{33}$, R. L. Harlow33, J. Hormadaly33, J. W. Lynn ${ }^{105}$, and Q. Z. Huang 199,105 
Magnetic Ordering of Exchange-Biased $\mathrm{Fe}_{3} \mathrm{O}_{4} / \mathrm{NiO}$ Superlattices J. A. Borchers ${ }^{105}$, R. W. Erwin ${ }^{105}$, Y. ljiri128, D. M. Y. Lind ${ }^{41}$, P. G. Ivanov ${ }^{41}$ A Qasba ${ }^{105}$, K. V. O'Donovan ${ }^{105}$

Magnetic Ordering in the Frustrated Heavy Fermion System UNi ${ }_{4} B$ J. Mydosh ${ }^{78}$, C. Broholm ${ }^{71}$, 105, and Y. Qiu ${ }^{71}$

Magnetic Ordering of $\mathrm{Mn}$ in $\mathrm{Nd}_{1-x} \mathrm{Ca}_{\mathbf{x}} \mathrm{MnO}_{3}$ S. Y. Wu94, W-H. Li94, K. C. Lee ${ }^{94}$, J. W. Lynn ${ }^{105}$, R. S. Liu ${ }^{99}$, J. B. Wu ${ }^{99}$, and C. Y. Huang ${ }^{105,} 199$

Magnetic Ordering and Structure of $\mathrm{PrBa}_{2} \mathrm{Fe}_{3} \mathrm{O}_{8}$ N. Rosov'105, J. W. Lynn ${ }^{105}$, M. Seyedahmadian'22, and T. Yeun ${ }^{82}$

Magnetic Properties of $\mathrm{HoVO}_{4}$ C. K. Loong ${ }^{4}$, S. Skanthakumar ${ }^{4}$, J. W. Lynn ${ }^{105}$, and G. K. Liu' ${ }^{199}$

Magnetic Proximity Effect in Pd A. Hotfmann 80 and M. Fitzsimmons ${ }^{80}$

Magnetic Structure of Charge Ordered $\mathrm{CaFeO}_{3}$ E. Moshopoulou15, D. E. Cox ${ }^{15}$, and P. Woodward ${ }^{129}$

Magnetic Structure and Interlayer Correlations in Ferromagnetic Superlattices EuS/Pbs H. Kepa ${ }^{223}$ and T. T. Giebultowicz ${ }^{132}$

The Magnetic Structure of [MnTPP][TCNE] 2PhMe by High-Resolution Neutron Powder Diffraction C. R. Kmety-Stevenson ${ }^{129}$, A. J. Epstein ${ }^{129}$, J. S. Miller ${ }^{220}$, E. J. Brandon ${ }^{220}$ and J. W. Lynn 105

Magnetism of Ferrihydrite Nanoparticles M. S. Seehra230, V. Suresh Babu230, and J. W. Lynn'105

Magnetization of Antiferromagnetically Ordered $\mathrm{UCu}_{1-x} \mathrm{Pd}_{x}$ T. M. Kelley 181 , W. P. Beyermannn ${ }^{181}$, R. A. Robinson ${ }^{80}$, J. W. Lynn ${ }^{105}$, E. J. Freeman ${ }^{182}$, E. D. Bauer ${ }^{182}$, R. Chau' ${ }^{182}$, and M. B. Maple ${ }^{182}$

Neutron Coherence Length and Non-Specular Scattering From Diffraction Gratings C. F. Majkrzak ${ }^{105}$ and N. F. Berk ${ }^{105}$

Neutron Scattering Study of the Nuclear and Magnetic Structure of RD, T. J. Udovic ${ }^{105}$, Q. Z. Huang ${ }^{199}{ }^{105}$, J. W. Lynn ${ }^{105}$, R. W. Erwin ${ }^{105}$, and J. J. Rushto5

Neutron Studies of New Incommensurate Magnetic Correlations Near the Lower Critical Concentration for Stripe Order in $\mathrm{La}_{1.6-x} \mathrm{Nd}_{0.4} \mathrm{Sr}_{x} \mathrm{CuO}$ J. M. Tranquada ${ }^{15}$, N. Ichikawa ${ }^{169}$, P. M. Gehring ${ }^{105}$, and S.-H. Lee ${ }^{199 .}{ }^{105}$

Observation of a Magnetic Spiral in an Exchange-Spring Magnet K. V. O'Donovan ${ }^{105}$, J. A. Borchers ${ }^{105}$, and E. E. Fullerton ${ }^{105}$

Oxygen Isotope Effect on the Spin and Lattice Dynamics of $\mathrm{La}_{5 / 8} \mathrm{Ca}_{3 / 8} \mathrm{MnO}_{3}$

C. P. Adams ${ }^{199}, 105$, Y. S. Lee ${ }^{105}$, J. W. Lynn ${ }^{105}$, V. Smolyaninova ${ }^{199}$, G. M. Zha0 ${ }^{199}$, R. L. Greene ${ }^{199}$, S.-W. Cheong 150,82 and W. Ratcliffe 150

Polarized Neutron Diffraction Studies of Magnetic Ordering in Exchange-Biased $\mathrm{Fe}_{3} \mathrm{O}_{4} / \mathrm{CoO}$ Superlattices

Y. ljirit28, J. A. Borchers ${ }^{105}$, R. W. Erwin'105, S.-H. Lee ${ }^{199,}{ }^{105}$, C. F. Majkrzak ${ }^{105}$, P. J. van der Zaag ${ }^{138}$, and R. M. Wolf 138

Polarized Neutron Reflectivity of an Array of Cobalt Dots S. Adenwalla ${ }^{207}$ and C. F. Majkrzak ${ }^{105}$

Polarized Neutron Scattering Measurements of the Oxygen Moment in (La,Sr) $\mathrm{MnO}_{3}$ P. M. Gehring ${ }^{105}$, G. Shirane ${ }^{15}$, H. Hirotat68, and J. W. Lynn ${ }^{105}$

Polarized Neutron Studies of Magnetic Interfacial Roughness in EuTe/ PbTe Superlattices H. Kepa23, T. Giebultowicz ${ }^{132}$, G. Bauer ${ }^{132}$, and C. F. Majkrzak ${ }^{105}$

Pr Magnetic Order and Spin Dynamics in the Cuprates J. W. Lynn ${ }^{105}$, N. Rosov105, S. N. Barilo ${ }^{12}$, L. Kurnevitch ${ }^{12}$, and A. Zhokhov'149

Pressure Dependence of the Magnetic Order in $\mathrm{UGe}_{2}$ J. W. Lynn ${ }^{105}$, E. J. Freeman ${ }^{182}$, and M. B. Maple ${ }^{182}$

Q-Dependence of the Spin Flip Scattering From Exchange Coupling Across Fe-FeF ${ }_{2}$ Interfaces Measured With Spin Polarized Neutron Diffraction With Polarization Analysis M. Fitzsimmons ${ }^{80}$ and I. K. Schuler ${ }^{182}$, C. L. Leighton ${ }^{182}$, and A. Hoffman ${ }^{80}$

Quantum Percolation on the Square Lattice: Crystal Growth, Neutron Scattering and Monte Carlo O. P. Vajk ${ }^{156}$, M. Greven ${ }^{156}$, P. K. Mang ${ }^{156}$, and J. W. Lynn ${ }^{105}$
Reflectivity Measurements on Spin Valve Structures

D. Sarkisov214 and M. Dabaghian ${ }^{214}$

Search for Spin Density Waves in $\mathrm{Pb}$

A. Overhauser ${ }^{141}$ and J. W. Lynn ${ }^{105}$

Self-Assembled Polymer-Like Structures and Effect of Fields in Phase-

Separated Magnetic Fluids

M. Y. Lin ${ }^{37,}$, 105, T. Du ${ }^{40}$, J. W. Lynn ${ }^{105}$, and W. Luo $0^{40}$

Single-Ion Anisotropy and Crystal Field Effects in $\mathrm{R}_{2} \mathrm{CuO}_{4}(\mathrm{R}=\mathrm{Nd}$,

Sm, Pr, ...)

T. Yildirim ${ }^{105}$, R. Sachidanandam ${ }^{165}$, A. Aharony ${ }^{165}$, and A. B. Harris ${ }^{210}$

Spin Correlations in ( $\mathrm{Nd}-\mathrm{Ce})_{2} \mathrm{CuO}_{4}$

P. K. Mang ${ }^{156}$, M. Greven ${ }^{156}$, and J. W. Lynn ${ }^{105}$

Spin Dynamics of $\mathrm{CrO}_{2}$

E. Granado ${ }^{199}, 105$, J. W. Lynn ${ }^{105}$, and M. Rubinstein ${ }^{10}$

Spin Dynamics of the $\mathrm{Mn}$ lons in $\mathrm{La}_{1-\mathrm{x}} \mathrm{Ba}_{\mathrm{x}} \mathrm{MnO}_{3}$ and $\mathrm{Pr}_{1-\mathrm{x}} \mathrm{Ba}_{\mathrm{x}} \mathrm{MnO}_{3}$

J. W. Lynn ${ }^{105}$, L. Vasiliu-Doloc ${ }^{123}$, K. Ghoshing, R. L. Greene ${ }^{199}$, and S. Barilo ${ }^{12}$

Spin Dynamics in LaTiO

B. Keimer ${ }^{84,140}$, A. Ivanov'59, J. W. Lynn ${ }^{105}$, Y. Taguchi ${ }^{169}$, and Y. Tokura ${ }^{169}$

Spin Dynamics and Change Ordering in Single Crystal $\mathrm{La}_{7} \mathrm{Ca}_{3} \mathrm{MnO}_{3}$ C. P. Adams ${ }^{199}, 105$, J. W. Lynn ${ }^{105}$, and Y. M. Mukovskii92

Spin Dynamics of Strongly Doped $\mathrm{La}_{1-\mathrm{x}} \mathrm{Sr}_{\mathrm{x}} \mathrm{MnO}_{3}$ L. Vasiliu-Doloc ${ }^{123}$, J. W. Lynn ${ }^{105}$, Y. M. Mukovskii ${ }^{92}$, A. A. Arsenov ${ }^{92}$, and D. A. Shulyatev ${ }^{2}$

Spin Order and Dynamics in $\mathrm{Bi}_{2} \mathrm{Sr}_{2} \mathrm{C}_{0} \mathrm{O}_{5+d}$ Y. S. Lee ${ }^{105}$, J. W. Lynn ${ }^{105}$, F. C. Chou ${ }^{83}$, M. A. Kastner ${ }^{83}$, T. Imai83, and J. K. Thomas ${ }^{83}$

Static and Dynamic Properties of Spin and Charge Ordering in $\mathrm{La}_{2}$ $\mathrm{Sr}_{x} \mathrm{NiO}$

S.-H. Lee ${ }^{199,}{ }^{105}$, J. M. Tranquada ${ }^{15}$, and K. Yamada ${ }^{76}$

Structural Phase Transition and Magnetic Order in Heavy Fermion Compounds $\mathrm{Ce}_{3} \mathrm{M}$ with $\mathrm{M}=\mathrm{Al}$, In and $\mathrm{Sn}$

W.-H. Li94, K. C. Lee ${ }^{94}$, Y. Y. Chen ${ }^{82}$, and J. W. Lynn ${ }^{105}$

Structure and Frustrated Magnetism in $\mathrm{KFe}_{3}(\mathrm{OD})_{6}\left(\mathrm{SO}_{4}\right)_{2}$ Y. S. Lee ${ }^{105,}{ }^{199}$, J. W. Lynn ${ }^{105}$, Q. Z. Huang ${ }^{199}, 105$, B. Toby'105, S.-H. Lee ${ }^{105,199}$, D. Grohol ${ }^{33}$, and D. Nocera ${ }^{83}$

Structure and Magnetic Order in the $\mathrm{A}_{2} \mathrm{BO}_{4}$ Spinel $\left(A=\left(\mathrm{Co}_{0}, \mathrm{Ru}\right), \mathrm{B}\right.$ $=(\mathrm{Zn}, \mathrm{Ga}, \mathrm{Ge}))$

Q. Z. Huang ${ }^{199} 1^{105}$, J. W. Lynn ${ }^{105}$, R. W. Erwin ${ }^{105}$, M. Crawford ${ }^{33}$, R. L. Harlow ${ }^{33}$ and E. M. McCarron ${ }^{33}$

Structure and Magnetic Order in $\mathrm{Sr}_{2} \mathrm{Y}_{0.8} \mathrm{Ca}_{0.2} \mathrm{Co}_{2} \mathrm{O}_{6}$ Q. Z. Huang ${ }^{199 .} 105$ J. W. Lynn ${ }^{105}$, R. W. Erwin ${ }^{105}$, and R. J. Cava ${ }^{140}$

Structure and Magnetic Ordering of $M "\left[N(C N)_{2}\right]_{2}\left(M=C_{0}, N i, M n\right)$ J. L. Manson ${ }^{4}$, C. R. Kmety-Stevenson ${ }^{129}$, Q. Z. Huang ${ }^{199}{ }^{105}$, J. W. Lynn ${ }^{105}$ G. Bendele ${ }^{157}$, S. Pagola157, P. W. Stephens ${ }^{157}$, L. M. Kiable-SandS ${ }^{15}$, A. L. Rheingold ${ }^{15}$, A. J. Epstein ${ }^{129}$, and J. S. Miller220

Structure and Magnetic Properties in the $\mathrm{Sr}_{1-x}\left(\mathrm{La}_{0.5} \mathrm{Na}_{0.5}\right)_{x} \mathrm{RuO} \mathrm{O}_{3}$ Solid Solution

Q. Z. Huang ${ }^{199 .} 105$ and R. J. Cava ${ }^{140}$

Structure of $\mathrm{YCO}_{0.5} \mathrm{Mn}_{0.5} \mathrm{O}_{3-\mathrm{x}}, \mathrm{Tb}_{0.5} \mathrm{Ba}_{0.5} \mathrm{CoO}_{x}$ and $\mathrm{Nd}_{0.5} \mathrm{Ba}_{0.5} \mathrm{CoO}_{x}$ Q. Z. Huang 199.105 , J. W. Lynn ${ }^{105}$ R. W. Erwin ${ }^{105}$, and I. Troyanchuk ${ }^{12}$

Structure of PZN-PT Under Electric Field

P. M. Gehring ${ }^{105}$, G. Shirane ${ }^{15}$, K. Hirotat ${ }^{168}$, S.-E. Park ${ }^{137}$, and T. R. Shnut ${ }^{137}$

Structures of $\mathrm{YM}_{2} \mathrm{Cu}_{2.85} \mathrm{Re}_{0.15} \mathrm{O}_{7+\mathrm{x}}(\mathrm{M}=\mathrm{Ba}$ and $\mathrm{Sr}) \mathrm{High} \mathrm{T}_{\mathrm{c}}$

Superconductors

Q. Z. Huang ${ }^{199}$, 105 , M. Marezio66, F. Licci66, and A. Santoro ${ }^{105}$

Study of Metallic Stripes and Charge Order in Superconducting

$\mathrm{La}_{1.6-x} \mathrm{Nd}_{0.4} \mathrm{Sr}_{x} \mathrm{CuO}_{4}$

N. Ichikawa ${ }^{169}$, S. Uchida $^{169}$, J. M. Tranquada ${ }^{15}$, T. Niemolier ${ }^{52}$, P. M. Gehring ${ }^{105}$ S.-H. Lee ${ }^{105,} 199$, and J. R.Schneider ${ }^{52}$

Superconductivity in $\mathrm{La}_{2-x} \mathrm{Sr}_{x} \mathrm{CuO}$ S. Wakimoto ${ }^{168}$, S. Ueki168, K. Hirota'168, K. Yamada'168, Y. Endoh'168, R. J. Birgeneau ${ }^{83}$, Y. S. Lee ${ }^{83}$, M. A. Kastner ${ }^{83}$, G. Shirane ${ }^{15}$, S.-H. Lee ${ }^{105.199}$, and P. M. Gehring 105 
Superconductivity, Magnetic Fluctuations and Magnetic Order in

$\mathrm{TbSr}_{2} \mathrm{Cu}_{2.69} \mathrm{Mo}_{0.31} \mathrm{O}_{7}$

W.-H. Li" ${ }^{94}$ W. Y. Chuang95 Y. Wu94, K. C. Lee ${ }^{94}$, J. W. Lynn'105, H. L. Tsay ${ }^{98}$ and H. D. Yang ${ }^{98}$

Surface Spin Ordering in $\mathrm{LaFeO}_{3}$ Thin Films J. A. Borchers ${ }^{105}$, J. Stohr ${ }^{55}$, and M. Toney5

Synthesis, Crystal Structure and Magnetic Order of the Ion

Oxycarbonate $\mathrm{Sr}_{4} \mathrm{Fe}_{2} \mathrm{CO}_{6}$

K. Yamaura'140, Q. Z. Huang ${ }^{199}, 105$, J. W. Lynn ${ }^{105}$, R. W. Erwin ${ }^{105}$, and R. J. Cava ${ }^{140}$

Temperature Evolution of the Spin Excitation Spectrum in a Reentrant

Spin Glass $\mathrm{Fe}_{0.7} \mathrm{Al}_{0.3}$ W. Ba0 ${ }^{80}$, S. Shapiro ${ }^{15}$, and S.-H. Lee ${ }^{199,105}$

Two-Step Short Range Magnetic Order in the Spinel LiMn, $\mathrm{O}_{4}$

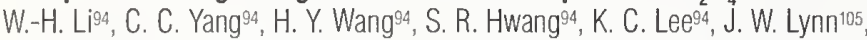
R. S. Liu ${ }^{94}$, and C. H. Chen ${ }^{94}$

Variation in the Structural and Magnetic Properties Induced by La

Doping in $\operatorname{Pr}\left(\mathrm{Ba}_{1-\mathrm{x}} \mathrm{La}_{x}\right) \mathrm{Cu}_{3} \mathrm{O}_{7}$

H. Y. Wang ${ }^{94}$, C. H. Chang ${ }^{94}$, S. R. Hwang ${ }^{94}$, W.-H. Li ${ }^{94}$, K. C. Lee ${ }^{94}$, J. W.

Lynn ${ }^{105}$, H. M. Luo $0^{94}$, and H. C. Ku'100

Vortex Lattice and Magnetic Order in ErNi, BrC

P. Gammel ${ }^{193}$, S. Lopez ${ }^{193}$, D. C. Denderio5, S. M. Choi ${ }^{199,}{ }^{105}$, and J. W. Lynn'105

Vortex Lattice Structure in High-T Bi-2212

B. Keimer ${ }^{140,84}$, T. Fong ${ }^{140}$, and J. W. Lynn ${ }^{105}$

$X$-ray Dichroism/Neutron Reflectivity Analysis of In-Plane

Ferromagnetic Domains in $\mathrm{Co} / \mathrm{Cu}$

Y. Idzerdaa101, J. Bass ${ }^{90}$, W. Pratt90, J. A. Borchers ${ }^{105}$, and K. V. O'Donovan105

\section{BIOMOLECULAR SCIENCE}

Amplitudes of Protein Motion: Resolution of a Discrepancy Between Neutron Scattering Experiments and MD Simulations

M. Tarek200, 105 , G. L. Martyna ${ }^{58}$, and D. J. Tobias ${ }^{180}$

Characterization of Polymeric Membrane Mimetic Films Using Neutron Reflectivity

S. Krueger ${ }^{105}$, C. F. Majkrzak ${ }^{105}$, C. W. Meuse ${ }^{107}$, and E. Chaikof ${ }^{36}$

Cryopreservation of Proteins

A. M. Tsai105, C. L. Soles ${ }^{115}$ and J. Shiloach 96

Determination of the Location of the Lipid Anchor in Outer Surface

Protein A of Borrelia Burgdorfei by SANS

D. Schneider ${ }^{15}$, C. Lawson ${ }^{15}$, and V. Graziano ${ }^{15}$

The Dynamics of Fibrinogen and Related Fibrin Gels

R. Nossal96, A. M. Tsai105, and D. Sackett96

The Dynamics of Protein Hydration Water: A Quantitative Comparison of MD Simulations and Neutron Scattering Experiments

M. Tarek200, 105 and D. J. Tobias ${ }^{180}$

Environmental Dependence of the Dynamics of Protein Hydration Water

M. Tarek ${ }^{200,105}$ and D. J. Tobias 180

Gelation of Methylcellulose

C.-I. Huang ${ }^{203}$ and T. Lodge 203

Incoherent Scattering From Lipid Bilayers and Bilayers With Channel Proteins Imbedded

S.-H. Chen" ${ }^{83}$, C. Lia083, T. Weiss ${ }^{145}$ and C. M. Brown'199, 105

Interaction of the Peptide Penetration with Phospholipid Double Bilayers

G. Fragneto ${ }^{59}$, E. Bellet-Amalric ${ }^{59}$, F. Graner ${ }^{59}$, and L. Perino ${ }^{59}$

Interfacial Morphologies of Phospholipid Membrane Systems

B. Gaulin ${ }^{86}$, P. Mason ${ }^{23}$ and R. Epand ${ }^{86}$

Internal Organization of Peptide-Nucleic Acid Complexes Derived

From SANS

F. Schwarz ${ }^{20}$ and S. Krueger ${ }^{105}$

Interpretation of Specular and Off-Specular Reflectivity Data From

Patterned Thiahexaethyleneoxide Alkane Surfaces

N. F. Berk ${ }^{105}$, C. F. Majkrzak ${ }^{105}$, A. Munter ${ }^{105}$, J. Woodward ${ }^{107}$, C.W. Meuse ${ }^{107}$

A. L. Plant107, and S. Krueger ${ }^{105}$
In Situ Measurements of Melittin Insertion Into Hybrid Bilayer

Membranes

S. Krueger ${ }^{105}$, C. F. Majkrzak ${ }^{105}$, J. A. Dura ${ }^{105}$, A. L. Plant ${ }^{107}$, and C. W. Meuse ${ }^{107}$ Magnetic Field Alignment of Mixed Lipid Discotic Micelles

J. Katsaras ${ }^{23}$, R. Prosser ${ }^{74}$, S. Krueger ${ }^{105}$, and C. J. Glinka ${ }^{105}$

Membrane Active Peptides: Phase Transitions and Supramolecular

Assemblies of Peptides in Membranes

H. W. Huang ${ }^{145}$, W. Heller ${ }^{145}$, T. Harroun ${ }^{145}$, and L. Yang ${ }^{145}$

Molecular Dynamics of Solid-State Lysozyme as Affected by Glycerol and Water

A. M. Tsai ${ }^{105}$, D. A. Neumann ${ }^{105}$, and L. N. Belli8

Motion in Alpha-Lactalbumin and Alpha-Lactalbumin Molten Globule

Studied by Quasielastic Neutron Scattering

Z. Bu ${ }^{115}$, D. A. Neumann ${ }^{105}$, and C. C. Han ${ }^{115}$

Neutron Diffraction Studies of Peptides in Fluid Lipid Bilayers

S. White ${ }^{180}$ and K. Hristova ${ }^{180}$

Neutron Reflectivity Studies of Protein Adsorption/Desorption on the Surfaces of Self-Assembled Monolayers

M. D. Foster ${ }^{176}$, S. Petrash ${ }^{176}$, and C. F. Majkrzak ${ }^{105}$

Ordering in Protein/Polelectrolyte Multilayers

J. Rusling ${ }^{189}$ and $Y$. Lvov'10

Phase Behavior of Magnetically Alignable, Mixed Lipid 'Bicelles'

M.-P. Nieh ${ }^{137}$, J. Katsaras ${ }^{23}$, C. J. Glinka ${ }^{105}$, S. Krueger ${ }^{105}$, and R. S. Prosses ${ }^{74}$

Potential of Mean Force Between Neurofilaments at Different

Concentrations

S. Krueger ${ }^{105}$, M. Paulaits ${ }^{71}$, J. Hoh ${ }^{71}$, and S. Kumar ${ }^{137}$

Protein Adsorption at Solid/Liquid Interfaces

J. R. Lu'215, T. J. Su'215, and B. Green ${ }^{215}$

Quasielastic Neutron Scattering Study of the Dynamics of Water in Saccharide Solutions

A. M. Tsai ${ }^{105}$, M. Feeney ${ }^{140}$, P. G. Debenedetti ${ }^{140}$, and D. A. Neumann ${ }^{105}$

Rnase-A Dynamics by Time-of-Flight Neutron Scattering: A Case Study in Protein Dynamics and its Implications for Drug Design, Formulation and Delivery

A. M. Tsai ${ }^{105}$, T. J. Udovic ${ }^{105}$, J. R. D. Copley ${ }^{105}$ D. A. Neumann ${ }^{105}$, J. J. Rush ${ }^{105}$

M. Tarek ${ }^{200,105}$, D. Tobias ${ }^{180}$, and G. Gilliland ${ }^{107}$

SANS Study of Catechol Dioxygenases From Pseudomonas Putida SH1 S.-L Huang ${ }^{94}$, W.-H. Li94, and J. W. Lynn ${ }^{105}$

SANS Study of Colloidal Interactions of Proteins

O. Velev ${ }^{190}$, P. Hinderliter ${ }^{190}$, J. Silas ${ }^{190}$, and E. W. Kaler ${ }^{190}$

SANS Study of Cyclic-AMP-Dependent Protein Kinase

J. Zhao ${ }^{80}$, J. Trewhella80, D. Walsh ${ }^{179}$, and R. Bruschia179

SANS Study of the DNA/Flap Endonuclease Complex J. Trewhella ${ }^{80}, \mathrm{C}$. Kim ${ }^{80}$, and S. Gallagher ${ }^{80}$

SANS Study of the FerrodoxinTOL Component of Toluence Dioxygenase From Pseudomonas Putida F1

S.-L. Huang ${ }^{94}$, D. T. Gibson ${ }^{197}$, W.-H. Li94, and J. W. Lynn ${ }^{105}$

Solution Structure of Cyclic AMP Receptor Protein/DNA Complexes F. Schwarz ${ }^{20}$ S. K. Gregurick ${ }^{200}$, and S. Krueger ${ }^{105}$

Structural Investigation of the High Temperature Protonic Conductors C. Karmonik ${ }^{219}$, Q. Z. Huang ${ }^{199}, 105$, T. J. Udovic ${ }^{105}$, and J. J. Rush ${ }^{105}$

Structure of Hybrid Bilayer Membranes by Direct Inversion of Neutron

Reflectivity Data

C. Maikrzak' ${ }^{105}$, N. F. Berk ${ }^{105}$, C.W. Meuse ${ }^{107}$, V. Silin'107, A. L. Plant ${ }^{107}$, and S Krueger ${ }^{105}$

Structure of Intact Influenza Hemagglutinin Protein Containing the Viral Membrane Spanning Region

R. Blumenthal ${ }^{96}$, D. Remeta ${ }^{150}$, S. Durell ${ }^{96}$, and S. Krueger ${ }^{105}$

Structure of Microtubule-Based Cellular Motors Investigated by SANS R. Mendelson ${ }^{183}$, D. Stone ${ }^{183}$, and R. Hjelm ${ }^{80}$

Temperature Effect on the Structure and Activity of Catechol 2.3-Dioxygenase From Pseudomonas putida SH1: A Small Angle Neutron Scattering Study

S. L. Huang ${ }^{94}$, Y.-C. Hsu ${ }^{94}$, S. Y. Wu ${ }^{94}$, W. H. Li94, and J. W. Lynn ${ }^{105}$

Vectorially-Oriented Protein Monolayers: Neutron Reflectivity Studies J. K. Blasie 210 L. Kneller210 E. Nordgren ${ }^{210}$ J. Strzalka210 and A. Edwards'210 
A View of the Dynamical Changes in the Molten Globule-Native Folding Step of $\alpha$-Lactalbumin

Z. Bu115, D. A. Neumann'105, S.-H. Lee ${ }^{199,105}$, C. M. Brown'199,105, D. M. Engleman 233 , and C. C. Han ${ }^{115}$

\section{CHEMICAL PHYSICS OF MATERIALS}

A Quasi-Elastic and Inelastic Neutron Scattering Study of Hydrogen

in Zeolite

P. E. Sokol137, D. Narehood ${ }^{137}$, R. M. Dimeo ${ }^{105}$, J. DeWall137, and Y. Yoshida ${ }^{137}$

The Boson Peak in Solid-State Lysozyme

A. M. Tsaj105, C. L. Soles ${ }^{115}$, R. M. Dime0 ${ }^{105}$, R. L. Cappelletti105, and D. A Neumann 105

Cage Dynamics of $\left(R \mathrm{SiO}_{1.5}\right)_{n}\left(R=\mathrm{H}, \mathrm{CH}_{3}, \mathrm{C}_{2} \mathrm{H}_{3}\right.$ and $\left.n=8,10,12 \ldots\right)$

C. M. Brown ${ }^{199}, 105$, C. L. Soles ${ }^{115}$, and J. D. Lichtenhan ${ }^{54}$

Diffusion of $\mathrm{H}_{2}$ in $\mathrm{C}_{60}$

S. A. FitzGerald 128 , D. Sethna ${ }^{128}, Z$. Chowdhuri199. 105 , and D. A. Neumann ${ }^{105}$

Diffusion and Structural Relaxation in Viscous Sodium Disilicate A. Meyer ${ }^{163}$

Dynamics of Arsenic-Selenium Glasses

B. E. Schwickert ${ }^{178}$, R. L. Cappelletti105, and W. A. Kamitakahara105

Dynamics of $\mathrm{Cu}\left[\mathrm{N}(\mathrm{CN})_{2}\right]_{2}$. Pyrazine

C. M. Brown ${ }^{199} .105$ and J. L. Manson ${ }^{4}$

Dynamics of Filled Polymers

A. I. Nakatani115, R. D. Ivkov'105, P. Papanek210.105, and K. Kwan ${ }^{31}$

The Dynamics and Glass Transition in Ultra Thin Polymer Films

C. L. Soles ${ }^{115}$, R. M. Dimeo ${ }^{105}$, C. M. Brown ${ }^{199}{ }^{105}$, and W.-L. Wu115

The Dynamics of Hydrogen Bonded Guanidinium Based Materials A. Pivovar ${ }^{203}$, D. A. Neumann ${ }^{105}$, T. Yildirimn' ${ }^{105}$, C. M. Brown ${ }^{199.105}$, and M. Ward 203

Dynamics of Hydrogen in Laves Phase Hydrides

A. V. Skripov ${ }^{88}$, J. C. Cook ${ }^{199}, 105$, and T. J. Udovic ${ }^{105}$

The Dynamics of Hydrogen in Mesoporous Silica

T. J. Pinnavaia90, M. Gutmann"90, S. J. L. Billinge90, C. M. Brown ${ }^{199}$ 105, and

D. A. Neumann 105

The Dynamics of Hydrogen in Single-Walled Nanotubes C. M. Brown 199, 105 , T. Yildirim¹05, D. A. Neumann ${ }^{105}$, M. J. Heben ${ }^{145}$, T. Gennett97, A. C. Dillon97, J. L. Alleman'97, and J. E. Fischer210

Dynamics of Layered Silicates: Intercalated Water N. Wada ${ }^{170}$ and W. A. Kamitakahara ${ }^{105}$

Dynamics of Li-Doped Carbon Electrode Materials P. Papanek210, 105, J. E. Fischer210, and W. A. Kamitakahara105

Dynamics of Methane in Zeolites $4 \mathrm{~A}$ and $5 \mathrm{~A}$

R. Bell147, M. A. Green ${ }^{147}$, and C. M. Brown ${ }^{199.105}$

Dynamics of $\mathrm{Mn}\left[\mathrm{N}(\mathrm{CN})_{2}\right]_{2} \cdot$ pyrazine and its Deuterated Analogue C. M. Brown 199. 105 J. L. Manson ${ }^{4}$, and J. S. Miller220

Dynamics of Selenium-Arsenic-Bromine Glasses

R. L. Cappellettios, and B. E. Schwickert178

Dynamics of Water Absorbed in Epoxy

C. L. Soles ${ }^{115}$, R. M. Dime0 ${ }^{105}$, and W.-L. Wu ${ }^{115}$

Dynamics of Water in Zeolites

N. Wada170 and W. A. Kamitakahara ${ }^{105}$

The Effects of Confinement on Tunneling in Methyl lodide R. M. Dimeo ${ }^{105}$ and D. A. Neumann ${ }^{105}$

Effects of Solvent Damping on Side Chain and Backbone Contributions to the Protein Boson Peak

M. Tarek200.105 and D. J. Tobias 180

Evolution of the Structure of the $\mathrm{K}_{2} \mathrm{NiF}_{4}$ Phases of $\mathrm{La}_{2-\mathrm{x}} \mathrm{Sr}_{x} \mathrm{NiO}_{4+\delta}$ With Oxidation State: Octahedral Distortion and Phase Separation

$(0.2<x<1.0)$

J. E. Millburn ${ }^{134}$, M. A. Green ${ }^{147}$, M. J. Rosseinsky ${ }^{134}$, and D. A. Neumann ${ }^{105}$

First-Principles Calculations and Neutron Scattering

T. Yildirim ${ }^{105}$ and O. Gulseren210, 105

First-Principles Study of the Structure and Dynamics of $\mathrm{C}_{8} \mathrm{H}_{8}, \mathrm{Si}_{8} \mathrm{H}_{8}$ and $\mathrm{Ge}_{8} \mathrm{H}_{8}$ Molecules
C. Kilic ${ }^{14}$, T. Yildirim ${ }^{105}$, H. Mehrez ${ }^{14}$, and S. Ciraci14

Hydrogen Diffusion in $\mathrm{NbC}_{0.76} \mathrm{H}_{0.19}$

A. V. Skripov88, J. C. Cook ${ }^{199.105}$, and T. J. Udovic ${ }^{105}$

Hydrogen Dynamics in $\mathrm{K}_{3} \mathrm{MnH}_{5}$

G. Auffermann ${ }^{175}$, T. J. Udovic ${ }^{105}$, J. J. Rush ${ }^{105}$, and T. Yildirimos

Hydrogen Dynamics in $\mathrm{ZrBeH}_{1.5}$

T. J. Udovic ${ }^{105}$ and B. Hauback ${ }^{61}$

Hydrogen Trapping in TaN $\mathrm{N}_{0.006} \mathrm{H}_{0.003}$

M. Heene ${ }^{30}$, H. Wip 30 , T. J. Udovic ${ }^{105}$, and J. J. Rush ${ }^{105}$

Inelastic Neutron Scattering Study of Al Charged With Hydrogen

T. J. Udovic ${ }^{105}$, J. J. Rush ${ }^{105}$, C. Buckley ${ }^{179}$, and H. K. Birnbaum' ${ }^{195}$

Inelastic Neutron Scattering Study of Rare Earth Trihydrides and Trideuterides

T. J. Udovic ${ }^{105}$, J. J. Rush'105, and Q. Z. Huang ${ }^{199}, 105$

Investigation of Slow Motion Dynamics in Selective Adsorption

H. Taub204, D. Fuhrmann204, L. Criswell204, A. Diamu204, K. W. Herwig4, F. Y.

Hansen ${ }^{162}$, and R. M. Dimeo ${ }^{105}$

Low Energy Dynamics of Alkali Silicate Glasses

J. Toulouse ${ }^{78}$ and D. A. Neumann ${ }^{105}$

Low Energy Dynamics in Polycarbonate Copolymers

C. L. Soles ${ }^{115}$, R. M. Dime0 ${ }^{105}$ A. F. Yee, and W.-L. Wu ${ }^{115}$

Low Energy Phonons in Nanocrystals of $\mathrm{Ni}_{3} \mathrm{Fe}$

A. Yue ${ }^{18}$, B. Fultz'18, H. N. Frase ${ }^{18}$, J. L. Robertson ${ }^{127}$, and R. M. Dimeo ${ }^{105}$

Neutron Scattering Studies of the Dynamics of Hydrofluorocarbons Encaged in $\mathrm{NaX}$ and $\mathrm{NaY}$ Zeolites

C. Karmonik219, N. C. Maliszewskyj!05, T. J. Udovic ${ }^{105}$, J. J. Rush ${ }^{105}$, M. K.

Crawford ${ }^{33}$, D. R. Corbin², and R. R. Cavanagh ${ }^{119}$

Neutron Scattering Studies of Hydration Reaction of Tricalcium

Silicate and Portland Cement

J. J. Thomas ${ }^{124}$, S. A. FitzGerald128, D. A. Neumann ${ }^{105}$, and R. A. Livingston ${ }^{39}$

Neutron Scattering Studies on the Vibrational Excitations and the

Structure of Ordered Niobium Hydrides

B. Hauer ${ }^{44}$, E. Jansen ${ }^{44}$, W. Kockelmann ${ }^{44}$, W. Schafer ${ }^{44}$, D. Richter ${ }^{44}$, R.

Hempelmann ${ }^{174}$, T. J. Udovic ${ }^{105}$, and J. J. Rush ${ }^{105}$

Neutron Scattering Study of the Role of Diffusion in the Hydration

Reaction of Tricalcium Silicate

S. A. FitzGerald ${ }^{128}$, J. J. Thomas, D. A. Neumann ${ }^{105}$, and R. A. Livingston ${ }^{39}$

Neutron Scattering Study of the Structure of $\mathrm{Na}_{2} \mathrm{C}_{60}$ as a Function of

Pressure and Temperature

T. Yildirim¹05, D. A. Neumann ${ }^{105}$, S. F. Trevino9. 105 , and J. E. Fischer ${ }^{210}$

Neutron Scattering Study of the Dynamics of Hydrogen and Deuterium Dissolved in Crystalline $\mathrm{Pd}_{9} \mathrm{Si}_{2}$

T. J. Udovic 105, Q. Z. Huang ${ }^{199}, 105$, J. J. Rush ${ }^{105}$, C. Karmonik ${ }^{219}$, Y.

Andersson ${ }^{146}$, and T. B. Flanagan 221

Neutron Scattering Study of the Freeze-Thaw Process in Portland Cement

J. C. McLaughlin ${ }^{124}$, R. L. Gress ${ }^{208}$, D. A. Neumann ${ }^{105}$, and R. A. Livingston 39

Neutron Scattering Study of the Liquid-Glass Transition in Trimethylheptane

G. Q. Shen ${ }^{24}$, J. Toulouse ${ }^{78}$, S. Beaufils ${ }^{213}$, B. Bonello211, Y. H. Hwang 24, P.

Finke $^{178}$, J. Hernandez 24 , M. Bertault213, M. Maglione ${ }^{177}$, C. Ecolivet' ${ }^{213}$, and H.

Z. Cummins 24

Neutron Spectroscopy of Single-Walled Nanotubes

P. Papanek $k^{210}, 105$, J. E. Fischer ${ }^{210}, Z$. Benes ${ }^{210}$, and A. Clay210

Neutron Vibrational Spectroscopy and Hopping Dynamics of $H$ and $D$ in $\mathrm{Pd}_{3} \mathrm{P}_{0.8} \mathrm{H}(\mathrm{D})_{x}$

T. J. Uddovic ${ }^{105}$, Q. Z. Huang 199.105 , and Y Andersson 221

Neutron Vibrational Spectroscopy of Organic Materials

B. S. Hudson ${ }^{159}$, C. Middleton ${ }^{159}$, T. Jenkins ${ }^{159}$, Y. Kuzmicheva90, S. Baronov90,

and C. M. Brown ${ }^{199}, 105$

Neutron Vibrational Spectroscopy of $\mathrm{Sr}_{2} \mathrm{IrH}_{5}$ and $\mathrm{Sr}_{2} \mathrm{RuH}_{6}$

T. J. Udovic ${ }^{105}$ and R. O. Moyer ${ }^{17}$

Phonon Densities of States of $\gamma$-Ce and $\delta$-Ce Measured by Inelastic Neutron Scattering

M. E. Manley ${ }^{18,80}$, R. J. McQueeney ${ }^{80}$, J. L. Robertson ${ }^{127}$, B. Fultz'18, and D.

A. Neumann 105 
Phonon Density of States in $\mathrm{Sc}_{2}\left(\mathrm{WO}_{4}\right)_{3}$

C. Broholm ${ }^{71}, 105$, C. Ulrich ${ }^{71}$, G. Gasparovic ${ }^{71}$, and C. M. Brown ${ }^{199,105}$

Phonon Density of States of U-238

M. E. Manley18, 80, R. A. Robinson ${ }^{80}$, C. M. Brown ${ }^{199.105}$, and B. Fultz' ${ }^{18}$

Polarization and Structural Transitions of Irradiated Vinylidene

Fluoride-Trifluoroethylene Copolymer

E. Balizer102, A. S. DeReggi115, D. A. Neumann ${ }^{105}$, and F. B. Bateman ${ }^{111}$

Preferential Isotopic Site Occupation in Rare-Earth Hydrides

T. J. Udovic ${ }^{105}$, Q. Z. Huang ${ }^{199,} 105$, and J. J. Rush ${ }^{105}$

Pressure-Induced Interlinking of Carbon Nanotubes

T. Yildirim ${ }^{105}, 0$. Gulseren²10. 105, and S. Ciraci14

Proton Diffusion in $\mathrm{Cs}_{2}\left(\mathrm{HSO}_{4}\right)\left(\mathrm{H}_{2} \mathrm{PO}_{4}\right)$

S. M. Haile ${ }^{18}$, C. Chisolm ${ }^{18}$, Z. Chowdhuri199, 105, T. Yildirimo ${ }^{105}$, and D. A.

Neumann ${ }^{105}$

Proton Diffusion in $\mathrm{MH}\left(\mathrm{SO}_{4}\right)_{1 \cdot x}\left(\mathrm{SeO}_{4}\right)_{x}, \mathrm{M}=\mathrm{Rb}, \mathrm{Cs}$

T. Yildirim ${ }^{105}$, Z. Chowdhuri'199.105, S. M. Haile ${ }^{18}$, C. Chisolm ${ }^{18}$, D. A

Neumannn ${ }^{105}$, and T. J. Udovic ${ }^{105}$

Proton Dynamics in Brown Millerite-Structured $\mathrm{Ba}_{2} \mathrm{In}_{2} \mathrm{O}_{5+\delta} \mathrm{H}_{25}$ : A

Combined Inelastic Neutron Scattering and Theory Study

T. Yildirimin ${ }^{105}$ D. A. Neumann ${ }^{105}$, and T. J. Udovic ${ }^{105}$

Proton Dynamics in Hydrated Nafions

T. J. Udovic ${ }^{105}$, S. F. Trevino ${ }^{9}$ 105, S. K. Young ${ }^{6}$, M. K. Crawford ${ }^{33}$, and Q. Sun ${ }^{33}$

Proton Dynamics in Strontium Cerate and Zirconate Ceramics

T. Yildirim ${ }^{105}$, T. J. Udovic ${ }^{105}$, C. Karmonik ${ }^{219}$, J. J. Rush ${ }^{105}$, and R. L. Paul106

Quantum Dynamics of Hydrogen in a Confined Potential

T. Yildirim ${ }^{105}$ and A. B. Harris210

The Role of Particle Size in the Hydration Reaction of Tricalcium

Silicate

J. C. McLaughlin124, D. A. Neumann ${ }^{105}$, and R. A. Livingston ${ }^{39}$

Rotational Dynamics of $\mathrm{C}_{60}$ in Superconducting $\mathrm{K}_{3} \mathrm{Ba}_{3} \mathrm{C}_{60}$

S. Margadonna ${ }^{216}$, W. Z. Lij ${ }^{216}$, K. Prassides ${ }^{216}$, and D. A. Neumann ${ }^{105}$

Sorption of Gases Into Single-Walled Carbon Nanotubes

T. Yildirim ${ }^{105}$, C. M. Brown ${ }^{199}, 105$, D. A. Neumann105, and J. E. Fischer ${ }^{210}$

Structural Ordering and Dynamics in $\operatorname{LaH}(D)_{3-x}$

T. J. Udovic ${ }^{105}$, Q. Z. Huang ${ }^{199}$, 105, C. Karmonik219, and J. J. Rush'105

Structural Symmetry of $\mathrm{YD}_{3}$ Epitaxial Thin Films

T. J. Udovic ${ }^{105}$, Q. Z. Huang ${ }^{199}, 105$, R. W. Erwin ${ }^{105}$, B. Hjorvarsson 226 , and R.

C. C. Ward226

Structure and Dynamics of Amorphous Carbon

P. Papanek210, 105, W. A. Kamitakahara105, and J. E. Fischer ${ }^{210}$

Structure and Dynamics of Hydrogen in $\mathrm{Na}_{x} \mathrm{C}_{60}$

S. A. FitzGerald ${ }^{128}$, T. Yildirim ${ }^{105}$, L. J. Santodonato ${ }^{105}$, and D. A. Neumann ${ }^{105}$

Structure and Dynamics of Thallium and Tellurium Containing Low

Thermal-Conductivity Materials

B. Wolfing ${ }^{82}$, C. M. Brown ${ }^{199}, 105$, and B. Battlog82

Structure and Dynamics of an Ultra Hard Carbon

W. A. Kamitakahara105, S. F. Trevino9, 105, and S. G. Buga ${ }^{67}$

Time-Temperature Superposition of Structural Relaxation in a Viscous

Metallic Liquid

A. Meyer ${ }^{163}$, R. Busch ${ }^{18}$, and H. Schober ${ }^{59}$

Two and Three Dimensional Rotons in Ordered Porous Media

P. E. Sokol137, D. Narehood137, and R. M. Dimeo ${ }^{105}$

Vibration of Dodecahedrane: A Combined Neutron Scattering and

Theoretical Study

T. Yildirm ${ }^{105}$, B. S. Hudson ${ }^{159}$ and C. Middleton ${ }^{159}$

Vibrational Densitites of States for Cubic and Hexagonal Boron Nitride

W. A. Kamitakahara ${ }^{105}$, D. A. Neumann ${ }^{105}$, G. Doll48, B Sweeting ${ }^{48}$, and A. W.

Moore ${ }^{1}$

Water Dynamics in Monosaccharide Solutions

M. Feeney ${ }^{140}$, C. M. Brown ${ }^{199,} 105$, A. M. Tsaj105, D. A. Neumann ${ }^{105}$, and P.

Debenedetti 140

Water Dynamics in Saturated MCM-41

F. Mansour 225, R. M. Dimeo ${ }^{105}$, and H. Peemoeller225
X-ray Diffraction Study of the Hydration Reaction in Tricalcium Silicate J. C. McLaughlin ${ }^{124}$, S. A. FitzGerald ${ }^{128}$, D. A. Neumann ${ }^{105}$, and R. A. Livingston ${ }^{39}$

\section{INSTRUMENTATION}

Assembly and Testing of the Filter Analyzer Spectrometer A. W. Clarkson ${ }^{105}$, D. J. Pierce ${ }^{105}$ J. D. Reardon ${ }^{105}$, S. Slifer ${ }^{105}$, and G. M. Baltic ${ }^{105}$

Data Acquisition and Analysis Software for the Backscattering Spectrometer

N. C. Maliszewskyj ${ }^{105}$ and R. M. Dimeo ${ }^{105}$

Data Acquisition and Analysis Software for the Filter Analyzer Neutron Spectrometer and the Ultra Low Angle Neutron Spectrometer N. C. Maliszewskyj ${ }^{105}$

Data Acquisition Electronics for the DCS J. B. Ziegler105

Data Acquisition Software for the DCS N. C. Maliszewskyj ${ }^{105}$

Data Analysis Software for the Fermi Chopper Spectrometer T. Yildirim ${ }^{105}$ and C. M. Brown ${ }^{199,} 105$

Data Reduction and Analysis Software for the DCS J. C. Cook ${ }^{105}$ and J. R. D. Copley ${ }^{105}$

Design of the Next Generation Cold Neutron Triple Axis Spectrometer C. W. Brocker ${ }^{199.105}$, C. L. Broholm¹. 105, and Y. Qiü1

Design of the Filter Analyzer Spectrometer D. J. Pierce ${ }^{105}$ and J. T. Kenney ${ }^{105}$

Design of Infrastructure Improvements to the Disk Chopper Spectrometer C. W. Brocker ${ }^{199,} 105$ and J. Bailey ${ }^{105}$

Design and Performance Testing of an Active Double Focusing

Monochromator System

S. A. Smee ${ }^{199}, 105$, J. D. Orndorff71, G. A. Scharfstein ${ }^{71}$, P. C. Brand ${ }^{105}$, and C. L. Broholm 71, 105

Design and Performance Testing of the DCS Data Acquisition System Electronics J. B. Ziegler ${ }^{105}$, N. C. Maliszewskyj ${ }^{105}$, and P. Klosowski105

Design of the Perfect Crystal SANS Diffractometer J. J. Moyer ${ }^{105}$ and J. T. Kenney ${ }^{105}$

Design and Testing of Vertically Polarized Heusler Alloy Monochromator/Analyzer C. P. Adams ${ }^{199,105}$ and J. W. Lynn'105

Development of a Bent Silicon Crystal Monochromator and Other Intensity Enhancements for the Diffractometer for Residual Stress Measurement, Texture Determination and Single Crystal Diffraction H. J. Prask ${ }^{105}$, T. Gnäupel-Herold ${ }^{199} .105$, J. G. LaRock ${ }^{105}$, and M. P. Popovici204

Development of a Dance Floor and Air Pad System for the Next Generation Neutron Scattering Instruments M. Murbach ${ }^{199} 105$, D. Pierce ${ }^{105}$, C. W. Wrenn ${ }^{199 .}{ }^{105}$, and P. C. Brand ${ }^{105}$

Development and Design of the Next-Generation Triple-Axis Spectrometer C. W. Wrenn ${ }^{199 .} 105$, M. Murbach ${ }^{199}$, 105, P. C. Brand105, and J. W. Lynn ${ }^{105}$

Development and Installation of a Leak-Tight, Top-Loading, Horizontal Field Superconducting Magnet

D. C. Dender ${ }^{105}$, R. W. Erwin ${ }^{105}$, and S. M. Choi ${ }^{199.105}$

Development of Low Activation Sample Holders for use at Small Angle Neutron Scattering and Spin Echo Spectrometry Instrumentation D. B. Fulford 105

Development of a Radially-Focussed Polarizing Supermirror Analyzer Array J. J. Moyer ${ }^{105}$, P. C. Brand ${ }^{105}$, and N. Rosov 105

Development of a Robust Static Thermal Switch for Fixed Sample Environment Temperatures From 15-650K L. Santodonato ${ }^{105}$ and P. C. Brand ${ }^{105}$

Development of the HFBS Doppler Drive R. M. Dime0 ${ }^{105}$, P. C. Brand ${ }^{105}$, and M.J. Rinehart ${ }^{105}$ 
Development of a Vacuum Rated Preamplifier/Amplifier/-Discriminator for ${ }^{3} \mathrm{He}$ Neutron Detectors

J. B. Ziegler ${ }^{105}$

The Disk Chopper Time-of-Flight Spectrometer (DCS)

J. R. D. Copley ${ }^{105}$, J. C. Cook ${ }^{199}, 105$, F. B. Altorfer ${ }^{136}$, G. M. Baltic ${ }^{105}$, P. Brand ${ }^{105}$ C. W. Brocker ${ }^{199},{ }^{105}$, D. L. Clemn ${ }^{105}$, W. R. Clow ${ }^{105}$, W. E. Dickerson ${ }^{105}$, M. C English ${ }^{105}$, P. D. Gallagher ${ }^{105}$, A. E. Heald105, P. Klosowski105, W. C. Knill105, D. L. Kulp ${ }^{105}$, H. P. Layer ${ }^{105}$, N. C. Maliszewskyj ${ }^{105}$, D. J. Pierce ${ }^{105}$, J. Räbiger ${ }^{44}$, J. D. Reardon ${ }^{105}$, M. J. Rinehart'105, I. G. Schröder ${ }^{105}$, L. A. Shuman ${ }^{105}$, S. Slifer ${ }^{105}$, T. T. Thai ${ }^{105}$, P. J. Tobin ${ }^{105}$, and J. B. Ziegler ${ }^{105}$

Dy-Foil Detection of Neutrons

Y.-T. Cheng ${ }^{104,108}$, B. H. Toby ${ }^{105}$, and T. Gnäupel-Heroid 199,105

Evaluation of New Neutron Area Detectors Y. T. Cheng 104,106

The Filter Analyzer Spectrometer

D. A. Neumann ${ }^{105}$, P. Papanek210, 105, J. E. Fischer210, J. J. Rush'105, M. L. Klein ${ }^{210}$

A. C. Cheetham ${ }^{184}$, and T.J. Udovic ${ }^{105}$

Gas Handling System for the DCS

P. Brand ${ }^{105}$, C. W. Brocker ${ }^{105}$, M. C. English ${ }^{105}$, and S. Slifer ${ }^{105}$

A Helium Cooling System for the Electromagnetic Field Correction

Coils of the Neutron Spin Echo Spectrometer

P. C. Brand ${ }^{105}$, P. D. Gallagher ${ }^{105}$, D. B. Fulford ${ }^{105}$, and W. R. Clow ${ }^{105}$

Hydrogen Cold Source Development

R. E. Williams ${ }^{105}$, P. A. Kopetka105, and J. M. Rowe ${ }^{105}$

Infrastructure Improvements in the Reactor Confinement Building D. B. Fulford ${ }^{105}$, M. C. English ${ }^{105}$, and P. D. Gallagher ${ }^{105}$

In Situ Real-Time Neutron Beam Imaging

K. T. Forstner ${ }^{105}$ and N. C. Maliszewskyj] ${ }^{105}$

Installation of Dedicated Crystal Alignment Spectrometer at BT-7

Beamline for Construction of FANS Double Focusing Copper

Monochromator

K. T. Forstner ${ }^{105}$ and A. W. Clarkson ${ }^{105}$

Installation of a New Heusler Alloy Polarizing Analyzer for BT-2

K. T. Forstner ${ }^{105}$, J. A. Borchers ${ }^{105}$, and J. W. Lynn ${ }^{105}$

Installation of the Sample Chamber, Flight Chamber and Detector

Assembly for the DCS

C. W. Brocker ${ }^{199}, 105$, M. J. Rinehart ${ }^{105}$, G. M. Baltic ${ }^{105}$, and S. Slifer ${ }^{105}$

Installation and Testing of the New Thermal Beam Tube Shutter Design

Using a Remote Actuated Cast System

J. J. Moyer105, C. W. Wrenn ${ }^{199}{ }^{105}$, P. C. Brand ${ }^{105}$, A. W. Clarkson ${ }^{105}$, G. M.

Baltic ${ }^{105}$, D. L. Clem ${ }^{105}$, M. J. Rinehart105, and S. Slifer ${ }^{105}$

MBE Chamber for In Situ Neutron Scattering: Instrument Development J. A. Dura105

Mechanical Solution to Reduce Velocity Selector Vibrations P. C. Brand ${ }^{105}$ and J. Bailey ${ }^{105}$

Modular Electronics Package for Stepper Motor Operation

D. L. Kulp ${ }^{105}$, M. C. English ${ }^{105}$, P. Klosowski105, and N. C. Maliszewskyjj ${ }^{105}$

Monte Carlo Methods for Nuclear Applications

R. E. Williams ${ }^{105}$

Neutron Scattering Properties and Materials Properties Testing of the

Null Scattering Titanium Alloy to be Used for High Pressure Cells

S. F. Trevino9. 105, K. T. Forstner ${ }^{105}$, and R. Fields ${ }^{113}$

The NIST Neutron Spin Echo Spectrometer (NSE)

N. Rosov ${ }^{105}$, S. Rathgeber ${ }^{199,}{ }^{105}$, and M. Monkenbusch ${ }^{44}$

The Perfect Crystal SANS Diffractometer

J. G. Barker ${ }^{105}$, J. J. Moyer ${ }^{105}$, and C. J. Glinka105

Reassessment of NG-3 Optical Filter Performance Based on

Supermirror Reflectivity Data J. C. Cook 199.105

SANS Polarization Analysis With Nuclear-Spin-Polarized ${ }^{3} \mathrm{He}$ T. R. Gentile ${ }^{111}$, G. L. Jones' ${ }^{111}$, A. K. Thompson ${ }^{111}$, J. G. Barker ${ }^{105}$, C. J. Glinka ${ }^{105}$, B. Hammouda ${ }^{105}$, and J. W. Lynn ${ }^{105}$

Search for Effects on Neutron Transmission Due to Multiple Reflection by Glass Capillary Walls

R. E. Beneson ${ }^{106,232}$, H. H. Chen-Mayer ${ }^{106}$, and J. W. Lynn ${ }^{105}$
TANQENS-A New Utility for the Analysis of Quasielastic Neutron

Scattering Data

T. Yildirim 105

\section{NEUTRON PHYSICS}

Accelerated Testing for Neutron Induced Soft Errors in SRAM Chips A. K. Thompson ${ }^{111}$ and J. F. Ziegler ${ }^{106}$

Accurate Determination of Neutron Capture Flux M. S. Dewey ${ }^{111}$, M. Arif111, D. M. Gilliam¹1, J. S. Nico ${ }^{111}$, W. M. Snow ${ }^{58}$, J. Pauwels ${ }^{65}$, and R. Scott85

Benchmark Measurements and Calculations of Neutron Transport D. M. Gilliam¹1, M. S. Dewey'111, J. S. Nico0 ${ }^{111}$, C. M. Eisenhauer ${ }^{111}$, J. A. Grund ${ }^{111}$, and $H$. Gerstenberg ${ }^{5}$

Certified Neutron Fluence Standards From the Cavity Fission Source J. M. Adams ${ }^{111}$ and J. A. Grund ${ }^{111}$

Defined-Geometry Alpha Counting D. M. Gilliam ${ }^{111}$ and J. S. Nico'11

Determination of the Neutron Lifetime M. S. Dewey ${ }^{111}$, J. S. Nico ${ }^{111}$, D. M. Gilliam ${ }^{111}$, W. M. Snow ${ }^{58}$, and P. R. Huffman'11

Highly Accurate Neutron Wavelength Measurements M. S. Dewey ${ }^{111}$, K. J. Coakley ${ }^{118}$, W. M. Snow ${ }^{58}$, M. Arifi11, D. L. Jacobson ${ }^{111}$, and S. A. Werner204

Intercomparison of NIST and PNL Calibration Facilities A. K. Thompson ${ }^{111}$, R. B. Schwartz"11, J. McDonald ${ }^{11}$, and M. Murphy'

LASER Polarization of ${ }^{3} \mathrm{He}$ for Neutron Spin Filters and Medical MRI A. K. Thompson ${ }^{111}$, T. R. Gentile ${ }^{111}$, M. S. Dewey 111 , G. L. Jones ${ }^{50}$, F. E. Wietfeldt111, 51, W. M. Snow ${ }^{58}$, and D. R. Rich ${ }^{11}$

Neutron Calorimetry W. M. Snow58, Z. Chowdhuri199, 105, J. S. Nico ${ }^{111}$, M. S. Dewey'111, and G. Hansen $^{58}$

Neutron Fluence Rate Measurements

D. M. Gilliam¹1, J. S. Nico ${ }^{111}$, and J. M. Adams ${ }^{111}$

Neutron Interferometry and Optics

M. Arif111, D. L. Jacobson ${ }^{111}$, R. Clothier ${ }^{13}$, A. K. Thompson ${ }^{111}$ S. A. Werner204 A. loffe ${ }^{204}$, 111, A. Zeilinger ${ }^{196}$, K. Raum ${ }^{206}$, B. Schillinger ${ }^{206}$, C. Rausch ${ }^{206}$, and W. Richards 85

Neutron Radiography/Tomography T. R. Gentile ${ }^{111}$, M. Arif111, and D. L. Jacobson ${ }^{111}$

Parity Non-Conserving Neutron Spin Rotation B. Heckel224, D. Markoft224, E. Adelberger224, F. E. Wietfeldt ${ }^{111}, 51$, and P. R. Huffman ${ }^{11}$

Quality Assurance Checks on Masses and Impurities in Neutron Dosimeters for NRC Reactor Pressure Vessel Embrittlement Surveillance

J. M. Adams 111 D. M. Gilliam ${ }^{111}$, and J. A. Grundl111

Response of Albedo Dosimeters Versus Distance From a Neutron Source

R. B. Schwartz'11 and A. K. Thompson ${ }^{111}$

Study of Time Reversal Invariance in Neutron Beta Decay J. Wilkerson ${ }^{229}$, J. S. Nico ${ }^{111}$, R. G. H. Robertson ${ }^{178}$, S. Freedman ${ }^{178}, A$ Garcia $^{209}$, K. Coulter ${ }^{18}$, M. S. Dewey ${ }^{111}$, A. K. Thompson ${ }^{111}$, B. Fujikawa ${ }^{178}$, G. L. Jones ${ }^{111,58}$, J. M. Adams ${ }^{111}$, F. E. Wietfeldt111,51, L. Lising ${ }^{178}$, and T. Steiger ${ }^{224}$

Trapping of Ultra Cold Neutrons

J. M. Doyle ${ }^{51}$, S. K. Lamoreaux ${ }^{80}$, R. Golub ${ }^{49}$, M. S. Dewey ${ }^{111}$, P. R. Huffman ${ }^{111}$

C. R. Brome ${ }^{51}$, C. E. H. Mattoni51, D. N. McKinsey51, K. Alvine ${ }^{51}$, S. Dzhosynk ${ }^{51}$,

L. Yan ${ }^{51}$ g, K. Coakley ${ }^{111}$, G. Yang ${ }^{111}$, and A. K. Thompson ${ }^{111}$

Utilization of the Materials Dosimetry Reference Facility-Tests of New IRMM ${ }^{237} \mathrm{~Np}$ and ${ }^{238} \mathrm{U}$ Fast Neutron Dosimeters

J. M. Adams ${ }^{111}$, J. A. Grund $1{ }^{111}$, C. M. Eisenhauer ${ }^{111}$, D. M. Gilliam ${ }^{111}$, and P Simpson ${ }^{18}$ 


\section{MATERIALS ANALYSIS}

Analytical Applications of Cold Neutrons

H. H. Chen-Mayer ${ }^{106}$, R. Demiralp ${ }^{106}$, R. R. Greenberg ${ }^{106}$, G. P. Lamaze ${ }^{106}$, J.

K. Langland ${ }^{106}$, R. M. Lindstrom ${ }^{106}$, E. A. Mackey ${ }^{106}$, D. F. R. Mildner ${ }^{106}$, R. L.

Paul106, and V. A. Sharov232

Application of Radioisotope-Induced X-ray Emission to the

Identification of Lead and Other Elements in Ceramic Glazes and

Housewares

D. L. Anderson ${ }^{42}$ and W. C. Cunningham ${ }^{42}$

Archeological Applications of NAA

R. Bishop ${ }^{155}$ and M. J. Blackman ${ }^{106}$

A Bender-Focuser for Prompt Gamma Activation Analysis

E. A. Mackey ${ }^{106}$, H. H. Chen-Mayer ${ }^{106}$, R. M. Lindstrom ${ }^{106}$, D. F. R. Mildner ${ }^{106}$,

R. L. Paul ${ }^{106}$, Q.-F. Xiao 222 , and V. A. Sharov232

Bio-Analytical and Specimen Bank Research

R. R. Greenberg ${ }^{106}$, J. K. Langland ${ }^{106}$, E. A. Mackey ${ }^{106}$, B. J. Porter ${ }^{106}$, and R.

L. Zeisler 106

Certification of Standard Reference Materials by Neutron Activation

Analysis

D. A. Becker ${ }^{106}$, R. Demiralp ${ }^{105}$, R. R. Greenberg ${ }^{106}$, R. M. Lindstrom ${ }^{106}$, E. A. Mackey ${ }^{106}$, and R. L. Zeisler ${ }^{106}$

Characterization of Sources and Fluxes of Particulate Pollutants

Depositing to Great Waters

P. F. Caffrey ${ }^{199}$, A. E. Suarez ${ }^{199}$, and J. M. Ondov ${ }^{199}$

Characterization of Submicrometer Aerosol Particles

J. M. Ondov' ${ }^{199}$, S. F. Heller-Zeisler ${ }^{199}$, and R. L. Zeisler ${ }^{106}$

Determination of Hydrogen by PGAA

R. R. Greenberg ${ }^{106}$, R. M. Lindstrom ${ }^{106}$, and R. L. Paul106

Determination of Phosphorus via RNAA with Beta Counting R. L. Paul106

Evaluation of Accuracy and Precision in INAA of Botanical Materials D. A. Becker ${ }^{106}$

Evaluation of Errors and Interferences in NAA

D. A. Becker ${ }^{106}$, M. J. Blackman ${ }^{155}$, R. R. Greenberg ${ }^{106}$, R. M. Lindstrom ${ }^{106}$, and R. L. Zeisler ${ }^{106}$

Focusing Methods for Radiography and Topography

H. H. Chen-Mayer ${ }^{106}$, D. F. R. Mildner ${ }^{106}$, K. M. Podurets ${ }^{75}$ V. A. Sharov232, and S. S. Shilstein ${ }^{75}$

High Sensitivity Gamma-Ray Spectrometry

R. M. Lindstrom ${ }^{106}$ and G. P. Lamaze ${ }^{106}$

Homogeneity and Composition of Small Solid Samples R. L. Zeisler 106

Hydrogen Detection in Industrial Materials by Incoherent Neutron

Scattering

H. H. Chen-Mayer ${ }^{106}$, V. V. Kvardakov ${ }^{75}$ D. F. R. Mildner ${ }^{106}$, V. A. Somenkov ${ }^{75}$, and D. Kozlenko ${ }^{72}$

Improvements to INAA Methodology

D. A. Becker ${ }^{106}$, R. Demiralp ${ }^{106}$, R. R. Greenberg ${ }^{106}$, R. M. Lindstrom ${ }^{106}$, E. A.

Mackey ${ }^{106}$, and R. L. Zeisler ${ }^{106}$

Improvements to PGAA Methodology

D. L. Anderson 42, R. M. Lindstrom ${ }^{106}$, E. A. Mackey ${ }^{106}$, and R. L. Paul106

Measurement of Lithium Concentrations in Battery Materials with Cold Neutron Depth Profiling

G. P. Lamaze ${ }^{106}$, H. H. Chen-Mayer ${ }^{106}$, D. A. Becker ${ }^{106}$, A. Gerouki ${ }^{172}$, F.

Vereda $^{172}$, N. Clay ${ }^{172}$, P. Zerigian ${ }^{172}$, and T. Haas ${ }^{172}$
Multielement Analysis of Foods and Related Materials by NAA

D. L. Anderson 42 and W. C. Cunningham ${ }^{42}$

Neutron Absorption Measurements Using Converging Beams

H. H. Chen-Mayer ${ }^{106}$, E. A. Mackey ${ }^{106}$, D. F. R. Mildner ${ }^{106}$, and R. L. Paul106

Neutron Beam Spatial Distributions Using Imaging Plate Technology

H. J. Bahre ${ }^{45}$, H. H. Chen-Mayer ${ }^{106}$, Y.-T. Cheng ${ }^{104}{ }^{106}$, D. F. R. Mildner ${ }^{106}$, and

V. A. Sharov232

Neutron Depth Profiling of AIN Thin Films

H. H. Chen-Mayer106, G. P. Lamaze ${ }^{106}$, and S. McGuire ${ }^{28}$

Neutron Depth Profiling of Sulfur in CVD Diamonds

H. H. Chen-Mayer ${ }^{106}$, G. P. Lamaze ${ }^{106}$, L. Pilione ${ }^{137}$, and B. Weiss ${ }^{212}$

Nitrogen Profiling of Thin Titanium Nitride Films

G. P. Lamaze ${ }^{106}$, H. H. Chen-Mayer ${ }^{106}$, N. Cox ${ }^{68}$, and E. B. Steel ${ }^{119}$

Neutron Focusing for Analytical Chemistry

H. Chen-Mayer ${ }^{106}$, G. P. Lamaze ${ }^{106}$, J. K. Langland ${ }^{106}$, R. M. Lindstrom ${ }^{106}$, E. A.

Mackey ${ }^{106}$, D. F. R. Mildner ${ }^{106}$, V. A. Sharov232 and J. R. Swider ${ }^{119}$

Neutron Distribution Measurements by Neutron Induced

Autoradiography

Z. En222, J. S. Brenizer222, and D. A. Becker ${ }^{106}$

Neutron Dosimetry for Instrument Development

R. M. Lindstrom 106

Neutron Transmission Through Tapered Capillaries

H. H. Chen-Mayer ${ }^{106}$, D. F. R. Mildner ${ }^{106}$, and V. A. Sharov232

New Developments in Monitor Activation Analysis

G. P. Lamaze ${ }^{106}$, R. M. Lindstrom ${ }^{106}$, and R. L. Paul106

New Developments in NDP

H. H. Chen-Mayer ${ }^{106}$, G. P. Lamaze ${ }^{106}$, and J. K. Langland ${ }^{106}$

Quality Assurance Improvements for NAA

D. A. Becker ${ }^{106}$, R. Demiralp ${ }^{106}$, R. R. Greenberg ${ }^{106}$, R. M. Lindstrom ${ }^{106}$, E. A Mackey ${ }^{106}$, and R. L. Zeisler ${ }^{106}$

Quality Assurance Programs

D. L. Anderson 42 and W. C. Cunningham ${ }^{42}$

Reactor Characterization for NAA

D. A. Becker ${ }^{106}$

Redesign of the VT-5 Thermal Neutron Prompt Gamma-Ray Activation Analysis Facility

E. A. Mackey ${ }^{106}$, R. M. Lindstrom ${ }^{106}$, D. L. Anderson ${ }^{42}$, P. J. Liposky105, G. D. Reilly ${ }^{105}$, D. R. Brown ${ }^{114}$, and M. A. Suthar ${ }^{105}$

Radionuclides in Foods

D. L. Anderson ${ }^{42}$ and W. C. Cunningham ${ }^{42}$

Revalidation of Food SRMs

D. L. Anderson 42 and W. C. Cunningham ${ }^{42}$

Size Distribution and Deposition of Toxic Elements to Lake Michigan, by Size and by Source

P. F. Caffrey ${ }^{199}$ and J. M. Ondovi99

Test of a Novel High Sensitivity Neutron Detector

B. Feller ${ }^{125}$, H. H. Chen-Mayer ${ }^{106}$, and G. Lamaze ${ }^{106}$ 


\section{AFFILIATIONS:}

Advanced Ceramics Corporation AEA Technology, United Kingdom Alcoa Technical Center Argonne National Laboratory Armed Forces Radiobiology

Research Institute Army Research Laboratory

Army Research Office Auburn University

Australian Geological Survey, Australia

Australian Nuclear Science and Technology Organization, Australia Battelle Pacific Northwest Laboratory

12 Belarus Academy of Sciences, Belarus

Bethany College

Bilkent University, Turkey

Brookhaven National Laboratory

Brooklyn Polytechnic University

Brown University

California Institute of Technology

19 Carnegie Mellon University

20 Center for Advanced Research in Biotechnology

21 Centre National de Recherche Scientific, France

22 Centro Brasileiro de Pesquisas Fisicas, Brazil

23 Chalk River Laboratories, Canada

City College of New York

Clemson University

26 College of William and Mary

27 Commissariat d'Energie Atomique, France

28 Cornell University

29 Czechoslovakia Academy of Sciences, Czechoslovakia

30 Darmstadt Technology University, Germany

Dow Corning

Dow Chemical

Dupont Company

Dupont Experimental Station, Wilmington

35 Eidgenössische Technische Hochschule, Switzerland

36 Emory University School of Medicine

ExxonMobil Research and Engineering Company

Englehard Corporation

39 Federal Highway Administration

40 Florida Allantic Univrsity

Florida State University

Food and Drug Administration

43 Ford Motor Company

44 Forschungzentrum Jülich $\mathrm{GmBH}$, Germany

45 Fuji Medical Systems USA, Incorporated

46 General Electric Corporate Research

47 General Electric, Salem

48 General Motors Research and Development Center

49 Hahn-Meitner Institute, Germany

50 Hamilton College

Harvard University

52 Hamburger

Synchrotonstrahlungslabor

HASYLAB at Deutsches

Elektronen-Synchrotron DESY, Germany

53 Hongik University, South Korea
54 Hybrid Plastics

55 IBM, Almaden

56. Idaho National Engineering and Environment Laboratory

57 Imperial College, United Kingdom

58 Indiana University

59 Institut Laue-Langevin, France

60 Institut Für Physikalische Chemie, Germany

61 Institute for Energiteknikk, Norway

62 Institute of Physical and Chemical Research, Japan

63 Institute of Physics, Peoples Republic of China

64 Institute for Plasma Physics, Czech Republic

65 Institute for Reference Materials and Measurements, Belgium

66 Institute for Special Materials, Italy

67 Institute for Superhard and Novel Carbon Materials, Russia

68 Intel Corporation

69 Ioffe Physica-Technical Institute, Russia

70 John Deere Corporation

71 Johns Hopkins University

72 Joint Institute of Nuclear Research, Russia

73 Karlsruhe Nuclear Research Center, Germany

74 Kent State University

75 Kurchatov Institute, Russia

Kyoto University, Japan

Kyunghee University, Korea

Lehigh University

9 Leiden University, Germany

so Los Alamos National Laboratory

81 Los Alamos Neutron Science Center

82 Lucent Technologies, Bell Laboratories

83 Massachusetts Institute of Technology

84 Max-Planck Institut, Germany

B5 McClellan Nuclear Radiation Center

86 McMaster University, Canada

87 MDK Incorporated

88 Metal Physics Institute, Russia

89 Michigan Molecular Institute

90 Michigan State University

91 Moscow State University, Russia

92 Moscow Steel and Alloys Institute, Russia

93 National Aeronautics and Space Administration, Johnson Space Center

94 National Central University, Taiwan

95 National Institute for Research in Inorganic Materials, Japan

96 National Institute of Health

97 National Renewable Energy Laboratory

= National Sun Yat-Sen University, Taiwan

99 National Taiwan University, Taiwan

$=$ National Tsing Hua University, Taiwan

101 Naval Research Laboratory

102 Naval Surface Warfare Center

103 NEC Research Institute Incorporated

104 Neutek Incorporated

105 NIST Center for Neutron Research

106 NIST, Analytical Chemistry Division

107 NIST, Biotechnology Division

108 NIST, Building Materials Division
109 NIST, Ceramics Division

NIST, Electron and Optical Physics Division

111 NIST, lonizing Radiation Division

112 NIST, Materials Reliability Division

13 NIST, Metallurgy Division

14 NIST, Occupational Health and Safety Division

115 NIST, Polymers Division

116 NIST, Semiconductor Electronicis Division

117 NIST, Standard Reference Materials Program

118 NIST, Statistical Engineering Division

9 NIST, Surface and Microanalysis Science Division

120 NIST, Thermophysics Division

121 North Carolina State University

122 Northeastern University

123 Northern Illinois University

124 Northwestern University

125 Nova Scientific, Incorporated

126 Oak Ridge Institute for Science and Education

127 Oak Ridge National Laboratory

128 Oberlin College

129 Ohio State University

130 Ohio University

131 Olin Conservation Incorporated

132 Oregon State University

133 OSMIC, Incorporated

134 Oxford University, United Kingdom

135 Pacific Lutheran University

136 Paul Scherrer Institute, Switzerland

137 Pennsylvania State University

138 Philips Research Laboratories, The Netherlands

139 Polytechnic University

140 Princeton University

141 Purdue University

142 Queen's University, Canada

143 Racah Institute, Israel

144 Risø National Laboratory, Denmark

145 Rice University

146 Royal Institute of Technology, Sweden

147 Royal Institution, United Kingdom

148 Ruhr University, Germany

149 Russian Academy of Sciences, Russia

150 Rutgers University

151 SAGE Electrochromics

152 Sandia National Laboratory

153 Seoul National University, Korea

154 Sid Richardson Carbon Company

155 Smithsonian Institute

156 Stanford University

157 State University of New York Stony Brook

158 Stress Sensors

159 Syracuse University

160 Tata Institute of Fundamental Research, India

161 Technology for Energy Corporation

162 Technical University of Denmark Denmark

163 Technical University Munchen, Germany

164 Technobiochip

165 Tel Aviv University, Israel

166 Texas Materials Institute

167 Thomas Jefferson High School

168 Tohoku University, Japan

169 Tokyo University, Japan

170 Toyo University, Japan

171 Trinity College

172 Tufts University

173 Universita' di Brescia, Italy

174 University des Saarlandes. Germany

175 University of Aachen, Germany

176 University of Akron

177 University of Bourgogne, France

178 University of California at Berkeley

179 University of California at Davis

180 University of California at Irvine

181 University of California at Riverside

182 University of California at San Diego

183 University of California at San Francisco

184 University of California at Santa Barbara

185 University of California at Santa Cruz

186 University of Chicago

187 University of Cincinnat

188 University of Cologne, Germany

189 University of Connecticut

190 University of Delaware

191 University of Florida

192 University of Grenoble, France

193 University of Guelph, Canada

194 University of Houston

195 University of Illinois

196 University of Innsbruck, Austria

197 University of lowa

198 University of Manitoba, Canada

199 University of Maryland at College Park

200 University of Maryland Baltimore County

201 University of Massachusetts

202 University of Michigan

203 University of Minnesota

204 University of Missouri at Columbia

205 University of Missouri-Rolla

206 University of Munich, Germany

207 University of Nebraska

208 University of New Hampshire

209 University of Notre Dame

210 University of Pennsylvania

211 University of Pierre and Marie Curie, France

212 University of Puerto Rico

213 University of Rennes. France

214 University of Rhode Island

215 University of Surrey, United Kingdom

216 University of Sussex, United Kingdom 
Abdurashitov, J. N., Gavrin, V. N., Girin, S. V., Gorbachev, V. V., Ibragimova, T. V., Kalikhov, A.V., Khairnasov, N. G., Knodel, V. N., Mirmov, I. N., Shikhin, A. A., Verentenkin, E. P., Vermul, V. M., Yants, V. E., Zatsepin, G. T., Bowles, T. J., Teasdale, W. A., Wark, D. L., Cherry, M. L., Nico, J. S., Cleveland, B. T., Davis, R., Lande, K., Wildenhain, P. S., Elliott, S. R., Wilkerson, J. F., "Measurement of the Solar Neutrino Capture Rate by SAGE and Implications for Neutrino Oscillations in Vacuum," Phys. Rev. Lett. 83 (23), 4686 (1999).

Abdurashitov, J. N., Gavrin, V. N., Kalikhov, A.V., Matushko, V. L., Shikhin, A. A., Yants, V. E., Zaborskaia, O. S., Adams, J. M., Nico, J. S., Thompson, A. K., "A High Resolution Low Background Fast Neutron Spectrometer," in Proceedings of the International Workshop on Neutron Field Spectrometry in Science, Technology and Radiation Protection (Pisa, Italy, June 2000), in press.

Adams, C. P., Lee, Y. S., Lee, S. -H., Lynn, J. W., "Triple-Axis Research at NIST," Neutron News, in press

Adams, C. P., Lynn, J. W., Mukovskii, Y. M., Arsenov, A. A., Shulyatev, D. A., "Charge Ordering and Polaron Formation in the Magnetoresistive Oxide $\mathrm{La}_{0.7} \mathrm{Ca}_{0.3} \mathrm{MnO}_{3}$," Phys. Rev. Lett., in press.

Adams, J. M., "Results From the NIST Round Robin Test of Fissionable Dosimeters in a Reactor Leakage Spectrum," REACTOR DOSIMETRY, in Proceedings of the Tenth International Symposium on Reactor Dosimetry, Sept.12-17, 1999, in press.

Adams, J. M., "The Neutron Source Calibration Program at the National Institute of Standards and Technology," in Transactions of the American Nuclear Society, San Diego, CA, $82106(2000)$.

Adams, J. M., Greenhouse, L. A., "Nuclear Science at the National Institute of Standards and Technology: A Historical Overview," in Transactions of the American Nuclear Society, Winter Meeting, November 2000, (Washington, DC), in press.

Agnew, S. R., Elliott, B. R., Youngdahl, C. J., Hernker, K. J., Weertman, J. R., "Structure and Mechanical Properties of Nanocrystalline Metals-With Opportunities for Modeling." $19^{\text {th }}$ Ris $\emptyset$ International Symposium on Materials Science, Ris $\varnothing$ National Laboratory, Roskilde, Denmark, in press.

Alexandridis, P., Yang, L., "Micellization of Polyoxyalkylene Block Copolymers in Formamide" Macromol. 33 (9), 3382 (2000).

Alexandridis, P., Yang, L., "SANS Investigation of Polyether Block Copolymer Micelle Structure in Mixed Solvents of Water and Formamide, Ethanol, or Glycerol," Macromol. 33 (15), 5574 (2000).

Allman, B. E., McMahon, P. J., Nugent, K. A., Paganin, D., Jacobson, D. L., Arif, M., Werner, S. A., "Quantitative Phase Radiography with Neutrons," Nature, in press.

Amis, E. J., Bauer, B. J., Topp, A., Groehn, F., Prosa, T. J., Viers, B. D., Liu, D. W., Bames, K. A., Jackson, C. L., Nisato, G., Karim, A., Douglas, J. F., "Preparation and Characterization of Polymer/Dendrimer Blends," NISTIR 6506.

Anderson, C. R., Stirling, W. G., Andersen, K. H., Sokol, P. E., Dimeo, R. M., "TwoDimensional Excitations of Superfluid ${ }^{4} \mathrm{He}$ Confined in $72 \%$ Porosity Xerogel," Physica B 276, 820 (2000).

Anderson, D. L., Capar, S. G., "Identification of Lead in Metal Food Can Seams Using ${ }^{57}$ Co RXRFS," J. Radioanal. Nucl. Chem., in press.

Anderson, D. L., Cunningham, W. C., "Revalidation and Long-Term Stability of National Institute of Standards and Technology Standard Reference Materials 1566, 1567, 1568, and 1570," J. AOAC lnt. 83 (5), 1121 (2000).

Anderson, D. L., Cunningham, W. C., Capar, S. G., Baratta, E. J., Mackill, P., "Nuclear Methods for Food Analysis at the U. S. Food and Drug Administration," J. Radioanal. Nucl. Chem., in press.

Anderson, D.L., "Neutron Capture Prompt Gamma-Ray Activation Analysis of Meat Homogenates," J. RadioanaI. Nucl. Chem. 244 (1), 225 (2000).

Bandyopadhyay, S., Tarek, M., Klein, M. L., "Molecular Dynamics Study of a LipidDNA Complex," J. Phys. Chem. B 103, 10075 (1999).

Bandyopadhyay, S., Tarek, M., Lynch, M. L., Klein, M. L., "A Molecular Dynamics Study of the Poly (Oxyethylene) Surfactant $\mathrm{C}_{12} \mathrm{E}_{2}$ and Water," Langmuir 16 (3), 942 (1999).
Bao, W., Pagliuso, P. G., Sarrao, J. L., Thompson, J. D., Fisk, Z., Lynn, J. W., Erwin, R. W., "Incommensurate Magnetic Structure of CeRhIn ${ }_{5}$," Phys. Rev. B, in press.

Barilo, S. N., Bychkov, G. L., Kumevich, L. A.,

Barnes, J. D., Kolb, R., Barnes, K., Nakatani, A. I., Hammouda, B., "Microstructure Analysis Using Temperature-Induced Contrast Variation in Small-Angle Scattering," J. Appl. Crystallogr. 33 (1), 758 (2000).

Barnes, K. A., Karim, A., Douglas, J. F., Nakatani, A. I., Grüll, Amis, E. J., "Suppression of Dewetting in Nanoparticle Filled Polymer Films," Macromol. 33 (11), 4177 (2000).

Bateman, F. B., Boukharouba, N., Brient, C. E., Carlson, A. D., Grimes, S. M., Haight, R. C., Massey, T. N., Wasson, O. A., "New Measurements of the H(n,n)H Angular Distribution," REACTOR DOSIMETRY, in Proceedings of the Tenth International Symposium on Reactor Dosimetry, 1999, in press.

Bauer, B. J., Amis, E. J., "Characterization of Dendritically Branched Polymers by SANS, SAXS, and TEM," Tomalis/Frechet: Dendritic Polymers. 2000, in press.

Bauer, B. J., Ramzi, A., Liu, D. W., Scherrenberg, R., L., Froehling, P., Joosten, J., "Blends of Fatty Acid Modified Dendrimers With Polyolefins," J. Polym. Sci. Phys. $38(1), 95(2000)$.

Becker, D. A., "30 Years of Reactor Characterization on the NBSR," J. Radioanal. Nucl. Ch. 244 (2), 361 (2000)

Benenson, R. E., Chen-Mayer, H. H., Lynn, J. W., "Search for Effects on Neutron Transmission Due to Multiple Reflection by Glass Capillary Walls," in Proceedings of SPIE Annual Meeting, volume 3767 (1999), in press.

Blackman, M. J., Badaljan, R. S., Kikodze, Z. K., Kohl, P. L., "Chemical Characterization of Caucasian Obsidian: Geological Sources," in Synthese Sur l'Obsidienne au Proche-Orient: Du Volcan a l'Outil, (BAR Publication, London), in press.

Blackman, M. J., Redford, S., "Northern Syrian Luster and Fritware: Petrographic and Chemical Implications for Productions and Distribution," Antiquity, in press.

Bode, P., de Nadai Fernandes, E. A., Greenberg, R., "INAA as a Primary Method in Metrology for Chemical Measurements," in Proceedings Metrolsul. '99, in press.

Bode, P., de Nadai Fernandes, E. A., Greenberg, R. R., "Metrology for Chemical Measurements and the Position of INAA," J. Radioanal. Nucl. Ch. 245 (1), 109 (2000).

Bodnar, 1., Silva, A., Deitcher, R.W., Weisman, N. E., Wagner, N. J., Kim, Y. H., "Structure and Rheology of Hyper Branched Polymers and Dendritic Polymers I: Modification and Characterization of Poly(propyleneimine)-Dendrimers with Acety Groups," J. Polym. Sci. Poly. Phys. 38 (6), 857 (2000).

Bodnar, I., Silva, A., Wagner, N. J., Kim, Y. H., "Structure and Rheology of Hyperbranched Polymers and Dendritic Polymers II: Effects of Blending Acetylated and Poly(propyleneimine) Dendrimers with Aqueous Poly(ethylene oxide) Solutions," J. Polym. Sci. Poly. Phys. 38 (6), 874 (2000).

Borchers, J. A., Dura, J. A., Majkrzak, C. F., Hsu, S. Y., Lolee, R., Pratt, W. P., Bass, J., "Polarized Neutron Reflectivity Characterization of Weakly Coupled $\mathrm{Co} / \mathrm{Cu}$ Multilayers," Physica B 283 (1), 162 (2000).

Bossy, J., Anderson, K. H., Cook, J.C., Randle, O. R., "High Resolution Neutron Measurements of Roton Line Shape Under Pressure," Physica B 284, 27 (2000).

Brown, A. B. D., Rennie, A. R., "Monodisperse Colloidal Plates Under Shear" Phys. Rev E $62(1), 851(2000)$

Brown, C. M., Yildirim, T., Neumann, D. A., Heben, M. J., Gennett, T., Dillon, A. C., Alleman, J. L., Fischer, J. E., "Quantum Rotation of Hydrogen in Single-Wall Carbon Nanotubes" Chem. Phys. Lett. 329 (3), 311 (2000).

Bu, Z., Neumann, D. A., Lee, S., Brown, C. M., Engelman, D. M., Han, C. C., “A View of Dynamics Changes in the Molten Globule-Native Folding Step by Quasielastic Neutron Scattering," J. Mol. Biol. 301 (2), 525 (2000). 
Buckley, C. E., Bimbaum. H. K., Lin. J. S., Spooner, S., Bellmann, D., Staron, P., Udovic, T. J., Hollar, E., "Characterization of H-Defects in the Aluminum-Hydrogen System Using Small Angle Scattering Techniques," J. Appl. Crystallogr., in press.

Buckley, C. E., Bimbaum, H.K., Bellmann, D., Staron, P., "Calculation of the Radial Distribution Function of Bubbles in the Aluminum Hydrogen System," J. Alloy Compd. 295, 23 I (1999).

Burton, A., Feuerstein, M., Lobo, R.F. Chan, J.C.C., "Characterization of Cancrinite Synthesized in I, 3-Butanediol by Rietveld Analysis of Powder Neutron Diffraction Data and Solid-State ${ }^{23}$ Na NMR Spectroscopy," Micropor. Mesopor. Mat. 30 (2-3), 293 (1999).

Burton. A.. Lobo, R. F., "The Role of Barium Cations in the Synthesis of Low-Silica LTL Zeolites," Micropor. Mesopor. Mat. 33 (1-3), 97 (1999).

Cappelletti, R. L., "The National Institute of Standards and Technology Center for Neutron Research (NCNR)," Neutron News, in press.

Cappelletti, R. L., "Vibrational Excitations in Glasses: A: Inelastic Neutron Scattering," in Insulating and Semiconducting Glasses, Series on Directions in Condensed Matter Physics, Vol. 17, edited by P. Boolchand, chapter 6A (World Scientific Publishing Co, Singapore, 2000), p. 329.

Cappelletti, R. L., Clutter, L. K., edited by, "NIST Center for Neutron Research 1999 Programs and Accomplishments, MSEL," NISTIR 6437.

Cappelletti, R. L., Clutter, L. K., edited by, "NCNR 1999, NIST Center for Neutron Research Accomplishments and Opportunities," NIST SP 944.

Cappelletti, R. L., Glinka. C. J., Krueger, S., Lindstrom, R. A., Lynn, J. W., Prask, H., J., Prince, E., Rush. J. J., Rowe, J. M., Satija, S. K., Toby, B. H., Tsai, A., Udovic, T. J., "Materials Research With Neutrons at NIST," J. Res. Natl. Inst. Stan. Tech., in press.

Carpenter, J. M., Iverson, E. B., Mildner, D. F. R., "Time Focusing of Pulsed-Neutron Source Crystal Analyzer Spectrometers," Nucl. Instrum. Meth. A, in press.

Cava, R. J., Yamaura. K., Loureiro, S., Huang, Q., Erwin, R. W., Lynn, J. W., Young, D., "Magnets, Mischief and Metals in Cobalt Analogs of the Superconducting Cuprates," Physica $\mathrm{C}$, in press.

Chen, W. C., Heuser, B. J., King, J. S., "SANS Investigation of Deuteride (Hydride) Precipitation and Decomposition Morphology in Single Crystal Pd." J. Appl. Crystallogr., in press.

Chen, Y. Y., Lodge, T. P.. Bates, F. S., "Entropically Driven Phase Separation of Highly Branched Linear Polyolefin Blends," J. Polym. Sci. Pol. Phys., in press.

Cheng, Y. T., Mildner, D. F. R., Chen-Mayer, H. H., Sharov, V. A., Glinka, C., "An Imaging Plate Detector for Small-Angle Neutron Scattering," J. Appl. Cryst., in press.

Cheng, Y. T., Mildner, D. F. R., "A Sensitive High Resolution Neutron Imaging Detector," J. Nucl. Instrum. Meth. A, in press.

Chen-Mayer, H. H., Lamaze, G. P., Satija, S. K., "Characterization of BPSG Films Using Neutron Depth Profiling and Neutron/X-ray Reflectometry," in 2000 International Conf. on Characterization and Metrology for ULSI Technology, edited by D.G. Seiler, A.C. Diebold, W. M. Bullies, T. J. Shaffner, R. McDonald. E. J. Walters (AIP Press, Woodbury, NY), in press.

Chen-Mayer, H. H., Mackey, E. A., Paul, R. L., Mildner, D. F. R., "Quantitative Prompt Gamma Analysis Using a Focused Cold Neutron Beam," J. Radioanal. Nucl. Ch. 244 (2), 391 (2000).

Choi, S. M., Barker, J. G., Glinka, C. J., Cheng, Y. T., Gammel, P. L., "Focusing Cold Neutrons with Multiple Biconcave Lenses for Small-Angle Neutron Scattering," J. Appl. Crystallogr. 33 (1), 793 (2000).

Choi, S., Briber, R. M., Topp, A., Bauer, B. J., Tichagwa., L., Gauthier, M., "Small Angle Neutron Scattering of Arborescent Graft Polystyrenes," Macromol. 33 (17), $6495(2000)$.

Copley. J. R. D., "NIST Recommended Practice Guide; The Fundamentals of Neutron Powder Diffraction," NIST SP, in press.
Copley, J. R. D., Cook, J. C., "The Optical Filter of the Disk Chopper Spectrometer at NIST," Physica B 283 (4), 386 (2000).

Copley, J. R. D., Hayes, C., Lartigue, C., "Performance Comparisons for the ILL Neutron Spin Echo Spectrometer IN15 in its Standard and Focusing Mirror Configurations," in EUV, X-Ray and Neutron Optics and Sources, edited by C. A. MacDonald, K.A Goldberg, J. R. Maldonado, H. H. Chen-Mayer, S. P. Vernon, SPIE Symp. Series 3767 (Bellingham, Washington, 1999), p. 296

Cornell, K., Wipf, H., Cook, J. C., Kearley, G. J., Neumaier, K., "Local and Long-Range Hydrogen Diffusion in $\mathrm{NbOH}_{0.011}$," J. Alloy Compd. 295, 275 (1999).

$\mathrm{Co}_{-} \mathrm{SiO}_{2}$ Granular Films," J. Magn. Magn. Mater., in press.

Cunningham, W. C., Warner, C. R., "Br Concentration as an Indication of Pre-Baking Bromation of Bread Products," Food Addit. Contam. 17 (2), 143 (2000).

Daniel, C., Jao, T. -C., Bauer, B. J., Amis, E. J., "Study of Ethylene-Propylene Copolymer Thermoreversible Gels," Macromol. 32 (26), 8938 (1999).

Denecke, B., Eykens, R., Pauwels, J. Robouch, P. Gilliam, D. M., Hodge, P., Hutchison, J. M. R., Nico, J. S., "Characterization of Actinide Targets by Low Solid-Angle Alpha Particle Counting," Nucl. Instrum. Meth. A 438 (1), 124 (1999).

Dewey, M. S., Kessler, E. G., "Precision Measurements of Fundamental Constants Using GAMS4" J. Res. Natl. Inst. Stan. Tech. 105 (1), 11 (2000).

Dimeo, R. M., "Fourier Transform Solution to the Semi-Infinite Resistance Ladder," Am. J. Phys. 68 (669), 7 (2000).

Dimeo, R. M., Neumann, D. A., "Low Temperature Dynamics of Confined Methyl lodide," Phys. Rev B., in press.

Dimeo, R. M., Neumann, D. A., "Probing the Dynamics of Confined Systems With the NIST Backscattering Spectrometer," J. Phys. IV 10 (7), 337 (2000).

DiNoia, T. P., Kirby, C. F., van Zanten, J. H., McHugh, M. A., "SANS Study of PolymerSupercritical Fluid Solutions: Transitions From Liquid to Supercritical Fluid Solvent Quality," Macromol. 33 (17), 6321 (2000).

Dori, Y., Bianco-Peled, H., Satija, S. K., Fields, G. B., McCarthy, J. B., Tirrell, M., "Ligand Accessibility as a Means to Control Cell Response to Bioactive Bilayer Membranes," J. Biomed. Mater. Res. 50 (1), 75 (2000).

Douglas, J. F., Kent, M. S., Satija, S. K., Karim, A., "Swelling of Grafted Polymer "Brushes"," in Encyclopedia of Materials: Science and Technology, in press.

Downing, R. G., Iyengar, G. V., "Methodological Issues in the Analytical Determination of Boron," Environ. Health Perspect, in press.

Dura, J. A., Majkrzak, C. F., Krueger, S., Satija, S. K., "Subsurface Characterization by Neutron Reflectometry at NIST," in Exploration of Subsurface Phenomena by Particle Scattering, edited by N. Q. La, C. A. Melendres, S. K. Sinha (IASI Press, North East, MD, 2000), p. 245.

En, Z., Brenizer, J. S., Hostica, B., Becker, D. A., "Nitrogen Distribution Measurements by Neutron Induced Autoradiography," J. Radioanal. Nucl. Ch., in press.

En, Z., Brenizer, J. S., Hosticka, B., Becker, D. A., "Use of Nuclear Track Detection Technique to Study Boron and Nitrogen Distribution in Alloy," J. Radioanal. Nucl. Ch. 244 (2), 435 (2000).

Evmenenko, G., Bauer, B. J., Kleppinger, R., Forier, B., Dehaven, W., Amis, E. J., Miscehnko, N., Reynaers, H., "The Influence of Molecular Architecture and Solvent Type on the Size of Structure of Poly (benzyl ether) Dendrimers by SANS," Macromol., in press.

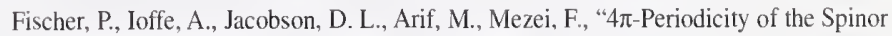
Wave Function Under Space Rotation," Nucl. Instrum. Meth. A 440 (3), 575 (2000).

Fitzsimmons, M. R., Yashar, P., Leighton, C., Schuller, I. K., Nogues, J., Majkrzak, C. F., Dura, J. A., "Asymmetric Magnetization Reversal in Exchange-Biased Hysteresis Loops," Phys. Rev. Lett. 84 (17), 3986 (2000).

Flanagan, T. B., Noh, H., Clewley, J. D., Barker, J. G., "Evidence for H-Enhanced Metal Atom Diffusion in Pd-Ni Alloys from SANS and $\mathrm{H}_{2}$ Solubilities," Scripta Mater. 39 (11), 1607 (1999). 
Fodor, J. S., Briber, R. M., Russell, T. P., Carter, K. R., Hedrick, J. L., Miller, R. D. Wong, A., "Non-Uniform Composition Profiles in Thin Film Polymeric Nanofoams," Polymer 40 (10), 2547 (1999).

Fong, H. F., Bourges, P., Sidis, Y., Regnault, L. P., Bossy, J., Ivanov, A., Milius, D. L., Aksay, 1. A., Keimer, B., "Effect of Nonmagnetic Impurities on the Magnetic Resonance Peak in $\mathrm{Yba}_{2} \mathrm{Cu}_{3} \mathrm{O}_{7}$ " Phys. Rev. Lett. 82 (9), 1939 (1999)

Fong, H. F., Bourges, P., Sidis, Y., Regnault, L. P., Bossy, J., Ivanov, A., Milius, D. L., Aksay, I. A., Keimer, B., "Spin Susceptibility in Underdoped $\mathrm{Yba}_{2} \mathrm{Cu}_{3} \mathrm{O}_{6+\mathrm{x}}$ " Phys. Rev. B 61 (21), 14773 (2000).

Foster, M. D., Greenberg, C. C., Teale, D. M., Turner, C. M., Corona-Galvan, S., Cloutet, E., Butler, P. D., Hammouda, B., Quirk, R. P., "Effective X and Surface Segregation in Blends of Star and Linear Polystyrene," in Proceedings of the Mainz Conference (1999), Macromol. Symp. 149, 263 (2000).

Freeman, K. S., Beck Tan, N. C., Trevino, S. F., Kline, S., McGown, L. B., Kiserow, D. J., "Size and Polydispersity Determinations of AOT/Bile Salt Reversed Micelles Obtained by Small-Angle Neutron Scattering," Langmuir, in press.

Gehring, P. M., Lee, S. H., Hirota, K., Masuda, T., Uchinokura, K., Hase, M., Shirane, G., "Cold Neutron Inelastic Scattering Measurements of the Spin-Peierls and Antiferromagnetic Excitations in Si-Doped $\mathrm{CuGeO}_{3}$ Single Crystals," J. Phys. Soc. Jpn. 69 (2), $592(2000)$

Gehring, P. M., Park, S. -E., Shirane, G., "Soft Phonon Anomalies in the Relaxor Ferroelectric $\mathrm{Pb}\left(\mathrm{Zn}_{1 / 3} \mathrm{Nb}_{2 / 3}\right)_{0.92} \mathrm{Ti}_{0.08} \mathrm{O}_{3}$," Phys. Rev. Lett. 84 (22), 5216 (2000)

Gehring, P. M., Vakhrushev, S. B., Shirane, G., "Soft Mode Anomalies in the Perovskite Relaxor $\mathrm{Pb}\left(\mathrm{Mg}_{\mathrm{g} / 3} \mathrm{Nb}_{2 / 3}\right) \mathrm{O}_{3}$," in Fundamental Physics of Ferroelectrics 2000 , edited by R. E. Cohen, volume 535 (American Institute of Physics, Melville, NY, 2000), p. 314 .

Geissler, E., Hecht, A. M., "Treatment of 1ncoherent Signal Subtraction" Frontiers in SAXS and SANS, ESRF-1LL-CEA Workshop (Grenoble, France, February 12-13, 1999), in press

Geissler, E., Horkay, F., Hecht, A. M., Deschamps, P., "Neutron Scattering From Elastomer Gels" Kautschuk, Gummi and Kunstoffe, in press.

Geissler, E., Horkay, F., McKenna, G. B., Deschamp, P., "Osmotic and Mechanical Observations of Swollen Polyisoprene Rubber and its Microscopic Structure" Worldwide Amazon Rubber Conference (Manus, Brazil, October 31 - November 4. 1999), in press.

Gentile, T. R., Jones, G. L., Thompson, A. K., Barker, J., Glinka, C. J., Hammouda, B., Lynn, J. W., "SANS Polarization Analysis With Nuclear-Spin-Polarized ${ }^{3} \mathrm{He}$," J. Appl. Crystallogr. 33 (1), 771 (2000)

Gentile, T. R., Jones, G. L., Thompson, A. K., Rizi, R. R., Dimitrov, I. E., Roberts, D., Gefter, W., Schnall, M. D., Leigh, J. S., "A Compact Compressor for Application of Metastability-Exchange Optical Pumping of ${ }^{3} \mathrm{He}$ to Human Lung Imaging," Magn. Reson. Med., in press.

Gentile, T. R., Jones, G. L., Thompson, A. K., Rizi, R. R., Dimitrov, I. E., Roberts, D., Gefter, W., Schnall, M. D., Leigh, J. S., "Demonstration of a Compact Compressor for Application of Metastability-Exchange Optical Pumping of ${ }^{3} \mathrm{He}$ to Human Lung Imaging," Magnet. Reson. Med., 43 (2), 290 (2000).

Gentile, T. R., Rich, D. R., Thompson, A. K., Bailey, C., Gericke, M., Hussey, D., Neff, B., Snow, W. M., Jones, G. L., Wildman, E., "The N1ST-Indiana-Hamilton Polarized ${ }^{3}$ He Spin Filter Program," in Proceedings Fundamental Plyysics with Pulsed Neutron Beams Conference, TUNL, Durham, NC, June 1-2, 2000, (World Scientific Inc.), in press.

Gibbons, M. R., Richards, W. J., Shields, K., Paul, R. L., Lindstrom, R. M., "Correction of Scattering and Beam Hardening in Thermal Neutron Topography for Quantitative Detection of Hydrogen," IEEE Trans. Nucl. Sci., in press.

Giebultowicz, T. M., Kepa, H., Blinowski, J., Kacman, P., "Neutron Scattering Studies of Interlayer Correlations in magnetic Semiconductor Superlattices," Physics E, in press.

Gilliam, D. M., "NIST Neutron Physics and Dosimetry," in Transactions of the American Nuclear Society/European Nuclear Society Winter Meeting, November 12-I6, 2000, (Washington, DC.), in press.

Glinka, C. J., "Sixth Annual Summer School Held at NIST," Neutron News 11 (4), 12 (2000).

Gnäupel-Herold, T., Brand, P. C., Prask, H. J., "Accessing the Elastic Properties of Cubic Materials with Diffraction Methods," Advances in X-ray Analysis 42, 464 (1998).
Gnäupel-Herold, T., Haeffner, D. R., Prask, H. J., Matejicek, J. "Stress Determination by Whole Pattern Analysis-Application to Synchrotron HEXRD Measurements on Thermal Sprayed Coatings," in The Sixth International Conference on Residual Stresses, (1OM, London), in press.

Gnäupel-Herold, T., Matejicek, J., Prask, H. J., "Mechanical Properties of Plasma Sprayed Coatings - Measured by Diffraction," in $9^{\text {th }}$ International Metallurgical Conference Metal 2000, in press.

Gnäupel-Herold, T., Prask, H. J., "The Calculation of Diffraction Elastic Constants for Arbitrary Aggregates," Phys. Rev. B, in press.

Gnäupel-Herold, T., Prask, H. J., "Diffraction Elastic Constants for Arbitrary Specimen and Crystal Symmetries: Theory and Practical Consequences," in The Sixth International Conference on Residual Stresses (IOM, London), p. 243.

Gnäupel-Herold, T., Prask. H. J., Clark, A. V., Hehman, C. S., Nguyen. T. N., "A Comparison of Neutron and Ultrasonic Determinations of Residual Stress," Meas Sci. Technol. 11 (4), 436 (2000).

Gnäupel-Herold, T., Prask. H. J., Magiera, J., "Effect of Grinding Strategy on Accumulation of Damage in Rails: Neutron Diffraction Investigation of Residual Stresses in Transverse and Oblique Cut Rail Slices," in The Sixth International Conference on Residual Stresses (IOM, London), in press.

Green, M. A., Neumann, D. A., "Synthesis, Structure and Electronic Properties of $\mathrm{LaCa}_{2} \mathrm{Mn}_{2} \mathrm{O}_{7}$," Chem. Mater. 12 (1), 90 (2000).

Green, M. A., Prassides, K., Day, P., Neumann, D. A., "Structure of the $n=2$ and $n=$ Member for the Ruddlesden-Popper Series, $\mathrm{Sr}_{n+1} \mathrm{Sn}_{n} \mathrm{O}_{3 n+1}$ "' Int. J. Inorg. Mater. 2 (1), 35 (2000).

Greenberg, C. C., Foster, M. D., Turner, C. M., Corona-Galvan, S., Cloutet, E., Butler, P. D., Hammouda, B., Quirk, R. P., "Effective Interaction Parameter Between Topologically Distinct Polymers," Polymer 40 (16), 4713 (1999).

Greenberg, C. C., Teale, D. M., Foster, M. D., Turner, C. M., Corona-Galvan, S., Cloutet, E., Quirk, R. P., Majkrzak, C. F., Demaree, D., "Surface Segregation in Blends of Linear and Regularly Star Branched Polystyrene," Macromol., in press.

Greenberg, R. R., Lindstrom, R. M., Simons, D. S., "Instrumental Neutron Activation Analysis for Certification of Ion-Implanted Arsenic in Silicon," J. Radioanal. Nucl. Ch. 245 (1), 57 (2000)

Grohn, F., Bauer, B. J., Akpalu, Y. A., Jackson, C. L., Amis, E. J., "Dendrimers as Templates for the Formation of 1norganic Nanoclusters," Macromol., in press.

Grohn, F., Bauer, B. J., Akpalu, Y. A., Jackson, C. L., Amis, E. J., "Dendrimer Templates for the Formation of Gold Nanoclusters," Macromol. 33 (16), 6042 (2000).

Grohn, F., Bauer, B. J., Amis, E. J., "Organic-Inorganic Hybrid Particles by Dendrimer Nanotemplating," in Proceedings of the Materials Research Society Meeting (April 2000), in press.

Gruell, H., Schreyer, A., Berk, N. F., Majkrzak, C. F., Han, C. C., "Composition Profiling in a Binary Polymer Blend Thin Film Using Polarized Neutron Reflectivity," Europhys. Lett. 50 (I), 107 (2000).

Gruell, H., Sung, L., Karim, A., Douglas, J. F., Satija, S. K., Hayashi, M., Jinnai, H., Hashimoto, T., Han, C. C., "Surface Segregation of a Symmetrical Segregating Polymer Blend Film Above and Below the Critical Point of Phase Separation," Phys. Rev. Lett., in press.

Grundl, J. A., "NIST Cavity Fission Source (CFS) - Facility Characteristics," NISTIR 6420 .

Grundl, J. A., "NIST Cavity Fission Source - Operations Manual," NISTIR 6419

Gygax, F. N., Solt, G., Amato, A., Anderson, I. S., Pinkpank, M., Schenk, A., Udovic T. J., "Light Hydrogen Isotope + in $\mathrm{Sc}$ and $-\mathrm{ScH}_{\mathrm{x}}$ Solid Solutions," Phys. Rev. B $61(1), 168(2000)$

Hagemann, 1. S., Huang, Q., Gao, X. P. A., Ramirez, A. P., Cava, R. J., "Geometric Magnetic Frustration in $\mathrm{Ba}_{2} \mathrm{Sn}_{2} \mathrm{ZnGa}_{4} \mathrm{Cr}_{6} \mathrm{O}_{22}$ : a Two-Dimensional Spinel Based Kagome Lattice," Phys. Rev. Lett., in press.

Hammouda, B., "SANS From Polymers Under Pressure," Neutron News, in press.

Hawari, A. I., Adams, J. M., "A Method for the Minimization of the HPGe Detector Efficiency Bias in the Measurement of the Spectral Index," REACTOR DOSIMETRY, in Proceedings of the Tenth 1nternational Symposium on Reactor Dosimetry, Sept. 12-17, 1999, ASTM STP 1398, in press.

Hayashi, M.. GrueIl, H., Esker, A. Weber M., Sung, L., Satija, S. K., Han, C. C., Hashimoto, T. A, "Neutron Reflectivity Study of Diblock Formation During Reactive Blending Processes," Macromol. 33 (17), 6485 (2000). 
Hayashi, M., Hashimoto, T., Hasegawa, H., Takenaka, M.. Grüll, H., Esker, A. R., Weber, M., Satija, S. K., Han, C. C., Nagao, M., "Interface Between a Polysulfone and Polyamide as Studied by Combined Neutron Reflectivity and Small-Angle Neutron Scattering Techniques," Macromol., in press.

Hayashi, M., Hashimoto, T., Weber, M., Gruell, H., Esker, A., Han, C. C., Satija, S. K., "Transient Interface Instability in Bilayer Polymer Films as Observed by Neutron Reflectivity Studies," MacromoI., in press.

He, T., Huang, Q., Cava, R. J., "Comparison of the Magnetic Properties of Isoelectronic $\mathrm{Sr}_{\mathbf{x}}\left(\mathrm{Na}_{0.5} \mathrm{La}_{0.5}\right)_{1-x} \mathrm{RuO}_{3}$ and $\mathrm{Sr}_{x} \mathrm{Ca}_{1-\mathrm{x}} \mathrm{RuO}_{3}$," Phys. Rev. B, in press.

Heath, C. H., Streletzky, K. A., Woelk, J. Wyslouzil, B. E., Strey, R., "D $\mathrm{D}_{2} \mathrm{O}-\mathrm{H}_{2} \mathrm{O}$ Condensation in Supersonic Nozzles I Experiments." in Nucleation and Atmospheric Aerosols, edited by B. Hale, M. Kulmala (American Institute of Physics, 2000), p. 59.

Heath, C. H., Streletzky, K. A., Woelk, J. Wyslouzil, B. E., Strey, R., "D, $\mathrm{D}_{2} \mathrm{H}_{2} \mathrm{O}$ Condensation in Supersonic Nozzles II Modeling Experiments," in Nucleation and Atmospheric Aerosols, edited by B. Hale, M. Kulmala (American Institute of Physics, 2000), p. 63.

Heene, M. M., Wipf, H., Udovic. T. J., Rush. J. J., "Hydrogen Vibrations and Hydrogen Trapping in $\mathrm{TaN}_{0.006} \mathrm{H}_{0.003}$, J. Phys. Condens. Mat. 12 (28), 6183 (2000).

Heller-Zeisler, Borgoul, P. V., Moore, R. R., Smoliar, M., Suarez, A. E., Ondov, J. M., "Comparison of INAA and RNAA Methods With Thermal-Ionization Mass Spectrometry for Iridium Determinations in Atmospheric Tracer Studies," J. Radioanal. Nucl. Chem. 244 (1), 93 (2000).

Heller-Zeisler, S. F., Ondov, J. M., Zeisler, R., "Collection and Characterization of a Bulk PM2.5 Air Particulate Matter Material for use in Reference Materials," Biol. Trace Elem. Res. 71 (2), 195 (1999).

Herwig, K. W., Fuhrmann, D., Criswell, L., Taub, H., Hansen, F. Y., Dimeo, R., Neumann, D. A., "Dynamics of Intermediate-Length Alkane Films Absorbed on Graphite," J. Phys. IV 10 (7), 157 (2000).

Heuser B. J., Chen, W. C., " Comparison of Deutride (Hydride) Morphology During Precipitation and Decomposition in Single Crystal Pd: A Clue to the Pressure Hysteresis," Phys. Rev. B, in press.

Hillmyer, M. A., Maurer, W. W., Lodge, T. P., Bates, F. S., AlmdaI, K., "Model Bicontinuous Microemulsions in Ternary Homopolymer/Block Copolymer Blends," J. Phys. Chem. B 103 (23), 4814 (1999)

Hone, J. H. E., Howe, A. M., Cosgrove, T., "A Rheological and Small Angle Neutron Scattering Study of the Structure of Gelatin/Polyelectrolyte Complexes Under Shear" Macromol., in press.

Hone, J. H. E., Howe, A. M., Cosgrove, T., "A Small Angle Neutron Scattering Study of the Structure of Gelatin/polyelectrolyte Complexes," Macromol. 33 (4), 106 (2000).

Horkay, F., Basser, P. J., Hecht, A. M., Geissler, E., "Osmotic and SANS Observations on Sodium Polyacrylate Hydrogels in Physiological Salt Solutions," Macromol., in press.

Horkay, F., Hecht, A. M., Geissler, E., "Neutron Scattering Investigations on Bimodal Polymer Gels" J. Polym. Sci., Polym. Phys. Ed., in press.

Horkay, F., Hecht, A. M., Geissler, E., "Neutron Scattering Investigations on Filled Polymer Gels" in Proceedings 219 th ACS National Meeting 41 (San Francisco, CA, March 26-30, 2000), in press.

Horkay, F., Hecht, A. M., Geissler, E., "Neutron Scattering Observations on Filled Poly (dimethysiloxane) Gels," in Proceedings $218^{\text {th }}$ ACS National Meeting, Division of Colloid \& Surface Chemistry 68 (New Orleans, August 22-26, 1999), in press.

Horkay, F., McKenna, G. B., Deschamps, P.. Geissler, E., "Neutron Scattering Properties of Randomly Cross-Linked Polyisoprene Gels," Macromol., in press.

Horkay, F., McKenna, G. B., Deschamps, P., Geissler, E., "Relationship Between Microscopic Structure and Osmotic and Mechanical Behavior of Polysoprene Gels," Macromol., in press.

Horkay, F., McKenna, G. B., Geissler, E., "Nanostructures in Swollen Polymer Networks" in Proceedings Polymer Networks 98, 14 ${ }^{\text {h }}$ Polymer Networks Group Conference (Trondheim, Norway, June 28-July I, 1998), in press.

Huang, E., Mansky, P., Russell, T. P., Harrison, C., Chaikin, P. M., Register, R. A., Hawker, C. J., Mays, J., "Mixed Lamellar Films: Evolution, Commensurability Effects, and Preferential Defect Formation," Macromol. 33 (1), 80 (2000).
Huang, Q., Lynn, J. W., Erwin, R. W., Santoro, A., Dender, D. C., Smolyaninova, V. N., Ghosh, K., Greene, R. L., "Temperature and Field Dependence of the Phase Separation, Structure, and Magnetic Ordering in $\mathrm{La}_{1-x} \mathrm{Ca}_{\mathrm{x}} \mathrm{MnO}_{3},(\mathrm{x}=0.47,0.50$, and 0.53)," Phys. Rev. B 61 (13), 8895 (2000).

Huffman, P. R., Brome, C. R., Butterworth, J. S., Coakley, K. J., Dewey, M. S., Dzhosyuk, S.N., Golub, R., Greene, G. L., Habicht, K., Lamoreaux, S. K., Mattoni, C. E. H., McKinsey, D. N., Wietfeldt, F. E., Doyle, J. M., "Magnetic Trapping of Neutrons," Nature 403 (6765), 62 (2000).

Huffman, P. R., Brome, C. R., Butterworth, J. S., Coakley, K. J., Dewey, M. S., Dzhosyuk, S. N., Gilliam, D. M., Golub, R., Greene, G. L., Habicht, K., Jones, G. L., Lamoreaux, K. S., Mattoni, C. E., McKinsey, D. N., Wietfeldt, F. E., Doyle, J. M., "Progress Towards Magnetic Trapping of Ultracold Neutrons," Nucl. Instrum. Meth. A 440 (3), $522(2000)$

Huffman, P. R., Brome, C. R., Butterworth, J. S., Dzhosyuk, S. N., Golub, R., Lamoreaux, S. K., Mattoni, C. E. H., McKinsey, D. N., Doyle, J. M., "Magnetically Stabilized Luminescent Excitations in Hexagonal Boron Nitride," Journal of Luminescence, in press.

Huffman, P. R., Coakley, K. J., Thompson, A. K., Yang, G. L., Wietfeldt, F. E., Alvine, K. J., Brome, C. R., Dzhosyuk, S. N., Mattoni, C. E. H., Michniak, R. A., McKinsey, D. N., Yang, L., Doyle, J. M., Golub, R., Habicht, K., Koch, J., Lamoreaux, S. K., Leite, M. L., Maia, L., Rodrigues, A. C., Zanotto, E. D., "Magnetic Trapping of Ultracold Neutrons: Prospects for an Improved Measurement of the Neutron Lifetime," in Conference Proceedings for the Workshop on Fundamental Physics with Pulsed Neutron Beams (World Scientific Inc.), in press.

Hutson, N. D., Reisner, B. A., Yang, R. T., Toby, B., "Silver Ion-Exchanged Zeolites Y, $X$, and Low Silica X: Observations of Thermally Induced Cation/Cluster Migration and the Resulting Effects on the Equilibrium Adsorption of Nitrogen," Chem. Mater. 12 (10), $3020(2000)$.

Hwang, S. -R., Li, W. -H., Lee, K. C., Lynn, J. W., Wu, C. -G.. "Spiral Magnetic Structure of Fe in Van Der Waals Gaped FeOCI," Phys. Rev. B, in press.

Iampietro, D. J., Kaler, E. W., "Phase Behavior and Microstructure of Aqueous Mixtures of Cetyltrimethylammonium Bromide and Sodium Perfluorohexanoate," Langmuir 15 (25), $8590(2000)$.

Ichikawa, N., Uchida, S., Tranquada, J. M., Niemöller, T., Gehring, P. M., Lee, S. -H., Schneider, J. R., "Local Magnetic Order vs. Superconductivity in a Layered Cuprate," Phys. Rev. Lett. 85 (8), 1738 (2000).

Ilavsky, J., Long, G. G., Allen, A. J., Berndt, C. C., "Evolution of the Void Structure in Plasma-Sprayed YSZ Deposits During Heating," Mat. Sci. Eng. A-Struct. 272 (1), 215 (1999).

Ilavsky, J., Stalick, J. K., "Phase Composition and Its Changes During Annealing of Plasma-Sprayed YSZ," Surf. Coat. Tech. 127 (2), 120 (2000).

Ilavsky, J., Stalick, J. K., Wallace, J., "Thermal-Spray Yttria-Stabilized Zirconia Phase Changes During Annealing," in Proceedings of the International Thermal Spray Conference, May 2000, in press.

Ioffe, A., Arif, M., Jacobson, D. L., Mezei, F., "Comment on "Precision Neutron Interferrometric Search for Evidence of Nuclear Quantum Entanglement in Liquid $\mathrm{H}_{2} \mathrm{O}-\mathrm{D}_{2} \mathrm{O}$ Mixtures" Ioffe et al. Reply," Phys. Rev. Lett. 84 (9), 2037 (2000).

Ioffe, A., Arif, M., Jacobson, D., Mezei, F., "Does Quantum Entanglement Invalidate Contrast Variation Practices in Neutron Scattering?," Physica B 276, 952 (2000).

Ioffe, A., Vrana, M., Arif, M., Jacobson, D., "The Study of the Electrical Charge Distribution Inside the Neutron by Neutron Scattering Methods," Physica B 276, 968 (2000).

Ivkov, R., Gehring, P. M., Maliszewskyj, N. C., Krishnamoorti, R., "Structure and Dynamics of Polymers in Confined Geometries: Neutron Studies of Intercalated Clay-Polymer Nanocomposites," Proc. Soc. Plastics Engineers-ANTEC' '99, in press.

Ivkov, R., Papanek, P., Gehring, P. M., Krishnamoorti, R., "Dynamics of Confined Polymers Measured by Inelastic Neutron Scattering," Polym. Mat. Sci. Eng. Prep. $82,210(2000)$.

lzumi, F., Ikeda, T., "A Rietveld-Analysis Program RIETAN-98 and Its Applications to Zeolites," Mater. Sci. Forum 321 (3), 198 (2000).

Jacobson, D. L., Arif, M., Bergmann, L., Ioffe, A., "Development of the Neutron Phase Contrast Imaging Technique and its Application in Materials Science Research." in Proceedings, SPIE conference, Denver, July 17-23, 1999, in press. 
James, W. D., Zeisler, R., "Uptake of Oxygen in a Coal Standard Reference Material Determined by $14-\mathrm{MeV}$ Neutron Activation Analysis," J. Radioanal. Nucl. Ch., in press.

Jones, G. L., Adams, J. M., Anaya, J. M., Bowles, T. J., Chupp, T. E., Coulter, K. P., Dewey, M. S., Freedman, S. J., Fujikawa, B. K., Garcia, A., Greene, G. L., Hwang, S. -R., Lising, L. J., Nico, J. S., Robertson, R. G. H., Steiger, T. D., Teasdale, W. A., Thompson, A. K., Wietfeldt, F. E., and Wilkerson, J. F., "Time Reversal in Polarized Neutron Decay - The EMIT Experiment," Nucl. Instrum. Meth. A 440 (3), $648(2000)$.

Jones, G. L., Gentile, T. R., Thompson, A. K., Chowdhuri, Z., Dewey, M. S., Snow, W. M., Wietfeldt, F. E., "Test of 'He-Based Neutron Polarizers at NIST," Nucl. Instrum. Meth. 440 (3), 772 (2000).

Jones, R. L., Kumar, S. K., Ho, D. L., Briber, R. M., Russell, T. P., "Chain Conformation in Ultrathin Polymer Films Using Small Angle Neutron Scattering," Macromol., in press

Kamitakahara, W. A., Wada, N., "Dynamics of Intercalated Water Molecules in Synthetic Layered Silicates," Mol. Cryst. Liq. Cryst. 341, 1.307 (2000).

Karen, P., Kjekshus, A., Huang, Q., Karen, V., Mighell, A. D., Santoro, A., "Neutron Powder Diffraction Study of the Crystal Structure of the Oxycarbonate Phase $\mathrm{YBa}_{2} \mathrm{Cu}_{2.85}\left(\mathrm{CO}_{3}\right)_{0.15} \mathrm{O}_{6.73}$," Physica C 336 (3), 279 (2000).

Keimer, B., Casa. D., Ivanov, A., Lynn, J. W., Zimmermann, M. V., Hill, J. P., Gibbs, D., Taguchi, Y., Tokura, Y., "Spin Dynamics and Orbital State in $\mathrm{LaTiO}_{3}$," Phys. Rev Lett., in press.

Kent, M., "A Quantitative Study of Tethered Chains in Various Solution Conditions Using Langmuir Diblock Copolymer Monolayers," Macromol., in press.

Kepa, H., Kutner-Pielaszek, J., Twardowski, A., Sipatov, A. Y., Majkrzak, C. F., Story, T., Galazka, R. R., Giebultowicz, T. M., "Interlayer Correlations in Ferromagnetic Semiconductor Superlattices EuS/PbS," J. Magn. Magn. Mater., in press.

Khalifah, P., Huang, Q., Lynn, J. W., Erwin, R. W., Cava, R. J., "Synthesis and Crystal Structure of $\mathrm{La}_{3} \mathrm{RuO}_{7}$ " Mater. Res. Bull. 35 (1), 1 (2000).

Kilic, C., Ciraci, S., Gulseren, O., Yildirim, T., "Variable and Reversible Quantum Structures on a Single Carbon Nanotube," Phys. Rev. B, in press.

Kilic, C., Yildirim, T., Mehrez, H., Ciraci, S., "A First-Principles Study of the Structure and Dynamics of $\mathrm{C}_{8} \mathrm{H}_{8} \mathrm{Si}_{8} \mathrm{H}_{8}$ and $\mathrm{Ge}_{8} \mathrm{H}_{8}$ Molecules," J. Phys. Chem. A 104 (12), $2724(2000)$.

Kim, S. D., Klein, A., Sperling, L. H., Boczar E.M., Bauer, B. J., "A Direct Comparison of SANS and DET for Polymer Diffusion in Polystyrene Latex Film," Macromol., in press.

Kim, S. D., Klein, A., Sperling, L. H., Boczar, E. M., Bauer, B. J., "SANS Study of Sulfite End Group Effect on Polystyrene Self-Diffusion," Macromol., in press.

King, H. E., Hutter, J. L., Lin, M. Y., Sun, T., "Polymer Conformations of Gas-Hydrate Kinetic Inhibitors: A Small-Angle Neutron Scattering Study," J. Chem. Phys. 112 (5), $2523(2000)$.

Kline, S. R., "Structural Evolution During Micelle Polymerization," J. Appl. Crystallogr. $33(1), 618(2000)$.

Kmety, C. R., Huang, Q., Lynn, J. W., Erwin, R. W., Manson, J. L., McCall, S., Crow, J. E., Stevenson, K. L.. Miller, J. S., Epstein, A. J., "Noncollinear Antiferromagnetic Structure of the Molecule-Based Magnet Mn[N(CN) $]_{2}$," Phys. Rev. B 62 (9), 5576 (2000).

Kmety, C. R., Manson, J. L., Huang, Q., Lynn, J. W., Erwin, R. W., Miller, J. S., Epstein, A. J., "Magnetic Phase Transitions in $\mathrm{M} "\left[\mathrm{~N}(\mathrm{CN})_{2}\right]_{2}$ " Mol. Cryst. Liq. Cryst. A 334, $631(1999)$

Kobayashi, K., Huang, C. l., Lodge, T. P., "Thermoreversible Gelation of Aqueous Methylcellulose Solutions," Macromol. 32 (21), 7070 (1999).

Koehler, R. D., Raghavan, S. R., Kaler, E. W., "Microstructure and Dynamics of Wormlike Micellar Solutions Formed by Mixing Cationic and Anionic Surfactants," J. Phys. Chem. B, in press.

Kolb, R., Lin, E. K., Wu, W. L., Satija, S. K., "Neutron Reflectivity Measurements of Polymer Melt Mobility Near Oxidized Silicon Surfaces Modified with SelfAssembled Monolayers," Langmuir (in press).

Koubi, L., Tarek, M., Klein, M. L., Scharf, D., "Distribution of Halothane in a Dipalmitoylphosphatidylcholine Bilayer from Molecular Dynamics Calculations," Biophys. J. 78 (2), 800 (2000)
Krishnamoorti, R., Silva, A. S., Modi, M. A., Hammouda, B., "Small Angle Neutron Scattering Study of a Cylinder to Sphere Order-Order Transition in Block Copolymers," Macromol. 33 (10), 3803 (2000).

Krone, C. A., Robisch, P. A., Tilbury, K. L., Stein, J. E., Mackey, E. A., Becker, P. R., O'Hara, T. M., Philo, L. M., "Heavy Metals and Other Elements in Liver Tissues of Bowhead (Balaena Mysticetus) Whales," Mar. Mam. Sci., in press.

Krueger, J. K., Gallagher, S. C., Wang, C. L., Trewhella, J., "Calmodulin Remains Extended Upon Binding to Smooth Muscle Caldesmon: A Combined Scattering and Fourier Transform Infrared Spectroscopy Study" Biochem., in press.

Krueger, J. K., Trewhella, J., "Neutron Scattering Studies of Muscle Protein Structures and Their Interactions" in Molecular Interactions of ActinL Actin Structure and ActinBinding Proteins, edited by C. G. do Remedios, D. D. Thomas, (Springer Verlag, 1999), in press.

Krueger, S., Gregurick, S., Shi, Y., Wang, S., Wladkowski, B. D., Schwarz, F. P., "Specific DNA Binding to CRP Induces a Conformational Change in CRP in Solution: A Small Angle Neutron Scattering Study," Biochem., in press.

Krueger, S., Meuse, C. W., Majkrzak, C. F., Dura, J. A., Berk, N. F., Tarek, M., Plant, A. L., "Investigation of Hybrid Bilayer Membranes With Neutron Reflectometry: Probing the Location of lnteractions of Melittin," Langmuir, in press.

Lamaze, G. P., Chen-Mayer, H. H., "Nitrogen Analysis in Thin Films Using Cold Neutron Depth Profiling," in Proceedings $9^{\text {th }}$ Intl. Conf. on Modern Materials and Technologies, edited by P. Vincenzini, (TECHNA, Faenza, ltaly), in press.

Lamaze, G. P., Chen-Mayer, H. H., Badding, M., Laby, L., "In Situ Measurement of Lithium Movement in Thin Film Electrochromic Coatings Using Cold Neutron Depth Profiling," Surf. Interface Anal. 27 (7), 644 (1999).

Lamaze, G. P., Chen-Mayer, H. H., Gerouki, A., Goldner, R. B., "Analysis of Lithium Transport in Electrochromic Multilayer Films by Neutron Depth Profiling," Surf. Interface Anal. 29 (9), 638 (2000)

Lamaze, G. P., Chen-Mayer, H. H., Langland, J. K., "Recent Developments in Neutron Depth Profiling at NIST," in 1998 International Conference on Characterization Metrology for Ultra Large Scale Integration Technology, edited by D. G. Seiler, A. C. Diebold, W. M. Bullis, T. J. Shaffner, R. McDonald, E. J. Walters, (AIP, Woodbury, NY, 1998), p. 883

Lee, A. S., Gast, A. P., Butun, V., Armes, S. P., "Characterizing the Structure of pH Dependent Polyelectrolyte Block Copolymer Micelles" Macromol. 32 (13), 4302 (1999).

Lee, S. -H., Cheong, S. -W., Yamada, K., Majkrzak, C. F., "Charge and Canted Spin Order in $\mathrm{La}_{2 . x} \mathrm{Sr}_{x} \mathrm{NiO}_{4}$," Phys. Rev. B, in press.

Lee, S. -H., Majkrzak, C. F., Sinha, S. K., Stassis, C., Kawano, H., Lander, G. H., Brown, P. J., Fong, H. F., Cheong, S. W., Matsushita, H., Yamada, K., Endoh, Y., "Search for Orbital Moments in Underdoped Cuprate Metals," Phys. Rev. B 60 (14), 10405 (1999).

Lee, S. -H., Broholm, C., Kim, T. H., Ratcliff II, W., Cheong, S.-W. "Local Spin Resonance and Spin-Peierls Like Phase Transition in the Geometrically Frustrated Antiferromagnet," Phys. Rev. Lett 84 (16), 3718 (2000).

Lee, Y. S., Birgeneau, R. J., Kastner, M. A., Endoh, Y., Wakimoto, S., Yamada, K., Erwin, R. W., Lee, S. -H., Shirane, G., "Neutron-Scattering Study of Spin-Density Wave Order in the Superconducting State of Excess-Oxygen-doped $\mathrm{La}_{2} \mathrm{CuO}_{4+y}$, Phys. Rev. B 60 (5), 3643 (1999)

Lefebvre, A. A., Lee, J. H. Balsara. N. P., Hammouda, B., "Neutron Scattering From Pressurized Polyolefin Blends Near the Limits of Metastability," Macromol., in press.

Lefebvre, A. A., Lee, J. H., Balsara, N. P., Hammouda. B., "Fluctuations in Highly Metastable Polymer Blends," J. Polym. Sci. Pol. Phys. 38 (14), 1926 (2000).

Li, W. -H., Wu, S. Y., Lee, K. C., Lynn, J. W., "Magnetic Ordering of Mn in Ca-doped $\mathrm{NdMnO}_{3}$," Physica B 276, 724 (2000).

Liao, C. Y., Choi, S.M., Mallamace, F., Chen, S. H., "SANS Study of the Structure and Interaction of L64 Triblock Copolymer Micellar Solution in the Critical Region," J. Appl. Crystallgr. 33 (1), 677 (2000).

Licci, F., Marezio, M., Huang, Q., Santoro, A., Bougerol-Chaillout, C., Masini, R., "Synthesis, Structure and Superconductivity of $\mathrm{Hg}_{0.75} \mathrm{Mo}_{0.25} \mathrm{Ba}_{2} \mathrm{CuO}_{4+}$," Physica C 325 (1), 41 (1999).

Likos, C. N., Vaynberg, A., Lowen, H., Wagner, N. J., "Colloidal Stabilization by Adsorbed Gelatin" Langmuir, in press. 
Lin. E. K.. Kolb, R., Satija, S. K., Wu. W. L., "Enhanced Polymer Segment Exchange Kinetics due to an Applied Shear Field," Macromol. 32 (14), 4741 (1999).

Lin, E. K., Kolb. R., Satija, S. K., Wu, W. L., "Reduced Polymer Mobility Near the Polymer/Solid Interface as Measured by Neutron Reflectivity," Macromol. 32 (11), 3753 (1999)

Lin. E. K., Lee. H. J., Wang, H., Wu. W. L.. "Structure and Property Characterization of Low-k Dielectric Porous Thin Films Determined by X-Ray Reflectivity and SmallAngle Neutron Scattering," in $2000 \mathrm{Intl}$. Conf. on Characterization and Metrology for ULSI Technology: (July 2000), in press.

Lin. E. K., Pochan, D. J., Wu, W. L.. Satija, S. K., "Neutron Reflectivity Measurements of Molecular Mass Effects on Polymer Mobility Near the Polymer/Solid Interface," in Proceedings of the Materials Research Society Meeting (April 2000), in press.

Lin. E. K., Wu, W. L.. Jin, C., Wetzel, J. T., "Structure and Property Characterization of Porous Low-k Dielectric Constant Thin Films Using X-ray Reflectivity and SmallAngle Neutron Scattering," in Proceedings of the Materials Research Society Meeting (April 2000), in press.

Ling, X. S., Park, S. R., Choi, S. M., Dender, D. C., Lynn, J. W., "Direct Observation of Superheating and Supercooling through the First-Order Vortex Solid-Liquid Transition," Phys. Rev. Lett., in press.

Lising, L. J., Hwang. S. R., Adams, J. M., Bowles, T. J., Browne, M. C., Chupp, T. E., Coulter, K. P., Dewey, M. S., Freedman, S. J., Fujikawa, B. K., Garcia, A., Greene, G. L., Jones, G. L., Mumm. H. P., Nico, J. S., Richardson, J. M., Robertson, R. G. H. Steiger, T. D., Teasdale, W. A., Thompson, A. K., Wasserman, E. G., Wietfeldt, F. E., Welsh. R. C., Wilkerson, J. F., "New Limit on the $D$ Coefficient in Polarized Neutron Decay," Physical Review C 62055501 (2000).

Liu. W. J., Jeng. U., Lin, T. L.. Lai, S. H., Shih, M. C., Tsao, C. S., Chiang, L. Y., Sung, L. P., "Adsorption of Dodecahydroxylated-Fullerene Monolayers at the Air-Water Interface", Physica B, in press.

Livingston. R., Neumann, D. A., Allen, A. J., FitzGerald, S. A., Berliner, R., "Application of Neutron Scattering to Portland Cement," Neutron News, in press.

Lobry, L., Micali, N., Mallamace, F.. Liao, C., Chen, S. H., "Interaction and Percolation on L6+ Triblock Copolymer Micellar System," Phys. Rev. E 60 (6), 7076 (1999).

LoNostro. P., Choi S. -M., Ku, C. -Y., Chen, S. H., "Fluorinated Microemulsions: A Study of the Phase Behavior and Structure," J. Phys. Chem. B 103 (25), 5341 (1999)

Loureiro, S. M., Felser, C., Huang, Q., Cava, R. J., "Refinement of the Crystal Structures of Strontium Cobalt Oxychlorides by Neutron Powder Diffraction," Chem. Mater., in press.

Lynn, J. W., "Magnetic Neutron Scattering," in Methods in Materials Research, edited by Kaufman (John Wiley \& Sons), in press.

Lynn, J. W., "Magnetic Structure Determination at NBS/NIST," ACA Newsletter, in press.

Lynn, J. W., "Neutron Studies of Exchange-Coupled Superconductors," in Proceedings NATO Advanced Research Workshop (June 13-18 $8^{\text {th }}$, in press.

Lynn. J. W., "Progress in Borocarbide Research," in Proceedings NATO Conference, in press.

Lynn. J. W., Keimer, B., Ulrich, C., Bernhardt, C., Tallon, J. L., "Antiferromagnetic Ordering of $\mathrm{Ru}$ and $\mathrm{Gd}$ in Superconducting $\mathrm{RuSr}_{2} \mathrm{GdCu}_{2} \mathrm{O}_{8}$," Phys. Rev. B 61 (22), R14 964 (2000).

Lynn. J. W., Rosov, N., Barilo. S. N., Kurnevitch. L., Zhokhov, A., "Pr Magnetic Order and Spin Dynamics in the Cuprates," Chin. J. Phys. 38 (2), 286 (2000).

Lynn, J. W., Skanthakumar, S., "Neutron Scattering Studies of Lanthanide Magnetic Ordering," Handbook on the Physics and Chemistry of Rare Earths (edited by K. A. Gschneider, Jr., L. Eyring, and M. B. Maple) Elsevier Science, in press.

Magid, L. J., Han, Z., Li, Z., Butler. P. D., "Tuning the Contour Lengths and Persistence Lengths of Cationic Micelles: The Role of Electrostatics and Specific lon Binding," J. Phys. Chem. B 104 (29), 6717 (2000).

Magid, L. J., Han, Z., Li, Z., Butler, P. D., "Tuning Microstructure of Cationic Micelles on Multiple Length Scales: The Role of Electrostatics and Specific Ion Binding," Langmuir 16 (1), 149 (1999)

Magid, L. J., Li, Z., Butler, P. D., "Flexibility of Elongated Sodium Dodecylsulfate Micelles in Aqueous Sodium Chloride: A Small-Angle Neutron Scattering Study," Langmuir, in press.

Majkrzak, C. F., "Neutron Applications in Materials Science and Engineering," J. Neutron Res., in press.
Majkrzak, C. F., "Neutron Reflectometry Studies of Thin Films and Multilayered Materials," Acta Phys. Pol. A 96 (1), 81 (1999).

Majkrzak, C. F., Berk, N. F., Krueger, S., Dura, J. A., Tarek, M., Tobias, D., Silin, V. Meuse, C. W., Woodward, J., Plant, A. L., "First-Principles Determination of Hybrid Bilayer Membrane Structure by Phase-Sensitive Neutron Reflectometry," Biophysical $\mathrm{J}$., in press.

Majkrzak, C. F., Berk, N. F., Silin, V., Meuse, C. W., "Experimental Demonstration of Phase Determination in Neutron Reflectometry by Variation of the Surrounding Media," Physica B 283 (1), 248 (2000).

Majkrzak, C. F., Satija, S. K., Berk, N. F., Krueger, S. K., Borchers, J. A., Dura, J. A., Ivkov, R., O'Donovan, K., "Neutron Reflectometry at the NCNR," Neutron News, in press.

Manias, E., Chen, H., Krishnamoorti, R., Genzer, J., Kramer, E. J., Giannelis, E. P., "Intercalation Kinetics of Long Polymers in 2 nm Confinements," Macromol. 33 (21), $7955(2000)$.

Manley, M. E., McQueeney, R. J., Robertson, J. L., Fultz, B., Neumann, D. A., "Phonon Densities of States of -Cerium and -Cerium Measured by Time-of-Flight Inelastic Neutron Scattering," Phil. Mag. Lett. 80 (8), 591 (2000).

Mansky, P., Hawker , C. J., Mays, J., Cook, D. C., "Ordered Diblock Copolymers on Random Copolymer Brushes," Macromol., in press.

Manson, J. L., Huang, Q., Lynn, J. W., Koo, H., Whangbo, M., Bateman, R., Wada, N., Awaga, K., Argyriou, D. N., Miller, J. S., "Long-Range Magnetic Order in $\operatorname{Mn}\left[\mathrm{N}(\mathrm{CN})_{2}\right]_{2}(\mathrm{pyz})\{$ pyz = pyrazine $\}$. Susceptibility, Magnetization, Specific Heat and Neutron Diffraction Measurements and Electronic Structure Calculations," J. Am. Chem. Soc., in press.

Maranzano, B. J., Wagner, N. J., Fritz, G., Glatter, O., "Surface Charge of 3-(Trimethoxysilyl) Propyl Methacrylate (TPM) Coated Stoeber Silica Colloids by Zeta-PALS and SANS," Langmuir, in press.

Margadonna, S., Li, W. Z., Prassides, K., Neumann, D. A., "Rotational Dynamics of $\mathrm{C}_{60}$ in Superconducting $\mathrm{K}_{3} \mathrm{Ba}_{3} \mathrm{C}_{60}$ ”, Chem. Phys. Lett. 322 (6), 472 (2000).

Matejicek, J., Sampath, S., Gnäupel-Herold, T., Prask, H. J., "Processing Effects on Residual Stress in Ni+5\%AI Coatings - Comparison of Different Spraying Methods," in Proceedings of International Thermal Spray Conference 2000, in press.

Matsuda, M.. Lee, Y. S., Greven, M., Kastner, M. A., Birgeneau, R. J., Yamada, K., Endoh, Y., Boni, P., Lee, S. -H., Wakimoto, S., Shirane, G., "Freezing of Anisotropic Spin Clusters in $\mathrm{La}_{1.98} \mathrm{Sr}_{0.02} \mathrm{CuO}_{4}$, Phys. Rev. B 61 (6), 4326 (2000).

Maurer, R. H., Roth, D. R., Fainchtein, R., Goldsten, J. O., Kinnison, J. D., Thompson, A. K., "Portable Real Time Neutron Spectrometry," in Proceedings of the Space Technology and Applications International Forum (STAIF-99), Conference on International Space Station Utilization, in press.

McLaughlin, J. C., Neumann, D. A., Gress, D. L., Livingston, R. A., "Non-Destructive Measurement of Freeze/Thaw Cycles in Cement Paste Using Quasi-Elastic Neutron Scattering," in Proceedings of Third International Conference on Concrete Under Severe Conditions: Environment \& Loading, in press.

Merfeld, G. D., Karim, A., Majumdar, B., Satija, S. K., Paul, D. R., "Interfacial Thickness between Immiscible Bilayers of Poly (phenylene oxide) and Styrenic Copolymers," J. Poly. Sci. Part B, in press.

Merzbacher, C. 1., Barker, J. G., Long, J. W., Rolison, D. R., "The Morphology of Nanoscale Deposits of Ruthenium Oxide in Silica Aerogels," Nanostruct. Mater. 12 (1), 551 (1999).

Meyer, A., Busch, R., Schober, H., "Time-Temperature Superposition of Structural Relaxation in a Viscous Metallic Liquid," Phys. Rev. Lett. 83, 5027 (1999).

Meyer, A., Wuttke, J., Petry, W., "Two-Step Relaxation in a Viscous Metallic Liquid," J. Non-Cryst. Solids 250, 116 (1999).

Michels, A., Weissmüller, J., Wiedenmann, A., Barker, J., "Exchange-Stiffness Constant in Cold-Worked and Nanocrystalline Ni by Elastic Small-Angle Neutron Scattering," J. Appl. Phys. 87, (9) 5953 (2000).

Migler, K. B., Han, C. C., "Pressure Effects on Diblock Copolymers: Optical Birefringence and Neutron Scattering," Macromol., in press.

Mildner, D. F. R., "X-ray and Neutron Optics," in The Handbook of Optics, edited by W M. Gibson, C. A. MacDonald, chapter 6 (Optical Society of America, McGraw-Hill Publishing, New York, NY), in press. 
Millburn, J. E., Green, M. A., Neumann, D. A., Rosseinsky, M. J., "Evolution of the Structure of the $\mathrm{K}_{2} \mathrm{NiF}_{4}$ Phases $\mathrm{La}_{2-x} \mathrm{Sr}_{x} \mathrm{NiO}_{4+}$ With Oxidation State: Octahedral Distortion and Phase Separation (0.2 x 1.0)," J. Solid State Chem. 145 (2), $40 \mathrm{I}$ (1999).

Morkved, L., Chapman, B. R., Bates, F. S., Lodge, T. P., Stepanek, P., Almdal, K., "Dynamics of Ternary Polymer Blends: Disordered, Ordered, and Bicontinuous Microemulsion Phases," Faraday Disc. 112, 335 (1999).

Morkved, T. L., Chapmen, B. R., Bates, F. S., Lodge, T. P., Stepanek, P., Almdal, K., "Dynamics of Ternary Polymer Blends: Disordered, Ordered, and Bicontinuous Microemulsion Phases," Faraday Discuss. 112, 335 (1999).

Nakatani, A. I., "Chain Dimensions in Polysilicate-Filled Poly (Dimethyl Siloxane) (noting)," in International Journal of Thermophysics, $14^{\text {th }}$ Symposium on Thernophysical Properties (Boulder, CO), in press.

Nakatani, A. I., "Phase Separation Kinetics During Shear in Compatibilized Polymer Blends," in Scattering from Polymers, edited by B. S. Hsiao, P. Cebe, D. J. Lohse, ACS Symp. Series 739, (Amer. Chemical Soc., Washington, 2000), p. 405.

Nakatani, A. I., Chen, W., Schmidt, R. G., Gordon, G. V., Han, C. C., "Chain Dimensions in Polysilicate-Filled Poly(Dimethylsiloxane)," Macromol., in press.

Nakatani, A. 1., Ivkov, R., Papanek. P., Yang, H., Gerspacher, M., "Concentration Dependence of Bound Polymer Dynamics by Inelastic Neutron Scattering," ACS PMSE Preprint, in press.

Nakatani, A. I., Ivkov, R., Papanek, P., Yang, H., Gerspacher, M., "Inelastic Neutron Scattering From Filled Elastomers," Rub. Chem. Tech., in press.

Newstein, M. C., Wang, H., Balsara, N. P., Lefebvre, A. A., Shnidman, Y., Watanabe, H., Osaki, K., Shikita, T., Niwa, H., Morishima, Y., "Microstructural Changes in a Colloidal Liquid in the Shear Thinning and Shear Thickening Regimes," J. Chem. Phys. 111 (10), 4827 (1999).

Nico, J. S., "Establishing International Collaborations: Examples of Mid-Size Collaborations," in Report of the American Association for the Advancement of Science and the AAAS Web Site, in press.

Nico, J. S., Abdurashitov, J. N., Gavrin, V. N., Girin, S. V., Gorbachev, V. V., Ibragimova, T. V., Kalikhov, A. V., Khairnasov, N. G., Knodel, V. N., Mirmov, I. N., Shikhin, A. A., Verentenkin, E. P. Vermul, V. M., Yants, V. E., Zatsepin, G. T., Bowles, T. J., Teasdale, W. A., Wark, D. L., Cherry, M. L., Cleveland, B. T., Davis, R, Lande, K., Wildenhain, P. S., Elliott, S. R., Wilkerson, J. F., "Solar Neutrino Results From SAGE," in Proceedings of the $10^{\text {th }}$ International Baksan School: Particles and Cosmology, in press.

Nisato, G., Ivkov, R., Amis, E. J., "Size Invariance of Polyelectrolyte Dendrimers," Macromol. 33 (11), 4172 (2000).

Norman. B. R., Becker, D. A. "Determination of Ultratrace Levels of Mercury in Three SRMs by Combustion RNAA," J. Radioanal. Nucl. Ch. 245 (1), 91 (2000).

Norman, B. R., lyengar, G. V., "Further Applications of Pre-irradiation Combustion and Neutron Activation Analysis Technique for the Determination of Iodine in Food and Environmental Reference Materials: Merits and Demerits," Fresen. J. Anal. Chem., in press.

Olson, D. H., Khosrovani, N., Peters, A., Toby, B., "The Crystal Structure of Dehydrated CsZSM-5 (5.8A1): Evidence for Non-Random Aluminum Distribution," J. Phys. Chem. B $104(20), 4844$ (2000).

Orts, W. J., Glenn, G. M., "Reducing Soil Erosion Losses with Small Applications of Biopolymers." in Biopolymers: Utilizing Natures Advanced Materials, edited by R. V. Greene and S. H. Imam (ACS Symposium Series, Washington, DC, 1998), in press.

Oslanec, R., Composto, R. J., Vlcek. P., "Black Copolymer Adsorption at the Polymer Melt/Substrate Interface: The Effect of Matrix Competition," Macromol. 33 (6), 2200 (2000).

Pan, Y. -M., Page, R. A., Long, G. G., Krueger, S., "The Role of Zirconia Addition in Pore Development and Grain Growth in Alumina Compacts," J. Mater. Res. 14 (12), 4602 (1999).

Papanek, P., Fischer, J. E., Sauvajol, J. L., Dianoux, A. J., McNeillis, P. M., Mathis. C., Francois, B., "Low-Frequency Phonons in Pristine and Doped Phases of Polyacetylene: An Inelastic Neutron Scattering Study," J. Chem. Phys., in press.

Park, S., Parise, J. B., Gies, H., Liu, H., Grey, C. P., Toby, B. H., "A New Porous Lithosilicate With a High Ionic Conductivity and Exceptional Ion-Exchange Capacity," J. Amer. Chem. Soc. 122, 11023 (2000).
Parr, R. M. Kawamur, H., Iyengar, G. V., "Acquisition of Improved Reference Values for Cesium, Iodine, Strontium, Thorium, and Uranium in Selected NIST Reference Materials," Biol. Trace Elem. Res. 71 (2), 5 (1999).

Paul, R. L., Chen-Mayer, H. H., Lindstrom, R. M., "Characterization of Hydrogen in Concrete by Cold Neutron Prompt Gamma-ray Activation Analysis and Incoherent Scattering," in Materials Research Society Fall 1999 Conference on Nondestructive Methods and Materials Characterization, edited by G.Y. Baaklini, N. Meyendorf, T. E. Matikas, R. S. Gilmore (Matls. Res. Soc., Warrendale, PA, 2000), p.I75.

Paul, R. L., Lindstrom, R. M., "Prompt Gamma-Ray Activation Analysis: Fundamentals and Applications," J. Radioanal. Nucl. Ch. 243 (1), 181 (2000).

Paul, R. L., Simons, D. S., "Neutron Activation Analysis for Calibration of Phosphorus Implantation Dose," in 2000 International Conf. on Characterization and Metrology for ULSI Technology, edited by D. G. Seiler, A. C. Diebold, W. M. Bullies, T. J. Shaffner, R. McDonald, E. J. Walters (AIP Press, Woodbury, NY), in press.

Paul, R.L., "Determination of Nitrogen in Some Reference Materials by Cold Neutron Prompt Gamma-ray Activation Analysis," J. Radioanal. Nucl. Ch., in press.

Paul, R.L., "Determination of Phosphorus in Steels by Radiochemical Neutron Activation Analysis," J. Radioanal. Nucl. Ch., in press.

Paul, R.L., "Measurement of Phosphorus in Metals by Radiochemical Neutron Activation Analysis,” J. Radioanal. Nucl. Ch. 245 (I), 1 I (2000).

Paul, R.L., "Nitrogen in Some Reference Materials by Cold Neutron Prompt Gamma-ray Activation Analysis," The Analyst, in press.

Pearce, J. V., Azuah, R. T., Stirling, W. G., Dimeo, R. M., Sokol, P. E., Adams, M A., "High-Resolution Measurements of the Temperature Dependence of the Roton Energy of Superfluid “He," Physica B 276, 822 (2000).

Peter, S., Altorfer, F., Buhrer, W., Lutz, H. D., "Sr$(\mathrm{OH}) \mathrm{Br}$, a Metal Hydroxide Halide With Unusual Properties," J. Solid State Chem. 151 (2), 267 (2000).

Plazanet, M., Johnson, M. R., Gale, J. D., Yildirim, T., Kearley, G. J., Trommsdorff, H. P., Fernandez-Diaz, M. T., Sanchez-Portal, D., Artacho, E., Soler, J. M., Ordejon, P., Garcia, A., "The Structure and Dynamics of Crystalline Durene by Neutron Scattering and Numerical Modeling using Density Functional Methods," Chem. Phys., in press.

Pochan, D. J., Lin, E. K., Wu, W. L., Satija, S. K., "Thermal Expansion of Supported Thin Polymer Films: A Direct Comparison of Free Surface vs. Total Confinement," Macromol., in press.

Pochan, D. J., Lin, E. K., Wu, W. L., Satija, S. K., Cheng, S. Z. D., "Thermal Expansion and Glass Transition Behavior of Thin Polymer Films with and without a Free Surface via Neutron Reflectometry," in Dynamics in Small Confining Systems IV, edited by J. M. Drake, G. S. Grest, J. Klafter, R. Kopelman, MRS Symp. Series 543, (Materials Research Society, Warrendale, PA, 1999), in press.

Pollard, M., Tsui, O. K. C., Russell, T. P., Ruzette, A. V., Mayes, Gallot, Y., "Some Thermodynamics Considerations of the Lower Critical Ordering of Diblock Copolymers," in Scattering From Polymers: Characterization by X-Rays, Neutrons, and Light, edited by P. Cebe, B. S. Hsiao, D. J. Lohse (American Chemical Society, Washington, DC 2000), p. $26 \mathrm{I}$

Pomme, S., Simonits, A., Lindstrom, R. M., DeCorte, F., Robouch, P., "Determination of Burnup Effects Prior to Reactor Neutron Field Characterization," J. Radioanal. Nucl. Ch. 245 (1), 223 (2000).

Prask, H. J., Brand, P. C., Gnäupel-Herold, T., Hicho, G. E., "Determination of Residual Stresses Using Neutron Diffraction: Tank-Car Welds and Rail Slices," in ASNT's Topics on Nondestructive Evaluation Series, in press.

Prask, H. J., Gnäupel-Herold, T., Clark, A. V., Hehman, C. S., Nguyen, T. N., "Neutron and Ultrasonic Determination of Residual Stress in an Aluminum Ring-Plug," in Review of Progress in Quantative NDE 19B, edited by D. O. Thompson, D. E., Chimenti, AIP 509, (AIP, Melville, NY), p. 243.

Prince, E., "Neutron Scattering Instrumentation: A Tutorial Review" App. Spectrosc. Rev 34 (3), 159 (1999).

Qi, S., Chakraborty, A. K., Wang, H., Lefebvre, A. A., Balsara, N. P., Shaknovich, E. I., Xenidou, M., Hadjichristidis, N., "Microphase Ordering in Melts of Randomly Grafted Copolymers," Phy. Rev. Lett., 82 (14), 2896 (1999).

$\mathrm{R}$ (3) (MNiF, )-Ni-Il-F-IV ( $\mathrm{M}=\mathrm{Fe}, \mathrm{Co}, \mathrm{Cu}, \mathrm{Zn})$, and ( $\mathrm{MNiF}$ )-Ni-II-F-II $(\mathrm{M}=\mathrm{Co}, \mathrm{Cu})$," Cr. Acad. Sci. II C 2 (11), 557 (1999).

Reeber, R. R., Wang, K., "Lattice Parameters and Thermal Expansion of GaN," J. Mater Res. 15 (1), 40 (2000). 
Reisner, B. A., Lee. Y., Hanson, J. C., Jones, G. A., Parise, J. B., Corbin, D. R., Toby, B. H., Freitag, A.. Larese, J. Z., Kahlenberg, V., "Understanding Negative Thermal Expansion and "Trap Door" Cation Relocations in Zeolite Rho," Chem. Comm., in press.

Reisner, B. A., Tripathi, A., Parise, J. B., " $\mathrm{Na}_{8}\left[\mathrm{Zn}_{4} \mathrm{Ge}_{8} \mathrm{O}_{18}(\mathrm{OH})_{12}\right] \cdot 2 \mathrm{H}_{2} \mathrm{O}$ : An InterruptedFramework Zincogermanate Built From $\left[\mathrm{GeO}_{3}(\mathrm{OH})\right]$ and $\left[\mathrm{Ge}_{2}(\mathrm{OH})_{2}\right]$ Units," J. Mater. Chem., in press.

Ren. Y., Lodge, T. P., Hillmyer, M. A., "Synthesis, Characterization, and Interaction Strengths of Difluorocarbene-Modified Polystyrene-Polysoprene Block Copolymers," Macromol. 33 (3), 866 (2000).

Rich, D. R., Fan, S., Gentile, T. R., Hussey. D., Jones, G. L., Neff, B., Snow, W. M., Thompson, A. K., "Polarized "He Neutron Spin Filter Development and Application at Indiana University and NIST," Physica B, in press.

Richardson, R. M., Pelton, R H., Cosgrove, T., Zhang, J., "A Neutron Reflectivity Study of Poly(N-Isopropylacrylamide) at the air Water Interface With and Without Sodium Dodecylsulfate," Macromol. 33 (17), 6269 (2000).

Rijssenbeek, J. T., Huang, Q., Erwin, R. W., Zandbergen, H. W., Cava, R. J., "The Crystal Structure of $\mathrm{Ba}_{3} \mathrm{CuRu}_{2} \mathrm{O}_{9}$ and Comparison to $\mathrm{Ba}_{3} \mathrm{MRu}_{2} \mathrm{O}_{9}(\mathrm{M}=\ln , \mathrm{Co}, \mathrm{Ni}$, and $\mathrm{Fe})$," J. Solid State Chem. 146 (1), 65 (1999).

Rizi, R. R., Roberts, D. A., Dimitrov, I. E., Lipson, D. A., Gentile, T. R., Jones, G., Thompson, A., Gefter, W. B., Schnall, M. D., Leigh, J. S., "Ventilation/Perfusion of Lung Parenchyma Using Hyperpolarized "He Gas and Arterial Spin-Tagging," J. Magnetic Resonance Imaging, in press.

Rosenkranz, S., Osbom, R., Mitchell, J. F., Vasiliu-Doloc, L., Lynn, J. W., Sinha, S. K. "Low-energy Spin Wave Excitations in the Bilayer Manganite $\mathrm{La}_{12} \mathrm{Sr}_{1.8} \mathrm{Mn}_{2} \mathrm{O}_{7}$," J. Appl. Phys. 87 (9), 5816 (2000).

Rosenkranz, S., Osborn, R., Mitchell, J. F., Vasiliu-Doloc, L., Lynn, J. W., Sinha, S. K., "Spin Correlations of the Magnetoresistive Bilayer Manganite $\mathrm{La}_{1.2} \mathrm{Sr}_{1.8} \mathrm{Mn}_{2} \mathrm{O}_{7}$," Int. J. Mod. Phys. B 13 (29), 3820 (1999).

Rosov, N., Rathgeber, S., Monkenbusch, M., "Neutron Spin Echo Spectroscopy at the NIST Center for Neutron Research," in Scattering From Polymers Characterization by X-rays, Neutrons and Light, edited by P. Cebe, B. S. Hsiao, D.J. Lohse. ACS Symp. Series 739, (American Chemical Society, Washington, DC, 2000), p.I03.

Rossbach, M., Zeisler. R, “Certified Reference Materials for Microanalytical Methods," in Reference Materials for Chemical Analysis - Certification, Availability, and Proper Usage, edited by M. Stoeppller, W. R. Wolf, P. Jenks, chapter 4.3, (John Wiley and Sons, Inc., New York, NY), in press.

Rush, J. J., Udovic, T. J., Berk, N. F., Richter, D., Magerl, A., "Excited-State Vibrational Tunnel Splitting of Hydrogen Trapped by Nitrogen in Niobium," Europhys. Lett. 48 (2), 187 (1999).

Salamat, G., de Uries, R., Kaler, E. W., Satija, S., Sung, L., "Undulations in Salt-Free Charged Lamellar Phases Detected by Small Angle Neutron Scattering and Neutron Reflectivity," Langmuir 16 (I), 102 (2000)

Sankar, S., Dender, D., Borchers, J. A., Smith, D. J., Erwin, R. W., Kline, S. R., Berkowitz, A. E., "Magnetic Correlations in Non-Percolated

Santoro, A., "Neutron Crystallography at NBS/NIST," ACA Newsletter, in press.

Santoro, A., Natali Sora, I., Huang, Q., "Bond Valence Analysis of BaRuO(3)," J. Solid State Chem. 151 (2), 245 (2000).

Sayer, E. V., Yener, A. K., Joel, E. C., Blackman, M. J., Özbal, H., "Stable Lead Isotope Studies of Black Sea Anatolian Ore Sources and Related Bronze Age and Phrygian Artifacts from Nearby Archaeological Sites. Appendix: New Central Taurus Ore Data," Archaeometry, in press.

Schmidt, G., Nakatani, A. I., Butler, P. D., Karim, A., Han, C. C., "Shear Orientation of Viscoelastic Polymer-Clay Solutions Probed by Flow Birefringence and SANS," Macromol. 33 (20), 7219 (2000).

Schmidt, G., Nakatani, A. I., Butler, P. D., Karim, A., Han, C. C., "Shear Orientation of Viscoelastic Polymer-Clay Solutions Probed by Flow Birefringence and SANS," in Proceedings of the XIIIth Congress of Rheology 2000, in press.

Schmidt-Winkel, P., Glinka, C. J., Stucky, G. D., "Microemulsion Templates for Mesoporous Silica," Langmuir 16 (2), 356 (2000).

Schober, H., Dianoux, A. J., Cook, J. C., Mezei, F., "Upgrade of the INS Cold Neutron Time-of-Flight Spectrometer," Physica B 276, 164 (2000).

Schober, H., Losert, C., Mezei, F., Cook, J. C., "Chopper Cascades: An Analytical Treatment of the Contamination Problem," J. Neutron Res., in press.
Schreyer, A., Schmitte, T., Siebrecht, R., Bodeker, P., Zabel, H., Lee, S. H., Erwin, R. W., Majkrzak, C. F., Kwo, J., Hong, M., "Neutron Scattering on Magnetic Thin Films: Pushing the Limits," J. Appl. Phys. 87 (9), 5443 (2000).

Schulz, J. C., Warr, G. G., Butler, P. D., Hamilton, W. A., "Adsorbed Layer Structure of Cationic Surfactants on Quartz," Phys. Rev. E, in press.

Schulz, J. C., Warr, G. G., Hamilton, W. A., Butler, P. D., "A New Model for Neutron Reffectometry of Adsorbed Surfactant Aggregates," J. Phys. Chem. B 103 (50), 11057 (1999).

Seehra, M. S., Babu, V. S., Manivannan, A., Lynn, J. W., "Neutron Scattering and Magnetic Studies of Ferrihydrite Nanoparticles" Phys. Rev. B 61 (5), 3513 (2000).

Sharov, V. A., Xiao, Q. F., Ponomarev, I. Y., Mildner, D. F. R., Chen-Mayer, H. H., "Cold Neutron Microprobe for Materials Analysis Using Tapered Capillary Optics," Rev. Sci. Instrum., 71 (9), 3247 (2000).

Shen, C. P., Chacon, L. C., Rosov, N., Elder, S. H., Allman, J. C., Bartlett, N., "The

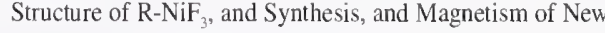

Shen, G. Q., Toulouse, J., Beaufils, S., Bonello, B., Hwang, Y. H., Finkel, P., Hernandez, J., Bertault, M., Maglione, M., Ecolivet, C., Cummins, H. Z., "Experimental Studies of the Liquid-Glass Transition in Trimethylheptane," Phys. Rev. E 62 (1), 783 (2000).

Shirane, G., Gehring P. M., "Soft Phonon Anomalies in Relaxor Ferroelectrics," J. Phys. Soc. Jpn., in press.

Sitepu, H., Prask, H. J., Vaudin, M. D., "Texture Characterization in X-ray and Neutron Powder Diffraction Data Using the Generalized Spherical-Harmonic," in Proceedings of the Annual Conference on Applications of X-ray Analysis, in press.

Skripov, A. V., Cook, J. C., Udovic, T. J., Kozhanov, V. N., "Quasielastic Neutron Scattering Study of Hydrogen Motion in C15-Type $\mathrm{HfMo}_{2} \mathrm{H}_{0.26}$ " Phys. Rev. B, in press.

Skripov, A. V., Udovic, T. J., Huang, Q. Z., Cook, J. C., Kozhanov, V. N., "Neutron Diffraction Study of the Location of Deuterium in the Deuterium-Stabilized $\mathrm{ZrTi}_{2} \mathrm{D}_{3.83}$ Phase," J. Alloy Compd. 311 (2), 234 (2000).

Snow, W. M., Bazhenov, A., Blessinger, C. S., Bowman, J. D., Chupp, T. E., Coulter, K. P., Freedman, S. J., Fujikawa, B. K., Gentile, T. R., Greene, G. L., Hansen, G., Hogan,G. E., Ishimoto, S., Jones, G. L., Knudson, J. N., Kolomenski, E., Lamoreaux, S. K., Leuschner, M. B., Masaike, A., Masuda, Y., Matsuda, Y., Morgan, G. L., Morimoto, K., Morris, C. L., Nann, H., Penttila, S. l., Pirozhkov, A., Pomeroy, V. R., Rich, D. R., Serebrov, A., Sharapov, E. I., Smith, D. A., Smith, T. B., Welsh, R. C., Wietfeldt, F. E., Wilburn, W. S., Yuan, V. W., and Zerger, J., "Measurement of the Parity Violating Asymmetry A in $n+p \rightarrow d+$," Nucl. Instrum. Meth. A 440 (3), 729 (2000).

Snow, W. M., Chowdhuri, Z., Dewey, M. S., Fei, X., Gilliam, D. M., Greene, G. L., Nico, J. S., Wietfeldt, F. E. "A Measurement of the Neutron Lifetime by Counting Trapped Protons," Nucl. Instr. Meth. A 440 (3), 528 (2000),

Sokolov, M. A., Spooner, S. E., Odette, G. R., Wirth, B. D., Lucas, G. E., "SANS Study of High-Copper RPV Welds in lrradiated and Annealed Conditions," in Effects of Radiation on Materials: 18 International Symposium, ASTM STP 1325, edited by R. K. Nanstad, M. L. Hamilton, F. A. Garner, and A. S. Kumar (ASTM, West Conshohocken, PA, 1999), in press.

Stanka, M. B., Adams, J. M., Eisenhauer, C. M., "Proton Recoil Measurements of the ${ }^{252} \mathrm{Cf}$ Fission Neutron Leakage Spectrum from an Iron Sphere," Nucl. Sci. Eng. 134 (1), 68 (2000).

Su, T. J., Green, R. J., Wang, Y., Murphy, E. F., Lu, J. R., Ivkov, R., Satija, S. K., "Adsorption of Lysozyme Onto the Silicon Oxide Surface Chemically Grafted With a Monolayer of Pentadecyl-1-ol," Langmuir 16 (11), 4999 (2000).

Subramanian, M. A., Li, D., Duan, N., Reisner, B. A., Sleight, A W., "High Dielectric Constant in $\mathrm{ACu}_{3} \mathrm{Ti}_{4} \mathrm{O}_{12}$ and $\mathrm{ACu}_{3} \mathrm{Ti}_{3} \mathrm{FeO}_{12}$ Phases," J. Solid State Chem. 151 (2), $323(2000)$

Sung, L., Karim, A., Douglas, J. F., Han, C. C., "Modification of Thin Film Phase Separation by a Surfactant," Macromol., in press.

Tarek M., Martyna, G. J., Tobia, D. J., "Amplitude and Frequencies of Protein Dynamics: Analysis of Discrepancies Between Neutron Scattering and Molecular Dynamics Simulations," J. Am. Chem. Soc. 122 (42), 10450 (2000).

Tarek, M., Tobias, D. J., "The Dynamics of Protein Hydration Water: A Quantitative Comparison of Molecular Dynamics Simulations and Neutron Scattering Experiments," Biophys. J., in press.

Thomas, J. J., Jennings, H. M., Allen, A. J., "The Surface Area of Hardened Cement Paste as Measured by Various Techniques," Con. Sci. Eng., in press. 
Thomas, J. J., Neumann, D. A., Fitzgerald, S. A., Livingston, R. A., "The State of Water in Hydrating Tricalcium Silicate and Portland Cement Pastes as Measured by QuasiElastic Neutron Scattering," J. Am. Ceram. Soc., in press.

Thurn-Albrecht, T., DeRouchey, J., Stafford, C., Huang, E., Bal, M., Tuominen, M., Russell, T. P., Hawker, C. J., "Nanoscopic Templates From Oriented Block Copolymer Films," Adv. Mater. 12 (11), 787 (2000).

Toby, B. H., "Diffraction Analysis and Crystallographic Structurc Determination," Handbook of Ceramic Engineering, ed. M. N. Rahaman, in press.

Toby, B. H., "The Classification of Powder Diffraction Data," International Tables for Crystallography, in press.

Toby, B. H., Corbin, D. R., Dartt, C. B., Davis, M. E., Freitag, A., Gies, H., Grey, C. P., Hanson. J. C., Johnson, G. M., Jones, G., Kahlenberg, V., Khosrovani, N., Larese, J. Z., Lee, Y., Liu, H., Parise, J. B., Park, S. -H., Reisner, B. A., Tripathi, A., "Structural Studies of Zeolitic Materials With the NIST BT-1 Neutron Powder Diffractometer," Neutron News, in press.

Toby, B. H., Khosrovani, N., Dartt, C. B., Davis, M. E., Parise, J. B., "Structure-Directing Agents and Stacking Faults in the CON System: A Combined Crystallographic and Computer Simulation Study," Micropor. Mesopor. Mat. 39 (1), 77 (2000).

Topp, A., Bauer, B. J., Prosa, T. J., Scherrenberg, R., Amis, E. J., "Size Change of Dendrimers in Concentrated Solution," Macromol. 32 (26), 8923 (1999).

Trevino, S. F., Ivkov, R., Matyas, G., Lebeda, F. J., "Small-Angle Neutron Scattering of Bilayer Vesicles Made with Synthetic Phospholipids," in Biomedical Materials-Drug Delivery, Implants and Tissue Engineering, edited by Neenan, Marcolongo, Valentini, MRS 550 (1999), p. 83.

Trewhella, J., Krueger, J. K., "Small-Angle Solution Scattering Reveals Information on Conformational Dynamics in Calcium-Binding Proteins and in Their Interactions with Regulatory Targets," in Calcium Binding Proteins Protocols, edited by H. Vogel, J. M Walker, (Humana Press, I999), in press.

Trollsas, M., Athoff, B., Claesson, H., Hedrick, J. L., Pople, J. A., Gast, A. P., "Six Isomers of Dendrimer-Like Star Polymers, Design and Synthesis" Abstr. PAP Amer. Chem. Soc. 217 U459 (1999).

Trollsas, M., Atthof, B., Wursch, A., Hedrick, J. L., Pople, J. A., Gast, A. P., "Constitutional Isomers of Dendrimer-Like Star Polymers: Design, Synthesis, and Conformational and Structural Properties," Macromol. 33 (17), 6423 (2000).

Trollsas, M., Claesson, H., Atthoff, B., Hedrick, J. L., Pople, J. A., Gast, A. P., "Conformational and Structural Properties of High Functionality Dendrimer-Like Star Polymers Synthesized From Living Polymerization Techniques," Macromol. 153, 87 (2000),

Troyanchuk, I. O., Kasper, N. V., Khalyavin, D. D., Tonoyan, A. A., "Structure, Phase Transitions, and Magnetotransport Properties of $\mathrm{TbBaCo}_{2.8} \mathrm{Fe}_{\mathbf{x}} \mathrm{O}_{68}(\mathrm{x} \leq 0.5)$ Perovskites," Phys. Rev B., in press.

Troyanchuk, I. O., Khalyavin, D. D., Solovykh, T. K., Szymczak, H., Huang, Q., Lynn, J. W., "Magnetic and Crystal Structure Phase Transitions in $\mathrm{R}_{1-x} \mathrm{Ba}_{x} \mathrm{CoO}_{3-y}(\mathrm{R}=\mathrm{Nd}, \mathrm{Gd})$," J. Phys. Condens. Mat. 12 (I 1), 2485 (2000).

Troyanchuk, 1. O., Khaylavin, D. D., Lynn, J. W., Erwin, R. W., Huang, Q., Szymczak, H., Szymczak, R., Baran, M., "Magnetic Phase Diagrams of the $\mathrm{Ln}\left(\mathrm{Mn}_{+} \mathrm{Co}_{*}\right) \mathrm{O}_{3}$ (Ln=Eu, Nd, Y) Systems," J. Appl. Phys. 88 (1), 360 (2000).

Truong, M. T., Walker, L. M., "Controlling the Shear-Induced Structural Transition of Rodlike Micelles Using Nonionic Polymer," Langmuir, in press.

Tsai, A. M., Neumann, D. A., Bell, L. N., "Molecular Dynamics of Lysozymc," Biophys. $\mathrm{J}$., in press.

Tung, C. -S., Wall, M. E., Gallagher, S. C., Trewhella, J., "A Molecular Model of the Troponin C/Troponin I Interaction Using Constraints from X-Ray Crystallography, NMR, Small Angle Scattering, Cross-Linking and FRET," Protein Science, in press.

Udovic, T. J., Huang, Q., Erwin, R.W., Hjörvarsson, B., Ward, R. C. C., "The Structural Symmetry of YD ${ }_{3}$ Epitaxial Thin Films," Phys. Rev. B 61 (19), 12701 (2000).

Udovic, T. J., Huang, Q., Karmonik, C., Rush, J. J., "The Structural Ordering and Dynamics of $\mathrm{LaH}_{3-x}$," J. Alloy Compd. 293, 113 (1999).

Udovic, T. J., Huang, Q., Rush, J. J., "Hydrogen and Dcuterium Site Scparation in FCCBased, Mixed-lsotope, Rare-Earth Hydrides," Phys. Rev. B 61 (I0), 6611 (2000).

Udovic, T. J., Karmonik, C., Huang, Q., Rush, J. J., Vennstrom, M., Andersson, Y., Flanagan, T. B., "Comparison of the Dynamics of Hydrogen and Deuterium Dissolved in Crystalline $\mathrm{Pd}_{9} \mathrm{Si}_{2}$ and $\mathrm{Pd}_{3} \mathrm{P}_{08}$," J. Alloy. Compd., in press.
Udovic, T. J., Yildirim, T., Karmonik, C., Huang, Q., "The Applications of Neutron Scattering Methods to the Study of High-Temperature Protonic Conductors," in Solid State Ionics $V$. edited by G.A. Nazri, C. Julien, A. Rougier, MRS Symp. Series 548 (MRS, Pittsburgh, 1999), p. 593.

Unguris, J., Tulchinsky, D., Kelley, M. H., Borchers, J. A., Dura, J. A., Majkrzak, C. F., Hsu, S. Y., Loloee, R., Pratt, W. P., Bass, J., "Magnetic Depth Profiling $\mathrm{Co} / \mathrm{Cu}$ Multilayers to Investigate Magnetoresistance," J. Appl. Phys. 87 (9), 6639 (2000).

van der Zaag, P. J., Feiner, L. F., Wolf, R. M., Borchers, J. A., Ijiri, Y., Erwin, R. W., "The Blocking and Neel Temperature in Exchange Biased $\mathrm{Fe}_{3} \mathrm{O}_{4} / \mathrm{CoO}$ Multilayers," Physica B 276, 638 (2000).

van der Zaag, P. J., ljiri, Y., Borchers, J. A., Feiner, L. F., Wolf, R. M., Gaines, J. M., Erwin, R. W., Verheijen, M. A., "Difference Between Blocking and Neel temperatures in the Exchange Biased $\mathrm{Fe}_{3} \mathrm{O} / \mathrm{CoO}$ System," Phys. Rev. Lett. 84 (26), 6102 (2000).

Vasiliu-Doloc, L., Osborn, R., Rosenkranz, S., Mesot, J., Mitchell, J. F., Sinha, S. K., Seeck, O. H., Lynn, J. W., Islam, Z., "Polaron Ordering in Ferromagnetic Colossal Magnetoresistive Oxides," Int. J. Mod. Phys., in press.

Vasiliu-Doloc, L., Rosenkranz, S., Osborn, R., Sinha, S. K., Lynn, J. W., Mesot, J., Seeck, O., Lee, W. K., Mitchell, J. F., "Charge Melting and Polaron Collapse in $\mathrm{La}_{12} \mathrm{Sr}_{1.8} \mathrm{Mn}_{2} \mathrm{O}_{7}$ " Phys. Rev. Lett. 83 (21), 4393 (I999).

Vigild, M.E., Chu, C., Sugiyama, M., Chaffin, K. A., Bates, F. S., "Influence of Shear on the Alignment of a Lamellae Forming Pentablock Copolymer," Macromol., in press.

Wagner, N. J., Butera, R., "Shear Distortion and Relaxation Dynamics of Colloidal Crystals lnvestigated by SANS Time Slicing" Phys. Rev. E, in press.

Wakimoto, S., Birgeneau, R. J., Kastner, M. A., Lee, Y. S., Erwin, R., Gehring, P. M., Lee, S. -H., Fujita, M., Yamada, K., Endoh, Y., Hirota, K., Shirane, G., "Direct Observation of a One-Dimensional Static Spin Modulation in Insulation $\mathrm{La}_{1.95} \mathrm{Sr}_{0.05} \mathrm{CuO}_{4}$, Phys. Rev. B 61 (5), 3699 (2000).

Wakimoto, S., Shirane, G., Endoh, Y., Hirota, K., Ueki, S., Yamada, K., Birgeneau, R. J., Kastner, M. A., Lee, Y. S., Gehring, P. M., Lee, S. -H., "Observation of Incommensurate Magnetic Correlations at the Lower Critical Concentration for Superconductivity in $\mathrm{La}_{2-x} \mathrm{Sr}_{x} \mathrm{CuO}_{4}$ (x=0.05)," Phys. Rev. B 60 (2), R769 (1999),

Wakimoto, S., Yamada, K., Ueki, S., Shirane, G., Lee, Y. S., Lee, S. -H., Kastner, M. A., Hirota, K., Gehring, P. M., Endoh, Y., Birgeneau, R. J., "Neutron Scattering Study of Elastic Magnetic Signals in Superconducting $\mathrm{La}_{1.94} \mathrm{Sr}_{0.06} \mathrm{CuO}_{4}$," J. Phys. Chem. Solids 60 (8), 1079 (1999).

Wall, M. E., Gallagher, S. C., Trewhella, J., "Large-Scale Shape Changes in Proteins and Macromolecular Complexes" Annual Reviews of Physical Chemistry, in press.

Wang, H. Y., Hwang, S. R., Li, W. -H., Lee, K. C., Lynn, J. W., Luo, H. M., Ku, H. C., "Variation in the Structural and Magnetic Propcrties Induced by La-doping in $\operatorname{Pr}\left(\mathrm{Ba}_{1 \cdot-\mathrm{L}} \mathrm{La}_{x}\right)_{2} \mathrm{Cu}_{3} \mathrm{O}_{7+y}$ " Phys. Rev. B, in press.

Washburn, N. R., Lodge, T. P., Bates, F. S., "Ternary Polymer Blends as Model Surfactant Systems," J. Phys. Chem. B 104 (30), 6987 (2000)

Welp, K. A., Co, C, Wool, R. P., "Improved Reflectivity Fitting Using SERF: Spreadsheet Environment Reflectivity Fitting," J. Neutron Res., in press.

Welp, K. A., Wool, R. P., Agrawal, G., Satija, S. K., Pispas, S., Mays, J., “Direct Observation of Polymer Dynamics: Mobility Comparison Between Central and End Section Monomers," Macromol. 32 (15), 5127 (1999).

Wesley, R. D., Cosgrove, T., Thompson, L., "Interactions of Star Polymers With Surfactants,' Langmuir 15 (24), 8376 (1999).

Wesley, R. D., Cosgrove, T., Thompson, L., Armes, S. P., Billingham, N. C., Baines, F. L., "Hydrodynamic Layer Thickness of a Polybase Brush in the Presence of Salt," Langmuir 16 (10), 4467 (2000).

White, D. C. C., Wu, W. L., Pu. Y., Rafailovich, M., Sokolov, J., "Probe Segregation within an Ultra-Thin Polystyrene Film Studied by X-ray, Neutron Reflectivity and SIMS and Tg Determination of Polystyrene on a Quartz Substrate by X-ray Reflectivity," Macromol., in press.

Wilemski, G., "Neutron Scattering From Aerosols: Intraparticle Structure Factor, Guinier Analysis of Particle Speed, and Crossed Beam Kinematics," Phys. Rev E 61 (1), $557(2000)$

Wirth, B. D., Odette, G. R., Pavinich, W.A., Lucas, G. E., Spooner, S. E., "Small Angle Neutron Scattering Study of Linde 80 RPV Welds," in Effects of Radiation on Materials: $18^{\text {th }}$ International Symposium, ASTM STP 1325, edited by R. K. Nanstad, M. L. Hamilton, F. A. Garner, and A. S. Kumar (West Conshohocken, PA, 1999), in press. 
Won, Y. Y., Davis, H. T., Bates, F. S., Agamalian, M., Wignall, G. D., "Segment Distribution of the Micellar Brushes of Poly(Ethylene Oxide) via Small-Angle Neutron Scattering," J. Phys. Chem. B 104 (30), 7134 (2000).

Won, Y. Y., Davis, H. T., Bates, F. S., Agamalian, M., Wignall, G. D., "Segment Distribution of the Micellar Brushes of Poly(Ethylene Oxide) via Small-Angle Neutron Scattering," J. Phys. Chem. B 104 (38), 9054 (2000).

Wong-Ng. W., Huang, Q., Lavin, I., Kaduk, J. A., Dillingham, J., Huagan, T., Suh, J., Cook, L. P., "Crystal Chemistry and Phase Equilibria of Selected $\mathrm{SrO}-\mathrm{R}_{2} \mathrm{O}_{3}-\mathrm{CuO}_{x}$ and Related Systems, R=Lanthanides and Yttrium." J. Solid State Chem., in press.

Wong-Ng, W.. Kaduk, J. A., Huang, Q., Roth, R. S., "Crystal Structure and X-Ray Diffraction Pattern of the Monoclinic $\operatorname{Sr}\left(\mathrm{Bi}_{23} \mathrm{Ca}_{1 / 3}\right) \mathrm{O}_{3}$, J. Solid State Chem., in press.

Wu, S. Y., Hwang, S. R., Li, W. H., Lee, K. C., Lynn, J. W., Liu, R. S., "Jahn-Teller Distortion, Charge Ordering, and Magnetic Transitions in Ca-Doped $\mathrm{NdMnO}_{3}$," Chinese J. Phys. 38 (2), 354 (2000).

Wu, S. Y., Kuo, C. M., Wang, H. Y., Li, W. H., Lee, K. C., Lynn, J. W., Liu, R. S., "Magnetic Structure and Spin Reorientation of the $\mathrm{Mn}$ Ions in $\mathrm{NdMnO}_{3}$ " J. App. Phys. 87 (9), 5822 (2000).

Wu, W. L., Lin, E. K., Lin, Q., Angelopolous, M., "Small-Angle Neutron Scattering Measurements for the Characterization of Lithographically Prepared Structures," in Proceedings of the 2000 Intl. Conf. on Characterization and Metrology for ULSI Technology, in press.

Wu, W. L., Wallace, W. E., Lin, E. K., Lynn, G. W., Glinka, C. J., Ryan, E. T., Ho, H. M., "Properties of Nanoporous Silica Thin Films Determined by High Resolution X-Ray Reflectivity and Small-Angle Neutron Scattering," J. Appl. Phys. 87 (3), 1193 (2000).

Wu. W.. Lin, E. K., Jin, C., Wetzel, J. T. A., "Three-Phase Model for the Structure of Porous Thin Films Determined by X-ray Reflectivity and Small Angle Neutron Scattering," in Proceedings of the Materials Research Society Meeting (April 2000), in press.

Wu, W.-L., Lin, E. K., Lin, Q., Angelopolous, M., "Small Angle Neutron Scattering Measurements of Nanoscale Lithographic Features," Science (in press).

Wuensch, B. J., Eberman, K. W., Heremans, C., Ku, E. M., Onnerud, P., Yeo, E. M., Haile, S. M., Stalick, J. K., Jorgensen, J. D., "Connection Between OxygenIon Conductivity of Pyrochlore Fuel-Cell Materials and Structural Change with Composition and Temperature," Solid State lonics 129 (1), 111 (2000).

Wyslouzil, B. E., Wilemski, G., Strey, R., "Aerosol SANS: A New Method to Probe the Structure of Nanodroplets," in Nucleation an Atmospheric Aerosols, edited by B. Hale, M. Kulmala (American Institute of Physics, 2000), p. 724.

Xu, G., Aeppli, G., Bisher, M. E., Broholm, C., DiTusa, J. F., Frost, C. D., Ito, T., Oka, K., Takagi, H., Treacy, M. M. J., "Holes in a Quantum Spin Liquid," Science 289 (5478), 419 (2000).

Xu, G., Broholm, C., Reich, D. H., Adams, M. A., "Triplet Waves in Quantum Spin Liquid," Phys. Rev. Lett., in press.

Yamaura, K., Huang, Q., Erwin, R.W., Lynn, J. W., Cava, R. J., "A Study of Structure Electric and Magnetic Properties of the New Layered Cobalt Oxide $\mathrm{Sr}_{2} \mathrm{Y}_{1-x} \mathrm{Ca}_{x} \mathrm{Co}_{2} \mathrm{O}_{7}$," Low Temp. Phys. 117 (3), 849 (1999).

Yamaura, K., Huang, Q., Lynn, J. W., Erwin, R. W., Cava, R. J., "Synthesis, Crystal Structure and Magnetic Order of the Layered Iron Oxycarbonate $\mathrm{Sr}_{4} \mathrm{Fe}_{2} \mathrm{O}_{6} \mathrm{CO}_{3}$, J. Solid State Chem. 152 (2), 374 (2000).

Yamaura, K., Huang, Q., Takayama-Muromachi, E., "Crystal Structure and Magnetism of the Linear-Chain Copper Oxides $\mathrm{Sr}_{5} \mathrm{~Pb}_{3-\mathrm{x}} \mathrm{Bi}_{x} \mathrm{CuO}_{12}$ " Phys. Rev. B, in press.

Yang, L., Alexandridis, P., " Polyoxyalkylene Block Copolymers in Formamide-Water Mixed Solvents: Micelle Formation and Structure Studied by Small-Angle Neutron Scattering" Langmuir 16 (11), 4819 (2000).

Yang, L., Harroun, T. A., Heller, W. T., Weiss, T. M., Huang, H., W., "Supramolecular Structures of Peptide Assemblies in Membranes by Neutron Off-Plane Scattering: Method of Analysis," Biophys. J. 77 (5), 2648 (1999).

Yang, L., Slawecki, T. M., Alexandridis, P., "Shear Induced Orientation and Structural Transitions in Micellar Cubic Crystals Formed by Solvated Block Copolymers: A SANS Investigation," Macromol., in press.
Yildirim, T., "Structure and Dynamics from Combined Neutron Scattering and FirstPrinciples Studies," Chem. Phys., in press.

Yildirim, T., Ciraci, S., Kilic, C., Buldum, A., "First-Principles Investigation of Structural and Electronic Properties of Solid State Cubane and its Doped Derivatives" Phys. Rev. B 62 (11), 7625 (2000).

Yildirim, T., Gehring, P. M., Neumann, D. A., "The Dynamics of Solid Cubane," Neutron News, in press.

Yildirim, T., Gulseren, O., Ciraci, S., Kilic, C., "Pressure-Induced Interlinking of Carbon Nanotubes," Phys. Rev. B, in press.

Yildirim, T., Neumann, D. A., Trevino, S. F., Fischer, J. E., "Neutron Scattering Study of $\mathrm{Na}_{2} \mathrm{C}_{60}$ in Pressure-Temperature Plane," Phys. Rev. B 60 (15), 10707 (1999).

Yildirim, T., Zhou, O., Fischer, J. E., "Intercalation Compounds of Fullerenes I: Synthesis, Characterization, and Solid State Properties," in The Physics of Fullerene Based and Fullerene Related Materials, edited by W. Andreoni (Kluwer Academic Publishers, Dordrecht, The Netherlands, 2000), p. 66

Yildirim, T., Zhou, O., Fischer, J. E. "Intercalation Compounds of Fullerenes II: Structure and Superconductivity of Alkali Metal Fullerides," in The Physics of Fullerene Based and Fullerene Related Materials, edited by W. Andreoni (Kluwer Academic Publishers, Dordrecht, The Netherlands, 2000), p. I33.

Yildirim, T., Zhou, O., Fischer, J. E., "Intercalation Compounds of Fullerenes III: Other Fullerides and Intercalated Nanotubes," in The Physics of Fullerene Based and Fullerene Related Materials, edited by W. Andreoni (Kluwer Academic Publishers, Dordrecht, The Netherlands, 2000), p.289.

Yim, H., Kent, M., Matheson, A., Ivkov, R., Satija, S., Majewski, J., Smith, G. S., "Adsorption of Polystyrene Sulfonate to the Air Surface of Water by Neutron Reflectivity," Macromol. 33 (16), 6126 (2000).

Yim, H., Kent, M., McNamara, W. F., Ivkov, R., Satija, S., Majewski, J., "Structure within Thin Epoxy Films Revealed by Solvent Swelling: A Neutron Reflectivity Study," Macromol. 32 (23), 7923 (1999).

Zaliznyak, I. A., Broholm, C., Kibune, M., Nohara, M., Takagi, H., "Anisotropic Spin Freezing in the $\mathrm{S}=1 / 2$ Zigzag Chain Compound $\mathrm{SrCuO}_{2}$," Phys. Rev. Lett. 83 (25) 5370 (1999).

Zaliznyak, I. A., Hill, J. P., Tranquada, J. M., Erwin, R., Moritomo, Y., "Independent Freezing of Charge and Spin Dynamics in $\mathrm{La}_{1.5} \mathrm{Sr}_{0.5} \mathrm{CoO}_{4}$," Phys. Rev. Lett., in press.

Zaliznyak, I., Broholm, C., Kibune, M., Takagi, H., "Spin Freezing in the Cuprate $S=1 / 2$ Antiferromagnetic Chain Compound $\mathrm{SrCuO}_{2}$," Phys. Rev. Lett., in press.

Zamlynny, V., Burgess, 1., Szymanski, G., Lipkowski, J., Majewski, J., Smith, G., Satija, S., Ivkov, R., "Electrochemical and Neutron Reflectivity Studies of Spontaneously Formed Amphiphilic Surfactant Bilayers at the Gold-Solution," J. Electrochem. Soc., in press.

Zeisler, R., "Investigations by INAA for the Development of Natural Matrix Standard Reference Materials (R)(SRMs) Suitable for Small Sample Analysis," J. Radioanal. Nucl. Ch. 245 (1), 81 (2000).

Zeisler, R., "Maintaining Accuracy in Gamma-Ray Spectrometry at High Count Rates," J. Radioanal. Nucl. Ch. 244 (3), 507 (2000).

Zeisler, R., Greenberg, R. R., "Determinations of Subnanomole Elemental Levels by NAA and Their Possible Impact on Human Health Related lssues," Biol. Trace Elem. Res. 71 (2), 283 (1999).

Zeisler, R., Jenks, P., "Chapter 7-Proper Usage of Reference Materials," in Reference Materials for Chemical Analysis - Cerification, Availability, and Proper Usage, edited by M. Stoeppller, W. R. Wolf, P. Jenks (John Wiley and Sons, Inc., New York, NY), in press.

Zeisler, R., Lamaze, G. P., Chen-Mayer, H. H., "Coincidence and Anti-Coincidence Measurements in Prompt Gamma Neutron Activation Analysis with Pulsed Cold Neutron Beams," J. Radioanal. Nucl. Ch., in press.

Zhang, Y., Douglas, J. F., Ermi, B. D., Amis, E. J., "Influence of Counterion Valency on the Scattering Properties of Highly Charged Polyelectrolyte Solutions," J. Chem. Phys., in press. 
High resolution powder diffractometer (BT-1)

J. K. Stalick, (301) 975-6223, judy.stalick@nist.gov

B. H. Toby, (301) 975-4297, brian.toby@nist.gov

DARTS, Residual stress and texture diffractometer (BT-8)

H. J. Prask, (301) 975-6226, hank@nist.gov

P. C. Brand, (301) 975-5072, pbrand@nist.gov

$30 \mathrm{~m}$ SANS instrument (NG-7)

C. J. Glinka, (301) 975-6242, cglinka@nist.gov

J. B. Barker, (301) 975-6732, barker@nist.gov

J. Schulz, (301) 975-6469, jamie.schulz@nist.gov

30 m SANS instrument (NG-3) (NIST/NSF-CHRNS)

B. Hammouda, (301) 975-3961, hammouda@nist.gov

S. R. Kline, (301) 975-6243, steven.kline@nist.gov

S. Krueger, (301) 975-6734, krueger@nist.gov

$8 \mathrm{~m}$ SANS instrument (NG-1)

A. Karim, (301) 975-6588, karim@nist.gov

USANS, Perfect crystal SANS (BT-5) (NIST/NSF-CHRNS)

J. B. Barker, (301) 975-6732, barker@nist.gov

Cold neutron reflectometer-vertical sample-polarized beam option (NG-1)

C. F. Majkrzak, (301) 975-5251, cmajkrzak@nist.gov J. A. Dura, (301) 975-6251, jdura@nist.gov

Cold neutron reflectometer-horizontal sample (NG-7)

S. K. Satija, (301) 975-5250, satija@nist.gov

R. Ivkov, (301) 975-4662, rivkov@nist.gov

Triple-axis polarized-beam spectrometer (BT-2) J. W. Lynn, (301) 975-6246, jeff.Iynn@nist.gov

Triple-axis fixed incident energy spectrometer (BT-7) J. W. Lynn, (301) 975-6246, jeff.lynn@nist.gov

Triple-axis spectrometer (BT-9)

R.W. Erwin, (301) 975-6245, rerwin@nist.gov

P. M. Gehring, (301) 975-3946, pgehring@nist.gov

SPINS, Spin-polarized triple-axis spectrometer (NG-5) (NIST/NSF-CHRNS)

P. M. Gehring, (301) 975-3946, pgehring@nist.gov

S.-H. Lee, (301) 975-4257, seung-hun.lee@nist.gov
FANS, Filter-analyzer neutron spectrometer (BT-4)

T. J. Udovic, (301) 975-6241, udovic@nist.gov

P. Papanek, (301) 975-5049, pete@jazz.ncnr.nist.gov

D. A. Neumann, (301) 975-5252, dan@nist.gov

FCS, Fermi-chopper time-of-flight spectrometer (NG-6) C. M. Brown, (301) 975-5134, craig.brown@nist.gov

T. J. Udovic, (301) 975-6241, udovic@nist.gov

DCS, Disk-chopper time-of-flight spectrometer (NG-4)

J. R. D. Copley, (301) 975-5133, jcopley@nist.gov

J. C. Cook, (301) 975-6403, jeremy.cook@nist.gov

High-flux backscattering spectrometer (NG-2)

R. M. Dimeo, (301) 975-8135, robert.dimeo@nist.gov

Z. Chowdhuri, (301) 975-4404, zema@nist.gov

NSE, Neutron spin echo spectrometer (NG-5)

N. S. Rosov, (301) 975-5254, nrosov@nist.gov

Prompt-gamma neutron activation analysis (NG-7)

R. M. Lindstrom, (301) 975-6281, dick.lindstrom@nist.gov

R. L. Paul, (301) 975-6287, rpaul@nist.gov

Other activation analysis facilities

R. R. Greenberg, (301) 975-6285, rgreenberg@nist.gov

Cold neutron depth profiling (NG-0)

G. Lamaze, (301) 975-6202, lamaze@nist.gov

Instrument development station (NG-0)

D. F. R. Mildner, (301) 975-6366, mildner@nist.gov

H. H. Chen-Mayer, (301) 975-3782, chenmayer@nist.gov

Neutron interferometer (NG-7)

M. Arif, (301) 975-6303, muhammad.arif@nist.gov

Fundamental neutron physics station (NG-6)

M. S. Dewey, (301) 975-4843, mdewey@nist.gov

Theory and modelling

N. F. Berk, (301) 975-6224, nfb@nist.gov

T. Yildirim, (301) 975-6228, taner@nist.gov

Sample environment

D. C. Dender, (301) 975-6225, dender@nist.gov

L. J. Santodonato, (301) 975-6657, Isantodonato@nist.gov 


\section{NIST CENTER \\ FOR NEUTRON \\ RESEARCH}

For copies of or information on this report, contact:

Ronald L. Cappelletti

(310) $975-6221$

ron.cappelletti@nist.gov

For additional information on the facility,

contact:

\section{J. Michael Rowe \\ (301) $975-6210$ \\ mike.rowe@nist.gov \\ John J. Rush \\ (301) 975-6231 \\ john.rush@nist.gov}

To obtain guidelines for preparing proposals to conduct research at the facility, contact:

\section{William Kamitakahara}

(301) 975-6878

william.kamitakahara@nist.gov

Location of all contacts:

NIST Center for Neutron Research Building 235, Room E151, Mail Stop 8562

National Institute of Standards and Technology Gaithersburg, MD 20899-8562

Details are also available on the NCNR Web site:

www.nenr.nist.gov. 
